\title{
Following Advice Because it's Been Paid For: Age, the Sunk-Cost Fallacy, and Loss Aversion
}

\author{
Leo Schlosnagle \\ West Virginia University
}

Follow this and additional works at: https://researchrepository.wvu.edu/etd

\section{Recommended Citation}

Schlosnagle, Leo, "Following Advice Because it's Been Paid For: Age, the Sunk-Cost Fallacy, and Loss Aversion" (2011). Graduate Theses, Dissertations, and Problem Reports. 4783.

https://researchrepository.wvu.edu/etd/4783

This Dissertation is protected by copyright and/or related rights. It has been brought to you by the The Research Repository @ WVU with permission from the rights-holder(s). You are free to use this Dissertation in any way that is permitted by the copyright and related rights legislation that applies to your use. For other uses you must obtain permission from the rights-holder(s) directly, unless additional rights are indicated by a Creative Commons license in the record and/ or on the work itself. This Dissertation has been accepted for inclusion in WVU Graduate Theses, Dissertations, and Problem Reports collection by an authorized administrator of The Research Repository @ WVU.

For more information, please contact researchrepository@mail.wvu.edu. 
Following Advice Because it's Been Paid For: Age, the Sunk-Cost Fallacy, and Loss Aversion

Leo Schlosnagle, M. S.

Dissertation submitted to the

College of Arts and Sciences at

West Virginia University

in partial fulfillment of the requirements

for the degree of

Doctor of Philosophy

in

Psychology

JoNell Strough, Ph.D., Chair

Julie Hicks Patrick, Ph.D.

Michael Perone, Ph.D.

Elizabeth Kyonka, Ph.D.

William Trumbull, Ph.D.

Department of Psychology

Morgantown, West Virginia

2011

Keywords: sunk-cost fallacy, loss aversion, monetary investments, age differences, delay discounting 


\begin{abstract}
Following Advice Because it's Been Paid For: Age, the Sunk-Cost Fallacy, and Loss Aversion Leo Schlosnagle

The sunk-cost fallacy occurs when a person invests more of a resource because they have made an irrecoverable initial investment (i.e., a sunk cost) compared to scenarios in which they did not make an initial investment, or made a smaller initial investment. The normatively correct decision is to invest exactly the same amount regardless of whether or not an initial investment has been made. An aversion to "wasting" or "losing" the initial investment has been cited as a potential reason for why people commit the sunk-cost fallacy (Arkes \& Blumer, 1985; Soman, 2004). Additionally, younger adults commit the sunk-cost fallacy more frequently, and make normatively correct decisions less frequently, than older adults when hypothetical sunk costs are at stake (Strough, Mehta, McFall, \& Schuller, 2008). In the present study, younger ( $n=50, M$ age $=20.44,68 \%$ women $)$ and older $(n=50, M$ age $=69.74,56 \%$ women $)$ adults responded to a Calorie Estimation Task measure of the sunk-cost fallacy involving investments and potential returns of real money, as well as a self-report measure involving hypothetical investments of time and money. Younger adults made fewer initial investments of money on the Calorie Estimation Task than older adults. Younger adults demonstrated the sunk-cost fallacy more frequently, overinvested more after demonstrating the fallacy, and made the normatively correct decision less frequently than older adults on the hypothetical self-report measure of the sunk-cost fallacy. Younger adults indicated that they were more averse to hypothetical monetary losses on a Tradeoff Loss Aversion Task and more averse to hypothetical monetary losses on a Delay Discounting Loss Aversion Task. An inverse (although non-significant) relation between Tradeoff Loss Aversion Task Scores and the Spendthrift Tightwad Scale Scores (STS; Rick, Cryder, \& Loewenstein, 2008) provided preliminary evidence for convergent validity of the Tradeoff Loss Aversion Task. A positive (although non-significant) relation between Delay Discounting Loss Aversion Scores and STS Scores suggested that the Delay Discounting Loss Aversion Task lacked convergent validity as a measure of loss aversion. Neither measure of loss aversion mediated the inverse relation between age and demonstration of the sunk-cost fallacy on the self-report measure of the sunk-cost fallacy. Participants' ability to estimate calories accurately, their confidence in their ability to estimate calories accurately, and their difficulty paying bills did not relate to rates of advice purchasing. Older adults did not demonstrate the sunk-cost fallacy on the Calorie Estimation Task by following more expensive advice more closely than less expensive advice. Exploratory analyses, implications and limitations of the present study, and potential directions for future research are discussed.
\end{abstract}




\section{DEDICATION}

I would like to dedicate this dissertation to my parents, Steve and Eileen — both of whom made sacrifices to ensure that I received an extraordinary education. Moreover, I would like to thank you for the unwavering love and support that you've both provided over the years-I love you both. Additionally, I would like to dedicate this dissertation to my friend Jim McNeil, who passed away earlier this year. Jim, you were an exemplary mentor, and we could all better ourselves by emulating your genuine compassion, modesty and moral fidelity. You were a true hero, and you are missed dearly. 


\section{ACKNOWLEDGEMENTS}

I would like to acknowledge and thank my Advisor, Dr. JoNell Strough for her assistance with, and feedback regarding, this dissertation. I would also like to acknowledge and thank my dissertation committee—Dr. JoNell Strough (Chair), Dr. Julie Hicks Patrick, Dr. Michael Perone, Dr. Elizabeth Kyonka, and Dr. William Trumbull—-for their valuable insight and feedback regarding this dissertation. Additionally, I would like to acknowledge and thank all of my mentors, teachers, friends and family members who have provided support and guidance over the years - including (but certainly not limited to) Dr. David Finkelman, Dr. Elizabeth N. Williams, Dr. Sherry Shuman, Dr. David Jarmolowicz, Allison Tetreault, Collen DuBose, Wade Wiley, Kevin Fogle, Zachary Levine, Eastman Lewis, Zachary Bankert, Victor Telisa, Rebecca Nelson, Caitlin Sussman, Brooks DuBose, Jack Dubose, Caroline DuBose, Barry Gregory, Nakia Beasley, Michael Sweeney, John Van Oudenaren, Lydia Hastings and Jon Ortega. Additionally, I would like to thank my undergraduate research assistants-Paul Michael Weisberg, Anna DeFrancis, Shannon Szabo, Kristen Bock, Clara Simmons, and Samantha Lauri-their assistance with this project was invaluable. I would also like to acknowledge the American Psychological Association, which awarded this project the APA Dissertation Research Award in 2010 and provided funding for this research. Lastly, I would also like to acknowledge the WVU Department of Psychology which provided funding for this project, and the Center for Excellence in Disabilities, which provided computer hardware and software that was used to complete this project. 


\section{TABLE OF CONTENTS}

TITLE PAGE .

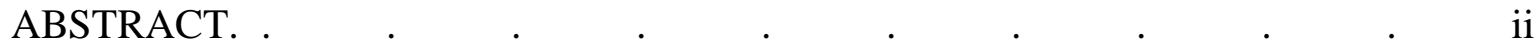

DEDICATION.

ACKNOWLEDGEMENTS . $. \quad . \quad . \quad . \quad . \quad . \quad . \quad$ iv

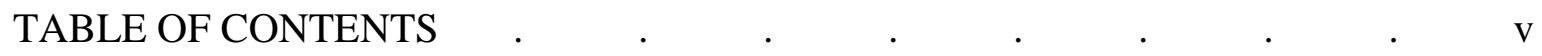

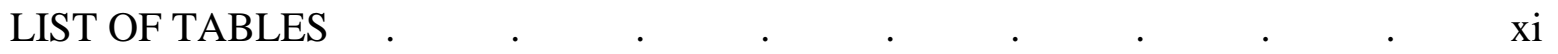

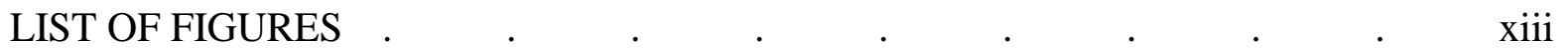

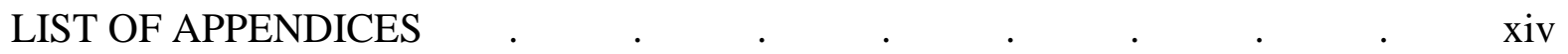

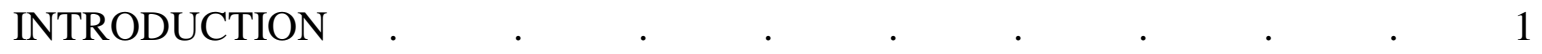

The Sunk-Cost Fallacy: Parameters and Definitions . $\quad$. $\quad$. $\quad 2$

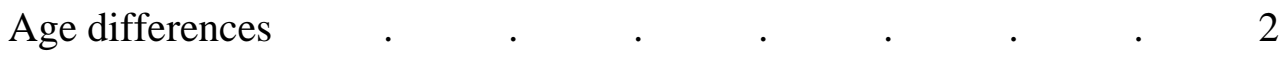

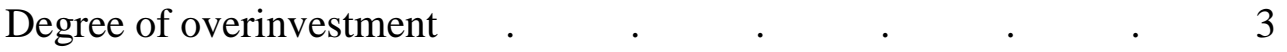

Normatively correct decisions $\quad . \quad$. $\quad . \quad$. $\quad$. 3

$\begin{array}{lllllllll}\text { Absolute vs. proportional investments } & \text {. } & \text {. } & \text {. } & & & 6\end{array}$

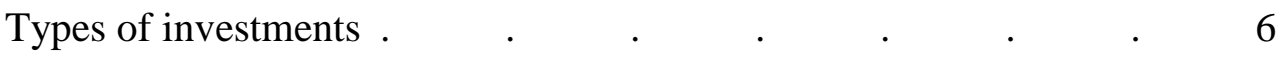

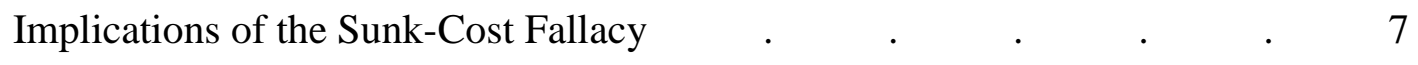


Potential Explanations of Investment Behaviors and the Sunk-Cost Fallacy.

Explanations of Age Differences in the Sunk-Cost Fallacy . $\quad$. $\quad 12$

Loss Aversion, Delay discounting of Gains and Losses, and Monetary Tradeoffs 15

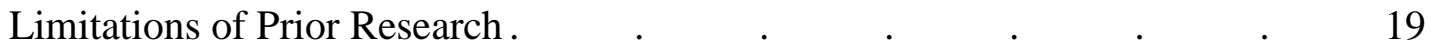

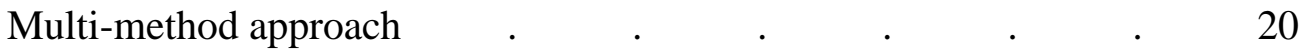

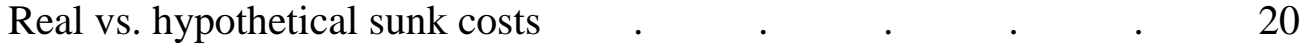

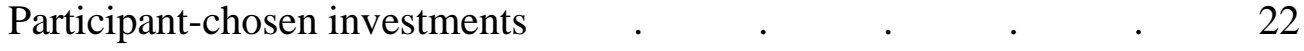

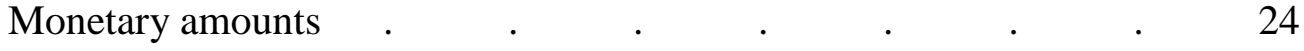

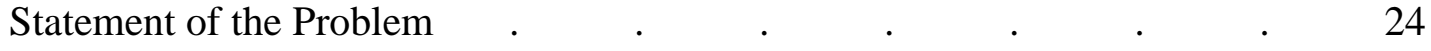

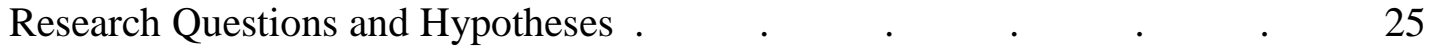

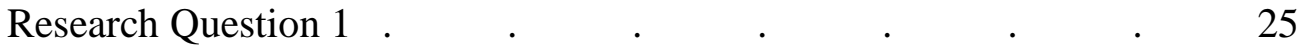

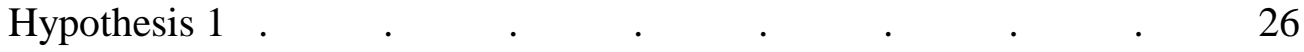

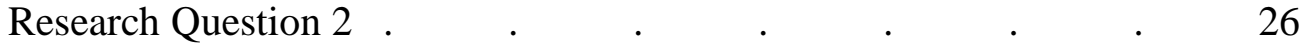

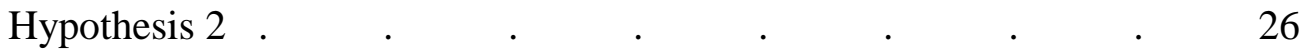

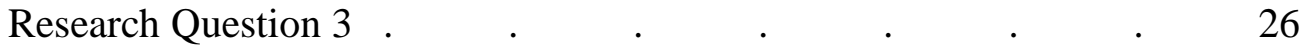

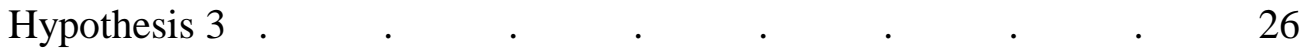

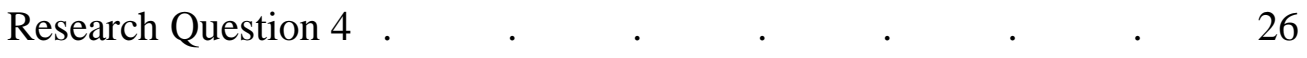




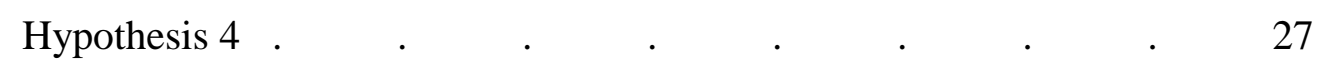

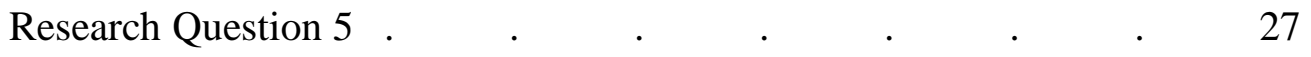

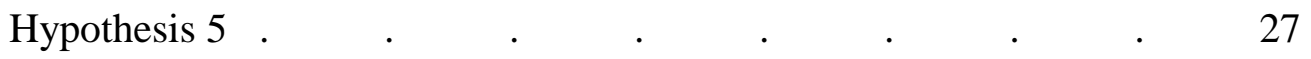

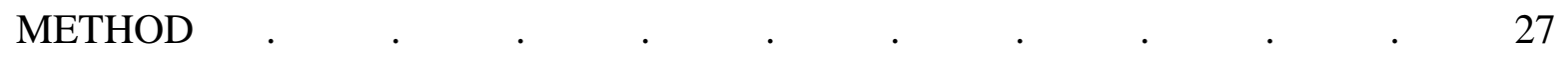

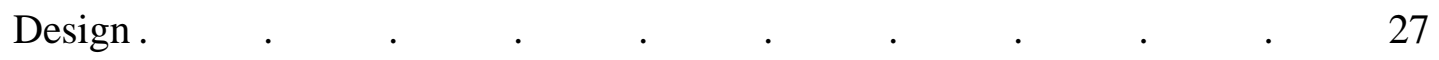

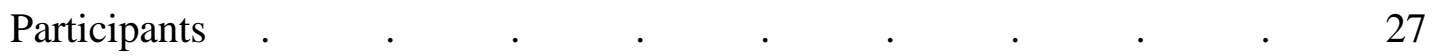

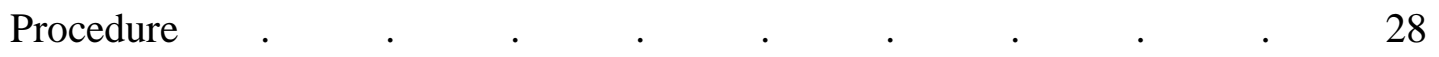

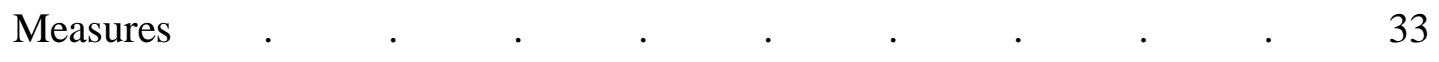

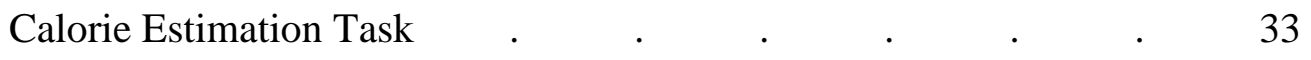

Calorie Estimation Task pilot studies . $\quad$. $\quad$. $\quad$. 35

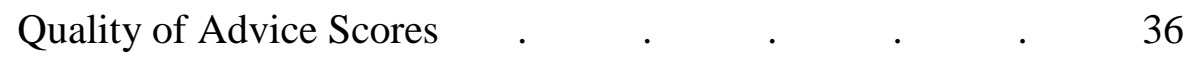

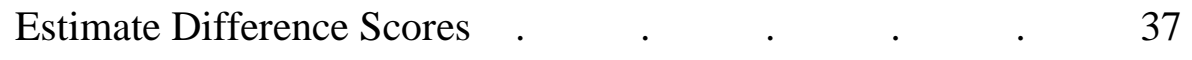

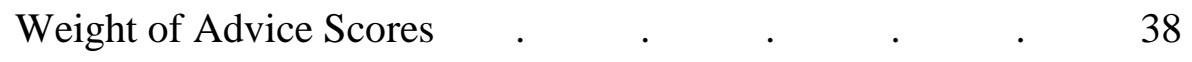

Calorie Estimate Sunk-Cost Fallacy Scores . $\quad$ • $\quad$. 48

Advice Adherence Scores $\quad . \quad$. $\quad$. $\quad$. $\quad$. $\quad$. 40

Advice Adherence Sunk-Cost Fallacy Scores. $\quad$ 43

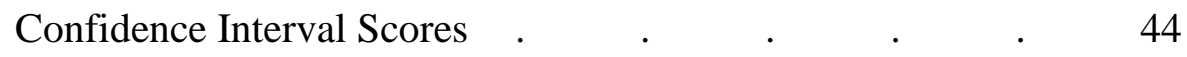




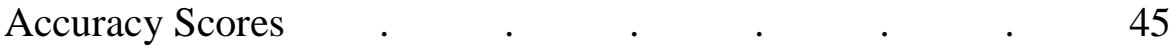

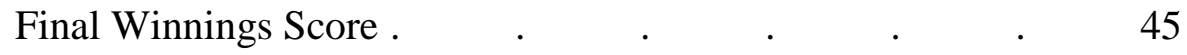

Loss Aversion: Delay Discounting Task $\quad . \quad$. $\quad$. $\quad$. $\quad 45$

Delay Discounting Loss Aversion Score. 47

Reversed AUC Gains and Losses Scores _ . $\quad$. $\quad 4 \quad 49$

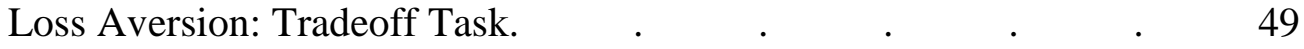

Tradeoff Loss Aversion Task Score . $\quad$. $\quad$. $\quad$. 51

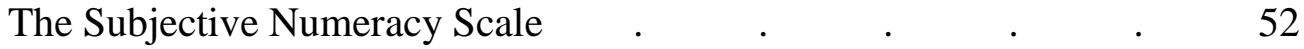

The weight control subscale of the Food Choice Questionnaire . $\quad$. 52

The Health Habits Questionnaire . $\quad$. $\quad$. $\quad$. $\quad$. 53

The Sunk Cost Vignette Questionnaire . $\quad . \quad$. $\quad$. $\quad$. 53

Sunk-Cost Fallacy Vignette Scores . $\quad$. $\quad$. $\quad$. 54

Sunk-Cost Fallacy Vignette Overinvestment Scores . $\quad$. 55

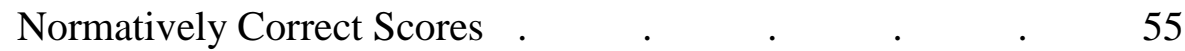

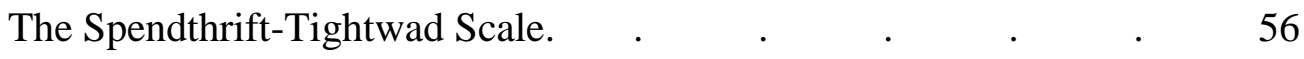

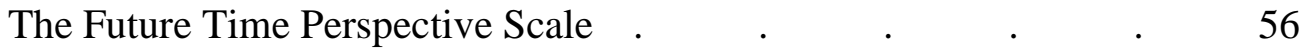

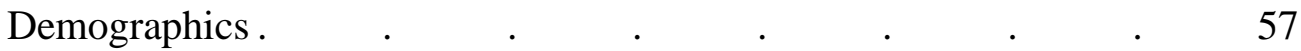




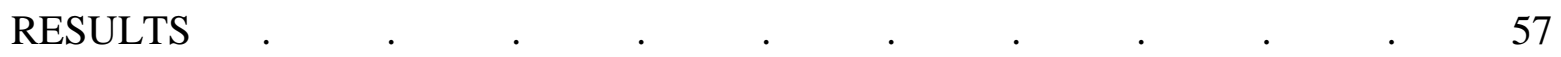

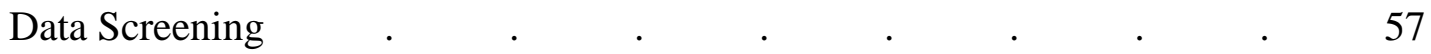

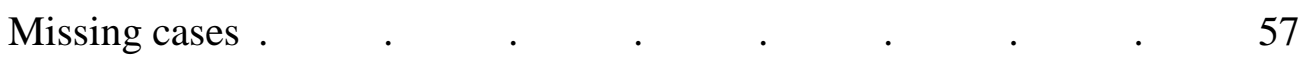

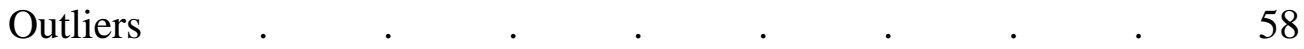

Nonsystematic delay discounting data. $\quad . \quad$. $\quad . \quad$. $\quad 58$

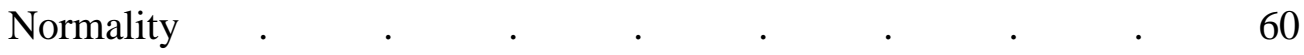

Assumption of homogeneity of variances. . $\quad . \quad$. $\quad . \quad 60$

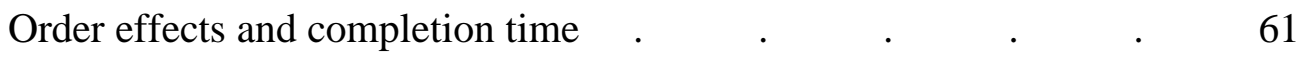

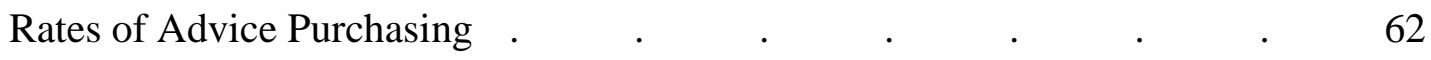

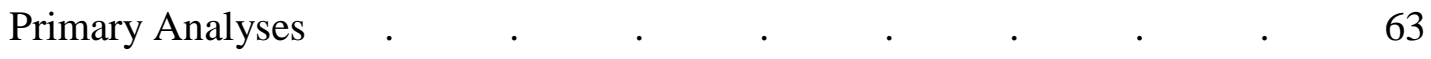

RQs 1, 2, \& 3: Age differences in the sunk-cost fallacy, normatively correct decisions, overinvestment, and loss aversion

RQ 4: Calorie advice purchasing and confidence in calorie estimates

RQ 5: Loss aversion as a mediator of the relation between age and the sunk-cost fallacy . $\quad . \quad$. $\quad . \quad$. $\quad 66$

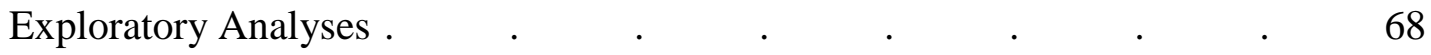

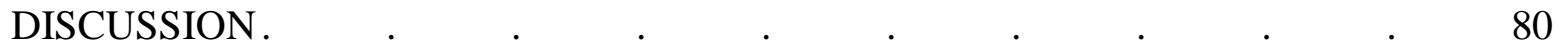

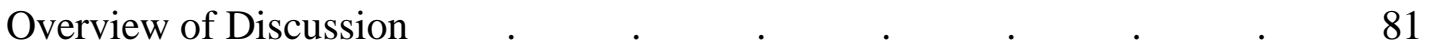


Rates of Advice Purchasing and Willingness to Pay . $\quad$. $\quad$. $\quad$. 82

The Sunk-Cost Fallacy, Normatively Correct Decisions, and Overinvestment $\quad 89$

Loss Aversion as a Mediator of Age Differences in the Sunk-Cost Fallacy . $\quad 91$

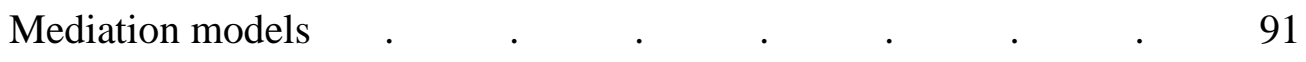

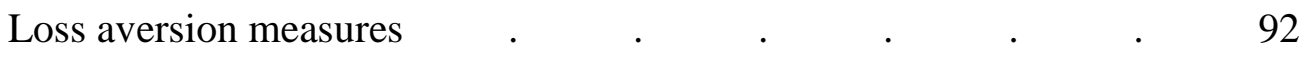

Delay Discounting, Loss Aversion and Future Time Perspective 95

Implications, Limitations, and Future Directions $\quad . \quad$. $\quad . \quad$. 100

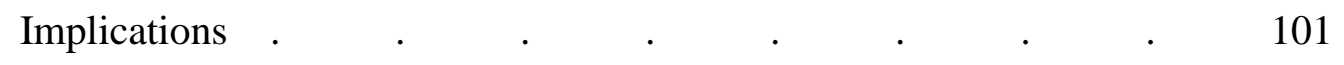

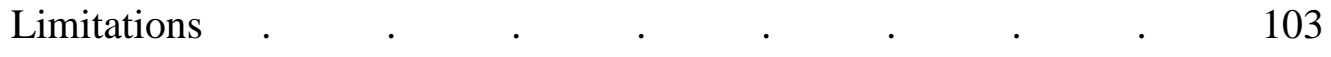

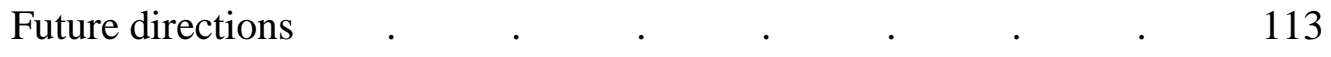

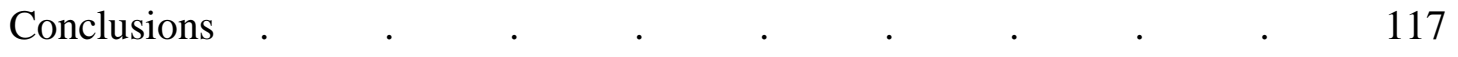

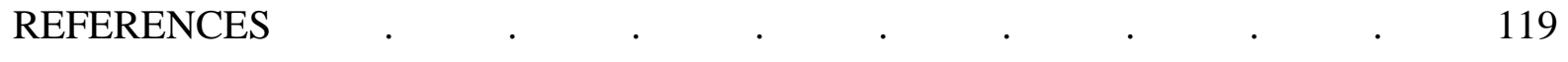

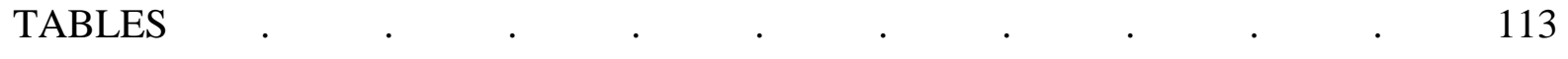

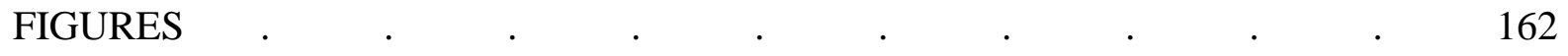

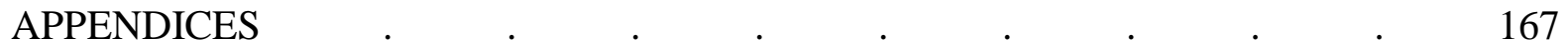




\section{LIST OF TABLES}

Table 1: Experimental Design of Calorie Estimation Task _ $\quad . \quad$. $\quad$. 133

Table 2: Demographic Characteristics Separated by Age Group . 134

Table 3: Descriptive Statistics for Primary Variables. $\quad . \quad$. $\quad . \quad$. $\quad 141$

Table 4: Descriptive Statistics for Primary Variables, Separated by Age Group 143

Table 5: Pearson Correlations for Primary Variables (Entire Sample; Younger

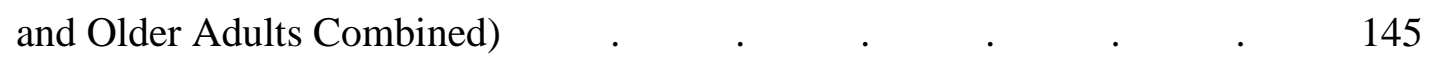

Table 6: Pearson Correlations for Primary Variables by Age Group $\quad$ 150

Table 7: Pearson Correlations: Weight Control Sub-Scale of the Food Choice Questionnaire Score, Health Habits Questionnaire Score, Mean Pre-Advice Confidence Interval Score, and Pre-Advice Calorie Estimate Accuracy Score; for the Entire Sample (Younger and

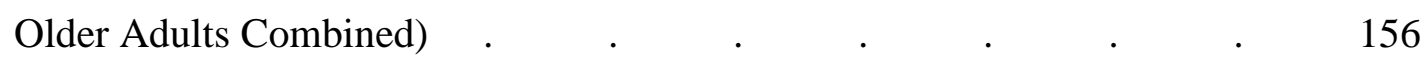

Table 8: Pearson Correlations: Weight Control Sub-Scale of the Food Choice Questionnaire Score, Health Habits Questionnaire Score, Mean Pre-Advice Confidence Interval Score, and Pre-Advice Calorie Estimate Accuracy Score for Younger and for Older Adults

Table 9: Pearson Correlations: the Subjective Numeracy Scale, Reversed AUC Gains Scores, Reversed AUC Losses Scores and FTP Scores for the Entire Sample (Younger and Older Adults Combined) . 
Table 10: Pearson Correlations: the Subjective Numeracy Scale, Reversed AUC Gains Scores, Reversed AUC Losses Scores, and FTP Scores for

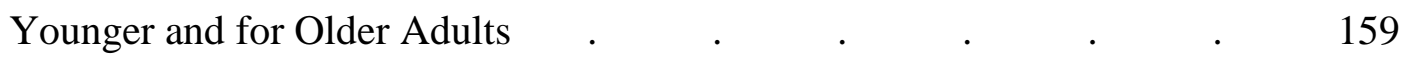

Table 11: Delay Discounting Loss Aversion Score as a Mediator of the Relation Between Age Group and Sunk-Cost Fallacy Vignette Score $\quad$. $\quad$. 160

Table 12: Tradeoff Loss Aversion Task Score as a Mediator of the Relation Between Age Group and Sunk-Cost Fallacy Vignette Score. . . . 161 


\section{LIST OF FIGURES}

Figure 1: Age differences in Sunk-Cost Fallacy Vignette, Sunk-Cost Fallacy Vignette Overinvestment, and Normatively Correct Scores. $\quad$. $\quad$. 162

Figure 2: Age differences in Delay Discounting Loss Aversion Scores 163

Figure 3: Age differences in Tradeoff Loss Aversion Task Scores . $\quad$ 164

Figure 4: Age differences in Reversed AUC Gains and Losses Scores 165

Figure 5: Example illustration of graphed normalized subjective values used to calculate AUC Gains and Losses Scores and the Delay Discounting

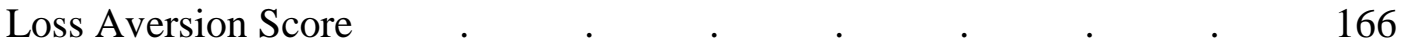




\section{LIST OF APPENDICES}

Appendix A: Calorie Estimation Task and Instructions $\quad . \quad$. $\quad . \quad$. 170

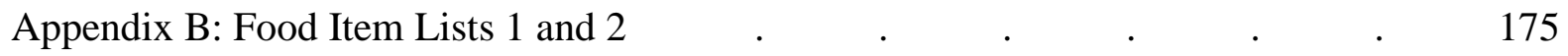

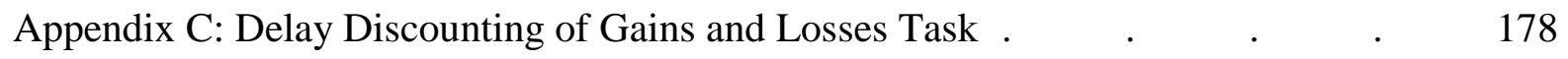

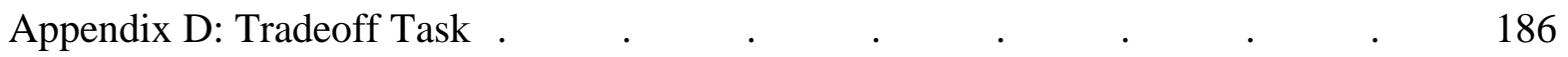

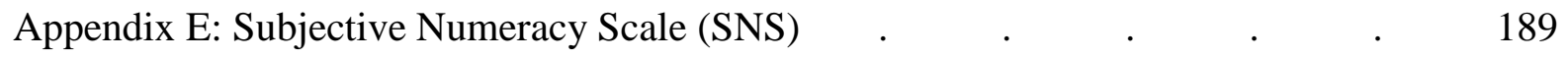

Appendix F: Weight Control Subscale of Food Choice Questionnaire (FCQ) and the Health Habits Questionnaire (HHQ) . $\quad$. $\quad$. $\quad$. 191

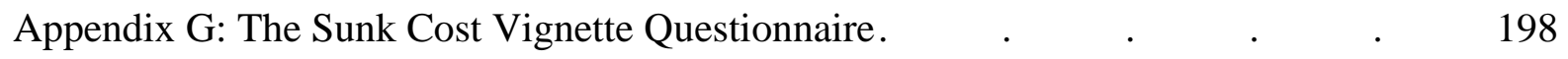

Appendix H: Spendthrift-Tightwad Scale (STS) . $\quad . \quad$. $\quad . \quad$. $\quad 206$

Appendix I: Future Time Perspective (FTP) Scale . $\quad . \quad$. $\quad$. $\quad$. 209

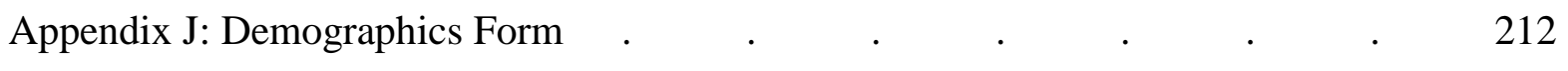

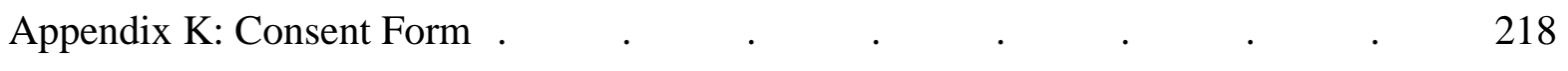




\section{Introduction}

The sunk-cost fallacy is a decision-making bias that occurs when a person invests more of a particular resource because a prior investment has been made (i.e., costs have been "sunk"), compared to scenarios in which no prior investment has been made (Arkes \& Blumer, 1985), or scenarios in which the prior investment was small (e.g., Strough, Mehta, McFall, \& Schuller, 2008). The sunk-cost fallacy is irrational because the decision of whether to continue investment should be based on expected future costs and returns, not on irretrievable prior investments (Arkes \& Blumer). Most prior studies of the sunk-cost fallacy have employed self-report measures involving hypothetical investments of money (e.g., Arkes, 1996), often in amounts greater than those encountered in everyday life, and which were not actively chosen by the participant (e.g. Arkes \& Hutzel, 2000; Bornstein \& Chapman, 1995; Bruine de Bruin, Parker \& Fischoff, 2007; Coleman, 2010; Garland \& Newport, 1991; Keil, Truex III, \& Mixon, 1995; Larrick, Nisbett, \& Morgan, 1993; Stanovich \& West, 1999). This study is one of the first to assess whether people choose to make investments and demonstrate the sunk-cost fallacy when the investments and potential returns are real monetary amounts typical of those encountered in everyday life. This study is unique in that it compares traditional self-report measure of the sunkcost fallacy to a measure involving real monetary investments and potential returns. Because a decision to make an initial investment is a pre-requisite for the sunk-cost fallacy, and because people may be more willing make hypothetical payments than real payments (e.g., Blumenschein

et al., 1998; Botelho \& Pinto, 2001), the assessment of peoples' willingness to pay real money in the present study addresses an essential component of the sunk-cost fallacy. Moreover, this study assesses whether monetary loss aversion accounts for age differences in the sunk-cost fallacy, which have been observed in prior studies (e.g., Strough, Karns, \& Schlosnagle, 2011a; Strough, Schlosnagle, \& DiDonato, 2011b). 
The introduction that follows begins with an overview of previously-studied parameters of the sunk-cost fallacy, as well as definitions of related phenomena. Then, potential implications and explanations of the sunk-cost fallacy are considered, followed by a description of some of the limitations of prior studies of the sunk-cost fallacy.

\section{The Sunk-Cost Fallacy: Parameters and Definitions}

Age differences. Studies that use hypothetical sunk cost scenarios show that older adults exhibit a greater resistance to sunk costs than do younger adults. Specifically, older adults ignore prior investments more frequently than younger adults when deciding whether or not to continue investing in a course of action (Bruine de Bruin et al., 2007). Similarly, older adults demonstrate the sunk-cost fallacy less frequently than younger adults when hypothetical sunk costs are at stake (Strough et al., 2011a). This stands in contrast to some other aspects of decision-making abilities, which older adults have more difficulty with when compared to younger adults (Bruine de Bruin et al.; Denburg, Tranel, \& Bechara, 2005; see Peters, Hess, Västfjäll, \& Auman, 2007 for a review).

Older adults comprise a considerable proportion of the population of the United States; the number of people over the age of 60 living in the United States has increased considerably in recent years, and is projected to continue increasing for several more decades (Carstensen \& Hartel, 2006; Meyer, 2001). Moreover, older individuals must often decide how to invest resources in a variety of domains such as personal healthcare, finances, and retirement plans (Bassett, Fleming, \& Rodrigues, 1998; Chen \& Sun, 2003; Denburg et al, 2005; Holden \& VanDerhei, 2001). Thus, it is increasingly relevant to understand the unique decision-making abilities and deficits that older adults experience, such as their decreased likelihood of 
demonstrating the sunk-cost fallacy compared to younger adults (Strough et al., 2011a). Several studies have investigated age differences in how younger and older adults respond to hypothetical sunk costs (e.g., Bruine de Bruin et al., 2007; Strough et al., 2008; 2011b), and others have studied the effects of real, rather than hypothetical sunk costs with college undergraduates (e.g., Navarro \& Fantino, 2005). However, this study compares how younger and older adults perform on a hypothetical self-report measure of the sunk-cost fallacy to how they perform on a measure involving real monetary investments and potential returns.

Degree of overinvestment. This study uses the term "degree of overinvestment" to refer to how many additional investments a person commits after demonstrating the sunk-cost fallacy. Some have described the same phenomena by referring to it as "escalation of commitment". However, the phrase "escalation of commitment" has also been used to describe several other similar phenomena. For instance, some have referred to instances of continued engagement with a failing course of action as "escalation of commitment" (Brockner, 1992; Staw, 1981). Others have referred to "escalation of commitment" as being the same as the sunk-cost fallacy (Beeler \& Hunton, 1997), or instances in which an initial investment increases the likelihood of the investment of additional resources (Garland, 1990; Heath, 1995). Thus, only the terms "overinvestment" and "degree of overinvestment" are used in this study, and they describe instances in which a person demonstrates the sunk-cost fallacy by committing more than an optimal amount of resources following an initial investment, and then goes on to invest further resources. This study compares younger and older adults' degree of overinvestment.

Normatively correct decisions. When considering what decision to make after committing an investment (i.e., a sunk cost), people may either invest more, less, or the same amount of resources compared to a similar scenario where no prior investment had been made. 
When a person decides to invest exactly the same amount of resources in a scenario where a sunk cost has been committed compared to one without a sunk cost, they are said to be making the normatively correct decision (i.e., their decision is in no way influenced by the presence or absence of a sunk cost; Klaczynski, 2001). This definition of normative decision making is based on models that define "normative" as optimal responses that adhere to principles of logic and utility theory (i.e., that the way in which identical alternatives are presented should not affect peoples' decisions regarding those alternatives). Put differently, under this definition, "normative" decision making is decision making that is in no way influenced by aspects of the decision making context that do not affect the utility of the final decision (Kahneman \& Tversky, 1979). For instance, the presence or absence of an investment of time in a romantic relationship (i.e., a sunk cost), does not change the utility of the decision to continue or end the romantic relationship. Similarly, the amount of money a person has invested in obtaining advice from a financial consultant (i.e., a sunk cost), does not alter the utility of the decision to either follow or ignore that advice. Thus, a normative decision would be based purely on the utility of continuing or ending the romantic relationship, or following or ignoring the advice (and not other information, such as the presence or degree of a sunk cost).

Additionally, normative decision making is also said to be characterized by two criteria: coherence or correspondence (Reyna \& Farley, 2006). Coherence refers to decision-making processes that are consistent with logic and follow rules applied across multiple scenarios (for instance, decision-making should follow the rule of transitivity - if a person prefers $\mathrm{X}$ over $\mathrm{Z}$, and $\mathrm{Z}$ over $\mathrm{Y}$, then they should also prefer $\mathrm{X}$ over $\mathrm{Y}$ ). On the other hand, the correspondence view of normative decision-making suggests that judgments and decisions that produce favorable outcomes in the "real world" are normative. Thus, while coherence defines normatively correct 
decision making in terms of processes, correspondence characterizes normatively correct decisions more in terms of the outcomes of those processes (Reyna \& Farley).

The use of analogous sunk scenario vignette pairs in the present study minimizes the effects of irrelevant information by holding information in the decision-making context that isn't related to the presence or absence (or amount) of the sunk cost constant. Moreover, the sunk cost scenario vignette pairs used in this study involve simple everyday scenarios, which should be easy to understand, and the types of sunk costs are simple and in amounts that most people are familiar with (i.e., investments of several dollars or several weeks or months of time). Thus, based on the normative models described here, there should be no reason for a participant to base their final decision (i.e., the decision of whether to invest more or less compared to an analogous vignette) on anything other than the expected utility of continued investment, which is constant across vignettes. Moreover, since the scenarios described in the vignettes are analogous across pairs, the rules that govern decision-making, as well as the potential outcomes of those decisions, should be identical across vignette pairs. Prior research has noted that human decision making is not always normative based on this model, a pattern that has been referred to as the “normative/descriptive" gap (Stanovich \& West, 1999; 2000). The tendency of individuals to demonstrate the sunk-cost fallacy as evidenced in previous studies (see Strough et al., 2011a for a review) is an example of this normative/descriptive gap.

Similar to the age differences in the sunk-cost fallacy, older adults also make normatively correct decisions more frequently than younger adults when responding to hypothetical sunk costs (Strough et al., 2008; 2011b). This study assesses whether older adults make normatively correct decisions more frequently than younger adults when considering two analogous 
hypothetical scenarios, one with a previously committed sunk cost, and the other without a sunk cost (Strough et al., 2008; 2011b).

Absolute vs. proportional investments. When investments are in the form of money, the sunk-cost fallacy may be more or less likely to occur based on whether the initial investment is an absolute amount, or is a proportion of a budget. Garland and Newport (1991) suggest that it is the relative proportion of the initial investment compared to an overall budget - rather than the absolute value of the initial investment- that has an impact on whether people demonstrate the sunk-cost fallacy. Specifically, when the initial investment is a large proportion of a budget, people are more likely to demonstrate the sunk-cost fallacy than if the investment is a small proportion of the budget. The absolute value of the initial investment, however, may have little or no impact on the likelihood that the fallacy will be demonstrated (Garland \& Newport). The present study directly compares a measure of the sunk-cost fallacy in which sunk costs are presented as a proportion of a budget to a measure in which the sunk costs are absolute values.

Types of investments. People demonstrate the sunk-cost fallacy when investing various types of resources; including money, time, and physical effort. Additionally, the way in which the fallacy is demonstrated might differ depending on which resources are being invested. For instance, people may demonstrate the sunk-cost fallacy following an initial investment of time (Navarro \& Fantino, 2009), although the fallacy may occur more reliably when money is invested than when time is invested (Soman, 2001). Moreover, the sunk-cost fallacy can occur when the initial investment consists of a resource that is different from later investments - an initial monetary investment may lead to later investments of time and other resources (e.g., Staw \& Hoang, 1995). For example, people may be more likely to follow advice that they have paid to receive than advice that was inexpensive or provided to them for free (Gino, 2008). Indeed, when 
people make initial investments in the form of money, they may be more likely to continue investing additional time (rather than additional money) in an effort to salvage the initial monetary investment. However, when people make initial investments of time, they appear to be more likely to continue investing additional money, rather than additional time (Heath, 1995). This study includes a comparison of two measures of the sunk-cost fallacy; the first of which involves initial and continued investments of different resources, the second of which involves a scenario in which the initial and continued investments are the same, and another scenario in which they differ. Specifically, the first measure in this study involves initial investments of money used to purchase advice, followed by continued investments in the form of the degree to which the person follows that advice. The second measure in this study involves a scenario in which the initial investment is hypothetical money and the continued investment is hypothetical time and different scenario in which both the initial and continued investments are in the form of hypothetical time.

\section{Implications of the Sunk-Cost Fallacy}

The implications of the sunk-cost fallacy may be relatively trivial (continuing to watch a boring movie just because one has paid to see it, for instance; Strough et al., 2011b), or of significant consequence (continuing to invest millions of dollars in the development of a product because of prior investments; Keil et al., 1995). However, the sunk-cost fallacy does not necessarily lead to negative outcomes. For example, if a person pays a gym membership fee, they may exercise more often than they would if the membership had been free. Indeed, it may be possible to develop interventions that capitalize on peoples' decision-making biases such that they are more likely to engage in health-promoting behaviors (Thaler \& Sunstein, 2008). The measures of the sunk-cost fallacy in this study involve relatively trivial consequences for 
demonstrating the fallacy that are similar to the types of consequences people might encounter in everyday life (e.g., losing a few dollars or continuing a hobby that one is bored with; Strough et al., 2011b).

\section{Potential Explanations of Investment Behaviors and the Sunk-Cost Fallacy}

Why do people demonstrate the sunk-cost fallacy, and why does the frequency with which it is demonstrated differ across people and types of resources? A variety of processes may be involved (see Strough et al., 2011a for a review); some people may demonstrate the fallacy in an effort to avoid wasting the initial investment (Arkes, 1996), particularly if the initial investment was large (Bornstein \& Chapman, 1995). Alternatively, people may demonstrate the sunk-cost fallacy because of social pressure to avoid acknowledging that the initial investment was a poor decision (Fox \& Staw, 1979). Indeed, people appear to be more likely to demonstrate the sunk-cost fallacy when they are personally responsible for the initial investment than when they are not (Staw, 1976). Each of these explanations is related to an assumption that people demonstrate the sunk-cost fallacy because of an attempt at self-justification- they try to prove to themselves or others that their decision to invest or continue investing was rational (Staw, 1981).

Another possible explanation for why people commit the sunk-cost fallacy is that people may view a particular product or course of action differently based on whether they have made an initial investment related to the product or course of action. For instance, people may have an impression that a course of action has an inflated probability of success as a consequence of making an initial investment (Arkes \& Blumer, 1985). For instance, a person may assume that a product a company is developing will have a higher probability of selling successfully if the company has already made a large investment in the product's development. However, if the 
company has only made a small investment in the product's development, the same person may assume that the same product has a lower probability of selling successfully (Arkes \& Hutzel, 2000). This study assesses peoples' perceptions of advice that they purchase with either smaller or larger sunk costs. Specifically, this study assesses whether people perceive advice that was purchased for a small amount of money to be of lower quality than advice that was purchased for a larger amount, and whether these perceptions relate to the sunk-cost fallacy.

Characteristics of the decision maker might also be related to the likelihood that the sunkcost fallacy will be demonstrated. For instance, Gino (2008) found that people who chose to pay a fee for advice were more likely to follow that advice than people who chose to receive the same advice, but who received it for free. One interpretation of Gino's finding is that the people who chose to pay for advice did so because they were less confident in their knowledge of the topic at hand, which led them to be more inclined to pay for and follow the advice than the people who chose to receive advice, but who got it for free. Gino tested this interpretation by comparing people who chose to purchase advice with those who chose not to purchase advice, and found that people who chose to purchase advice were just as confident in their understanding of the topic at hand than those who chose not to purchase advice. This study, similar to Gino's, also assesses whether people who are less confident in a topic are more likely to invest money to receive advice for that topic.

Individual differences in education and intelligence might also relate to the likelihood of demonstrating the sunk-cost fallacy. Some studies have shown that measures of academic aptitude, such as the SAT, are predictive of who is likely to demonstrate the sunk-cost fallacypeople with lower scores are more likely to demonstrate the fallacy than people with higher scores (Larrick et al., 1993; Stanovich \& West, 1999). Indeed, people who have the cognitive 
capacity to create a mental budget of available and committed resources may be less susceptible to demonstrating the sunk-cost fallacy (Heath, 1995). However, other research suggests that there is no relation between peoples' susceptibility to the sunk-cost fallacy and cognitive ability (Stanovich \& West, 2008) or their education (Arkes \& Blumer, 1985; Strough et al., 2008). Similarly, other studies have suggested that there is either no relation, or only a modest relation between peoples' resistance to sunk costs and their cognitive ability (Bruine de Bruin et al., 2007; Strough et al.). Thus, the relation between cognitive abilities and the likelihood of demonstrating the sunk-cost fallacy is still being explored. The present study included two measures of the sunk-cost fallacy, each of which required participants to consider numerical content including real and hypothetical monetary values, hypothetical time values, and estimates of caloric content of food items. This study assessed whether peoples' performance on two measures of the sunkcost fallacy related to their self-reported numerical ability.

A decision maker's tendency to employ certain decision-making rules may also relate to the sunk-cost fallacy. The employment of some heuristics that can be useful in everyday decision-making may lead to the sunk-cost fallacy when they are over-generalized to scenarios in which investment does not lead to a payoff (Arkes \& Ayton, 1999). For instance, many people employ the heuristic that prior investment generally correlates with later benefits, (Gigerenzer, Czerlinski, \& Martignon, 1999). Similarly, people are more likely to assume that scenarios with high sunk costs will produce greater returns than scenarios with low sunk costs (Heath, 1995). Lastly, most people are averse to losses, and this aversion can relate to a number of decisionmaking biases (see Tverskey \& Kahneman, 1991 for a review). Specifically, if a person views sunk costs as a loss or waste, they may focus more on the sunk costs when making decisions, which may lead to the sunk-cost fallacy (Arkes \& Ayton; Arkes \& Blumer, 1985; Soman, 2004). 
An approach that may provide a comprehensive explanation of why the sunk-cost fallacy is demonstrated, and how it relates to loss aversion and elements of decision-making heuristics, is prospect theory (Kahneman \& Tversky, 1979). Garland and Newport (1991) note three elements of prospect theory that help to explain why the sunk-cost fallacy is demonstrated. First, when considering a decision, people consider outcomes in terms of gains and losses in comparison to a point of reference. When an initial investment has been made and there have been no returns (as in the case of the sunk-cost fallacy), it is likely to be viewed as a loss, which most people will attempt to avoid (Garland \& Newport; Kahneman \& Tversky), and which may be related to an increased likelihood of demonstrating the sunk-cost fallacy (Soman, 2004). Indeed, Heath (1995) notes that people tend to set mental budgets aimed at investing less than the total available return on the investment — similar to basing a decision on gains and losses with respect to a reference point. In this study, people chose whether to invest money to receive advice that could be used to make a profit; the potential profit was larger than the amount invested, although no profit was guaranteed (and it was possible that some, or all of the investment, could be lost). Additionally, in the present study, participants' aversion to losing money was assessed and the relations between monetary loss aversion, decision to invest money, and the sunk-cost fallacy were assessed.

Secondly, when deciding between two or more courses of action, people weight the options with certain outcomes more heavily than those with probable outcomes. Terminating continued investment following an initial investment creates a scenario in which a perceived loss is certain, whereas a perceived loss is less certain if investment continues (Garland \& Newport, 1991; Kahneman \& Tversky, 1979). People are more likely to continue investing following initial sunk costs when they do not have information regarding the expected return on investment 
(when a future loss is not certain), but are less likely to continue investing if they know that continued investment will not produce profit (Heath, 1995). In this study, participants were aware that any money they chose to invest would be deducted from their total available funds - a certain outcome. Additionally, participants were aware that it was possible to earn back the money they had spent, and possibly make a profit—a possible outcome. Moreover, participants in this study did not receive information about the amount of profit they had earned (if any) until after they finished making decisions related to how much continued investment they were willing to make (i.e., to what extent they would follow the advice they had paid for). Thus, in the present study, participants experienced the classic sunk cost scenario: an initial investment, once made, was a certain outcome, while the outcomes of continued investment were uncertain.

Lastly, there is a convex value function for losses, which means that as more resources are invested, losses become less salient (Garland \& Newport, 1991; Kahneman \& Tversky, 1979). This would suggest that the more one has invested in the past, the more one will invest in the future; an issue of degree of overinvestment - also referred to as escalation of commitment (Heath, 1995). This study assesses the relation between monetary loss aversion and likelihood of investing money, and also assesses degree of overinvestment.

\section{Explanations of Age Differences in the Sunk-Cost Fallacy}

There may be multiple mechanisms underlying the age differences in how frequently younger and older adults demonstrate the sunk-cost fallacy. One possibility (suggested by Strough et al., 2008) is that age differences in how people attend to positive and negative information — such as information regarding gains and losses - influences the decisions they make regarding sunk costs. Because sunk costs are irretrievable, people may view them 
negatively as lost resources. Although research using primarily younger adult participants has shown that people tend to focus more on negative than positive information (Baumeister, Bratslavsky, Finkenauer, \& Vohs, 2001), and losses exert more influence than gains over the choices that people make (Kahneman, Knetsch, \& Thaler, 1990; Tversky \& Kahneman, 1991), older adults tend to be more accurate than younger adults in how they reason about gains and losses. In particular, older adults tend to weigh gains and losses more equally than younger adults (Wood, Busemeyer, Koling, Cox, \& Davis, 2005). Additionally, although younger and older adults are equally likely to take risks to avoid a loss (Weller, Levin, \& Denburg, 2009), older adults are less influenced by information that is framed in terms of losses than younger adults (Mikels \& Reed, 2009). Thus, younger adults may respond differently to monetary investments such as sunk costs than older adults if the investments are viewed as losses.

Indeed, younger adults place greater weight on negative information (they are subject to a negativity bias; i.e., Baumeister et al., 2001), and place greater weight on losses compared to wins more than older adults (Wood et al., 2005). Conversely, older adults exhibit a positivity effect by focusing more on positive information than negative information; a pattern that tends to increase with age (Carstensen \& Mikels, 2005; Mather \& Carstensen, 2005). For instance, older adults are less likely to remember negative images, and recall a higher ratio of positive to negative images than middle-aged, or younger adults (Charles, Mather, \& Carstensen, 2003). Older adults are also less averse to loss-framed risky decisions (Mikels \& Reed, 2009), and exhibit less neural activity in response to monetary losses than younger adults (Samanez-Larkin et al., 2007). Similarly, compared to younger adults, older adults exhibit less response in the amygdala (an area of the brain associated with emotion) when exposed to negative images, and a greater response when exposed to positive images - this age difference may be related to the 
aforementioned decrements in memory of negative images that older adults experience (Charles et al., 2003; Mather et al., 2004).

Possibly because they are more focused on, or have a stronger aversion to losses, younger adults may be more likely than older adults to follow courses of action that they assume will allow them to circumvent or recover losses. Thus, if younger adults view sunk costs as losses, they may be more likely than older adults to continue investing in hopes that they will recover or capitalize on the sunk costs—-such an approach would increase younger adults' likelihood of demonstrating the sunk-cost fallacy. Similarly, in scenarios where younger adults are faced with a decision to either invest or retain money, they may be less likely to commit an investment than older adults if they view the investment as a potential loss.

Why are older adults more focused on positive information, and less concerned with negative information than younger adults? Older adults may experience the positivity effect in part because their future time perspective is more limited than younger adults'. Future time perspective is a person's awareness and perception of the temporal proximity of impending deadlines. For younger adults, the future is relatively expansive and future time perspective is expansive. However, older adults' impending mortality creates a deadline which limits their future time perspective (Lang \& Carstensen, 2002). When people experience a more limited future time perspective, they tend to focus more on emotional experiences, particularly positive emotional experiences — which may play a role in the aforementioned positivity effect that older adults experience (Carstensen, Isaacowitz, \& Charles, 1999; Mather \& Carstensen, 2005). This study assesses whether younger and older adults' future time perspective relates to monetary loss aversion. Moreover, this study assesses whether monetary loss aversion accounts for age differences in demonstration of the sunk-cost fallacy. 


\section{Loss Aversion, Delay Discounting of Gains and Losses, and Monetary Tradeoffs}

As previously mentioned, age differences in attention to positive and negative information (Carstensen \& Mikels, 2005; Charles et al., 2003; Mather \& Carstensen, 2005; Mather et al., 2004) and loss aversion (Wood et al., 2005) may help explain the age differences in the sunk-cost fallacy (e.g. Strough et al., 2008). Loss aversion is a phenomenon that has been studied in humans using a number of methods, including correlational and experimental assessments of how people buy and sell goods in both real and hypothetical scenarios (e.g. Genesove \& Mayer, 2001; Kahneman et al., 1990; Thaler, Tversky, Kahneman, \& Schwartz, 1997). For instance, Genesove and Mayer analyzed existing real estate market data and concluded that real estate sellers who were subject to monetary losses exhibited loss aversion by setting higher asking prices than sellers who were not subject to monetary losses. Others have studied loss aversion using tasks involving the trading of goods that show that people are reluctant to give up items that they own — a phenomenon referred to as “....an endowment effect...” (Kahneman et al., 1990, p. 1342). For instance, Kahneman and colleagues have demonstrated that people perceive an item that they possess to be more valuable than an item that they do not possess, and are therefore less likely to trade the first item for the second, even when the values of the items are equal. Although some researchers have studied peoples' willingness to buy, sell, and trade real goods, others have assessed loss aversion by asking participants to consider hypothetical investment scenarios involving the buying of hypothetical stocks (Thaler et al., 1997).

One approach to studying how people avoid losses is to compare how often they prefer small immediate monetary gains over larger delayed gains to how often they prefer larger delayed monetary losses over smaller immediate losses. These two phenomena are referred to as 
delay discounting of gains and losses, respectively. The section that follows discusses how people prefer smaller immediate rewards and larger delayed losses, but it should be made clear that this is not the focus of this study — measures of peoples' preferences for immediate versus delayed gains and losses were employed to assess loss aversion in the this study, and not necessarily delay discounting.

Much of the literature regarding peoples' preferences for immediate versus delayed gains and losses focuses on whether people prefer to receive a small gain immediately or a larger gain after a time delay (Baker, Johnson, \& Bickel, 2003; Green, Fry, \& Myerson, 1994). However, some studies have also addressed whether people prefer a larger delayed loss or a smaller immediate loss (Baker et al.; Benzion, Rapoport, \& Yagil, 1989). Indeed, it is important to note that comparing peoples' preference for immediate versus delayed gains to their preference for immediate versus delayed losses usually shows that the direction of the preference changes. Specifically, people generally prefer small immediate gains and large delayed losses over large delayed gains and small immediate losses (Baker et al.; Benzion et al.).

Tasks that measure delay discounting of gains and losses do not necessarily provide information regarding loss aversion. However, one can assess loss aversion by comparing the degree to which a person prefers a small immediate gain to the degree to which they prefer a large delayed loss. Specifically, an experimenter can have participants indicate their preferences for immediate versus delayed gains and losses across a variety of time delays. For instance, a participant might indicate whether they prefer a small immediate reward or a larger reward after a time delay of a week, a month, and a year. The experimenter can then plot two curves; one of the participants' preferences for immediate versus delayed gains as a function of delay, and the other for immediate versus delayed losses as a function of delay. The experimenter then 
calculates the area under each curve (Myerson, Green, \& Warusawitharana, 2001). Loss aversion is assessed by subtracting the area under the losses curve from the area under the gains curvelarger values indicate loss aversion. Using this approach, one can assess individual differences in loss aversion, and thereby address a mechanism that may underlie age differences in demonstration of the sunk-cost fallacy.

More specifically, a large gain is generally more desirable than a small gain, and a small loss is more desirable than a large loss. However, as the delay prior to a gain or loss increases, so does the potential for never experiencing that gain or loss. A highly loss averse person would choose smaller immediate gains, and larger delayed losses, because an immediate (albeit smaller and thus less-desirable) gain is more certain than a delayed (but larger) gain, and a delayed (albeit larger and thus less-desirable) loss is less likely to occur than an immediate (but smaller) loss. Put differently, a highly loss averse person would want to minimize the chances of never receiving (i.e., losing) a gain, while maximizing the chances of never experiencing a lossregardless of the magnitude of the gain or the loss.

Prior research has suggested that older people choose large delayed gains over small immediate gains more frequently than younger people (i.e., younger adults discount delayed gains more than older adults; Green et al., 1994; Harrison, Lau, \& Williams, 2002; Löckenhoff, O’Donoghue \& Dunning, 2011; Reimers, Maylor, Stewart \& Chater, 2009; Steinberg, Graham, O’Brien, Woolard, Cauffman \& Banich, 2009; Whelan \& McHugh, 2009). However, findings related to age differences in delay discounting are limited and sometimes mixed or ambiguous (e.g., Harrison et al.), with some studies failing to find age effects (e.g., Chao, Szrek, Pereia, \& Pauly, 2009), and others finding an age effect with older adults discounting delayed gains more than younger adults (e.g., Read \& Read, 2004). Additionally, many studies that have examined 
age differences in delay discounting involved different parameters (i.e., different monetary values, different currencies, different delay values; e.g. Green et al. vs. Löckenhoff et al. vs. Whelan \& McHugh), making direct comparisons of different studies difficult. Moreover, to date, few studies have investigated age differences in the delay discounting of losses, but there is some evidence that delay discounting of losses does not differ significantly with age (Löckenhoff et al.).

However, research showing that older adults weigh gains and losses more equally than younger adults (Wood et al., 2005) suggests that younger and older adults may differ in how they choose between immediate and delayed losses. Specifically, older adults' decisions about delayed versus immediate losses may be more similar to their decisions about delayed versus immediate gains than younger adults. Moreover, because older adults exhibit a positivity effect by focusing more on positive information than younger adults (Carstensen \& Mikels, 2005; Mather \& Carstensen, 2005), they may be more likely to focus on larger delayed gains (i.e., positive information), while paying less attention to the delay before the gain (i.e., negative information), whereas younger adults may focus more on the delay than the amount of the delayed gain. This may lead older adults to choose larger delayed gains more frequently than younger adults.

If older adults choose large delayed gains over smaller immediate gains, and/or choose smaller immediate losses over large delayed losses more frequently than young adults, it would suggest that they are less loss-averse than younger adults. Such a finding might provide an explanation for why older adults demonstrate the sunk-cost fallacy less frequently than younger adults - particularly if loss aversion mediates the relation between age and the sunk-cost fallacy. 
Another approach to assessing peoples' aversion to losing money is to have them make a series of dichotomous choices between hypothetical tradeoff scenarios, each of which involves a possibility of either gaining, or losing a certain amount of money (e.g., Fennema \& Van Assen, 1999). More specifically, if a person is asked to choose between two hypothetical scenarios, the first of which involves an equal chance (i.e., a 50\% chance) of either gaining $\$ 1000$ or losing $\$ 5$, the second of which involves an equal chance of either gaining $\$ 1500$ or losing $\$ 5$, the rational choice is to choose the second scenario, since the potential gain is larger, and the potential loss is equal to the first scenario. However, if the potential loss value in the second scenario is increased on subsequent trials, then the larger potential gain of the second scenario becomes progressively less desirable in comparison to the first scenario (which has a smaller potential gain, but also a smaller potential loss). This approach was adapted from Fennema and Van Assen, and used in the present study-loss aversion was assessed based on the loss value that was present in the second scenario when a participant switched preference from the second scenario and chose the first scenario with the smaller potential gain and loss (i.e, the participant's indifference point). Indifference points that occurred at lower potential loss values in the second scenario indicated greater loss aversion than indifference points that occurred at larger potential loss values (i.e., people who tolerated larger potential losses before reaching an indifference point were less loss averse than people who tolerated smaller potential losses; see Appendix D). Thus, in the present study, age differences in loss aversion were assessed using a delay discounting of gains and losses task adapted from Green and colleagues (1994), and by using a tradeoff task adapted from Fennema and Van Assen (1999).

\section{Limitations of Prior Research}


Multi-method approach. This study involved two measures of the sunk-cost fallacy; one measure (adapted from Gino, 2008) allowed participants to decide whether or not to commit a real monetary investment, the other measure (Strough et al., 2008; adapted from Frisch, 1993) involved scenarios where participants were told that they either had or had not made an initial hypothetical investment. The task used by Gino was similar to the one used in this study, in that participants in this study had the opportunity to purchase advice for either a less expensive, or a more expensive fee (i.e., a smaller or a larger sunk cost). Similar to Gino's method, the sunk-cost fallacy in this study was measured by the degree to which participants followed the less expensive advice, compared to the degree to which they followed the more expensive advice. Specifically, in this study, a person demonstrated the sunk-cost fallacy when they followed the more expensive advice more closely than the less expensive advice (the less expensive and more expensive advice were the same, and thus the cost of the advice should not have related to how closely people followed the advice). However, there are several differences between the method used in this study and the method used by Gino - namely, this study assessed age differences in monetary investment, demonstration of the sunk-cost fallacy, and loss aversion as a potential explanatory variable underlying the sunk-cost fallacy, whereas Gino did not.

Real vs. hypothetical sunk costs. The majority of prior lab-based research investigating monetary investments and the sunk-cost fallacy in humans have employed procedures involving hypothetical investments and sunk cost scenarios (e.g. Arkes, 1996; Arkes \& Hutzel, 2000; Beeler \& Hunton, 1997; Bornstein \& Chapman, 1995; Bruine de Bruin et al., 2007; Fox \& Staw, 1979; Garland \& Newport, 1991; Keil et al., 1995; Larrick et al., 1993; Stanovich \& West, 1999; Staw, 1976; Soman, 2001; Strough et al., 2008; Tan \& Yates, 1995). The most recent studies of 
the effects of sunk costs (e.g., Coleman, 2010; Molden \& Hui, 2011) have also used self-report measures describing hypothetical sunk cost scenarios.

A relatively small number of studies have involved real, rather than hypothetical, sunk costs. Real monetary sunk costs were assessed by Arkes and Blumer (1985), although their study was conducted in the field, rather than in a laboratory environment. Navarro and Fantino (2009) conducted a study that employed a behavioral task involving sunk costs, but the sunk costs and investments were in the form of time, not money. Similarly, other studies (e.g., Cunha \& Caldieraro, 2009; Soman, 2001) have investigated the effects of sunk costs that were in the form of time investments. Navarro and Fantino (2005) studied the sunk-cost fallacy using a behavioral task involving real monetary rewards. However, the investments that participants committed in Navarro and Fantino's study were in the form of time and effort, and not money, and the participants engaged in a basic operant task. Staw and Hoang (1995) used real performance data to assess demonstration of the sunk-cost fallacy, but their study used data collected in the field and a correlational design instead of an experimental design. Lab-based research procedures generally offer the opportunity for greater control over the phenomena under study in comparison to research that is conducted based on data gathered in the field. Thus, although labbased research procedures may or may not produce data that is representative of phenomena that occurs outside of laboratory environments, the results that are observed in laboratory settings are often more reliable than those observed outside of laboratory settings (Goodwin, 2008). Therefore, the investigation of how people make decisions in sunk cost scenarios in laboratory settings is warranted.

Thus, current knowledge is limited regarding how people make real monetary investments in laboratory settings and whether people demonstrate the sunk-cost fallacy when 
the sunk costs are real, rather than hypothetical monetary investments. The potential for differences between peoples' self-reports and actual behavior, particularly when related to monetary investments (e.g. Blumenschein, Johannesson, Blomquist, Lijas, \& O’Conor, 1998; Blumenschein, Johannesson, Yokoyama, \& Freeman, 2001; Botelho \& Pinto, 2001; Cummings, Harrison \& Ruström., 1995; Johannesson, Liljas, \& Johansson, 1998; Loomis, Brown, Lucero, \& Peterson, 1997; Neill, Cummings, Ganderton, Harrison, \& McGuckin, 1994; Slovic, 1969) underscores the importance of studying investing behavior and demonstration of the sunk-cost fallacy using real monetary values. Specifically, peoples' hypothetical willingness to pay for goods may not reflect their actual purchase decisions (Bothello \& Pinto; Johannesson et al.; Neil et al.), and may overestimate people's purchase decisions (Cummings et al.; Loomis et al.). This study assessed age differences in how people commited investments and demonstrated the sunkcost fallacy in a laboratory environment when the investments and potential returns were in the form of real money. Moreover, the present study compared a measure of the sunk-cost fallacy that involved real sunk costs and potential returns to a measure that involved hypothetical sunk costs and potential returns.

Participant-chosen investments. Few studies related to the sunk-cost fallacy have allowed participants to actively choose whether or not to commit an initial investment. Instead, most prior research involved procedures that informed participants that an initial investment had already been committed (either by the participant, or by a character in a vignette that the participant was reading). That is, participants were not given the option of choosing whether or not to commit the initial investment (e.g., Arkes, 1996; Arkes \& Hutzel, 2000; Bornstein \& Chapman, 1995; Bruine de Bruin et al., 2007; Coleman, 2010; Garland \& Newport, 1991; Heath, 1995; Keil et al., 1995; Larrick et al., 1993; Soman, 2001; Stanovich \& West, 1999). The benefit 
of not allowing participants to choose whether to commit an initial investment in research related to the sunk-cost fallacy is that it controls for a confounding of likelihood of investing and demonstration of the sunk-cost fallacy. However, the drawback is that in natural settings, people typically choose whether or not to commit an initial investment before they face sunk-cost scenarios; it is uncommon for people to be told by an outside entity that their resources have been invested for them without their consent. Thus, the ecological validity of methods that do not allow participants to choose whether to commit an initial investment may be limited; particularly given that peoples' reports of their willingness to commit hypothetical monetary investments may overestimate their willingness to commit real monetary investments (Blumenschein et al., 1998).

Some prior studies (e.g. Beeler \& Hunton, 1997; Fox \& Staw, 1979; Staw, 1976) allowed participants to choose how to commit investments, but the investments were entirely hypothetical, and participants chose between several options of where to allocate the investment, and not necessarily whether or not to make an investment. Thus, current knowledge is limited regarding how people make real monetary investments and demonstrate the sunk-cost fallacy in laboratory settings when they are actively involved in choosing whether or not to invest, rather than being passively told whether or not they have invested. The present study compared a measure of the sunk-cost fallacy that allowed people to choose whether or not to commit initial investments to a measure that did not. Moreover, although there have been numerous studies of peoples' willingness to pay for a variety of goods and services (e.g., Blomquist \& Whitehead, 1998; Bushong, King, Camerer, \& Rangel, 2010; Tambour \& Zethraeus, 1998), this study was novel in that it examined age differences in peoples' willingness to pay, which is a prerequisite to demonstration of the sunk-cost fallacy. 
Monetary amounts. Many prior studies of investment behavior and the sunk-cost fallacy have involved relatively large hypothetical monetary values. For instance, some research has asked participants to consider investments of hundreds (Bornstein \& Chapman, 1995), tens of thousands (Beeler \& Hunton, 1997), hundreds of thousands (Arkes \& Hutzel, 2000), and even millions of dollars (Fox \& Staw, 1979; Garland \& Newport, 1991; Keil et al., 1995; Molden \& Hui, 2011; Staw, 1976). Many of these studies have asked participants to consider hypothetical investments from another person's perspective (e.g., Bornstein \& Chapman), or play the role of a corporate official considering hypothetical business investments (e.g., Arkes \& Hutzel; Beeler \& Hunton, Fox \& Staw; Garland \& Newport, Keil et al.; Molden \& Hui; Staw; van Putten, Zeelenberg, \& van Dijk, 2010). Thus, current knowledge regarding whether people demonstrate the sunk-cost fallacy when sunk costs are real monetary values in amounts that are typically encountered in everyday life is limited. This study is designed to address this issue by including real monetary sunk costs in amounts that most people typically encounter in everyday life. Moreover, the investments and potential returns in this study are made and received directly by the participants, rather than being presented as part of a hypothetical business scenario.

\section{Statement of the Problem}

The sunk-cost fallacy is a bias in which people invest more because of an initial investment, and is an example of irrational decision-making. Older adults are less likely to demonstrate the sunk-cost fallacy than younger adults (see Strough et al.2011a for a review). Studying the mechanisms that may explain age differences in susceptibility to the sunk-cost fallacy will provide a more comprehensive understanding of the causes of the sunk-cost fallacy, as well as the parameters under which it occurs. The effects of committing the sunk-cost fallacy may be positive or negative, and may be relatively trivial or of serious consequence, depending 
on the nature of the investments. Thus, it is relevant to understand how and why the sunk-cost fallacy occurs so that it can be prevented when its outcomes are negative, or be facilitated when its outcomes are positive. This study assessed age differences in how frequently people demonstrated the sunk-cost fallacy, age differences in the degree to which people who demonstrated the fallacy overinvested, as well as age differences in monetary loss aversion and whether loss aversion served as a mechanism underlying age differences in demonstration of the sunk-cost fallacy. Furthermore, this study assessed whether an established self-report measure of the sunk-cost fallacy (i.e., Frisch, 1993; Strough et al., 2008; 2011b) related to peoples' behavior when real rather than hypothetical sunk costs were at stake. Additionally, although prior research has studied people's willingness to pay for a variety of goods (e.g., Blomquist \& Whitehead, 1998; Bushong et al., 2010), little is known regarding whether younger and older adults invest money differently when the investments and potential future returns are real, rather than hypothetical. This study addressed whether there were age differences in how younger and older adults invested money in information regarding a topic that could be used to earn more money. Also, this study investigated how peoples' knowledge of a topic and confidence in their knowledge related to the likelihood of investing in information regarding that particular topic.

\section{Research Questions and Hypotheses}

\section{Research Question 1}

Are there age differences between younger and older adults in how often they demonstrate the sunk-cost fallacy by weighting expensive advice more heavily than inexpensive advice?

\section{Hypothesis 1}


Younger adults will demonstrate the sunk-cost fallacy by weighting expensive advice more heavily than inexpensive advice more often than older adults.

\section{Research Question 2}

Are there age differences in degree of overinvestment?

\section{Hypothesis 2}

Older adults who demonstrate the sunk-cost fallacy will overinvest less than younger adults who demonstrate the sunk-cost fallacy by weighting expensive advice less heavily than younger adults.

\section{Research Question 3}

Are there age differences in loss aversion?

\section{Hypothesis 3}

Older adults will display less loss aversion than younger adults on a delay discounting of gains and losses task and on a tradeoff task.

\section{Research Question 4}

Are people who choose to purchase advice regarding an estimate they are making any more or less confident in their estimates before receiving advice than people who choose not to purchase advice?

\section{Hypothesis 4}


People who choose to purchase advice will not be any more or less confident in their estimates prior to receiving advice than people who choose not to purchase advice.

\section{Research Question 5}

Do the age differences in loss aversion described in Hypothesis 4 mediate the age differences in demonstration of the sunk-cost fallacy described in Hypothesis 1 ?

\section{Hypothesis 5}

The age differences in loss aversion on both the delay discounting and the tradeoff tasks described in Hypothesis 4 will mediate the age differences in demonstration of the sunk-cost fallacy described in Hypothesis 1.

\section{Method}

\section{Design}

This study used a cross-sectional sample of younger and older adults to assess age differences in six primary dependent variables: demonstration of the sunk-cost fallacy, loss aversion, estimate confidence, advice purchase, degree of overinvestment, and normatively correct decisions. This study employed a 2 (sunk cost: large, small) X 2 (age group: younger adult, older adult) quasi-experimental design. Sunk cost was a within-subject variable, and age group was a between-subject variable.

\section{Participants}

The sample was comprised of 50 college-aged younger adults (18-31 years old, $M=$ $20.44, S D=2.52,68 \%$ women, $96 \%$ never married, $M$ years of education $=13.60)$ and 50 
community-dwelling older adults ( $60-85$ years old, $M=69.74, S D=6.85,56 \%$ women, $2 \%$ never married, $M$ years of education $=15.91$; see Table 2). Younger adult participants were recruited from the undergraduate student body at West Virginia University through advertisements around campus, in-class announcements, and via the Sona Ltd. research participation system. Older adult participants were recruited from the community of Morgantown, West Virginia, and Garrett and Allegany Counties in Marylandthrough email advertisements and over the phone. Older adults were recruited by a snowballing procedure in which email advertisements were sent to listservs and individuals. Email advertisements asked recipients to forward the advertisement to other older adults who might be interested in participating. The initial email addresses and listservs that were used were obtained from personnel at the Flowery Vale senior center in Accident, Maryland. All participants were provided with $\$ 5$ compensation and had an opportunity to earn up to an additional $\$ 10$ during the study.

\section{Procedure}

All participants provided informed consent prior to participation. Participants chose to attend a testing session at one of two locations - a laboratory at the Life Sciences Building at West Virginia University, or a testing session at a time and location that was convenient for them. All younger adults participated at the laboratory. All older adults chose to participate either at their homes $(n=39)$, or at a community center $(n=11)$. All participants completed the study in a quiet environment with minimal distraction. Participants were able to choose the dates and times for their testing session. Additionally, participants were told that they would receive a $\$ 5$ show-up fee, and would have an opportunity to earn more money in exchange for their 
participation. Participants were informed that the purpose of the study was to obtain information regarding how people think about food.

Upon arrival at the testing session, participants were greeted by a trained research assistant who asked them to read and sign a consent form (see Appendix K). Participants were then seated in front of a computer and were told that the instructions for the study would appear on the computer (see Appendix A), and that they were to read the instructions carefully and ask the research assistant if they didn't fully understand any part of the instructions or the study. After reading the instructions, participants clicked a button to continue and the testing session began. The research assistant remained in the room for the duration of the testing session to clarify any issues for participants who did not understand any aspect of the instructions or testing session. Participants completed the entire procedure on the computer program, which was coded using Microsoft@ Visual Basic $® 2010$.

Participants first completed a Calorie Estimation Task that occurred in 4 phases adapted from Gino (2008; Study 1). Specifically, in each of the 4 phases of the task, participants read one of two lists of food items (see Appendix B) and provided calorie estimates for each food item on each list, as well as $90 \%$ confidence intervals for each of their estimates.

Participants began Phase 1 by completing the Calorie Estimation Task using Food Item List 1 (see Appendix B). Prior to beginning Phase 1, participants read instructions that told them that for each accurate calorie estimate for a given food item on Food Item List 1 - that is, an estimate that was within $10 \%$ of the actual caloric value of that food item - they would win a payment of $\$ 0.50$ cents. The instructions informed participants that any money that they won through accurate calorie estimates, up to $\$ 10.00$ would be added to their $\$ 5.00$ show-up fee. The 
instructions informed participants that "Any money that you win will be added to your $\$ 5.00$ show-up fee, and the total amount will be given to you after the study is complete." Participants did not receive any information regarding the accuracy of their estimates until debriefings were sent to them after all data collection was complete. Data collection for the present study took place between October 2010 and March 2011; final debriefings and payment were distributed in April, 2011. Thus, participants waited between 1 and 6 months after participating before receiving final debriefing and payment. Participants did not receive any information regarding the accuracy of their estimates or the caloric content of the food items in the Calorie Estimation Task until they received their final debriefing and payment.

Upon completion of Phase 1, Phase 2 began, in which participants once again received Food Item List 1 and read instructions asking them to complete the Calorie Estimation Task a second time. Phase 2 was identical to Phase 1 with one exception: in Phase 2, participants had the option of purchasing advice and read instructions that informed them they had the option of purchasing expert advice regarding the caloric values of all of the food items on the list for either $\$ 1.00$ (small price of advice condition), or for $\$ 3.00$ (large price of advice condition). Specifically, participants read the instructions: “...you now have the option of purchasing advice from an expert regarding the calorie values of every item on the food item list." prior to choosing whether to purchase advice.

Participants also read instructions which told them that any money that they spent and/or won was "real" in the sense that any money they spent or won was deducted or added to their $\$ 5.00$ show-up fee and the total would be paid to them after the study. Although not explicitly stated, the information provided in the instructions showed that participants who made accurate calorie estimates on all food items could earn up to $\$ 15$ if they didn't purchase advice, $\$ 14.00$ if 
they purchased advice in the small price of advice condition, $\$ 12.00$ if the purchased advice in the large price of advice condition, and $\$ 11.00$ if they purchased advice in both conditions. The instructions also informed participants that although purchasing advice would cost them money, the advice could be used to make more accurate estimates, thereby earning them money. In reality, no deductions were taken from the final amount that participants were paid at the end of the study; all participants were paid $\$ 5.00$, plus any money they won through accurate estimates, up to $\$ 10.00$. Deductions for advice purchasing were not taken from the final amount that participants were paid so that all participants could receive the same monetary benefit from participating, plus any earnings they received through accurate estimates. Therefore, participants had the opportunity to earn up to $\$ 15.00$ for participation, regardless of whether they purchased advice (although they were not made aware of this until they received a debriefing packet after the study was complete).

Participants chose whether or not to purchase advice; participants who purchased advice were able to view the aforementioned advice on the computer screen while they once again provided their own calorie estimates and $90 \%$ confidence intervals for each food item as they did in Phase 1. When a participant chose to purchase advice, a bold-font message which read "You have purchased advice for X dollars" appeared and remained on the screen while the participant made calorie estimates - the dollar amount reflected the price of advice condition (i.e. $\$ 1.00$ or \$3.00). Participants who did purchase advice responded to a measure of perceived quality of advice adapted from Gino (2008; see appendix A) after they finished making calorie estimates within that particular phase.

Participants then completed Phases 3 and 4, which were identical to Phases 1 and 2, respectively, but with two exceptions. First, Food Item List 2 was employed in Phases 3 and 4 
(as opposed to Food Item List 1). Secondly, in Phase 4, the amount required to purchase advice was different than in Phase 2. Specifically, order of price of advice condition (large/small) was counterbalanced so that sequence effects could be analyzed (Gino, 2008). Thus, there were 2 sequences (see Table 1); in sequence 1, Phase 2 served as the small price of advice condition (\$1.00 to purchase advice), while Phase 4 served as the large price of advice condition $(\$ 3.00$ to purchase advice). In sequence 2, Phase 2 served as the large price of advice condition ( $\$ 3.00$ to purchase advice) and Phase 4 served as the small price of advice condition ( $\$ 1.00$ to purchase advice).

After participants completed each of the 4 phases of the Calorie Estimation Task, they completed the Delay Discounting Loss Aversion Task adapted from Green and colleagues (1994; see Appendix C). Order of presentation of the conditions of the task (loss/gain condition) was counterbalanced.

Participants then completed a Tradeoff Loss Aversion Task adapted from Fennema and Van Assen (1999; see Appendix D). Following the Tradeoff Loss Aversion Task, participants completed the Subjective Numeracy Scale (SNS; Fagerlin, Zikmund-Fisher, Ubel, Jankovic, Derry, \& Smith, 2007; see Appendix E), the weight control sub-scale of the Food Choice Questionaire (FCQ; Steptoe, Pollard, \& Wardle, 1995; see Appendix F), an exploratory Health Habits Questionnaire (HHQ; see Appendix F ) a self-report measure of the sunk cost fallacy (Strough et al., 2008; see Appendix G), the Spendthrift-Tightwad Scale (STS; Rick, Cryder, \& Loewenstein, 2008; see Appendix H) and the Future Time Perspective Scale (FTPS; Carstensen \& Lang, 1996; see Appendix I). Lastly, participants completed a demographics form (see Appendix J). Participants were then asked to provide their mailing address and were told that 
compensation and a debriefing would be mailed to them. Participants were thanked for their time, given an opportunity to ask any remaining questions, and dismissed.

\section{Measures}

The measures are described below in the order in which they were presented to participants.

Calorie Estimation Task. This study employed an estimation task adapted from Gino (2008; study 1), which involved estimating certain values based on limited information. The primary difference between the task used in this study and the one employed by Gino were that Gino's participants estimated the dates of 30 historical events (15 events on 2 lists), whereas the task in this study involved estimating the caloric content of 30 food items (15 food items on 2 lists). Participants estimated the number of calories in different food items in this study (rather than dates of historical events as in Gino's study) because pilot data indicated that younger and older adults were equally accurate in their ability to estimate the caloric content of food items. Subsequent analyses showed that the younger and older adults in the present study were not significantly different in their accuracy of calorie estimates (see Exploratory question 1).

Additionally, Gino (2008; study 1) defined an "accurate" estimate as one that fell within 10 years of the actual date of the historic event, whereas an "accurate" estimate in the present study was defined as a calorie estimate that fell within $10 \%$ of the actual caloric value of the respective food item. Moreover, Gino's study differed from this one in that Gino's participants received $\$ 10.00$ for showing up and had an opportunity to earn $\$ .40$ cents for each accurate estimate, and could receive advice for free (i.e., a no sunk-cost condition), or for $\$ 4.00$ (i.e., a large sunk-cost condition). Participants in the present study, by contrast, received $\$ 5.00$ for 
showing up, and had an opportunity to earn $\$ .50$ cents for each accurate estimate, and could purchase advice for either $\$ 1.00$ (small price of advice condition), or $\$ 3.00$ (large price of advice condition). Also, the advice that participants could purchase in Gino's study originated from a person who was not an expert on the topic (i.e., they were not an expert in history), whereas the advice that participants in the present study could purchase originated from an expert (i.e., a dietician). The changes from Gino's study that were made for the present study were intended to improve the ecological validity of the present study. Specifically, when people pay money to receive advice, they generally pay for advice from a trained expert (e.g., advice from a doctor or attorney), whereas it is less common to pay for advice from a person who is not a trained expert (e.g., receiving advice from a friend). Moreover, advice that is obtained from an expert (e.g., from a financial consultant), is generally obtained through some type of formal business arrangement, and is typically not received for free.

Several changes were made to Gino's procedure before it was applied to the present study. Specifically, participants estimated calories instead of historic dates, participants received $\$ 5$ and earned $\$ .50$ cents for accurate estimates (instead of $\$ 10.00$ and $\$ .40$ cents, respectively), advice cost participants either $\$ 1.00$ or $\$ 3.00$ (instead of free advice or advice that cost $\$ 4.00$, respectively), and advice originated from an expert instead of from a non-expert. Potential consequences of these changes in the procedure are considered in the discussion section.

The Calorie Estimation Task in the present study employed 2 food item lists (see Appendix B), each of which contained 15 different food items. Each food item in each list included a brief description of the type and portion of the food item. The food items on each list were selected through a food item pilot study, and were selected based on criteria outlined by Gino (2008; described below). 
Participants provided an open-ended estimate of how many calories they thought each food item contained (range $=4-701, M=185.20, S D=54.61$ ). Participants' estimates had good internal consistency $(\alpha=.97)$. Following Gino, participants also provided a confidence interval for each of their calorie estimates. That is, participants were instructed to provide a low calorie value and a high calorie value, such that they were $90 \%$ confident that the actual caloric value of the food item fell within that range. For instance, if a participant provided a low value of 100 calories and a high value of 300 calories, they were indicating that they were $90 \%$ confident that the actual caloric value of the food item was no less than 100, and no more than 300 calories. Participants did not receive any feedback regarding the accuracy of their calorie estimates in any of the 4 phases of the Calorie Estimation Task (Gino), but did receive information regarding the actual caloric content of the food items and the accuracy of their estimates in a debriefing that was mailed to them after the study was complete.

Calorie Estimation Task pilot studies. Two pilot studies were conducted to develop and test the Calorie Estimation Task, as well as the full procedure of the study. The first, a food item pilot study, was conducted to select food items to include on the food item lists used in the Calorie Estimation Task (see Appendix B), and also to obtain calorie estimates from a nutritionist that would be used as expert advice that participants could purchase in the Calorie Estimation Task. The food item pilot study involved having people estimate the caloric content of 60 different food items; five WVU undergraduate students, five older adults, and one food expert (a licensed dietician faculty member from the WVU department of Human Nutrition and Foods) completed the food item pilot study. Food items from this pilot study were selected for inclusion in the Calorie Estimation Task in the main study according to two criteria used by Gino (2008). First, food items that people were capable of making a rough calorie estimate for without 
a high probability of accuracy in the pilot study were used in the Calorie Estimation Task in the main study (Gino). Secondly, food items that elicited calorie estimates with approximately equal variances in the pilot study were used in the Calorie Estimation Task in the main study (Gino).

The second pilot study was conducted to test the full procedure of the main study, and to assess whether participants would purchase advice during the Calorie Estimation Task (the procedure for this pilot study was identical to the procedure for the main study). This pilot study involved 4 younger adults (undergraduate students from WVU). During the pilot study, 2 pilot participants purchased advice in both price of advice conditions, and 2 pilot participants purchased advice in a single price of advice condition in the Calorie Estimation Task. Additionally, rates of advice purchasing during the Calorie Estimation Task were monitored for the first 10 younger adult participants who enrolled in the main study. Out of the first 10 younger adult participants, 4 purchased advice in both price of advice conditions in the Calorie Estimation Task, 3 purchased advice in a single condition, and 3 did not purchase any advice. The rates of advice purchasing among participants in the pilot study and the first 10 participants in the main study were similar to those observed by Gino (study $1 ; 2008$ ).

Quality of Advice Scores. Participants who purchased advice responded to a measure of their perceived quality of the advice they received. This measure, adapted from Gino (2008), asked participants to respond to a 7-point likert scale regarding how accurate they perceived the advice they received to be (1="not accurate at all" to 7="very accurate"; actual range =3-7). Additionally, participants who purchased advice responded to the question "How much do you think the person who provided advice knew about calories?" on a 5-point likert scale: (1= "they knew much less about calories than me" to 5= "they knew much more about calories than me"; actual range $=2-5$ ). A Quality of Advice Score was created by taking the mean of the two items 
for each phase that the participant purchased advice in. Thus, a participant could receive a Quality of Advice Score for the small price of advice condition (actual range $=2.50-6, M=4.51$, $S D=.79)$ and/or a Quality of Advice Score for the large price of advice condition (actual range = 3-6, $M=4.45, S D=.81$; see Tables 3 and 4), with higher scores indicating a higher perceived quality of advice.

Estimate Difference Scores. An Estimate Difference Score was calculated for each food item in the small and large price of advice conditions by subtracting the participant's initial calorie estimate (i.e., from either Phase 1 or Phase 3 ) from their final calorie estimate (i.e., from either Phase 2 or Phase 4, respectively). This procedure sometimes produced negative values (for instance, if a participant's initial estimate was 90 and their final estimate was 100, the Estimate Difference Score would have been 90-100=-10). Because the purpose of the Estimate Difference Score was solely to show how many calories a participant's final estimate differed from their initial estimate, the issue of whether the final estimate was larger or smaller than the initial estimate, and thus the sign of the Estimate Difference Score (positive or negative) was irrelevant. Therefore, for ease of interpretation, only absolute values were used (e.g., if a participant's initial investment was 110, and their final estimate was 100, their Estimate Difference Score was 10 - as opposed to -10). This provided a measure of how much the participant's final estimate differed from their initial investment for each food item in a given price of advice condition. A total Estimate Difference Score was calculated for each price of advice condition by summing the Estimate Difference Scores for each food item within each condition. Thus, each participant received a Small Price of Advice Condition Estimate Difference Score (actual range $=81-2716, M=791.22, S D=535.94$ ) and a Large Price of Advice Estimate Difference Score (actual range $=100-2107, M=628.27, S D=385.53$ ); larger 
scores indicated a larger total difference between a participant's initial and final estimates within a given condition.

Weight of Advice Scores. A Weight of Advice (WOA) Score was computed using the formula used by Gino (2008). Specifically, the WOA Score was based on participants' calorie estimates and the advice estimates from each of the 4 phases, and showed whether or not a participant used the advice that they received, and if so, to what degree (Gino; Yaniv, 2004). The WOA Scores were computed as follows: (final estimate - initial estimate) $\div$ (advice estimate initial estimate). A WOA Score was computed for every food item in each calorie estimate condition in which a participant purchased advice.

For instance, in a scenario where a participant made an initial estimate of 600 calories for a given food item in Phase 1, and then received an advice estimate in Phase 2 indicating that the same food item is 300 calories, their final estimate in Phase 2 may have changed to 400 calories, in light of the advice they received. In such a scenario, the WOA Score was: $(400-600) \div(300$ $-600)=.67$, which indicated that the participant changed their final estimate to correspond more closely with the advice estimate they received.

For participants who chose a final estimate that was some value between their initial estimate and the advice estimate, the WOA Score ranged from 0 to 1 . A score of 0 indicated that the advice estimate in no way influenced the participant's final estimate (i.e., the participant did not change their final estimate from their initial estimate). A score of 1 indicated that the advice estimate completely influenced the participant's final estimate (i.e. the participant changed their final estimate to match the advice estimate). A WOA Score of .5 indicated that a participant's final estimate was the average of their initial estimate and the advice estimate. 
The procedure for calculating WOA Scores sometimes produced scores that were illdefined (Gino, 2008). Specifically, cases in which the value estimate in the advice equaled the participant's initial value estimate produced a WOA Score that was a value divided by 0 , which made it impossible to quantify whether or not a participant used the advice (Gino). Furthermore, when a participant's final value estimate was not a value between their initial value estimate and the value estimate in the advice, the WOA Score was greater than 1. For example, if a participant's initial calorie estimate was 100 , the advice value was 150 , and the participant's final estimate was 175 , their WOA Score for that food item would be $(175-100) \div(150-100)=1.5$. Following Gino, cases in which the WOA Score were greater than 1 were recoded to 1 prior to further analyses. In the present study, $31 \%$ of WOA Scores were greater than 1, and thus were recoded to 1 ; there were no instances in which the value estimate in the advice equaled the participant's initial value estimate. The instances of WOA Scores that exceeded 1 were relatively rare in Gino's study (study 1) - 3\% of WOA Scores were greater than 1 in Gino's study, and were thus re-coded.

Additionally, in order for a WOA Score to be computed, a participant must have chosen to purchase advice. Thus, a participant who did not choose to purchase advice for a given food item list did not receive any WOA Scores for the food items from that list. Alternately, a participant who chose to purchase advice in 1 of the 2 price of advice conditions received 1 set of WOA Scores, whereas a participant who chose to purchase advice in both price of advice conditions received 2 sets of WOA Scores. A condition WOA Score was computed by summing the WOA Scores for the food items within each price of advice condition (small price of advice condition actual range $=1.50-15.00, M=8.87, S D=3.61$; large price of advice condition actual range $=2.40-15.00, M=8.17, S D=3.35)$. The Small Price of Advice Condition WOA Scores 
had good internal consistency $(\alpha=.89)$, as did the Large Price of Advice Condition WOA Scores $(\alpha=.85)$.

Calorie Estimate Sunk-Cost Fallacy Scores. Participants who purchased advice in both price of advice conditions received sunk-cost fallacy scores for every analogous pair of food items from each of the food item lists. The sunk-cost fallacy scores were based on comparing the WOA scores from the large and small price of advice conditions; the sunk-cost fallacy was defined as instances when a participant weighted advice more heavily in the large price of advice condition than in the small price of advice condition. Specifically, each time a participant's WOA Score for a given food item in the large price of advice condition exceeded their WOA Score for the analogous food item in the small price of advice condition, the participant received an item sunk-cost fallacy score of " 1 ", indicating that the participant had followed the more expensive advice more closely than the less expensive advice, thus committing the fallacy for those food items. Alternatively, each time a participant's WOA Score for a given food item in the large price of advice condition was equal to or less than their WOA Score for the analogous food item in the small price of advice condition, they received an item sunk-cost fallacy score of “0”, which indicated that the fallacy was not demonstrated. Participants who purchased advice in both price of advice conditions received 15 item sunk-cost fallacy scores; one for each pair of food items. Item sunk-cost fallacy scores were summed to create a Calorie Estimate Sunk-Cost Fallacy Score (actual range $=0-13, M=6.28, S D=3.52 ;$ Klaczynski, 2001; Strough et al., 2008; see Tables 3 and 4). Higher scores indicated more frequent demonstration of the sunk-cost fallacy.

Advice Adherence Scores. In response to the relatively large percentage of ill-defined WOA Scores (i.e., WOA Scores exceeding 1), and the subsequent relatively large percentage of 
Calorie Estimate Sunk-Cost Fallacy Scores that were based on ill-defined WOA Scores, additional measures of advice following and the sunk-cost fallacy based on advice following were developed in the present study. For participants who purchased advice in a given price of advice condition, an Advice Adherence Score was computed for each food item within the condition. Advice Adherence Scores were created by first subtracting the participant's initial calorie estimate (i.e., from either Phase 1 or Phase 3 ) from the advice value, and also subtracting their final calorie estimate (i.e., from either Phase 2 or Phase 4) from the advice value, which created initial and final advice difference scores for each food item. Then, for each food item, the final advice difference score was subtracted from the initial advice difference score, creating an item Advice Adherence Score for each food item. Only the absolute values of the initial and final advice difference scores were used to create the item Advice Adherence Scores (i.e., if a participant's initial estimate was 50 and the advice value was 40 , the initial advice difference score that was used to create the Advice Adherence Score was 10 - as opposed to -10). For instance, if a participant's initial estimate was 120, their final estimate was 140, and the advice value was 150, they would receive an Advice Adherence Score of 20; [(150-120=30), (150$140=10),(30-10=20)]$.

Advice Adherence Scores for each food item represented the degree to which a participant's final estimate adhered to, and/or deviated from, the advice value, relative to the participant's initial estimate. Positive Advice Adherence Scores indicated that the final estimate was closer to the advice value than the initial estimate; the larger the positive value, the closer the final estimate was to the advice value, relative to the initial estimate. Negative Advice Adherence Scores indicated that the final estimate was further away from the advice than the initial estimate; larger negative values (in absolute values) indicated that he final estimate was 
further from the advice value, relative to the initial estimate. For instance, if a participant's initial estimate was 135 , their final estimate was 145 , and the advice value was 150 , the Advice Adherence Score would be 10. Alternately, if the initial estimate was 145, the final estimate was 135, and the advice value was 150, the Advice Adherence Score would be -10 .

Advice Adherence Scores are interpretable in situations in which the final estimate is not a value that is between the initial estimate and the advice value-such scenarios produce WOA Scores that are ill-defined. In such scenarios the meaning of an Advice Adherence Score is the same as when the final estimate is a value that is between the initial estimate and the advice value. Specifically, positive Advice Adherence Scores indicate that the final estimate was closer to the advice value than the initial estimate, while negative scores indicate that the final was further from the advice value than the initial estimate-regardless of whether the final estimate was or was not a value that was between the initial estimate value and the advice value. For instance, if a participant's initial estimate was 165 , their final estimate was 145 , and the advice value was 150, the Advice Adherence Score would be 10 (a positive score indicating that the final estimate value was closer to the advice value than the initial estimate). Alternately, if a participant's initial estimate was 145 , their final was 165 , and the advice was 150 , the Advice Adherence Score would be -10 (a negative score, indicating that the final estimate value was further from the advice value than the initial estimate).

An Advice Adherence Score of 0 indicates that a participant's final estimate neither adhered to, nor deviated from, the advice value. A participant can receive a score of 0 in two ways - by making identical initial and final estimates, or by making a final estimate that is exactly the same number of calories above or below the advice value as the initial estimate (for instance, if the initial estimate was 125 , the advice value was 150 , and the final estimate was 
175). Because the purpose of the Advice Adherence Score is to show whether a participant adhered to advice, a score of 0 is conceptually similar whether the person didn't change their estimate, or whether they did, but the final was exactly the same distance above or below the advice as the initial. Specifically, in both cases, a participant's final estimate is no closer and no further away from the advice than the initial estimate, showing that the participant neither adhered to, nor deviated from, the advice value.

An Advice Adherence Score was created for both the small (actual range $=-47$ to 2660, $M=875.04, S D=630.24$ ) and the large price of advice conditions (actual range $=-160$ to 2067 , $M=672.13, S D=500.01)$ by summing the item Advice Adherence Scores within each condition. Larger scores indicated that a participant's final estimates adhered more closely to advice values in a given condition, relative to their initial estimates.

Advice Adherence Sunk-Cost Fallacy Scores. Item Advice Adherence Scores from the large price of advice condition were compared to the analogous item Advice Adherence Scores from the small price of advice condition to create Advice Adherence Sunk Cost-Fallacy Scores. Specifically, participants who purchased advice in both price of advice conditions received Advice Adherence Sunk-Cost Fallacy Scores for every analogous pair of food items from each of the food item lists. The sunk-cost fallacy was defined as instances when a participant's Advice Adherence Score for a given food item was larger in the large price of advice condition than for the analogous food item in the small price of advice condition, in which case the participant received an Advice Adherence Sunk-Cost Fallacy Score of "1". An Advice Adherence SunkCost Fallacy Score of "1" indicated that a participant's final estimate adhered more closely to the advice value relative to their initial estimate in the large price of advice condition than in the small price of advice condition, thus demonstrating the fallacy. Alternatively, each time a 
participant's Advice Adherence Score for a given food item in the large price of advice condition was equal to or less than their Advice Adherence Score for the analogous food item in the small price of advice condition, they received an Advice Adherence Sunk-Cost Fallacy Score of "0", indicating that the fallacy was not demonstrated for that pair of food items.

Participants who purchased advice in both price of advice conditions received 15 Advice Adherence Sunk-Cost Fallacy Scores; one for each pair of food items. Advice Adherence SunkCost Fallacy Scores were summed to create a Total Advice Adherence Sunk-Cost Fallacy Score (actual range $=1-13, M=5.94, S D=3.27$; see Tables 3 and 4 ). Higher scores indicated more frequent demonstration of the sunk-cost fallacy.

Confidence Interval Scores. A Confidence Interval Score for each of the participants' calorie estimates in all 4 phases of the calorie estimate task was computed. The Confidence Interval Score was the difference between the upper and lower calorie values that participants selected for their confidence interval for the calorie value of each food item. Thus, larger Confidence Interval Scores suggest that a participant was less confident in the accuracy of a given calorie estimate. A Pre-Advice Confidence Interval Score was created for both Phase 1 and Phase 3 by taking the mean of the Confidence Interval Scores from Phases 1 and 3; these means were then reverse-scored so that larger scores represented greater confidence (Phase 1 actual range $=92.53-486, M=375.96, S D=74.29 ;$ Phase 2 actual range $=172.67-484.27, M=378.42$ , $S D=70.96$ ). Participants' Confidence Interval Scores for Phase 1 had good internal consistency $(\alpha=.94)$, as did the Confidence Interval Scores for Phase $3(\alpha=.95)$. Additionally, a Mean Pre-Advice Confidence Interval Score was created by taking the mean of all Confidence Interval Scores from Phases 1 and 3; these means were then reverse-scored so that larger scores 
represented greater confidence (actual range $=237-483.33, M=383.81, S D=55.78$; see Tables 3 and 4).

Accuracy Scores. Item pre-advice estimate Accuracy Scores were created by coding each accurate calorie estimate (i.e. an estimate that was within $10 \%$ of the actual caloric value of the food item) from Phase 1 and Phase 3 as a "1". Participants' item pre-advice estimate Accuracy Scores had adequate internal consistency $(\alpha=.73)$. The Pre-Advice Calorie Estimate Accuracy Score was created by summing the item pre-advice estimate Accuracy Scores across Phases 1 and 3 (actual range $=0-18, M=6.87, S D=4.15$; see Tables 3 and 4); higher scores indicated more accurate estimates in Phases 1 and 3.

Final Winnings Score. A score to represent the final amount of money that participants received from accurate calorie estimates was created. The Final Winnings Score was simply the total amount, in dollars and cents, that each participant won by making accurate estimates during the Calorie Estimation Task (actual range $=0-10.00, M=5.18, S D=2.55$ ).

Loss Aversion: Delay Discounting Task. All participants completed a Delay Discounting Loss Aversion Task adapted from Green and colleagues (1994; see Appendix C). This task involved 2 conditions - a gain and a loss condition. In each condition, participants made a series of choices between gaining/losing a fixed-amount of hypothetical money following a hypothetical time delay, and gaining/losing a hypothetical amount of money immediately. For instance, in the gain condition, a participant may have been asked whether they would prefer to gain $\$ 10$ immediately or $\$ 1000$ after a time delay of 1 week. Conversely, in the loss condition, a participant may have been asked whether they would prefer to lose $\$ 10$ immediately or $\$ 1000$ after a time delay of 1 week. 
Both the gain and the loss condition consisted of 16 trials, each of which involved making a choice between an immediate gain/loss, and a gain/loss at one of eight different time delays. The eight different time delays were: 1 week, 1 month, 6 months, 1 year, 3 years, 5 years, 10 years, and 25 years (Green et al., 1994). For each of the eight time delays, the immediate gain/loss values was presented twice; once in ascending order, and once in descending order (order of presentation was counterbalanced across participants). Moreover, in both the gain and loss condition, each trial consisted of a choice between one of 30 different immediate gain/loss values and the fixed-value delayed gain/loss. The 30 different immediate gain/loss values ranged from $0.1 \%$ to $100 \%$ of the fixed-value delayed gain/loss value (Green et al.; see Appendix C). The fixed-value delayed gain/loss was $\$ 1000$ in all trials of both conditions.

All hypothetical monetary values appeared as buttons on a computer screen that participants could click using a mouse. The buttons were presented so that the button on the participants' right had a fixed dollar amount that the participant would hypothetically receive after a time delay. The buttons on the participants' left had monetary values that the participant would hypothetically receive immediately. Participants made a series of choices between the fixed, delayed amount on their right (e.g. $\$ 1,000$ ), and the immediately available value on their left, which varied in either ascending or descending order from trial to trial (e.g. from $\$ 1$ to $\$ 1,000$ or from $\$ 1,000$ to $\$ 1$; Green et al., 1994).

The differences between the methods used in Green and colleagues' (1994) study and this study are that Green and colleagues used two fixed-amount gains of $\$ 1000$ and $\$ 10,000$, whereas this study used a fixed-amount gain of $\$ 1000$. Green and colleagues utilized two fixed-amount gains in order to assess how people discount delayed gains of different values - the authors found age differences in delay-discounting of both of their fixed-amount gain values. Because 
this study is not intended to assess the parameters of delay-discounting, only one delayed fixed amount gain/loss value (\$1000) was used. Additionally, this study included a delay discounting of losses condition (Estle, Green, Myerson, \& Holt, 2006), whereas Green and colleagues'study did not; this was done in order to assess loss aversion in the study (see Appendix C).

Delay Discounting Loss Aversion Score. Loss aversion scores for the delay discounting task were based on an area under the curve measure of discounting adapted from Myerson and colleagues (2001). Specifically, two curves were plotted for each participant; one based on participants' preferences for immediate versus delayed gains as a function of delay, and the other based on immediate versus delayed losses as a function of delay. Each curve was plotted using normalized subjective monetary values and normalized delay values, and an area under each curve was calculated (Myerson et al.).

Specifically, a subjective monetary value was calculated for each delay value in both the gains and the losses conditions for each participant. Subjective monetary values were defined as the amount of an immediate monetary gain or loss that the participant judged to be equal to the delayed gain or loss - that is, the monetary value at which a participant was indifferent between the immediate gain or loss and the delayed gain or loss. Each subjective value for the gains condition was the mean of the monetary value at which the participant switched from the larger delayed gain to the smaller immediate gain when the immediate gains were presented in ascending order, and the monetary value at which the participant switched from the smaller immediate gain to the larger delayed gain when the immediate gains were presented in descending order (Green et al., 1994). Similarly, each subjective monetary value for the losses condition was the mean of the monetary value at which the participant switched from a larger delayed loss to a smaller immediate loss when the immediate losses was presented in descending 
order, and the monetary value at which the participant switched from the smaller immediate loss to the larger delayed loss when immediate losses were presented in an ascending order. These subjective monetary values were normalized by dividing by the fixed delayed monetary gain/loss (i.e., by dividing by $\$ 1,000)$. Delay values were also normalized by expressing delays as a proportion of the maximum delay (i.e., a proportion of 25 years; Myerson et al., 2001).

Normalized subjective monetary values from both the gains and losses condition were plotted as a function of normalized delay values. Specifically, the normalized subjective monetary values were used as $y$ coordinates, and the normalized delay values were used as $x$ coordinates to create data points from which to plot the gains curve and the losses curve (Myerson et al.; see Figure 5 for an example illustration of graphed normalized subjective monetary values plotted as a function of normalized delay values). Then, a vertical line was drawn from each data point in both the gains and the losses curves downward to the $x$ axis, which subdivided the area under each respective curve into a series of trapezoids. The area of each trapezoid was then calculated using the formula $\left(x_{2}-x_{1}\right)\left[\left(y_{1}+y_{2}\right) / 2\right]$, where $x_{1}$ and $x_{2}$ represented sequential normalized delays and $y_{1}$ and $y_{2}$ represented each normalized subjective monetary value associated with $x_{1}$ and $x_{2}$. The total area under each curve (AUC) was then calculated by summing the areas of each trapezoid under each curve (Myerson et al.). Thus, an AUC score was calculated for the gains (actual range $=.01-.91, M=.42, S D=.21$ ) and the losses (actual range $=.01-.73, M=.29, S D=.22$ ) condition, with smaller AUC scores indicating a greater degree of discounting. The AUC gains score was subtracted from AUC losses score-this value served as the Delay Discounting Loss Aversion Score (actual range = -.65 to $.35 M=-.12$, $S D=.21)$. Larger positive Delay Discounting Loss Aversion Score values indicated greater loss aversion (i.e., a greater preference for smaller immediate gains over larger delayed gains relative 
to a greater preference for larger delayed losses over smaller immediate losses—or a greater discounting of delayed gains compared to delayed losses. Larger negative (in absolute values) Delay Discounting Loss Aversion Scores indicated less aversion to losses (i.e., a greater preference for larger delayed gains over smaller immediate gains relative to a greater preference for smaller immediate losses over larger delayed losses — or lesser discounting of delayed gains compared to delayed losses; See Figure 5 for an example illustration of graphed normalized subjective values used to calculate AUC Gains and Losses Scores and the Delay Discounting Loss Aversion Score)

Reversed AUC Gains and Losses Scores. For ease of interpretation, after the Delay Discounting Loss Aversion Score was created, the AUC gains and losses scores were each reversed so that greater values indicated greater discounting of gains and losses, respectively. This created a Reversed AUC Gains Score (actual range = .09-.99, $M=.58, S D=.20$ ), and a Reversed AUC Losses Score (actual range = .27-.99, $M=.71, S D=.27$; see Tables 3 and 4). These scores were used to assess discounting of delayed gains and losses (but not loss aversion).

Loss Aversion: Tradeoff Task. All participants completed a Tradeoff Loss Aversion Task adapted from Fennema and Van Assen (1999). Participants were presented with a series of pairs of buttons on the computer screen, each of which included a diagram outlining a scenario that involved a possible monetary gain, and a possible monetary loss, each of which had a $50 \%$ chance of occurring (see Appendix D). Participants chose between the scenario on the button on their left, which changed from trial to trial, and the scenario on the button on their right, which remained the same; the $50 \%$ chance of a gain or loss on each button remained constant. 
For instance, a participant may have viewed a scenario button on their left which indicated a $50 \%$ chance of gaining $\$ 2000.00$, and a $50 \%$ chance of losing $\$ 25.00$, and a scenario button on their right which indicated a 50\% chance of gaining $\$ 1500.00$ and a $50 \%$ chance of losing $\$ 25.00$. A rational participant would have chosen the scenario button on their left in this instance, since there was an equal probability of gains and losses on each scenario button, the amount of possible loss was the same, but the amount of possible gain was larger on the scenario button on the left (by \$500.00). In subsequent trials, the loss value on the scenario button on the left increased by $\$ 5$ while all other values remained the same. Because the loss value on the scenario button on the left increased, it became progressively less desirable to choose the scenario button on the left_even though the gain value on the scenario button on the left was always larger than the gain value on the scenario button on the right.

As the loss value on the scenario button on the left increased from trial to trial, the participant would eventually view the potential loss on the scenario button on the left as outweighing the potential gain on the scenario button on the left (even though this potential gain was larger than the potential gain on the scenario button to the right). When a participant switched preference from the scenario button on the left to the scenario button on the right (i.e., when they reached their "indifference point"), the session ended, and a new session began with new monetary values.

In each subsequent session, the possible gain values remained the same on each scenario button. However, the loss values on the first trial of each session were different than in previous sessions (see Appendix D). The Tradeoff Loss Aversion Task in this study was carried out for four sessions (Fennema \& Van Assen, 1999). However, unlike this study, Fennema and Van Assen used a procedure in which the loss values in each session were the value of the 
participant's "indifference point" from the previous session—-thus, the loss values in all sessions except for the first were different across participants. However, this study used the same loss values on the first trial of each session for all participants to avoid confounding participants' "indifference points" with the loss value on the first trial of each session. Additionally, Fennema and Van Assen varied both the gains and the losses values, and assessed certainty equivalence values, and the shape of the utility function of losses. Moreover, Fennema and Van Assen used different probability values for the gain and loss values. The purpose of the Tradeoff Loss Aversion Task in this study was solely to assess loss aversion, which can be measured based on participants' "indifference points" when loss values are varied. Therefore, this study did not include varying gains values, certainty equivalence values, utility functions, or differing probabilities for gain and loss values as Fennema and Van Assen's study did (see Appendix D).

Tradeoff Loss Aversion Task Score. A loss aversion score for the tradeoff task was based on participants' "indifference points" in each of the 4 sessions of the task. Participants who switched preferences from the scenario button on the left (with the larger gain value and the varying loss value) to the scenario button on the right (with the smaller gain value and the fixed loss value) at earlier trials within a session had a smaller "indifference point" value for a given session than participants who switched preferences at later trials. Thus, a larger "indifference point" value within a session indicated that a participant was willing to risk a greater loss than a participant with a smaller "indifference point" value. For example, if a participant's "indifference point" was $\$ 150.00$, it would indicate that $\$ 150.00$ was the largest potential loss they were willing to tolerate before switching to the scenario button on the right, which had a smaller potential gain and loss than the scenario button on the left. Alternatively, if a participant's "indifference point" was $\$ 50.00$, it would indicate that $\$ 50.00$ was the largest potential loss they 
were willing to tolerate-in this example, the second participant is willing to tolerate a smaller potential loss than the first participant, and thus is more loss averse, Participants' "indifference point" values across the 4 sessions of the tradeoff task had good internal consistency $(\alpha=.94)$. The Tradeoff Loss Aversion Task Score was calculated by taking the mean of participants' “indifference point" values across all 4 sessions of the task, and reverse-scoring each mean so that larger scores indicate greater loss aversion (actual range $=0-495, M=383.12, S D=140.75$; see Tables 3 and 4).

The Subjective Numeracy Scale. Participants completed the Subjective Numeracy Scale (SNS; Fagerlin et al., 2007; Appendix E), an 8-item self-report questionnaire of numerical ability. The SNS consisted of items such as "How good are you at calculating a 15\% tip?"; participants responded on a 6-point likert scale (1= "not at all good", 6= "extremely good"). Prior studies have shown that the SNS has good convergent validity with a variety of measures of statistics comprehension and quantitative ability (Zikmund-Fisher, Smith, Ubel, \& Fagerlin, 2007). The SNS had good internal consistency in this study $(\alpha=.79)$. The SNS Scores were calculated by computing the mean of the scores from the 8 items (actual range $=1-6, M=3.89$, $S D=.95$; see Tables 3 and 4); higher scores indicated better perceived numerical ability.

\section{The weight control subscale of the Food Choice Questionnaire. Participants} completed the weight control subscale of the Food Choice Questionnaire (FCQ; Steptoe et al., 1995; see Appendix F). This subscale consisted of 3 items which asked about participant's dietary habits (e.g.; "It is important to me that the food I eat on a typical day is low in calories"). Participants responded on a 4-point scale to indicate how important each item was to them (e.g.; "1=not at all important", " $2=$ a little important", " $3=$ moderately important", " $4=$ =very important"). Prior research has shown that the weight control subscale correlates with measures of healthy 
diet and has good test-retest reliability ( $r=.81$; Steptoe et al.). The weight control subscale had good internal consistency in this study $(\alpha=.81)$. The FCQ Score was computed by taking the mean of the 3 items (actual range $=1-4, M=2.75, S D=.80$; see Tables 3 and 4); higher scores indicated better attention to weight control.

The Health Habits Questionnaire. As an exploratory measure, participants completed a 22-item Health Habits Questionnaire (HHQ), which was developed specifically for this study (see Appendix F). The questionnaire consisted of items concerning participants' nutritional and health habits, and their perceptions of their own health compared to other people their age. Specifically, items 1 through 11 asked participants about their health-related behaviors (e.g.; “How concerned are you with checking nutrition information on food labels?"). Participants responded on a 4-point scale (" $1=$ not at all concerned", " $2=$ a little concerned", " $3=$ moderately concerned", "4=very concerned"). Items 12 through 17 asked participants about their perceptions of their own health (e.g.; "Compared with most other people your age, would you say your health is...). Participants responded on a 3 -point scale (" $1=$ better", " $2,=$ the same", " $3=$ not as good"). Items 18 through 22 asked participants about their prior health-related behaviors (e.g. “Have you ever paid money to join a gym or fitness club?"). Participants responded on a dichotomous 2-point scale (i.e. " $1=$ yes", " $2=$ no"). The HHQ had good internal consistency ( $\alpha=$ .85 ). The HHQ Score was computed by taking the mean of the 22 items (actual range $=1.32$ 2.86, $M=2.17, S D=.35$; see Tables 3 and 4); higher scores indicated better health habits.

The Sunk Cost Vignette Questionnaire. Participants completed the Sunk Cost Vignette Questionnaire, which consisted of 2 vignette pairs, separated by 2 "filler" vignette pairs ( 8 different vignettes, from Strough et al. 2008; as adapted from Frisch, 1993). One member of each pair of vignettes contained information about a prior investment; the other member of each pair 
was a no-investment analogue and either did not contain information about a prior investment, or contained information about a prior investment that was smaller than in the investment analogue. Vignettes either involved the presence or absence a time investment (e.g.; "You have been working on a project related to one of your hobbies for five years...”), or a monetary investment (e.g.; "You paid $\$ 10.95$ to see a movie on pay TV..."). After reading each vignette, participants responded on a 5-point scale to indicate the amount of future investment that they would commit (e.g., stop watching entirely, watch for 10 more min, watch for 20 more min, watch for 30 more min, watch until the end). Presentation of each vignette from a given pair was separated by 2 other vignettes that were not part of the pair, and order of presentation of vignettes was counterbalanced. The Sunk Cost Vignette Questionnaire had adequate internal consistency in this study $(\alpha=.70)$.

Sunk-Cost Fallacy Vignette Scores. Sunk-cost fallacy scores were computed by comparing each participant's decision for the investment vignette to their decision for the noinvestment analogue vignette (Klaczynski, 2001). This was done using data from two vignette pairs used in prior studies of age differences in the sunk-cost fallacy (Strough et al., 2008; 2011b) which described scenarios where the investment was in an activity (watching a movie or pursuing a hobby). Specifically, if a participant indicated that they would commit a greater future investment in response to an investment vignette than they did in response to the no-investment analogue vignette, a score of 1 was assigned to indicate that the sunk-cost fallacy occurred for that pair of vignettes; otherwise the score was 0 . Scores from the two pairs of vignettes were summed to create a Sunk-Cost Fallacy Vignette Score (actual range $=0-2, M=.84, S D=.65$; higher scores indicate more frequent demonstration of the sunk-cost fallacy; see Tables 3 and 4). 
Sunk-Cost Fallacy Vignette Overinvestment Scores. Overinvestment scores were computed for the vignette questionnaire (Strough et al., 2008) for participants who demonstrated the sunk-cost fallacy on a given pair of vignettes by taking the difference between the amount the participant invested when responding to the investment vignette and the amount they invested in response to the no-investment analogue. This created overinvestment scores for each of the two pairs of vignettes used in this study. Participants who invested less in response to the investment vignette than the no-investment vignette from a given pair did not receive an overinvestment score for that pair of vignettes (i.e.; participants who underinvested-this occurred for $5 \%$ of the The Sunk Cost Vignette Questionnaire data; no participants underinvested on both of the vignette pairs). The Sunk-Cost Fallacy Vignette Overinvestment Score was then computed for each participant by summing their overinvestment scores from each of the two pairs of vignettes (actual range $=0-6, M=1.90, S D=1.64$; higher scores indicated a greater degree of overinvestment; see Tables 3 and 4).

Normatively Correct Scores. For each vignette pair, a Normatively Correct Score was computed (Klaczynski, 2001). Specifically, if a participant indicated the same amount of continued investment in response to both vignettes from a given pair, they received a score of 1 , indicating that they had made the normatively correct decision for that pair of vignettes (i.e., the participant responded identically to both vignettes from a given pair, regardless of the presence or absence of a sunk cost). When a participant responded differently across vignettes from a given pair (i.e., if they indicated a greater or lesser continued investment in response to one vignette in comparison to its analogue), a score of 0 was assigned, indicating that the participant did not make the normatively correct decision in response to that pair of vignettes. Normatively correct responses were summed across the two vignette pairs to create a Normatively Correct 
Score (actual range $=0-2, M=.67, S D=.62$; higher scores indicate more frequent demonstration of the normatively correct decision; see Tables 3 and 4).

The Spendthrift-Tightwad Scale. The Spendthrift-Tightwad Scale (STS; Rick et al., 2008; Appendix H) was used to measure "pain of paying" (Rick et al., p. 767), or the degree to which participants perceived spending money as difficult. The STS consisted of 4 items - the first 3 of which asked participants to rate how well statements pertaining to spending habits described them; participants responded on an 11-point likert scale (item 1) and a 5-point likert scale (items 2 and 3). The fourth item on the STS was a vignette that described two characters, one of whom had difficulty controlling their spending (i.e. a "spendthrift"), the other of whom had difficulty spending money (i.e. a "tightwad"). Participants rated which character they were most similar to on a 5-point likert scale. Prior research has shown that the STS relates to how much money people save, as well as their credit card debt, suggesting the validity of the scale (Rick et al.). The STS has adequate internal consistency in this study ( $\alpha=.67)$. The STS Score was created by summing participants' responses to each of the 4 items (actual range $=4-25, M=$ 14.96, $S D=3.87$; see Tables 3 and 4); higher scores indicated greater difficulty controlling spending.

The Future Time Perspective Scale. Future time perspective was assessed using Carstensen and Lang's (1996; see Appendix I) 10-item scale (the FTPS), which included items such as (e.g., "There is plenty of time in my life to make new plans."). Participants responded by indicating their agreement with each item on a 7 -point scale $(1=$ very untrue, $7=$ very true $)$. Prior research has shown an inverse relation between age and future time perspective (FTP), suggesting that older people have more limited FTP than younger adults (Lang \& Carstensn, 2002). The FTPS had good internal consistency in this study $(\alpha=.91)$. Future Time Perspective 
Scores were computed by taking the mean of participants' responses to each item (actual range $=$ 1.40-7, $M=4.66, S D=1.32$; see Tables 3 and 4); higher scores indicated more expansive futuretime perspective.

Demographics. All participants completed a self-report demographics form, which included self-reports of age, sex ethnicity, and race (see Appendix J). The demographics form also included a measure of participants' ability to pay their bills; participants were asked "How much difficulty to you have paying your bills?", and responded on a 4-point scale (1 = "a great deal of difficulty", 4 = "no difficulty"); scores were reversed to create a Difficulty Paying Bills Score; higher scores indicated greater difficulty paying bills (actual range $=0-4 ; M=.85, S D=$ $.97)$.

\section{Results}

\section{Data Screening}

Missing cases. There were a total of 4 missing cases (i.e., items from the questionnaire that didn't receive a response) on the weight control subscale of the Food Choice Questionnaire (FCQ; Steptoe et al., 1995); 1 missing case from the younger adult data (.6\% missing), and 3 missing cases from the older adult data (3\% missing). There were a total of 8 missing cases on the Health Habits Questionnaire (HHQ); 1 missing case from the younger adult data (.09\% missing) and 7 missing cases from the older adult data (.7\% missing). There were a total of 9 missing cases (2\% missing) on the Future Time Perspective Scale (FTPS; Carstensen \& Lang, 1996) from the older adult data. Data sets consisting of 5\% or fewer missing cases generally present minimal problems and the missing cases from such data sets can be addressed with a variety of methods (Tabachnick \& Fidell, 2007). Missing cases from a particular item in the data 
set for the present study were substituted using the mean of that item for the respective age group (Tabachnick \& Fidell, 2007; Widaman, 2005).

Outliers. Univariate outliers were identified using box plots separately for each age group. Specifically, Boxplots provided a graphic depiction of outliers, and depicted values that were more than 1.5 box lengths from either the upper or lower edge of the box (i.e., more than 1.5 times larger or smaller than the interquartile range) as outliers (Field, 2009; Grace \& Sawilowski, 2009). The use of boxplots, which utilize the interquartile range, tends to be more effective in identifying outliers than basing outlier identification on standard deviations, since the standard deviation can be inflated by outliers (Grace \& Sawilowski). Among younger adults, 158 cases $(5 \%)$ of the Calorie Estimation Task data were outliers, among older adults, 357 cases (12\%) of the Calorie Estimation Task were outliers. For younger adults, 322 cases (5\%) of the confidence interval data were outliers, whereas 626 cases $(10 \%)$ of the confidence interval data were outliers for older adults. Among younger adults, 29 cases (14\%) were outliers on the Tradeoff Loss Aversion Task Score. Among older adults, 1 case (4\%) of the Delay Discounting Loss Aversion Score was an outlier. Outliers from each variable were recoded to be one point above the most extreme value that was not an outlier (Grace \& Sawilowsky).

Nonsystematic delay discounting data. Nonsystematic delay discounting is defined by a violation of 1 of 2 criteria; either an indifference point that is $20 \%$ or more greater than the indifference point at the previous delay (criterion 1), or an indifference point at the last delay that is not at least $10 \%$ less than the larger later reward (criterion 2; Johnson \& Bickel, 2008). For instance, if a participant's indifference point at the 1 month delay was $\$ 200$, and their indifference point at the 1 week delay (the immediately preceding delay) was $\$ 100$, they would have violated criterion 1 (Johnson \& Bickel). Also for instance, if a participant's indifference 
point at the 25 year delay (the last delay) was $\$ 950$, a violation of criterion 2 would have occurred because the larger later reward value is $\$ 1000$ (Johnson \& Bickel). Participants who violated criterion 2 were not included in the analyses in this study (Johnson \& Bickel); 18 (36\%) of the younger adults and $23(46 \%)$ of the older adults violated criterion 2. More specifically, 12 $(24 \%)$ of the younger adults violated criterion 2 in the gains condition of the delay discounting task, while 7 (14\%) violated criterion 2 in the losses condition. Likewise, 17 (34\%) of the older adults violated criterion 2 in the gains condition, while $14(28 \%)$ violated criterion 2 in the losses condition. Participants who violated criterion 1 more than once were not included because multiple violations of criterion 1 made an unambiguous interpretation of data impossible; 17 (34\%) of the younger adults and $14(28 \%)$ of the older adults violated criterion 1 more than once. More specifically, among younger adults, 7 (14\%) violated criterion 1 more than once in the gains condition, while $11(22 \%)$ violated criterion 1 more than once in the losses condition. Among older adults, $4(8 \%)$ violated criterion 1 more than once in the gains condition, while 12 (24\%) violated criterion 1 more than once in the losses condition. A total of $27(54 \%)$ of the younger adults and 27 (54\%) of the older adults either violated criterion 1 more than once, violated criterion 2, or both, and thus were excluded from further analyses.

Indifference points that violated criterion 1 were re-coded to be the mean of the indifference points at the preceding and succeeding delays, but only for participants who did not violate criterion 1 more than once $(10$, or $20 \%$ of the younger adults and 6 , or $12 \%$ of the older adults violated criterion 1 once). A total of $13(26 \%)$ of the younger adults did not violate either criteria, a total of 17 (34\%) of the older adults did not violate either criteria. Thus, 23 younger adults (46\% of the younger adult sample) and 23 older adults (46\% of the older adult sample) were included in analyses involving delay discounting. 
Normality. Each variable was assessed for normality by computing $z$-scores for skew and kurtosis independently for each age group. The Tradeoff Loss Aversion Task Score exhibited significant negative skew for younger adults $(z=-3.11, p<.01)$ and for older adults $(z$ $=-1.99, p<.05)$. Given the significant skew, and the shape of its distribution, a log transformation was conducted on the Tradeoff Loss Aversion Task Score (Tabachnick \& Fidell, 2007). The log-transformed Tradeoff Loss Aversion Task Score did not exhibit significant skew for either age group. All analyses reported below involving the Tradeoff Loss Aversion Task Score were conducted using both the log-transformed and the untransformed scores; results of all analyses were the same in terms of direction and significance-for ease of interpretation, only results using the untransformed scores are reported below.

Assumption of homogeneity of variances. Non-significant Levene's tests $(p>.05)$ indicated that the variances of the two age groups were not significantly different on the PreAdvice Calorie Estimate Accuracy Score, the Pre-Advice Confidence Interval Scores (for Phases 1 and 2), the Delay Discounting Loss Aversion Score, the Sunk-Cost Fallacy Vignette Score, the Sunk-Cost Fallacy Vignette Overinvestment Score, the Health Habits Questionnaire (HHQ) Score, the Subjective Numeracy Scale (SNS) Score, the Future Time Perspective (FTP) Score, or the weight control subscale of the Food Choice Questionnaire (FCQ) Score. Significant Levene's tests indicated that the variances of the two age groups were significantly different on the Mean Pre-Advice Confidence Interval Score; $F(1,98)=42.70, p<.001$, the Sunk-Cost Fallacy Vignette Normatively Correct Score; $F(1,98)=11.75, p<.01$; the log-transformed Tradeoff Loss Aversion Task Score; $F(1,98)=5.56, p=.02$, the Sunk-Cost Fallacy Vignette Activity Score; $F(1,98)=8.43, p=.005$, and on the Spendthrift-Tightwad Scale $(\mathrm{STS})$ Score; $F(1,98)=$ $12.84, p=.01$. 
As a follow-up, a variance ratio (i.e. Hartley's $F_{\max }$; Field, 2009) was calculated for each variable that had a significant Levene's test. The group variances of the Mean Pre-Advice Confidence Interval Score had a variance ratio of $F_{\max }=2.05$. The group variances of the Normatively Correct Score had a variance ratio of $F_{\max }=2.15$. The group variances of the logtransformed Tradeoff Loss Aversion Task Score had a variance ratio of $F_{\max }=3.64$. The group variances of the Sunk-Cost Fallacy Vignette Score had a variance ratio of $F_{\max }=1.28$. The group variances of the STS Score had a variance ratio of $F_{\max }=2.06$. Field notes that with larger sample sizes, small group variance differences will produce significant Levene's tests, and that when group sizes are approximately 50 people, a variance ratio of 3 or less is acceptable. Because the variance ratio of the log-transformed Tradeoff Loss Aversion Task Score was greater than 3, a non-parametric independent-samples Mann-Whitney U test was conducted as a follow-up to the MANOVA used to address Research Question 3 (Field, 2009). The results of this analysis were the same as those of the MANOVA in terms of direction and significance; for ease of interpretation, only the results of the MANOVA are reported below.

Order effects and completion time. The orders of presentation of the price of advice conditions (large presented first vs. small first), the food item lists from the Calorie Estimation Task (list 1 presented first vs. list 2 first), the conditions of the Delay Discounting Loss Aversion Task (gains presented first vs. losses first), and the Sunk-Cost Fallacy Vignettes (investment analogue presented first vs. no-investment analogue first) were counterbalanced for half of the participants. Specifically, the order of presentation was reversed after data had been collected from the first 25 participants within each age group. Order effects were assessed with independent samples $t$ tests. Neither younger or older adults differed in the number of times they purchased advice, their Pre-Advice Confidence Interval Scores (for either Phase 1 or Phase 3), 
Mean Pre-Advice Confidence Interval Scores, Pre-Advice Calorie Estimate Accuracy Scores, Calorie Estimate Sunk-Cost Fallacy Scores, or Total Advice Adherence Sunk-Cost Fallacy Scores as a function of order of presentation of price of advice condition or food item list ( $p>$ .05). Neither younger, nor older adults differed in their Delay Discounting Loss Aversion Task Scores as a function of order of presentation of condition (gains first, losses first; $p>.05$ ). Additionally, neither younger nor older adults differed in their Sunk-Cost Fallacy Vignette Score or their Sunk-Cost Fallacy Vignette Overinvestment Score as a function of order of presentation of the vignettes $(p>.05)$.

Time to complete this study was measured by the computer program beginning from the time participants began reading the initial instructions on the computer screen until they clicked a "Finish" button at the end of the protocol. Older adults took significantly longer $(M=65.32$ minutes, $S D=23.52)$ than younger adults $(M=38.88$ minutes, $S D=12.39)$ to complete the study; $t(98)=-7.56, p<.001, r=.61$. Time to complete the study did not vary significantly as a function of order of presentation of conditions for either younger or older adults $(p>.05)$.

\section{Rates of Advice Purchasing}

Based on Gino (2008), it was anticipated that half of research participants would purchase advice in both of the 2 phases in which advice was available. However, out of 50 younger adults, 16 purchased advice in at least one of the conditions in which it was available, 6 purchased advice in both conditions, and 34 did not purchase advice. Significantly more older adults (72\%) purchased advice than younger adults (32\%) in one or both of the phases in which it was available (i.e., purchased advice in at least one condition); $\chi^{2}(1)=16.03, p<.001$. Similarly, significantly more older adults (72\%) purchased advice than younger adults (28\%) in 
the small price of advice condition; $\chi^{2}(1)=19.36, p<.001$. Likewise, significantly more older adults $(52 \%)$ purchased advice than younger adults (16\%) in the large price of advice condition; $\chi^{2}(1)=14.44, p<.001$. There were no significant sex differences in advice purchasing in either price of advice condition, either when each age group was analyzed independently, or when age groups were combined $(p>.05)$. Because the number of younger adults who purchased advice in both phases was insufficient to address several of the research questions and hypotheses, several revised and exploratory research questions and hypotheses were addressed. Furthermore, some research questions were addressed using only data from older adults. Research questions that were revised or added as exploratory questions appear in italics below.

\section{Primary Analyses}

\section{RQs 1, 2, \& 3: Age differences in the sunk-cost fallacy, normatively correct} decisions, overinvestment, and loss aversion. Research questions 1, 2, and 3 (Are there age differences between younger and older adults in how often they demonstrate the sunk-cost fallacy and make the normatively correct decisions when responding to the self-report measure of the sunk-cost fallacy? Are there age differences in degree of overinvestment on the self-report measure of the sunk-cost fallacy? Are there age differences in loss aversion?-respectively) were addressed using a multivariate analysis of variance (MANOVA) and independent-samples $t$ tests. The MANOVA is less prone to Type 1 error than multiple univariate $t$-tests or ANOVAs. However, the MANOVA is more difficult to interpret than univariate tests, and may be prone to low power when intercorrelations of dependent variables are high (.60 and above; Tabachnick $\&$ Fidell, 2007). The MANOVA in this study included age group (younger, older adults) as the between-subjects factor, and Sunk-Cost Fallacy Vignette Score, Sunk-Cost Fallacy Vignette Overinvestment Score, Normatively Correct Score, and the Tradeoff Loss Aversion Task Score 
as the dependent variables; the only intercorrelation between dependent variables that exceeded .60 was the relation between Sunk-Cost Fallacy Vignette Score and Sunk-Cost Fallacy Vignette Overinvestment Score (see Table 5). However, because of the significant results reported below, low power resulting from high intercorrelations between dependent variables did not appear to be an issue. Because the Delay Discounting Loss Aversion Task Score was based on fewer participants than the other scores due to the removal of nonsystematic delay discounting data, this variable was assessed using an independent-samples $t$-test. The MANOVA was conducted with Difficulty Paying Bills Score, income, and sex as covariates; all results were the same in terms of direction and significance. The results of the MANOVA reported below are based on analyses without covariates.

Results of the MANOVA showed that age group had a significant effect on the linear combination of dependent variables; $V(3,96)=.23, F(3,96)=9.56, p<.001, \eta_{p}^{2}=.23$. Planned simple comparisons showed that-systematically replicating (Sidman, 1960) prior studies (i.e., Strough et al., 2008; 2011b) — older adults demonstrated the sunk-cost fallacy less often $(M=.66$, $S D=.66)$ than younger adults $(M=1.02, S D=.59) ; F(1,98)=3.24, p=.005, \eta_{p}^{2}=.08$. Also replicating the findings of Strough and colleagues (2008; 2011b), older adults made the normatively correct decision more often $(M=.80, S D=.67)$ than younger adults $(M=.54, S D=$ $.54) ; F(1,98)=4.55, p=.03, \eta_{p}^{2}=.04$ (see Figure 1), and older adults who demonstrated the sunk-cost fallacy overinvested less $(M=1.14, S D=1.24)$ than younger adults who demonstrated the fallacy $(M=2.66, S D=1.64) ; F(1,98)=27.31, p<.001, \eta_{p}^{2}=.22$ (see Figure 1$)$. Thus, Hypotheses 1 and 2 were supported; older adults demonstrated the sunk-cost fallacy less frequently, and made the normatively correct decision more frequently than younger adults, and 
older adults who demonstrated the sunk-cost fallacy overinvested less than younger adults who demonstrated the fallacy.

Older adults were more loss averse as indicated by significantly larger Delay Discounting Loss Aversion Scores $(M=-.04, S D=.16)$ than younger adults $(M=-.20, S D=$ $.22) ; t(44)=-2.76, p=.01, \eta^{2}=.15, r=.39$ (see Figure 2). However, older adults were less loss averse as indicated by lower Tradeoff Loss Aversion Task Scores $(M=321.33, S D=172.29)$ than younger adults $(M=444.89, S D=50.49) ; F(1,98)=23.68, p<.001, \eta_{p}^{2}=.19$ (see Figure 3). The age difference in Delay Discounting Loss Aversion Scores was attributable to an age difference in participants' discounting of hypothetical monetary losses. Specifically, although younger and older adults did not differ in the degree to which they discounted hypothetical monetary gains $(p=.88)$, older adults' Reversed AUC Losses Scores were significantly smaller $(M=.63, S D=.23)$, showing that older adults discounted hypothetical monetary losses significantly less than younger adults $(M=.79, S D=.17) ; t(44)=2.64, p=.01, \eta^{2}=.14, r=.37$

(see Figure 4). Thus, Hypothesis 3 was supported based on data from the Tradeoff Loss Aversion Task, but was not supported based on data from the Delay Discounting Loss Aversion Task. Specifically, older adults tolerated significantly larger potential monetary losses on the Tradeoff Loss Aversion Task, thus exhibiting less loss aversion than younger adults. However, older adults chose larger delayed hypothetical losses over smaller immediate losses more frequently than younger adults on the Delay Discounting Loss Aversion Task; thus exhibiting greater loss aversion than younger adults.

\section{RQ 4: Calorie advice purchasing and confidence in calorie estimates. Research} question 4 (Are older adults who choose to purchase advice regarding an estimate they are making any more or less confident in their estimates before receiving advice than older adults 
who choose not to purchase advice?) was addressed using logistic regressions. Pre-Advice Confidence Interval Scores from phases 1 and 3 were the predictor variables and decision to purchase advice (yes/no) in phases 2 and 4 were the respective criterion variables. Phase 1 Pre Advice Confidence Interval Score did not predict decision to purchase advice in phase $2 ; b(.00)$ $=.00, p=.16 R^{2}=.00$, odds ratio $=1.00$, Lower $95 \% \mathrm{CI}=.99$, Upper $=1.00$. Furthermore, Phase 3 Pre Advice Confidence Interval Score did not predict decision to purchase advice in phase $4 ; b(.00)=.00, p=.93, R^{2}=.03$, odds ratio $=1.00$, Lower $95 \% \mathrm{CI}=.99$, Upper $=1.00$. Thus, Hypothesis 4 was supported; confidence in calorie estimates prior to purchasing advice was not related to the choice to purchase advice for older adults.

\section{RQ 5: Loss aversion as a mediator of the relation between age and the sunk-cost}

fallacy. Lastly, Research Question 5 (Does loss aversion mediate the relation between age and demonstration of the sunk-cost fallacy on the hypothetical self-report measure?) was addressed using mediation analyses. The traditional approach to testing for mediation recommended by Baron and Kenny (1986) has been noted for having low statistical power (MacKinnon, Lockwood, Hoffman, West \& Sheets, 2002), particularly in smaller samples (Preacher \& Hayes, 2004). Moreover, Preacher and Hayes note that the Baron and Kenny approach involves superfluous criteria, which are not necessary to test for mediation effects, and which increase the chances of a Type II error. Additionally, the Sobel (1982) test has been noted as having better power than the Baron and Kenny approach (Mackinnon et al.). Similarly, the bootstrapping approach (which is nonparametric and thus doesn't rely on normal distribution assumptions), has also been noted as having better power than the Baron and Kenny approach—particularly when variables or the indirect effect in the mediation model have non-normal distributions (Preacher \& Hayes; Shrout \& Bolger, 2002). Thus, the nonparametric bootstrapping method suggested by 
Preacher and Hayes, utilizing 5000 bootstrap resamples, and corresponding Sobel tests were used for the mediation analyses in the present study.

The nonparametric method of mediation analysis described by Preacher \& Hayes (2004) specifies two conditions that must be met in order for loss aversion to act as a mediator of the relation between age group and demonstration of the sunk-cost fallacy. Specifically, (1) there must be a significant relation between age group and demonstration of the sunk-cost fallacy, and (2) the indirect effect of loss aversion must be statistically significant (Preacher \& Hayes).

The Tradeoff Loss Aversion Task Score and the Delay Discounting Loss Aversion Score were tested as potential mediators of the relation between age group and Sunk-Cost Fallacy Vignette Score in separate mediation models. Using age group as the predictor variable and Sunk-Cost Fallacy Vignette Score as the criterion, the first condition was met for mediation (Preacher \& Hayes, 2004) —age group was inversely related to demonstration of the sunk cost fallacy; $\beta=-.28, p=.005$. Additionally, Tradeoff Loss Aversion Task Score was inversely related to age group; $\beta=-.44, p<.001$. Although the Delay Discounting Loss Aversion Score was also related to age group, the direction of the relation was opposite what had been hypothesized — age group was positively related to Delay Discounting Loss Aversion Score; $\beta=$ $.38, p=.01$. Neither the Tradeoff Loss Aversion Task Score, nor the Delay Discounting Loss Aversion Score were related to Sunk Cost Fallacy Vignette Score after controlling for age group in each respective model. Moreover, the relation between age group and Sunk Cost Fallacy Vignette Score was significant when the Tradeoff Loss Aversion Task Score and the Delay Discounting Loss Aversion Score were controlled in each respective model (see Tables 11 and 12). Bootstrapped $95 \%$ confidence intervals included 0 in both models, and Sobel tests were nonsignificant in both models, showing that the relation between age group and demonstration of the 
sunk-cost fallacy was not significantly reduced when either measure of loss aversion was included in either model (Preacher \& Hayes, 2004). Thus, Hypothesis 5 was not supported; neither the Tradeoff Loss Aversion Task Score nor the Delay Discounting Loss Aversion Score mediated the inverse relation between age group and Sunk-Cost Fallacy Vignette Score.

\section{Exploratory Analyses}

Exploratory analyses 1 through 4 were conducted to provide information regarding factors that were related to participants' likelihood of purchasing advice during the Calorie Estimation Task. Exploratory question 1 (Are there age differences in the accuracy of participants' calorie estimates, and does accuracy relate to advice purchasing?) addressed whether people who were able to make more accurate calorie estimates were less likely to purchase advice. An independent samples $t$-test with age group as the independent variable and Pre-Advice Calorie Estimate Accuracy Score as the dependent variable and a logistic regression with Pre-Advice Calorie Estimate Accuracy Score as the predictor and choice to purchase advice (yes/no) as the criterion were conducted. There was no significant difference between younger and older adults' accuracy of calorie estimates in phases 1 and $3(p>.05)$. Additionally, there was no relation between participants' accuracy of calorie estimates and whether or not participants purchased advice $(p>.05)$.

Exploratory question 2 (Are there age differences in participants' degree of confidence in their calorie estimates, and does confidence in calorie estimates relate to advice purchasing?) addressed whether people who were more confident in their calorie estimates were any less likely to purchase advice. An independent samples $t$-test with age group as the independent variable and Mean Pre-Advice Confidence Interval Score as the dependent variable and a logistic 
regression with Mean Pre-Advice Confidence Interval Score as the predictor and choice to purchase advice (yes/no) as the criterion were conducted. Older adults had higher Mean PreAdvice Confidence Interval Scores in phases 1 and $3(M=411.29, S D=39.43)$, suggesting that they were more confident in their estimates than younger adults $(M=356.33, S D=56.48) ; t(98)=$ $-5.64, p<.001, \eta^{2}=.22, r=.49$. A logistic regression indicated that when age group and Mean Pre-Advice Confidence Interval Score were used as predictors of choice to purchase advice (yes/no), there was a significant positive relation between age group and choice to purchase advice $b(.53)=2.17, p<.001, R^{2}=.31$, but there was no relation between Mean Pre-Advice Confidence Interval Score and choice to purchase advice $(p>.05)$.

Exploratory question 3 (Are there age difference in participants' self-reported difficulty in paying their bills, and is there a relation between difficulty paying bills and advice purchasing?) addressed whether people who reported greater difficulty paying bills were less likely to purchase advice. An independent samples $t$-test with Difficulty Paying Bills Score as the dependent variable, and a logistic regression with age group and Difficulty Paying Bills Score as the predictors and choice to purchase advice (yes/no) as the criterion were conducted. Younger adults reported significantly greater difficulty paying their bills $(M=1.30, S D=.95)$ than older adults $(M=.40, S D=.7) ; t(98)=5.23, p<.001, \eta^{2}=.22, r=.47$. Additionally, although there was a significant positive relation between age group and advice purchasing $b(.54)=2.30$, $p<.001, R^{2}=.31$, the relation between Difficulty Paying Bills Score and choice to purchase advice was not significant $(p>.05)$.

Exploratory question 4 (Do older adults who are more loss averse purchase advice less than those who are less loss averse?) addressed whether older adults who were more loss averse were less likely to purchase advice; this analyses was conducted for older adults only due to the 
low rates of advice purchasing among younger adults. Two logistic regressions were conducted, one with Delay Discounting Loss Aversion Score, and the other with Tradeoff Loss Aversion Task Score as the predictor variable. Decision to purchase advice in either phase 2 or phase 4 (yes/no) was the criterion variable in both regressions. Neither the Delay Discounting Loss Aversion Score nor the Tradeoff Loss Aversion Task Score predicted decision to purchase advice for older adults $(p>.05)$.

Exploratory analyses 5 through 8 were conducted to provide information regarding the relations between Calorie Estimate Sunk-Cost Fallacy Scores, Sunk-Cost Fallacy Vignette Scores, loss aversion, and perceptions of the quality of more- vs. less-expensive advice. Additionally, Exploratory Question 6 addressed whether older adults demonstrated the sunk-cost fallacy on the Calorie Estimation Task by weighting more expensive advice more heavily than less expensive advice. Exploratory Question 8 addressed whether participants' perceptions of their ability to estimate calories (i.e., their confidence) were related to their perceptions of advice. Exploratory Question 9 provided information regarding the degree to which participants' final calorie estimates differed from their initial estimates in each condition of the Calorie Estimation Task, and how these differences changed as a function of advice purchasing. Because of the low rates of advice purchasing and subsequent lack of Calorie Estimate Sunk-Cost Fallacy Scores among younger adults, only the older adult sample was included in these analyses.

Exploratory question 5 (For older adults, do the measures of loss aversion relate to the sunk-cost fallacy on the Calorie Estimation Task?) served as a follow-up to the analyses for Research Question 5, which showed that there was no relation between the sunk-cost fallacy on the vignette questionnaire and either measure of loss aversion when age was controlled. Exploratory question 5 addressed whether loss aversion related to demonstration of the sunk-cost 
fallacy when real monetary sunk costs were involved. The Delay Discounting Loss Aversion Score and the Tradeoff Loss Aversion Task Score were both inversely related to Calorie Estimate Sunk-Cost Fallacy Score and Total Advice Adherence Sunk-Cost Fallacy Score, however none of these relations were significant $(p>.05$; see Table 6$)$.

Exploratory question 6 (Do older adults demonstrate the sunk-cost fallacy on the Calorie Estimation Task by weighting advice from the large price of advice condition more heavily than advice from the small price of advice condition? Additionally, for older adults, does the frequency with which the sunk-cost fallacy is demonstrated during the Calorie Estimation Task relate to the how often it is demonstrated on the self-report measure of the sunk-cost fallacy?) addressed whether older adults demonstrated the sunk-cost fallacy on the behavioral measure when real monetary sunk costs were at stake, and addressed whether older adults who demonstrated the sunk-cost fallacy when sunk costs were participant-chosen investments of real money also demonstrated the fallacy when researcher-chosen investments of hypothetical time was at stake. A dependent $t$ test comparing Small and Large Price of Advice Condition WOA scores showed that on average, older adults did not demonstrate the sunk-cost fallacy on the Calorie Estimation Task; there was no significant difference in how closely older adults followed advice from the small and the large price of advice conditions $(p>.05)$. Similarly, a dependent $t$ test comparing Advice Adherence Scores from the small and large price of advice conditions showed that there was no significant difference $(p>.05)$ in older adults' adherence to advice between conditions. Thus, older adults did not demonstrate the sunk-cost fallacy on the Calorie Estimation Task. There was no significant relation between Calorie Estimate Sunk-Cost Fallacy Score Sunk-Cost Fallacy Vignette Score, Sunk-Cost Fallacy Vignette Overinvestment Score, and Normatively Correct Score, $(p>$.05). Similarly, Total Advice Adherence Sunk-Cost Fallacy 
Score was not related to Sunk-Cost Fallacy Vignette Score, Sunk-Cost Fallacy Vignette Overinvestment Score, or Normatively Correct Score $(p>.05$; see Table 6).

Exploratory Question 7 (Do older adults perceive more expensive advice as being of a higher quality than less expensive advice, and for older adults, does demonstration of the sunkcost fallacy on the Calorie Estimation Task relate to perceptions of the quality of advice from the large price of advice condition?) addressed whether people view expensive advice differently than less expensive advice, and whether those perceptions are related to demonstration of the sunk-cost fallacy. There was no significant difference between Quality of Advice Scores from the small price of advice condition and the large price of advice condition $(p>.05)$. Moreover, neither the Calorie Estimate Sunk-Cost Fallacy Score, or the Total Advice Adherence Sunk-Cost Fallacy Score were related to Quality of Advice Score from the large price of advice condition, or from the small price of advice condition $(p>.05)$.

Exploratory Question 8 (is there a relation between participants' confidence in their calorie estimates and their perceptions of advice quality in subsequent phases of the Calorie Estimation Task) addressed the issue of whether participants' perceptions of the quality of advice were related to how much they perceived themselves to know about calories. Correlations showed that for participants who purchased advice, there was no relation between Pre-Advice Confidence Interval Scores and their Quality of Advice Scores from the subsequent phase in either price of advice condition. These correlations were also conducted separately for each age group; all analyses were non-significant $(p>.05)$.

Exploratory Question 9 (Does the degree to which older adults change their final calorie estimates from their initial calorie estimates in a given condition differ based on whether or not 
the older adult purchased advice in the respective condition? That is, do older adults who purchase advice in a given condition change their final calorie estimate in that condition-in comparison to their initial estimate-more than older adults who do not purchase advice?) addressed whether people who purchased advice changed their final estimate (in comparison to their initial estimate) more than people who did not purchase advice. An independent samples $t$ test indicated that among older adults, Small Price of Advice Condition Estimate Difference Scores did not differ significantly as a function of whether the participant purchased advice in that condition $(p>.05)$. However, older adults who did purchase advice in the large price of advice condition changed their final estimates from their initial investments significantly more $(M=844.59, S D=479.71)$ than older adults who did not purchase advice in this condition $(M=$ $505.25, S D=351.09) ; t(48)=2.83, p=.007), \eta^{2}=.14, r=.37$. Additionally, among older adults who purchased advice in both conditions, there was no significant difference between the Small Price of Advice Condition Estimate Difference Scores and the Large Price of Advice Condition Estimate Difference Scores $(p>.05)$.

Exploratory analyses 10 through 12 were conducted to provide preliminary information about the convergent validity of both measures of loss aversion, as well as the relation between loss aversion and future time perspective, which may play a role in older adults' increased attention to positive information (Carstensen et al., 1999; Mather \& Carstensen, 2005). Moreover, Exploratory Question 12 addressed whether the delay discounting of gains and losses task, which required participants to consider the future, related to future time perspective, which has been shown to become more limited with age (Lang \& Carstensen, 2002).

Exploratory Question 10 (Do Delay Discounting Loss Aversion Scores relate to Tradeoff Loss Aversion Task Scores?) addressed whether these two measures assessed the same construct. 
A correlation between the two variables showed no significant relation $(p>.05$; see Table 5). This correlation was also conducted independently for both younger and older adults; the two measures of loss aversion were unrelated when either only younger or older adult data was assessed ( $p>.05$; see Table 6). Additionally, a Fisher's r-to-z transformation (Fisher, 1921) showed that the correlations between Delay Discounting Loss Aversion Scores and Tradeoff Loss Aversion Scores did not differ significantly between younger and older adults; $z^{\prime}=.00, p>$ .05 .

\section{Exploratory Question 11 (Do either of the measures of loss aversion relate to The} Spendthrift-Tightwad Scale?; Rick et al., 2008) addressed whether loss aversion was related to aversion to spending money; two constructs that should be related, conceptually speaking. Neither the Delay Discounting Loss Aversion Score, nor the Tradeoff Loss Aversion Task Score was related to Spendthrift-Tightwad Scale Scores $(p>.05)$. These correlations were also conducted independently for each age group; none of these correlations were significant $(p>.05$; see Table 6). However, the relation between Tradeoff Loss Aversion Task Score and SpendthriftTightwad Scale Scores did approach significance for younger adults $(r=-.24, p=.08$; see Table 6). A Fisher's r-to-z transformation (1921) showed that the correlations between Tradeoff Loss Aversion Task Score and Spendthrift-Tightwad Scale Scores did not differ significantly between younger and older adults; $z^{\prime}=-.60, p>.05$.

Also, there was no significant relation between The Delay Discounting Loss Aversion and Spendthrift-Tightwad Scale Scores for either younger or older adults $(p>.05$; see Table 6). A Fisher's r-to-z transformation (1921) showed that the correlations between The Delay Discounting Loss Aversion and Spendthrift-Tightwad Scale Scores did not differ significantly between younger and older adults; $z^{\prime}=.20, p>.05$. 
Exploratory Question 12 (Are there age differences in future time perspective, and does future time perspective relate to Tradeoff Task Loss Aversion Score, Delay Discounting Loss Aversion Score, or Reversed Area Under the Curve Gains or Losses Scores?) addressed whether future time perspective (FTP); a construct based on peoples' perceptions of the future, differed by age group (e.g., Lang \& Carstensen, 2002). Exploratory Question 12 also addressed whether FTP (a variable that is related to age, and which may relate to the positivity effect that older adults experience; Carstensen et al., 1999; Lang \& Carstensen; Mather \& Carstensen, 2005) was related to loss aversion, which was inversely related to age in the present study. Additionally, Exploratory Question 12 addressed whether delay discounting of gains and losses—which involves peoples' perceptions of the future - related to FTP. More specifically, people with more limited FTP may be more likely to choose smaller immediate gains and larger delayed losses if they perceive that they will not be alive to experience either the delayed gains or losses. Alternatively, if individuals with more limited FTP are also less averse to losses, they may be more likely to choose larger delayed gains and smaller immediate losses. Younger adults had significantly more expansive FTP $(M=5.61, S D=.86)$ than older adults $(M=3.71, S D=.95)$; $t(98)=10.52, p<.001, \eta^{2}=.53, r=.73$.

The Tradeoff Task Loss Aversion Score positively related to Future Time Perspective (FTP) Score $(r=.38, p<.001$; see Table 5). However, the Delay Discounting Loss Aversion Score was inversely related to FTP Score $(r=-.50, p<.001$; see Table 5$)$. The Reversed Area Under the Curve (AUC) Gains Score was not related to FTP Score $(p>.05)$. However, the Reversed AUC Losses Score was positively related to FTP Score $(r=.37, p=.01)$, suggesting that people with more expansive FTP discounted delayed losses more (i.e., chose smaller immediate losses over larger delayed losses more frequently). These correlations were also 
conducted separately for each age group; Delay Discounting Loss Aversion Score was inversely related to FTP Score for younger $(r=-.50, p=.01)$, but not for older adults $(p>.05)$. Tradeoff Task Loss Aversion Scores were not related to FTP Scores for either younger or old adults. ( $p>$ .05 ; see Table 6).

Fisher's r-to-z transformations (1921) were conducted for each pair of correlations to assess whether correlations differed between age groups. Fisher's r-to-z transformations indicated that the correlations between FTP and Tradeoff Task Loss Aversion Scores, Delay Discounting Loss Aversion Scores, Reversed AUC Gains Score, and Reversed AUC Losses Score did not differ significantly between younger and older adults $(p>.05)$.

Exploratory questions 13 and 14 addressed the relations between participants' knowledge of nutritional information and numerical ability, and their performance on several tasks in this study. Specifically, Exploratory Question 13 addressed whether the exploratory measure of health habits exhibited any convergent validity with an existing measure (i.e., Steptoe et al., 1995). Exploratory Question 13 also provided information about the relation between participants' health habits and concern with nutrition and their ability to make accurate calorie estimates on the Calorie Estimation Task, as well as their confidence in the accuracy of their estimates. Exploratory Question 14 provided information about the relation between participants' perceived numerical abilities and their ability to make accurate calorie estimates on the Calorie Estimation Task, as well as their confidence in the accuracy of their estimates. Exploratory Question 14 also provided information regarding the relation between participants' perceived numerical abilities and their performance on the delay discounting and tradeoff tasks and the self-report measure of the sunk-cost fallacy; all of which required participants to review numerical content. 
Exploratory Question 13 (Does the exploratory measure of health habits relate to the weight control subscale of the Food Choice Questionnaire?; Steptoe et al., 1995, and do participants' health habits relate to their ability to make accurate calorie estimates or their confidence in the accuracy of their estimates?) was addressed with correlations. The exploratory Health Habits Questionnaire was positively related to the Food Choice Questionnaire; $r=.63, p$ $<.001$ (see Table 7), suggesting that the Health Habits Questionnaire has convergent validity (Campbell \& Fiske, 1959). The Food Choice Questionnaire was not related to Pre-Advice Calorie Estimate Accuracy Scores or Mean Pre-Advice Confidence Interval Score $(p>.05)$. However, the Health Habits Questionnaire was positively related to Pre-Advice Calorie Estimate Accuracy Scores $(r=.25, p=.01)$, and was positively related to Mean Pre-Advice Confidence Interval Score; $r=.22, p=.02$ (see Table 7). Thus, better health habits as assessed by the Health Habits Questionnaire were positively related to the ability to accurately estimate caloric values of food items, and were also positively related to participants' confidence in their estimates, further suggesting that the Health Habits Questionnaire has convergent validity (Campbell \& Fiske).

These correlations were also conducted for each age group separately (see Table 8). The Food Choice Questionnaire was not related to Mean Pre-Advice Confidence Interval Score for either age group $(p>.05)$, and Fisher's r-to-z transformation (1921) showed that the correlations did not differ significantly between age groups $(p>.05)$. However, the Food Choice Questionnaire was positively related to Pre-Advice Calorie Estimate Accuracy Scores for younger adults $(r=.35, p=.01)$, but not for older adults $(p>.05)$, and a Fisher's r-to-z transformation showed that the correlations differed significantly between younger and older adults; $z^{\prime}=2.01 ; p=.02$. The Health Habits Questionnaire was not related to Mean Pre-Advice Confidence Interval Score for either age group $(p>.05)$, and a Fisher's r-to-z transformation 
showed that the correlations did not differ significantly between age groups. The Health Habits Questionnaire was related to Pre-Advice Calorie Estimate Accuracy Scores $(r=.29, p=.03)$ for younger adults, but not for older adults $(p>.05)$, these correlations did not differ significantly between age groups $(p>.05)$. The Health Habits Questionnaire was positively related to the Food Choice Questionnaire for both younger adults $(r=.66, p<.001)$, and for older adults $(r=$ $.57, p<.001$; see Table 8$)$; these correlations did not differ significantly between age groups $(p>$ $.05)$.

Exploratory Question 14 (Does numerical ability relate to participants' ability to make accurate calorie estimates, their confidence in their estimates, or their performance on the measures of loss aversion or the sunk-cost fallacy?) was addressed with correlations. Subjective Numeracy Score (SNS; Fagerlin, Zikmund-Fisher, Ubel, Jankovic, Derry, \& Smith, 2007) was not significantly related to Pre-Advice Calorie Estimate Accuracy Score, Mean Pre-Advice Confidence Interval Score, Delay Discounting Loss Aversion Score, Tradeoff Loss Aversion Task Score, Sunk-Cost Fallacy Vignette Score, Calorie Estimate Sunk-Cost Fallacy Score, or Total Advice Adherence Sunk-Cost Fallacy Score ( $p>.05$; see Table 5). These correlations were also conducted separately for each age group; all correlations remained non significant $(p>.05$; see Table 6), and Fisher's r-to-z transformations (1921) indicated that none of the correlations were significantly different between age groups $(p>.05)$.

There was significant inverse relation between SNS and Reversed AUC Gains Scores ( $r$ $=-.33, p=.03$; see Table 5), suggesting that people with higher subjective numerical ability discounted delayed gains less than people with lower subjective numerical ability. There was also an inverse relation between SNS and Reversed AUC Losses Score that approached, but not reach statistical significance $(r=-.28, p=.06$; see Table 9). Additionally, the Reversed AUC 
Gains Scores were positively related to Reversed AUC Losses Scores $(r=.47, p=.001)$, suggesting a positive relation between delay discounting of monetary gains, and of losses (see Table 9). These correlations were also conducted separately for each age group; correlations between SNS Score and Reversed AUC Gains Score and Reversed AUC Losses Score were both non significant for younger adults (see Table 10). For older adults, the correlation between SNS Score and Reversed AUC Gains Score was significant ( $r=-.43, p=.04$ ), as was the correlation between Reversed AUC Gains Score and Reversed AUC Losses Score $(r=.64, p=.001)$. However, the correlation between SNS Score and Reversed AUC Losses Score was nonsignificant for older adults $(p>.05$; see Table 10). Fisher's r-to-z transformations (1921) indicated that the correlations between SNS and Reversed AUC Gains Score and between SNS and Reversed AUC Losses Score did not differ significantly between younger and older adults $(p$ $>$.05). Similarly, the correlations between Reversed AUC Gains Score and Reversed AUC Losses Score did not differ significantly between age groups $(p>.05)$.

Exploratory Questions 15 through 17 were conducted to assess the utility of the advice that participants purchased in terms of whether it was related to an improved ability to win money on the Calorie Estimation Task, age differences in winnings from the Calorie Estimation Task, and relations between winnings on the Calorie Estimation Task and demonstration of the sunk-cost fallacy on the Calorie Estimation Task.

Exploratory Question 15 (Did participant's final winnings from the Calorie Estimation Task differ based on whether participants purchased advice in either or both price of advice conditions?) addressed whether the advice was useful in helping participants to win more money during the Calorie Estimation Task. Exploratory Question 15 was addressed with independent samples $t$ tests. Final Winnings Scores were not significantly different for participants who 
purchased advice in the small price of advice condition compared to those who did not $(p>.05)$. Similarly, Final Winnings Scores did not differ significantly between participants who did and who did not purchase advice in the large price of advice condition $(p>.05)$. Additionally, Final Winnings Scores did not differ significantly between participants who purchased advice in both conditions, and those who did not purchase advice $(p>.05)$. These analyses were also conducted independently for each age group, all results were non-significant.

Exploratory Question 16 (Were there age differences between younger and older adults in how much money was earned through accurate estimates on the Calorie Estimation Task?) was addressed using an independent-samples $t$ test. There was no significant difference between younger and older adults' Final Winnings Scores $(p>.05)$.

Exploratory Question 17 (Is there a relation between advice following and winnings from accurate estimates on the Calorie Estimate Task, and is there a relation between demonstration of the Sunk-Cost Fallacy on the Calorie Estimation Task and winnings?) was addressed with correlations. There was no significant relation between either WOA Scores or Advice Adherence Scores from either the small or large price of advice conditions and Final Winnings Scores $(p>$ .05). Moreover, there was no significant relation between Final Winnings Scores and either the Calorie Estimate Sunk-Cost Fallacy Scores, or the Total Advice Adherence Sunk-Cost Fallacy Scores $(p>.05)$.These analyses were also conducted independently for each age group; all results remained non-significant.

\section{Discussion}

The primary objectives of the present study were to assess age differences in how frequently people demonstrated the sunk-cost fallacy, overinvested after demonstrating the 
fallacy, and made normatively correct decisions, as well as age differences in loss aversion, and whether loss aversion mediated the relation between age and demonstration of the sunk-cost fallacy. Furthermore, this study assessed whether an established self-report measure of the sunkcost fallacy (i.e., Strough et al., 2008; 2011b) related to peoples' behavior when real, rather than hypothetical sunk costs were at stake. This study provides a systematic replication (Sidman, 1960) of work by Strough and colleagues (2008; 2011b) and complements prior research (i.e., Bruine de Bruin et al., 2007). Additionally, the present study extends prior findings by showing age differences in loss aversion, and discounting of delayed losses, and by assessing whether loss aversion mediates the relation between age and demonstration of the sunk-cost fallacy. Also, the present study assessed the relations between age and a variety of measures related to decisionmaking.

\section{Overview of Discussion}

Specifics of the methods, results, and relevance of the present study are discussed below, including: (1) a discussion of factors related to participants' willingness to pay for advice (i.e., their willingness to make initial investments), perceived quality of advice, the relation between hypothetical and real willingness to pay, and the relation between advice purchasing and calorie estimates (2) a discussion of age differences in demonstration of the sunk-cost fallacy, degree of overinvestment, normatively correct decisions, the two measures of the sunk-cost fallacy used in the present study and the relation between the two, (3) a discussion of loss aversion as a mediator of the relation between age and the sunk-cost fallacy, and a discussion of the two measures of loss aversion used in the present study, (4) a discussion of age differences in the discounting of delayed gains and losses, age differences in loss aversion, and the relation of each to future time 
perspective, (5) a general discussion of the implications and limitations of the present study, and possible directions for future research.

\section{Rates of Advice Purchasing and Willingness to Pay}

Prior studies have shown that younger adults report that they are more willing to pay for a variety of goods ranging from informative leaflets (e.g., Botelho \& Pinto, 2001), to sunglasses (e.g., Blumenschein et al., 1998) when the payment is hypothetical, rather than real money. Similarly, other studies have suggested that middle-aged adults' willingness to pay is higher when the payment is hypothetical, rather than real (Blumenschein et al., 1998). Because willingness to make an initial investment is a pre-requisite for demonstration of the sunk-cost fallacy, willingness to pay money (or invest other resources) is a critical issue when studying the sunk-cost fallacy.

When given the opportunity to purchase advice that could potentially be used to earn more money in the present study, older adults purchased advice more frequently than younger adults in both the small, and the large price of advice conditions. The frequency with which younger adults purchased advice did not correspond with the findings of Gino (2008), who reported that approximately half of her younger adult participants purchased advice in her procedure. Several of the analyses in the present study addressed possible explanations for the age difference in rates of advice purchasing (e.g., Exploratory Questions 1, 2, 3, 4).

When people choose whether to pay for a resource, characteristics of both the person and the resource can relate to the person's willingness to pay for the resource. For instance, people who have a greater need for a particular resource are generally more willing to pay for that resource (e.g., Tambour \& Zethareus, 1998). Thus, younger adults' unwillingness to pay for 
advice relative to older adults in the present study might be indicative of a low need for advice on caloric content of food items among younger adults, a high need among older adults, or both. Results from analyses that addressed these issues are discussed next.

The present study included two measures that served as proxies of participants' need for advice in the Calorie Estimation Task - namely, participants' confidence in their calorie estimates, and the accuracy of their calorie estimates. Participants who had very little confidence in their estimates, or who could not accurately estimate caloric values could be said to have a greater need for advice when making calorie estimates. However, in the present study, results suggested that the age differences in advice purchasing were not related to differences in participants' need for advice; neither younger or older adults' confidence in the accuracy of their calorie estimates prior to purchasing advice was related to their likelihood of purchasing advice (Exploratory Question 2).

Additionally, although there was a significant age difference in participants' estimate confidence (Exploratory Question 2), it was older adults who were more confident in their calorie estimates. This is interesting because it shows that even though older adults expressed more confidence in their ability to make accurate calorie estimates, they were nonetheless more willing to pay for advice regarding those calorie estimates than younger adults. Moreover, there was no relation between the accuracy of younger or older adults' calorie estimates and their willingness to purchase advice, and there was not a significant difference between younger and older adults in terms of the accuracy of their calorie estimates (Exploratory Question 1).

Another potential motivating factor in peoples' willingness to pay for advice may have been peoples' expectations regarding whether the advice would help them make more accurate 
calorie estimates, thereby winning them more money from the Calorie Estimation Task. There was no measure in the present study to assess whether participants thought that advice would help them win more money. However, analyses from Exploratory Question 15 showed that final winnings from the Calorie Estimation Task did not differ based on whether participants purchased advice in either or both of the conditions in which it was available for either the whole sample, or either age group. That is, both younger and older adults who did not purchase advice in the small or large price of advice conditions, or both, received winnings from the Calorie Estimation Task that were not significantly different than the winnings received by younger or older adults who did purchase advice. Furthermore, analyses from Exploratory Question 16 showed that there was no significant difference between younger and older adults in winnings from the Calorie Estimation Task. Additionally, analyses from Exploratory Question 17 showed that there was no significant relation between winnings from the Calorie Estimation Task and demonstration of the sunk cost fallacy on the Calorie Estimation Task for either age group, or for the whole sample. Similarly, analyses from Exploratory Question 17 showed that there was no significant relation between either WOA Scores or Advice Adherence Scores from either the small or large price of advice condition and subsequent winnings on the Calorie Estimation Task for either younger or older adults, or the whole sample.

The fact that advice purchasers earned as much as non-purchasers (Exploratory Question 15), combined with the fact that advice following was not related to final winnings (Exploratory Question 17), suggests that the non-significant difference in final winnings between advice purchasers and non-purchasers was not attributable to less knowledge of calories and subsequent utilization of advice on the part of advice purchasers. This finding is further supported by results showing that participants' accuracy and confidence in their calorie estimates was not related to 
advice purchasing (Exploratory Questions 1 and 2, respectively). The lack of utility of advice purchasing and following would provide a potentially valid explanation of why relatively few participants purchased advice were it not for the fact that participants had no means of anticipating this lack of utility. Moreover, because participants were informed that advice originated from an expert, they were given the impression that purchasing and following advice would be useful in terms of winning more money. Additionally, the non-significant relation between demonstration of the sunk-cost fallacy and winnings (Exploratory Question 17) shows that in terms of winnings, there was little or no utility in either demonstrating or not demonstrating the sunk-cost fallacy by following more expensive advice more closely than less expensive advice. However, participants had no means of assessing whether there was any utility in either demonstrating or not demonstrating the sunk-cost fallacy by following more expensive advice more closely than less expensive advice. Thus, from the perspective of participants in the present study, the only information that might have been perceived as indicating a differing level of utility in purchasing and/or following advice was the price of the advice. Additionally, based on the data from the present study, it is impossible to assess whether the participants who did purchase advice would have won less money had they not purchased advice.

Characteristics of the good or resource that a person pays for can be related to their willingness to pay (Bushong, King, Camerer \& Rangel, 2010); prior research has shown that the availability of information about the quality of a good is related to peoples' willingness to pay. For instance, people in a study by Blomquist and Whitehead (1998) were willing to pay more money for wetland preservation when the description of the wetland was of a higher quality. The advice that people could purchase in the present study was presented as a resource that would 
presumably be of a relatively high quality (i.e., the advice was presented as originating from an expert).

Moreover, peoples' perceptions of a good or resource may change as a result of making an initial investment, or as a result of the price of the of the good. For instance, Arkes and Hutzel (2000) showed that younger adults' estimates of the probability of success resulting from continued investment in a product were inflated when a prior investment had been made in the product (i.e., a sunk cost). Similarly, the price of a good is often positively related to peoples' perceptions of the quality of the good (i.e., people tend to perceive more expensive items as being of a higher quality than less expensive items; Rao \& Monroe, 1989; Teas \& Agarwal, 2000).

Although there is evidence to suggest that people perceive higher-priced items to be of higher quality, and courses of action to have a higher probability of success following a sunk cost, most of this research is based on younger adult samples (e.g., Arkes \& Hutzel, Teas \& Agarwal). If younger adults in the present study assumed that the advice would be of low quality based on its price, this may partly explain why younger adults generally did not purchase advice. Unfortunately, because perceptions of advice quality were assessed only after a participant purchased advice in the present study, it was not possible to assess whether younger adults perceived the quality of advice any differently than older adults, or whether advice quality perceptions predicted decisions to purchase advice. However, the mean Quality of Advice Scores for the small and large price of advice conditions were 4.51 and 4.45 , respectively for the entire sample (out of a possible range of 1-6, with higher scores indicating higher perceived quality; see Table 3). A mean Quality of Advice Score of 3 would indicate that the participant had a neutral perception of the advice. Thus, on average, participants who purchased advice in the present 
study viewed the advice as being relatively accurate and originating from a person who knew more about calories than they did (see Appendix A). Although participants had a generally positive perception of the advice they purchased, older adults' perceptions of the quality of advice did not differ as a function of the price of the advice (see Exploratory Question 7).

Additionally, perceptions of a good might depend on the potential consumer's perceived need for the good. For instance, participants in the present study who perceived themselves as not being knowledgeable about calories, as indicated by lower confidence in the accuracy of their calorie estimates (and thus a greater need for advice) might have had a better perception of the quality of advice. Exploratory Question 8 showed that there was no relation between participants' Pre-Advice Confidence Interval Scores and their Quality of Advice Scores from the subsequent phase in either price of advice condition. Thus, in the present study, participants' perceived confidence in their ability to accurately estimate calories did not relate to their perceptions of the advice pertaining to calories.

Other research has shown that people are more willing to pay when the item that they are paying for is physically present (as opposed to being represented in a photo or with text; Bushong et al., 2010). The good that people could purchase in the present study (i.e., advice) is a commodity that can only be presented in a limited number of ways (i.e., text, spoken words). If younger adults view non-tangible commodities such as advice less favorably than older adults, they may be less willing to pay for it. Indeed, it might be possible that younger adults have less experience purchasing intangible products, such as advice. Older adults, on the other hand, may have more experience purchasing such goods - advice from financial advisors, attorneys and medical doctors, as well as the purchasing of stocks and bonds are all examples of investments in intangible goods that older adults may have more experience with than younger adults. If older 
adults are more familiar with investing in intangible goods than younger adults, they may be more likely to make such investments - as they did in the present study by purchasing advice. Unfortunately, experience with investing in intangible goods was not measured in the present study, and thus should be explored in future research.

The procedure in the Calorie Estimation Task involved having participants make calorie estimates twice within each price of advice condition and assessing whether people demonstrated the sunk-cost fallacy on the Calorie Estimation Task by changing their final estimate to correspond more closely with advice when the advice was more expensive compared to less expensive advice. However, the effects of purchasing advice in each condition on the degree to which participants changed their final estimate compared to their initial estimate were also assessed (see Exploratory Question 9). In the small price of advice condition, the difference between older adults' initial and final calorie estimates did not differ based on whether advice was purchased. Moreover, there was no significant difference between older adults' Small Price of Advice Condition Estimate Difference Scores and their Large Price of Advice Condition Estimate Difference Scores. However, older adults who purchased advice in the large price of advice condition did change their final estimates from their initial estimates significantly more than older adults who did not purchase advice in this condition (see Exploratory Question 9). Thus, older adults who purchased advice also changed their final estimates from their initial estimates to a greater extent than older adults who did not purchase advice, but only in the large price of advice condition. One possible interpretation of this is that older adults followed more expensive advice more closely than less expensive advice (thus committing the sunk-cost fallacy). However, as noted in Exploratory Question 6, this was not the case-older adults did not follow more expensive advice more closely than less expensive advice (based on WOA 
Scores), nor did they adhere to more expensive advice more closely than less expensive advice (based on Advice Adherence Scores). Thus, the results of Exploratory Question 9 suggest that although older adults changed their final estimates from their initial estimates more when they purchased more expensive advice (compared to less expensive advice), the change in estimates was not necessarily indicative of greater advice following.

\section{The Sunk-Cost Fallacy, Normatively Correct Decisions, and Overinvestment}

The findings of the present study underscore the reliability of adult age differences in the sunk-cost fallacy and normatively correct decisions. Compared to younger adults, older adults consistently demonstrate the sunk-cost fallacy less frequently, overinvest less when they do demonstrate the sunk-cost fallacy, and make normatively correct decisions more frequently, when responding to a self-report measure that is presented in person (Strough et al., 2008), over the phone (Strough et al., 2011b), and in the case of the present study, on a computer. The results of the study complement the findings of Bruine de Bruin and colleagues (2007), who showed that older adults are more likely to ignore prior investments when deciding whether to continue a course of action than younger adults.

With the notable exceptions of a field study conducted by Arkes and Blumer (1985), a laboratory experiment conducted by Gino (2008), and an analysis of archival data by Staw and Hoang (1995), little research related to the sunk-cost fallacy has assessed peoples' behavior when real monetary sunk costs are at stake. Moreover, this is perhaps the first study to directly compare an established self-report measure of the sunk-cost fallacy to a behavioral measure. Although an insufficient number of younger adults purchased advice in the Calorie Estimation 
Task to assess the effects of real monetary sunk costs in the present study, older adults did purchase advice frequently enough to assess the effects of real monetary sunk costs.

For older adults, there was a positive, but non-significant relation between the self-report measure (i.e., the Sunk Cost Vignette Questionnaire), and the behavioral measure (i.e., the Calorie Estimation Task) of the sunk-cost fallacy. Because this analysis was based on a limited number of participants due to low rates of advice purchasing (see Table 6), low power was likely an issue.

Moreover, the positive but non-significant relation between the two measures of the sunk-cost fallacy among older adults should be considered in the context of several important differences between the two measures. First, the Sunk Cost Vignette Questionnaire involved hypothetical sunk costs of both time and money, which were not chosen by the participants. However, the Calorie Estimation Task allowed participants to decide to commit investments (i.e., sunk costs) of real money. Secondly, the outcomes of either committing more or less resources in response to sunk costs were ambiguous to participants during both the Sunk Cost Vignette Questionnaire, and the Calorie Estimation Task. However, any perceptions that participants may have had of the potential outcomes were entirely hypothetical in relation to the Sunk Cost Vignette Questionnaire, whereas participants were aware that outcomes of the Calorie Estimation Task were real (i.e., they were aware that they could lose or earn real money). Third, demonstration of the sunk-cost fallacy differed between the measures. Specifically, for the Sunk Cost Vignette Questionnaire, the sunk-cost fallacy was demonstrated when a participant indicated a greater continued hypothetical investment of time in hypothetical scenarios where the sunk cost was either present or larger compared to hypothetical scenarios where it was either absent or smaller. The sunk-cost fallacy was demonstrated during the Calorie Estimation Task 
when a participant followed more expensive advice more closely than less expensive, but equally accurate advice.

Although the two measures of the sunk-cost fallacy used in the present study differed in a number of ways, prior research has suggested that the sunk cost fallacy may be demonstrated across a variety of measures involving investments of time, money, effort, and adherence to advice (e.g., Cunha \& Caldieraro, 2009; Friedman, Pommerenke, Lukose, Milam, \& Huberman, 2007; Gino, 2008; Navarro \& Fantino, 2009; Strough et al., 2011b; Ting, 2011). Thus, the positive relation between the behavioral measure involving real sunk costs and the self-report measure involving hypothetical sunk costs may be dependent in part on the number and types of scenarios included in the self-report measure.

\section{Loss Aversion as a Mediator of Age Differences in the Sunk-Cost Fallacy}

Mediation models. Older adults were less loss averse on the Tradeoff Loss Aversion Task and more loss averse on Delay Discounting Loss Aversion Task than younger adults. Two mediation models were conducted to test whether either measure of loss aversion mediated the relation between age group and demonstration of the sunk-cost fallacy on the Sunk Cost Vignette Questionnaire. The mediation models in the present study used the nonparametric bootstrapping approach (Preacher \& Hayes, 2004) and Sobel (1982) tests. Each analysis indicated that neither measure of loss aversion mediated the relation between age group and demonstration of the sunk-cost fallacy (see Tables 11 and 12).

However, it should be noted that insufficient power may have played a role in the nonsignificant mediation when Delay Discounting Loss Aversion Score was used as a potential mediator. Specifically, because of the removal of nonsystematic delay discounting data, the 
number of cases included in these analyses was especially low (i.e., 22 younger adults and 23 older adults; see Table 11). Furthermore, this is the first study to test whether loss aversion mediates the relation between age and demonstration of the sunk-cost fallacy - thus, further exploration of the relations between age, loss aversion, and the sunk-cost fallacy is warranted.

Loss aversion measures. There was not a significant relation between scores from the Delay Discounting and the Tradeoff Loss Aversion Tasks in the present study. However, this finding should be interpreted with caution because the correlation was based on a limited number of participants due to nonsystematic delay discounting data (see Tables 5 and 6). Additionally, older adults had significantly higher Delay Discounting Loss Aversion Scores, and significantly lower Tradeoff Task Loss Aversion Scores than younger adults, suggesting that each measure was assessing a different construct.

Additionally, for older adults, there was no relation between either measure of loss aversion and demonstration of the sunk-cost fallacy on the Calorie Estimation Task. However, this finding should also be interpreted with caution, as both correlations were based on a limited number of cases due to nonsystematic delay discounting and low rates of advice purchasing during the Calorie Estimation Task. Specifically, the correlations between Tradeoff Loss Aversion Task Scores and both the Calorie Estimate Sunk-Cost Fallacy Score and the Total Advice Adherence Sunk-Cost Fallacy Score were based on 26 participants, whereas the correlations between Delay Discounting Loss Aversion Score and both the Calorie Estimate Sunk-Cost Fallacy Score and the Total Advice Adherence Sunk-Cost Fallacy Scores were based on 8 participants - thus, low power was likely a factor (see Table 5). Subsequent power analyses utilizing G*Power software confirmed that 8 or 26 participants would produce insufficient power for these analyses (Faul, Erdfelder, Buchner \& Lang, 2009). 
Neither measure of loss aversion was significantly related to the Spendthrift-Tightwad Scale (STS; Rick et al., 2008). However, the correlations between the Delay Discounting Loss Aversion Scores and STS Scores were all positive (for the whole sample, and each age group), whereas the correlations between the Tradeoff Loss Aversion Task Score and the STS Scores were all inverse (see Tables 5 \& 6). The inverse relation between Tradeoff Loss Aversion Task Scores and STS Scores approached, but did not reach significance for younger adults $(p=.08$; see Table 6). These results suggest that although the correlations were all non-significant, both younger and older adults who had higher scores on the Delay Discounting Loss Aversion Task reported less difficulty spending money on the STS. On the other hand, both younger and older adults who demonstrated greater loss aversion on the Tradeoff Loss Aversion Task reported greater difficulty spending money on the STS. The STS is designed to measure how easy or difficult people find it to spend money (i.e., how averse people are to spending). Specifically, the STS includes items where respondents rate how easy and difficult they find it to spend money, and also how similar they view themselves to hypothetical characters who either spend money easily or have difficulty spending money. The Tradeoff Loss Aversion Task, on the other hand, was intended to measure aversion to losing money. Although spent money is not necessarily the same as lost money, spent money is similar to lost money in the sense that it's no longer usable to the person who originally had it. Conceptually, a person who indicates that they are only willing to tolerate relatively small monetary losses (and who are thus relatively averse to losing money) on the Tradeoff Task, would presumably also report that they are relatively averse to spending money on the STS. Thus, the two measures are designed to assess separate, but conceptually related constructs. Taken together, these findings suggest that there is preliminary evidence that the Tradeoff Loss Aversion Task used in the present study exhibited convergent 
validity (Campbell \& Fiske, 1959) with the STS, but that the Delay Discounting Loss Aversion Task did not.

Kahneman and Tversky (1979) defined loss aversion as an aversion to "50-50" bets involving equally probable gains and losses of equivalent values. According to Kahneman and Tversky, loss aversion progressively increases as the absolute magnitude of the stakes in the bet increase. Conceptually speaking, the Tradeoff Loss Aversion Task Scores correspond with Kahneman and Tversky's definition more closely than the Delay Discounting Loss Aversion Task. Specifically, the Tradeoff Loss Aversion Task Scores were derived by assessing the magnitude of potential monetary loss that a person was willing to tolerate on a 50-50 bet that had an equal chance of resulting in a large monetary gain, which remained constant, or a smaller monetary loss that increased across trials, incrementally approaching the magnitude of the potential gain. People who opted out of the bet at smaller potential loss values and chose a different 50-50 bet with smaller potential gains and losses were more loss averse than people who tolerated larger potential losses (see appendix D). The Delay Discounting Loss Aversion Scores, on the other hand, were derived by taking the difference between peoples' discounting of delayed gains and losses — a greater preference for small immediate monetary rewards and larger delayed monetary losses was indicative of loss aversion in the present study (see Appendix C).

Additionally, the Delay Discounting Loss Aversion Task used in the present study was relatively cumbersome (i.e., it involved repetitive choices across 8 delays, each of which were presented with ascending and descending monetary values). Moreover, prior studies have not used delay discounting procedures to assess loss aversion, and the temporal component of the procedure complicates its interpretation as a measure of loss aversion. The Tradeoff Loss Aversion Task, on the other hand, did not involve a temporal component, and was completed in a 
shorter number of trials than the Delay Discounting Loss Aversion Task. Thus, the Tradeoff Loss Aversion Task may have resulted in less participant fatigue than the Delay Discounting Loss Aversion Task.

Although both the Delay Discounting and the Tradeoff Loss Aversion Tasks were both adapted from prior research (Green et al., 1994; Fennema \& Van Assen, 1999, respectively), each were novel measures of monetary loss aversion. Specifically, although many studies have assessed discounting of delayed gains and losses, this is perhaps the first study to use the difference between discounting of delayed losses and gains as a measure of monetary loss aversion. However, the positive (although non-significant) relation between the Delay Discounting Loss Aversion Scores and difficulty controlling spending as assessed on the Spendthrift Tightwad Scale (STS; Rick et al., 2008) suggest that this is not a valid assessment of loss aversion. Additionally, the Tradeoff Task used in this study was adapted from Fennema and Van Assen, who had originally used the task to assess utility functions. In the present study, the Tradeoff Task was simplified by removing information pertaining to probabilities of gains and losses (which Fennema and Van Assen had used), which were irrelevant to the purposes of measuring loss aversion in the present study. The Tradeoff Task Loss Aversion Scores were inversely (although not significantly) related to difficulty controlling spending as assessed on the STS — thus, although there is preliminary evidence to suggest that the Tradeoff Loss Aversion Task is a valid assessment of monetary loss aversion, further research should be conducted to clarify the predictive and convergent validity of this measure.

\section{Delay Discounting, Loss Aversion and Future Time Perspective.}


The age difference in younger and older adults' Delay Discounting Loss Aversion Scores was attributable to an age difference in discounting of delayed hypothetical monetary losses (but not gains). Specifically, although older adults discounted both delayed gains and losses less than younger adults, the age effect was significant only for delayed losses, and not delayed gains. That is, younger adults discounted delayed losses significantly more than older adults (i.e., younger adults chose smaller immediate losses over larger delayed losses more frequently than older adults).

With the exception of Löckenhoff and colleagues' 2011 study, this is one of the few studies to assess adult age differences in delay discounting of monetary losses. Similar to the present study, Löckenhoff and her colleagues found that age was inversely associated with discounting of delayed monetary gains and losses. However, unlike the present study, Löckenhoff and her colleagues found that the age difference in discounting was only significant for gains (whereas the age difference in discounting was only significant for delay discounting of losses in the present study). However, the findings of Löckenhoff and her colleagues are not directly comparable with the findings of the present study because of a number of differences in the procedures of the respective studies. First, the delay discounting task used by Löckenhoff and her colleagues involved choices regarding gains and losses of real money, whereas the monetary amounts involved in the delay discounting task used in the present study were all hypothetical. Second, Löckenhoff and her colleagues used a procedure in which the immediate amount (for both gains and losses) remained constant, while the delayed amount varied, as did the length of the delay. In the present study, the immediate amount varied (for both gains and losses), while the delayed amount remained constant, and the length of delays increased across trials. Third, the range of monetary and delay values were more restricted in Löckenhoff and colleagues' study 
compared to the present study; the delayed monetary values ranged from $\$ 4.74$ to $\$ 7.50$, while the delay values ranged from 7 to 180 days, with a total of 4 different delays. By contrast, the immediate monetary values in the present study ranged from $\$ 1.00$ to $\$ 1000$, and the delay values ranged from 1 week to 25 years, with a total of 8 different delays. Lastly, the varying monetary values were presented in ascending and descending order in the present study, whereas they were presented once in random order within each condition (gain/loss) in Löckenhoff and colleagues' study. In summary, although the delay discounting procedure used in the present study involved hypothetical, rather than real monetary amounts, the procedure in this study produced data that were considerably more detailed than the procedure used by Löckenhoff and her colleagues.

In addition to discounting delayed losses more than older adults, younger adults also discounted delayed gains more than older adults in the present study, although this age difference was not significant. Green and colleagues (1994), who used a delay discounting procedure with the same parameters (i.e., dollar values, delay values, ascending and descending phases) as the one in the present study and found that younger adults discounted delayed gains significantly more steeply than older adults. Thus, although the direction of age differences in present study was in-line with the findings of Green and colleagues, the age differences in discounting of delayed gains in the present study did not reach statistical significance as it did in Green and colleagues' study. One interpretation of this finding is that the removal of nonsystematic delay discounting data in the present study may have resulted in insufficient power to detect an age difference. However, the findings presented by Green and colleagues were based on fewer younger and older adult participants than the findings of the present study (even after the removal of nonsystematic data from the present study). Moreover, Green and colleagues assessed 
discounting of delayed gains, but not of delayed losses. Thus, the nature of adult age differences in the discounting of delayed gains and losses requires further exploration.

Delay discounting procedures inherently require participants to consider the future (i.e., they involve considering gains or losses that will occur at some point in the future). The way in which people perceive the future has been shown to vary systematically with age—specifically, older adults typically have a more limited future time perspective than younger adults (FTP; Lang \& Carstensen, 2002). Thus, FTP is a relevant construct to consider in relation to delay discounting; particularly when age differences in delay discounting are being assessed. Furthermore, because limited FTP may be related to older adults' tendency to maximize positive emotions (Carstensen, 2006; Carstensen et al., 1999), FTP may relate to older adults' greater attention to positive — rather than negative—information (i.e., the "positivity effect; Carstensen \& Mikels, 2005). The "positivity effect" experienced by older adults may lead them to view monetary gains and losses differently than younger adults—-who tend to pay greater attention to negative, rather than positive information (i.e., the "negativity bias"; Baumeister et al., 2001; Rozein \& Ro; Baumeister et al., 2001; Rozin \& Royzman, 2001).

In the present study, FTP was positively related to Tradeoff Loss Aversion Task Scores, but inversely related to Delay Discounting Loss Aversion Scores. That is, people who perceived their future to be more expansive were more averse to monetary losses on the Tradeoff Loss Aversion Task. Alternately, people who perceived their future to be more expansive were less averse to monetary losses on the Delay Discounting Loss Aversion Task. Moreover, when analyses were conducted separately for each age group, Delay Discounting Loss Aversion Scores were inversely related to FTP for younger, but not for older adults. Tradeoff Loss Aversion Task Scores were not related to FTP when either age group was assessed independently. 
However, because people who were more loss averse, as indicted by Delay Discounting Loss Aversion Scores, also reported greater difficulty controlling spending on the Spendthrift Tightwad Scale (Rick et al., 2008), the validity of the Delay Discounting Loss Aversion Score is in doubt. Thus, relations between delay discounting of gains and losses and their respective relations with FTP offer opportunities for more clear interpretations. Similar to the relations between age and discounting of delayed gains and losses, FTP was not related to discounting of delayed gains, but was positively related to discounting of delayed losses; people who perceived their future as more expansive discounted delayed losses more than people who perceived their future as more limited. Put differently, people with more expansive FTP chose smaller immediate losses more frequently than larger delayed losses. One possible interpretation of this is that people with more expansive FTP may recognize that they will likely be alive when a delayed loss occurs, making a smaller immediate loss preferable to a larger delayed loss. On the other hand, people with limited FTP may assume that they will not be alive when the delayed loss occurs; thus making it preferable to an immediate loss, even if the immediate loss is smaller.

Taken together, the findings on the relations between age, FTP, and delay discounting of gains and losses suggest that younger adults have more expansive FTP than older adults and prefer smaller, immediate losses over larger delayed losses more than older adults. These findings can be interpreted within a framework proposed by Metcalfe and Mischel (1999), who describe delay discounting as involving a "cool" system, based on deliberative reasoning and emotional neutrality, and a "hot" system, based on emotional reactivity and impulsivity. Older adults report more effective emotion regulation abilities (Kessler \& Staudinger, 2009), are more accurate in reasoning about gains and losses (Wood et al., 2005), are less likely to experience declines in positive emotions when faced with a series of emotionally-challenging choices 
(Löckenhoff \& Carstensen, 2008), and exhibit less risk seeking in response to monetary loss cues (Mikels \& Reed, 2009). Furthermore, research suggests that older adults are less likely to experience negative affect when anticipating monetary losses, and are more accurate in predicting future affective responses to gains and loss than younger adults (Nielsen, Knutson \& Carstensen, 2008). These findings suggest that older adults may be more likely than younger adults to anticipate that a given outcome will have an approximately equal effect on their mood, regardless of whether the outcome is immediate or delayed (Löckenhoff et al., 2011). Moreover, these characteristics would suggest that older adults may be more likely than younger adults to utilize the "cool" system outlined by Metcalfe and Mischel when choosing between immediate and delayed gains and losses; suggesting a decrease in discounting of delayed outcomes with age (Löckenhoff et al.). Indeed, older adults discounted delayed gains and losses less than younger adults in this study (although only the age difference in discounting of delayed losses was significant; see results of Research Question 3).

However, age-related increases in attention to positive versus negative information and to gains versus losses (Carstensen \& Mikels, 2005; Samanez-Larkin et al., 2007) suggest that agerelated reductions in the discounting of delayed gains may be more prominent than reductions in the discounting of delayed losses (Löckenhoff et al., 2011). The findings of the present study suggest that younger adults discount delayed losses, but not gains, more than older adults. However, because variables related to affect and emotion regulation were not assessed in the present study, further research is needed to clarify the interrelations of affect, age, and discounting of delayed gains and losses.

\section{Implications, Limitations, and Future Directions}


Implications. Although the implications of demonstrating the sunk-cost fallacy were relatively trivial in the tasks that involved both real and hypothetical investments in the present study (i.e., spending a few dollars, investing time in a hobby or a boring movie), some prior research has suggested that the influence of sunk costs may be used for practical purposes that may have more meaningful implications. For instance, deposit contracts involve having people pay a particular amount before attempting to reach a goal--the investment is recouped if, and only if, the goal is attained — otherwise the investment is lost. Deposit contracts have been shown to be effective at influencing health-related behaviors such as abstinence from smoking (Dallery, Meredith, \& Glenn, 2008) and weight loss (Volpp et al., 2008). Behavior that is influenced by deposit contracts is, of course, different than behavior influenced by the sunk-cost fallacy because the person who has invested has a guaranteed opportunity to recoup their initial investment when investment contracts are employed. Nevertheless, the effectiveness of deposit contracts shows that monetary investments and loss aversion can be used to influence behavior for practical purposes. Similarly, research showing that younger adults follow costly advice more closely than free advice (Gino, 2008), suggests that accurate and helpful advice—such as health advice from a doctor-is more likely to be followed if it is paid for, than if it is free.

The findings of the present study, combined with prior research (e.g., Dallery et al, 2008; Gino, 2008) suggest several implications. First, because older adults demonstrated the sunk-cost fallacy less frequently and made normatively correct decisions more frequently than younger adults when hypothetical sunk costs were at stake, and there was no difference in how closely older adults followed more expensive advice compared to less expensive advice, the utility of sunk costs for practical purposes (i.e., deposit contracts, adherence to health advice) may be limited primarily to younger adults. 
Second, older adults who demonstrate the sunk-cost fallacy appear to engage in less overinvestment than younger adults who demonstrate the fallacy when hypothetical investments are at stake. Thus, examples of the sunk-cost fallacy demonstrated by older adults are generally not as "severe" as those demonstrated by younger adults. Even when they do demonstrate the sunk-cost fallacy, older adults are more likely to terminate continued investment at an earlier time or at a lower monetary amount than younger adults, who tend to continue overinvesting to a greater extent following a sunk cost (Garland, 1990; Strough et al., 2008). Prior work by Heath (1995; study 1) suggests that younger adults may be influenced to avoid overinvestment following sunk costs if they are given adequate information about the amount of the expected return on an initial investment (no information was provided regarding expected returns in the Sunk-Cost Fallacy Vignette Questionnaire used in the present study). Younger adults might not have overinvested as much in the present study if they had been given information on the expected returns related to the scenarios in the Sunk-Cost Fallacy Vignette Questionnaire.

Third, because younger adults in the present study generally did not choose to purchase advice when it was available to them during the Calorie Estimation Task, younger adults may be less likely than older adults to voluntarily make small monetary investments. Thus, when real money is involved, younger adults may preemptively circumvent the sunk-cost fallacy by avoiding the initial investment that is a prerequisite to the fallacy. The age difference in advice purchasing observed in the present study underscores the importance of testing for age differences in the ecological validity of self-report measures of the sunk-cost fallacy. Specifically, many studies investigating the sunk-cost fallacy among younger adults utilized hypothetical sunk costs that were not chosen by the research participants (i.e.; participants were told to imagine that they had already made hypothetical initial investments; e.g. Arkes \& Hutzel, 
2000; Bruine de Bruin et al., 2007; Strough et al., 2008). Although the age differences in demonstration of the sunk-cost fallacy on the self-report measure in the present study are consistent with prior research (i.e., Bruine de Bruin et al.; Strough et al.), it remains unclear to what extent younger adults would choose to make such initial investments, thus creating "realworld" sunk cost scenarios analogous to those described in hypothetical questionnaires.

Fourth, the age differences in discounting of delayed losses observed in the present study, and the corresponding positive relation between discounting of delayed losses and FTP, underscore the importance of considering both age and FTP when interpreting findings based on delay discounting procedures. Because perceptions of the future are an inherent component of all delay discounting procedures, FTP is a particularly relevant construct to include in research involving delay discounting procedures.

Limitations. There are several limitations of the present study that should be considered. First, because of the age differences in advice purchasing during the Calorie Estimation Task, I was unable to test for age differences in demonstration of the sunk-cost fallacy using this measure. Thus, it remains unclear whether the age differences in demonstration of the sunk-cost fallacy observed in this and other studies (e.g., Strough et al., 2008; 2011b) occur when people choose to make investments of real money. Moreover, it remains unclear whether loss aversion might serve as a mediator of age differences in demonstration of the sunk-cost fallacy when real monetary sunk costs are at stake.

Second, a substantial number of older adults' WOA Scores were ill-defined in that they exceeded 1, indicating that their final calorie estimate in a given condition was not a value between their initial estimate and the advice value. For instance, if a participant's initial estimate 
was 100 calories, the advice estimate was 150 calories, and the participant's final estimate was 175 calories, the WOA Score would have been 1.5. In the analyses reported in this study involving WOA Scores, ill-defined WOA Scores that exceeded 1 were recoded to 1 (following the approach described by Gino, 2008). This approach made it so that participants whose final estimate exceeded the advice value appeared to be following advice closely, although the extent to which the participant actually followed advice in such scenarios was unclear. Additionally, Gino reported a lower percentage of ill-defined WOA Scores that exceeded 1 than were observed in this study (3\% in study 1 of Gino, 2008 - compared to $31 \%$ in the current study). The reason for the difference in the number of ill-defined WOA Scores between this study and Gino's is unclear. However, there is one notable difference between this study and Gino's that might be partly responsible for the number of ill-defined WOA Scores in this study. Specifically, the present study included older adults, whereas Gino's study did not. It is possible that older adults responded to Calorie Estimation Task and the advice differently than younger adults in a manner that produced more ill-defined WOA Scores in this study, compared to Gino's study. However, it is impossible to know whether younger adults in this study would have produced more illdefined WOA Scores, had they purchased advice more frequently (and thus received more WOA Scores). Thus, it is impossible to determine, based on the data of the present study, whether the inclusion of older adults was responsible for the relatively large number of ill-defined WOA Scores in this study.

To provide assessments of advice following and demonstration of the sunk-cost fallacy based on advice following that were not subject to the large percentage of ill-defined WOA Scores, the Advice Adherence Scores and Total Advice Adherence Sunk-Cost Fallacy Scores were created. The Advice Adherence Scores indicated the degree to which a participant's final 
estimate adhered to, or deviated from, the advice value, relative to their initial estimate; even in instances in which the final estimate was not a value between the initial estimate and the advice value. Put differently, the Advice Adherence Scores represented whether a participant's final estimate was closer to, or further away from the advice value, relative to their initial estimate. Additionally, Total Advice Adherence Sunk-Cost Fallacy Scores showed whether a participant demonstrated the sunk-cost fallacy by making a final estimate in the large price of advice condition that was closer to the advice value (relative to their initial estimate) than in the small price of advice condition.

The creation of Advice Adherence Scores and Total Advice Adherence Sunk-Cost Fallacy Scores provided an alternative means of addressing Exploratory Questions 5, 6, 7, and 13; each of which involved either WOA Scores or Calorie Estimate Sunk-Cost Fallacy Scores, which were based on WOA Scores (and were therefore affected by ill-defined WOA Scores). The results of the analyses that addressed Exploratory Questions 5, 6, 7 and 13 were all the same in terms of significance, regardless of whether Advice Adherence Scores were used in place of WOA Scores, or whether Total Advice Adherence Sunk-Cost Fallacy Scores were used in place of Calorie Estimate Sunk-Cost Fallacy Scores.

Third, although adapted from existing measures (i.e., Green et al., 1994; Fennema \& Van Assen, 1999), the Delay Discounting Loss Aversion Task and the Tradeoff Loss Aversion Task were both novel measures of loss aversion. Although loss aversion is a commonly cited explanation for a variety of behaviors, there are relatively few existing measures of loss aversion (Abdellaoui, Bleichrodt, \& Paraschiv, 2007), and the measures used in this study should be assessed in conjunction with other measures to verify their validity. 
Fourth, a substantial percentage of delay discounting data was nonsystematic and was therefore removed. Prior research has shown that approximately $2 \%$ of participants who complete delay discounting procedures similar to those used in this study violate criterion 1 , and approximately $5 \%$ of participants violate criterion 2 used to identify nonsystematic data (Johnson $\&$ Bickel, 2008). These rates are considerably lower than the $31 \%$ of participants who violated criterion 1 more than once and the $41 \%$ of participants who violated criterion 2 in the present study. However, the data sets that Johnson and Bickel used were based on delay discounting procedures that only involved a gains condition (whereas the present study involved both gains and losses conditions, effectively doubling the number of data points, and thus the opportunities for a participant's data to be removed due to nonsystematic data). Additionally, the data sets used by Johnson and Bickel were based on delay discounting procedures that involved 7 delays, as opposed to the 8 delays used in the present study (again, making it more likely that nonsystematic data would occur in the present study, compared to those assessed by Johnson and Bickel). However, even if one were to make the liberal assumption that the procedures used in this study presented three times the opportunity for participants to produce nonsystematic data compared to the studies assessed by Johnson and Bickel, one would still only expect that approximately $6 \%$ of participants would violate criterion 1 , and $15 \%$ of participants would violate criterion 2.

Johnson and Bickel (2008) suggest that nonsystematic data is indicative of a lack of understanding of the task among participants. Additionally, Johnson and Bickel suggest nonsystematic data may show that the delay discounting procedure did not encourage careful responding, leading participants to repeatedly respond on the same button without paying attention to the monetary or delay values. The present study included comprehensive instructions 
for participants, and participants were reminded that they could ask the research assistant to provide guidance at any time if they didn't understand the task. Additionally, participants viewed an example of the delay discounting task prior to beginning. However, participants in the present study were not given the opportunity to engage in a "practice" delay discounting session after reading the instructions but before beginning the "real" session. Thus, participants may have become confused after beginning the delay discounting task, at which point they may have assumed that it was "too late" to ask the research assistant to clarify the task for them. Such confusion among participants may be responsible for the relatively high rates of nonsystematic data. However, because the initial sample that completed the delay discounting procedure in the present study was relatively large (compared to the original study on which the delay discounting procedure in this study was based; i.e., Green et al., 1994), there were still a sufficient number of cases remaining after the removal of nonsystematic data to address a number of the research questions.

Fifth, the delay discounting procedure in the present study used one constant delayed monetary value (i.e., \$1000). This amount was chosen based on prior research of age differences of delay discounting of gains and losses (Green et al., 1994). However, prior studies have shown that rates of discounting differ based on the magnitude of the delayed reward. Specifically, as the delayed reward is increased, rates of discounting tend to decrease (i.e., as the delayed reward increases in magnitude, people tend to choose the delayed reward over the smaller immediate reward more frequently; Green, Myerson \& Ostaszewski, 1999). However, the effects of the magnitude of the delayed outcome are less clear when losses are at stake — such effects tend to be smaller and less reliable than the effect of the magnitude of delayed gains (Estle, Green, 
Myerson \& Holt, 2006). Thus, the data from the present study does not address the effects of the magnitude of the delayed gain or loss.

Sixth,, low statistical power was likely an issue for several analyses, particularly with respect to Research Question 5, and Exploratory Questions 4, 5, 6, 7, and 9. The analyses involved in Research Question 5 (which tested Delay Discounting Loss Aversion Scores as a mediator of the relation between age group and Sunk-Cost Fallacy Vignette Score) utilized methods that have been cited as having improved power over the traditional Baron and Kenny (1986) approach (i.e., Sobel tests and nonparametric bootstrapping; Preacher \& Hayes, 2004; Shrout \& Bolger, 2002; Sobel, 1982). Nevertheless, these analyses were based on limited data due to the removal of nonsystematic delay discounting data. Similarly, insufficient power due to the removal of nonsystematic delay discounting data may explain the non-significant relations between Delay Discounting Loss Aversion Task Scores and advice purchasing (Exploratory Question 4), Calorie Estimate Sunk-Cost Fallacy Scores and Total Advice Adherence Sunk-Cost Fallacy Scores for older adults(Exploratory Question 5), and Tradeoff Loss Aversion Task Scores (Exploratory Question 10). Insufficient power due to low rates of advice purchasing (and thus a low proportion of participants receiving Calorie Estimate Sunk-Cost Fallacy Scores and Total Advice Adherence Sunk-Cost Fallacy Scores), may also explain why there was not a statistically significant difference in how heavily older adults weighted and adhered to advice from the small and large price of advice conditions, as well as the non-significant relations between Sunk-cost Fallacy Vignette Scores and both the Calorie Estimate Sunk-Cost Fallacy Scores, and Total Advice Adherence Sunk-Cost Fallacy Scores among older adults (Exploratory Question 6). Likewise, low power due to low rates of advice purchasing may also explain why there was not a significant difference in how older adults perceived advice from the small and the 
large price of advice conditions, and the non-significant relation between Quality of Advice Score from the large price of advice condition and both the Calorie Estimate Sunk-Cost Fallacy Scores and the Total Advice Adherence Sunk-Cost Fallacy Scores (Exploratory Question 7; Cohen, 1998; Faul et al., 2009; Field, 2009).

Seventh, although participants in the present study were informed that the advice originated from an expert, participants' perceptions of the quality of the advice were not assessed prior to their purchase of the advice. Moreover, perceptions of the quality of advice were only assessed for participants who chose to purchase advice. Thus, it remains unclear whether people who purchased advice had different perceptions of the advice or the potential outcomes of purchasing advice than people who chose not to purchase advice. Moreover, it remains unclear whether peoples' perceptions of advice prior to purchasing advice related to their likelihood of purchasing the advice.

Eighth, older adults took significantly longer than younger adults to complete the procedure in the present study. This may be interpreted in a number of ways. Older adults may have been slower than younger adults in their information processing and reaction times when completing the procedure of the present study; prior research has noted the inverse relation between age and information processing (e.g; Salthouse \& Somberg, 1982). Moreover, the older adult participants may have been less familiar with computer hardware and software than the younger adults, which may have related to more careful mouse clicking and data entry, and thus longer completion times. Additionally, older adults' longer completion time may have been indicative of an attempt to complete the tasks as accurately as possible, whereas younger adults' shorter completion times may have been indicative of an attempt to complete the task as quickly 
as possible — such age differences in motivations may have also been reflected in older adults' greater likelihood of purchasing advice, relative to younger adults.

Ninth, sex was not equally disbursed across age groups (68\% of the younger adults were women, compared to $56 \%$ of older adults). However, there were no significant differences between males and females in rates of advice purchasing - either within the respective age groups, or when the age groups were combined. Moreover, sex was included as a covariate in the analyses described in the results section; results were the same in terms of direction and significance regardless of whether sex was or was not included.

Tenth, the form of continued investments in the Calorie Estimation Task that defined demonstration of the sunk-cost fallacy differed conceptually from other examples of continued investment from prior studies. Previous studies have defined continued investment in terms of money or time (e.g., Strough et al., 2011b). Some studies have defined continued investment that leads to the sunk cost fallacy in terms of a combination of time and effort-attending more theater performances after paying more money for a season ticket (compared to when the ticked price was discounted), for instance (Arkes \& Blumer, 1985; see Strough et al., 2011a for a review). However, in the present study, the continued investment that lead to the sunk-cost fallacy was defined in terms of how much weight participants gave to advice (i.e., how closely they followed advice).

Traditional definitions of the sunk-cost fallacy simply define the fallacy in terms of continued investment of some resource following an initial investment (Arkes \& Ayton, 1999), without necessarily specifying whether there are limitations in what constitutes an "investment" or a "resource". In the present study, weighting advice can be viewed as continued investment in 
the sense that the goal of the task was to make accurate estimates, thereby earning more money, and following advice would presumably increase the likelihood of making an accurate estimate. Put differently, following advice during the Calorie Estimation Task can be viewed as an investment because it involved devotion to a course of action intended to produce future benefits. Thus, in the Calorie Estimation Task, following more expensive advice more closely than less expensive advice would be an example of the sunk-cost fallacy.

Eleventh, it is possible that the estimates during the Calorie Estimation Task provided by participants who purchased advice were controlled by variables other than the price of advice. For example, a participant may have assumed that the optimal strategy during the Calorie Estimation Task was to make an estimate in the second phase of a price of advice condition that was very different than their initial estimate from the first phase. Because each participant's knowledge of food was constant, the actual caloric content of the analogous food item pairs was constant, the advice was constant, and the advice was presented as an estimate made by an expert in the present study, the optimal strategy for participants was to assume that the expert would have a reasonably accurate knowledge of calories, and that it would therefore be prudent to follow the advice to some degree. The primary question of interest was whether the participant followed the advice more closely when the advice was more expensive than when it was less expensive. However, if participants assumed that following advice had nothing to do with an increased likelihood of making accurate estimates, their decisions may have deviated substantially from what might considered the optimal strategy of following advice.

Twelfth, the Calorie Estimation Task was based on a procedure used by Gino (2008, study 1), but the task was changed and adapted for the present study (i.e., in this study, participants estimated calories instead of historical dates, advice originated from an expert rather 
than another participant, and the prices of advice and potential monetary returns were different) . There are several potential implications of these changes that should be considered. First, it is possible that participants in the present study were able to estimate calories more accurately than participants in Gino's (2008) study were able to estimate historic dates. Such an effect might have influenced rates of advice purchasing and/or estimate accuracy. Unfortunately, Gino did not provide information regarding the accuracy of participants' estimates. However, the analyses of Exploratory Questions 1 and 2 suggest that neither estimate accuracy, nor confidence was related to advice purchasing in the present study. Moreover, the topic of the advice may have influenced rates of advice purchasing; people may be more willing to pay for advice related to historic dates than caloric values. Second, because the initial show-up fee was less than, and the amount earned for accurate estimates was more than, Gino's study, participants may have had less motivation to show up for the study, and more motivation to make accurate estimates, respectively (compared to participants in Gino's study). Participant recruitment occurred at a relatively even pace during data collection, suggesting that the $\$ 5$ show-up fee was sufficiently motivating for participants to attend data collection sessions. However, it is unknown whether the smaller show-up fee attracted younger adult participants who were different than Gino's on a variable that might be related to their decisions on the Calorie Estimation Task. It is possible that young adults who were willing to accept the $\$ 5.00$ show up fee in this study were less likely to purchase advice than younger adults who attended Gino's study and received a $\$ 10.00$ show up fee. Third, because advice cost money in both conditions of the present study (as opposed to free advice and \$4 advice in the respective conditions in Gino's study), participants in Gino's study may have been more likely to purchase advice; particularly participants involved in the estimate sequence in which the free advice condition preceded the $\$ 4.00$ advice condition. Indeed, participants in 
Gino's study purchased advice more frequently when free advice was offered to them in the first condition, and paid advice in the second condition. However, rates of advice purchasing were still substantially higher in Gino's study than in the present study, even among Gino's participants who entered the $\$ 4.00$ advice condition before the free advice condition (46\% of Gino's participants purchased advice when conditions were arranged in this sequence). Lastly, because advice in the present study originated from an expert, and advice in Gino's study originated from another participant, the motivational factors associated with purchasing and following advice may have been different between studies. In particular, expert advice is presumably more useful than non-expert advice, which would suggest that people might be more willing to purchase expert rather than non-expert advice (which was not the case when comparing this study with Gino’s).

Lastly, the sample of participants used in the present study was predominantly White/Caucasian (see Table 2), and was a convenience sample. Thus, it remains unclear whether the results of the present study generalized to individuals of other races and ethnicities.

Future directions. Several directions for future research are proposed. First, given the age differences in advice purchasing that were observed in the present study, future studies should assess differences between younger and older adults' willingness to make initial investments that are prerequisites to the sunk-cost fallacy. More specifically, studies should focus on younger and older adults' willingness to invest resources of time, effort, and money in scenarios that are similar to those described in self-report measures that involve hypothetical sunk costs (e.g., Bruine de Bruin et al., 2007; Strough et al., 2011b). 
Second, the behavioral measure of the sunk-cost fallacy employed in this study (and adapted from Gino, 2008), should be refined, and new measures should be developed. For instance, in the present study, the Calorie Estimation Task involved four phases so that Weight of Advice Scores could be computed. A simplified version of this task might only employ two phases (one with more expensive advice, one with less expensive advice). Although this would not allow one to calculate Weight of Advice Scores (as in the present study and as in Gino's study) or Advice Adherence Scores (as in the present study), one could assess whether participants demonstrated the sunk-cost fallacy based on whether their estimates corresponded to the advice more or less closely as a function of the cost of the advice. Specifically, if a participant's estimate resembled expensive advice more closely than inexpensive advice, they would be demonstrating the sunk-cost fallacy. Moreover, such a procedure would be simpler than the one used in the present study, and wouldn't require the extensive set of instructions used in the present study. Other behavioral measures involving real monetary sunk costs (similar to the task involving the purchase of real estate in the Monopoly board game using real money used by Heath, 1995), could be explored, as well as behavioral analogues of existing self-report measures (such as those used by Bruin de Bruine et al., 2007 and Strough et al., 2008).

Third, the relation between the sunk-cost fallacy and peoples' perceptions of the object of investment should be investigated. In the present study, people who purchased advice responded to a measure of their perceived quality of the advice, but not until after they had received it. Future research might address the question of whether people who perceive a good or resource as being of a higher value are more likely to make investments (i.e., sunk costs) to obtain the good or resource. For example, any future studies that use the Calorie Estimation Task from this study, the historical date estimation task from Gino's (2008) study, or an adaptation of either, may 
consider assessing participants' perceptions of the quality of, and their need for advice before participants are able to purchase the advice (rather than after they have purchased it).

Fourth, in light of the age differences in advice purchasing observed in the present study, future research should investigate the circumstances under which younger and older adults differ in their likelihood of making the initial investments that are prerequisites to the sunk-cost fallacy. For instance, future studies may focus on whether younger and older adults differ in how likely they are to make the investments described in the hypothetical self-report measures used in prior studies - such as paying money to view a show on pay-per-view television (i.e., Strough et al., 2008). Researchers may investigate such questions by studying the purchasing behavior of younger and older adults using real money, or with self-report questionnaires asking participants to indicate which types of sunk cost investments they would be likely to make.

Fifth, as mentioned in the limitations section above, the definition of the sunk-cost fallacy from the Calorie Estimation Task was based on a type of investment (i.e., advice following) that was conceptually different from those that have been studied in most previous investigations of the sunk-cost fallacy. Thus, it may be useful for researchers to work toward building a consensus for whether there are limitations on what types of decisions and behaviors constitute an investment, and if so, what those limitations are.

Sixth, also as mentioned in the limitations section above, if participants assumed that following advice in the Calorie Estimation Task had nothing to do with an increased likelihood of making accurate estimates, their decisions may have deviated substantially from what might considered the optimal strategy of following advice. Additionally, it is unknown whether factors other than the cost of advice influenced how closely participants followed advice. Therefore, it 
may be beneficial for future studies to investigate participants' perceptions of what constitutes an optimal strategy in sunk cost scenarios, provide a more direct description of the optimal strategy to participants before they complete a sunk cost task, or both. Additionally, it may be useful to assess whether, and to what degree, participants are basing their decisions on the amount or existence of the initial investment (i.e., the sunk cost). Initial studies regarding participants' decision making goals and their attention to the amount and presence of sunk costs in sunk cost scenarios have been completed recently (e.g., Strough et al., 2011b).

Seventh, future studies may benefit from assessing how closely people follow lessversus more-expensive advice, irrespective of the expertise of the person providing the advice. Gino's (2008) study involved a procedure in which the advice was provided by a person who was presumably as well-informed about the topic at hand as the participant. This procedure would allow researchers to assess the influence of the price of advice independent from the influence of participants' perceptions of the expertise of the person providing advice.

Eight, future research might investigate the types of advice people are willing to pay for, and variables related to why people might be more willing to pay for some types of advice compared to others. For instance, research investigating why participants might be more willing to pay for advice pertaining to historic dates than calorie values could help to explain the differences between this and Gino's (2008) study in rates of advice purchasing. Moreover, future studies may investigate how the amount of money that participants receive as initial show-up fees might relate to their subsequent likelihood of purchasing advice and/or their decisions about sunk cost scenarios. 
Lastly, although loss aversion did not mediate the relation between age and demonstration of the sunk-cost fallacy in the present study, other potential mediators may be investigated. For instance, people may demonstrate the sunk-cost fallacy in order to avoid acknowledging that the initial investment was a bad decision (Fox \& Staw, 1979), or to avoid being perceived as "wasting" the initial investment (Arkes, 1996). Younger adults' greater susceptibility to the negativity bias (Baumeister et al., 2001) might make them more averse to negative self-perceptions related to making poor investment decisions or "wasting" sunk costs than older adults. If younger adults are more concerned than older adults with the negative information associated with acknowledging bad investment decisions and/or being perceived as wasteful, these perceptions might mediate age differences in the sunk-cost fallacy. Furthermore, prior research (Strough et al., 2011b) has shown that the goals that younger and older describe in relation to sunk cost scenarios differ; younger adults tend to focus more on the presence or absence of the sunk cost when providing explanations for how they respond to sunk cost scenarios. The focus on sunk costs when describing decision-making goals has been shown to mediate the relation between age and the sunk-cost fallacy (Strough et al.). Thus, the degree to which people focus on the presence or absence of sunk costs when formulating decision-making goals should be further investigated as a mechanism that may account for age differences in demonstration of the sunk-cost fallacy.

\section{Conclusions}

The sunk-cost fallacy is a decision-making bias that can occur in a variety of contexts where money, time, or effort has been invested, and which can have relatively trivial, or serious consequences. Age differences in demonstration of the sunk-cost fallacy and normatively correct decisions appear to be robust phenomena; older adults demonstrate the sunk-cost fallacy less 
frequently, overinvest less, and make normatively correct decisions more frequently, than younger adults. Preliminary evidence suggests that when real money is at stake, younger adults are less likely than older adults to make small monetary investments that are prerequisites to the sunk-cost fallacy, and older adults do not demonstrate the sunk-cost fallacy by following more expensive advice any more closely than less expensive advice that they have paid for.

Additionally, preliminary evidence suggests that older adults are less averse to monetary losses than younger adults, although loss aversion does not mediate age differences in demonstration of the sunk-cost fallacy. Future research should continue to investigate age differences in demonstration of the sunk-cost fallacy when real, rather than hypothetical sunk costs have been invested, while further investigating potential mediators of the relation between age and the sunk-cost fallacy. 


\section{References}

Abdellaoui, M., Bleichrodt, H., \& Paraschiv, C. (2007). Loss aversion under prospect theory: A parameter-free measurement. Management Science, 54, 1659-1674.

Adams, G., \& Rau, B. (2004). Job seeking among retirees seeking bridge employment. Personnel Psychology, 57, 719-744.

Arkes, H.R. (1996). The psychology of waste. Journal of Behavioral Decision Making, 9, 213214.

Arkes, H. R., \& Ayton, P. (1999). The sunk cost and concorde effects: Are humans less Rational than lower animals? Psychological Bulletin, 125, 591-600.

Arkes, H.R., \& Blumer (1985). The psychology of sunk cost. Organizational Behavior and Human Decision Processes, 35, 124-140.

Arkes, H.R., \& Hutzel, L. (2000). The role of probability of success estimates in the sunk cost effect. Journal of Behavioral Decision Making, 13, 295-306.

Baker, F., Johnson, M.W., \& Bickel, W.K. (2003). Delay discounting in current and never-before cigarette smokers: Similarities and differences across commodity, sign, and magnitude. Journal of Abnormal Psychology, 112, 382-392.

Baron, R.M., \& Kenny, D.A. (1986). The moderator-mediator variable distinction in social psychological research: Conceptual, strategic, and statistical considerations. Journal of Personality and Social Psychology, 51, 1173-1182. 
Bassett, W.F., Flemming, M.J., \& Rodrigues, A.P. (1998). How workers use 401(k) plans: The participation, contribution, and withdrawal decisions. National Tax Journal, 51, 263-289.

Baumeister, R.F., Bratslavsky, E., Finkenauer, C., \& Vohs, K.D. (2001). Bad is stronger than good. Review of General Psychology, 5, 323-370.

Beeler, J.D., \& Hunton, J.E. (1997). The influence of compensation method and disclosure level on information search strategy and escalation of commitment. Journal of Behavioral Decision Making, 10, 77-91.

Benzion, U., Rapoport, A., \& Yagil, J. (1989). Discount rates inferred from decisions: An experimental study. Management Science, 35, 270-284.

Blomquist, G. C., \& Whitehead, J. C. (1998). Resource quality information and validity of willingness to pay in contingent valuation. Resource and Energy Economics, 20, 179196.

Blumenschein, K., Johannesson, M., Blomquist, G. C., Liljas, B., \& O'Conor, R. M. (1998). Experimental results on expressed certainty and hypothetical bias in contingent valuation. Southern Economic Journal, 65(1), 169-177.

Blumenschein, K., Johannesson, M., Yokoyama, K. K., \& Freeman, P. R. (2001). Hypothetical versus real willingness to pay in the health care sector: results from a field experiment. Journal of Health Economics, 20, 441-457.

Bornstein, B.H., \& Chapman, G.B. (1995). Learning lessons from sunk costs. Journal of Experimental Psychology: Applied, 1, 251-269.

Botelho, A., \& Pinto, L.C. (2001). Hypothetical, real, and predicted real willingness to pay in open-ended surveys: experimental results. Applied Economics Letters, 2002(9) 993-996. 
Brockner, J. (1992). The escalation of commitment to a failing course of action: Toward theoretical progress. Academy of Management Review, 17, 39-61.

Bruine de Bruin, W., Parker, A.M., \& Fischhoff, B. (2007). Individual differences in adult decision-making competence. Journal of Personality and Social Psychology, 92, 938956.

Bushong, B., King, L. M., Camerer, C. F., \& Rangel, A. (2010) Pavlovian processes in consumer choice: the physical presence of a good increases willingness-to-pay. American Economic Review, 100, 1-18.

Campbell, D. T., \& Fiske, D. W. (1959). Convergent and discriminant validation by the multitrait-multimethod matrix. Psychological Bulletin, 56, 81-105.

Carstensen, L. L. (2006). The influence of a sense of time on human development. Science, 312, 1913-1915.

Carstensen, L., \& Lang, F. R. (1996). Future time perspective scale. Unpublished manuscript, Stanford University, Stanford: CA.

Carstensen, L. L., \& Mikels, J.A. (2005). At the intersection of emotion and cognition: Aging and the positively effect. Current Directions in Psychological Science, 14, 117-121.

Carstensen, L. L., \& Hartel, C. R. (Eds.). (2006). When I'm 64. Washington, DC: The National Academies Press.

Carstensen, L. L., Isaacowitz, D.M., \& Charles, S.T. (1999). Taking time seriously: A theory of socioemotional selectivity. American Psychologist, 54, 165-181.

Chao, L., Szrek, H., Pereira, N., \& Pauly, M. V. (2009). Time preference and its relationship with age, health, and survival probability. Judgment and Decision Making, 4(1), 1-19. 
Charles, S.T., Mather, M., \& Carstensen, L.L. (2003). Aging and emotional memory: The forgettable nature of negative images for older adults. Journal of Experimental Psychology: General, 132, 310-324.

Chen, Y., \& Sun, Y. (2003). Age differences in financial decision-making: Using simple heuristics. Educational Gerontology, 29, 627-635.

Cohen, J. (1998). Statistical power analysis for the behavioral sciences ( $2^{\text {nd }}$ ed.). Mahwah, NJ: Lawrence Erlbaum Associates

Coleman, M. D. (2010). Sunk cost and commitment to medical treatment. Current Psychology: Research \& Reviews, 29(2), 121-134. doi:10.1007/s12144-010-9077-7

Cummings, R. G., Harrison, G. W., \& Ruström, E. E. (1995). Homegrown values and hypothetical surveys: Is the dichotomous choice approach incentive-compatible? The American Economic Review, 85(1), 260-266.

Cunha, M., \& Caldieraro, F. (2009). Sunk-cost effects on purely behavioral investments. Cognitive Science, 33, 105-113.

Dallery, J., Meredith, S., \& Glenn, I.M. (2008). A deposit contract method to deliver abstinence reinforcement for cigarette smoking. Journal of Applied Behavior Analysis, 41, 609-615.

Denburg, N.L., Tranel, D., \& Bechara, T.A. (2005). The ability to decide advantageously Declines prematurely in some normal older persons. Neuropsychologia, 43, 1099-1106.

Estle, S. J., Green, L., Myerson, J., \& Holt, D. D. (2006). Differential effects of amount on temporal and probability discounting of gains and losses. Memory \& Cognition, 34, 914928. 
Fagerlin, A., Zikmund-Fisher, B. J., Ubel, P. A., Jankovic, A., Derry, H. A., \& Smith, D. M. (2007). Measuring numeracy without a math test: Development of the Subjective Numeracy Scale. Medical Decision Making, 27, 672-680.

Faul, F., Erdfelder, E., Buchner, A., \& Lang, A.-G. (2009). Statistical power analyses using G*Power 3.1: Tests for correlation and regression analyses. Behavior Research Methods, 41, 1149-1160.

Fennema, H., \& Van Assen, M. (1999). Measuring the utility of losses by means of the tradeoff method. Journal of Risk and Uncertainty, 17, 277-295.

Field, A. (2009). Discovering statistics using SPSS (3 $3^{\text {rd }}$ ed). Thousand Oaks, CA: Sage Publications Inc.

Fisher, R. A. (1921). On the 'probable error' of a coefficient of correlation deduced from a small sample. Metron, 1, 3-32.

Fox, F.V., \& Staw, B.M. (1979). The trapped administrator: Effects of job insecurity and policy Resistance upon commitment to a course of action. Administrative Science Quarterly, 24, 449-471.

Friedman, D., Pommerenke, K., Lukose, R., Milam, G., \& Huberman, B. A. (2007). Searching for the sunk cost fallacy. Experimental Economics, 10, 79-104.

Frisch, D. (1993). Reasons for framing effects. Organizational Behavior and Human Decision Processes, 54, 399-429.

Garland, H. (1990). Throwing good money after bad: The effect of sunk costs on the decision to escalate commitment to and ongoing project. Journal of Applied Psychology, 75, 728731. 
Garland, H., \& Newport, S. (1991). Effects of absolute and relative sunk costs on the decision to Persist with a course of action. Organization behavior and human design processes, 48, 55-69.

Genesove, D., \& Mayer, C. (2001). Loss aversion and seller behavior: Evidence from the housing market. The Quarterly Journal of Economics, 1233-1260.

Gino, F. (2008). Do we listen to advice just because we paid for it? The impact of advice cost on its use. Organization Behavior and Human Decision Processes, 107, 234-245.

Grace, T. A., \& Sawilowsky, S. S. (2009). Data error prevention and cleansing: A comprehensive guide for instructors of statistics and their students. Model Assisted Statistics and Applications, 4, 303-312. doi: 10.3233/MAS-2009-0140

Green, L., Fry, A.F., \& Myerson, J. (1994). Discounting of delayed rewards: A life-span comparison. Psychological Science, 5, 33-36.

Green, L, Myerson, J., \& Ostaszewski, P. (1999). Amount of reward had opposite effects on the discounting of delayed and probabilistic outcomes. Journal of Experimental Psychology: Learning, Memory, and Cognition, 25(2), 418-427.

Gigerenzer, G., Czerlinski, J., \& Martignon, L. (1999). How good are fast and frugal heuristics? In J. Shanteau, B. Mellers, \& D. Schum (Eds.), Decision science and technology: Reflections on the contributions of Ward Edwards (pp. 81-102). Norwell, MA: Kluwer Academic.

Goodwin, C. J. (2008). Research in Psychology: Methods and Design (5th ed.). Hoboken, NJ: John Wiley \& Sons.

Harrison, G. W., Lau, M. I., \& Williams, M. B. (2002). Estimating individual discount rates in Denmark: A field experiment. The American Economic Review, 92(5), 1606-1617. 
Heath, C. (1995). Escalation and de-escalation of commitment in response to sunk costs: The role of budgeting in mental accounting. Organizational Behavior and Human Decision Processes, 62, 38-54.

Holden, S., \& VanDerhei, J. (2001). Contribution behavior of 401(k) plan participants. Investment Company Institute Perspective, 7, 1-20.

Johannesson, M., Liljas, B., \& Johansson, P. (1998). An experimental comparison of dichotomous choice contingent valuation questions and real purchase decisions. Applied Economics, 30, 643-647.

Johnson, M. W., \& Bickel, W. K. (2008). An algorithm for identifying nonsystematic delaydiscounting data. Experimental and Clinical Psychopharmacology, 16(3), 264-274. doi: 10.1037/1064-1297.16.3.264

Kahneman, D., \& Tversky, A. (1979). Prospect theory: An analysis of decision under risk. Econometrica, 47, 263-291.

Kahneman, D., Knetsch, J.L., \& Thaler, R.H. (1990). Experimental tests of endowment effect and the coase theorem. The Journal of Political Economy, 98, 1325-1348.

Keil, M., Truex II, D. P., \& Mixon, R. (1995). The effects of sunk cost and project completion on information technology project escalation. Transactions on Engineering Management, 42, 372-381.

Kessler, E., \& Staudinger, U. M. (2009). Affective experience in adulthood and old age: The role of affective arousal and perceived affect regulation. Psychology and Aging, 24, 349-362.

Klaczynski, P.A. (2001). Framing effects on adolescent task representations, analytic and heuristic processing, and decision making implications for the normative/descriptive gap. Applied Developmental Psychology, 22,289-309. 
Lang, F. R., \& Carstensen, L. L. (2002). Time counts: Future time perspective, goals, and social relationships. Psychology and Aging, 17(1), 125-139. doi:10.1037/0882-7974.17.1.125

Larrick, R.P, \& Soll, J.B. (2006). Intuitions about combining opinions: Misappreciation of the averaging principle. Management Science, 52(1), 111-127.

Larrick, R.P., Nisbett, R.E., \& Morgan, J.N. (1993). Who uses the cost-benefit rules of choice? Implications for the normative status of microeconomic theory. Organizational Behavior and Human Decision Processes, 56, 1-23.

Löckenhoff, C. E., \& Carstensen, L. L. (2008). Decision strategies in health care choices for self and others: Older but not younger adults make adjustments for the age of the decision target. Journal of Gerontology: Psychological Sciences, 63B, 106-109.

Löckenhoff, C. E., O'Donoghue, T., \& Dunning, D. (2011). Age differences in temporal discounting: The role of dispositional affect and anticipated emotions. Psychology and Aging, 26(2), 274-284. doi:10.1037/a0023280

Loomis, J., Brown, T., Lucero, B., \& Peterson, G. (1997). Evaluating the validity of the dichotomous choice question format in contingent valuation. Environmental and Resource Economics, 10, 109-123.

MacKinnon, D. P., Lockwood, C. M., Hoffman, J. M., West, S. G., \& Sheets, V. (2002). A comparison of methods to test mediation and other intervening variable effects. Psychological Methods, 7(1), 1-35.

Mather, M., \& Carstensen, L.L (2005). Aging and motivated cognition: The positivity effect in attention and memory. Trends in Cognitive Sciences, 9(10), 496-502. 
Mather, M., Canli, T., English, T., Whitfield, S., Wais, P., \& Ochsner, K., et al. (2004). Amygdala responses to emotionally valenced stimuli in older and younger adults. Psychological Science, 15(4), 259-263.

Metcalfe, J., \& Mischel, W. (1999). A hot/cool-system analysis of delay of gratification: Dynamics of willpower. Psychological Review, 106, 3-19.

Meyer, J. (2001). Age: 2000: Census 2000 brief. Washington, D.C.: U.S. Census Bureau. Mikels, J. A., \& Reed, A. E. (2009). Monetary losses do not loom large in later life: Age differences in the framing effect. The Journals of Gerontology: Series B: Psychological Sciences and Social Sciences, 64B(4), 457-460.

Molden, D. C., \& Hui, C. M. (2011). Promoting de-escalation of commitment: A regulatoryfocus perspective on sunk costs. Psychological Science, 22, 8-12.

Myerson, J., Green, L., \& Warusawitharana, M. (2001). Area under the curve as a measure of discounting. Journal of the Experimental Analysis of Behavior, 76, 235-243.

Navarro, A.D., \& Fantino, E. (2005). The sunk cost effect in pigeons and humans. Journal of the Experimental Analysis of Behavior, 83(1), 1-13.

Navarro, A.D, \& Fantino, E. (2009). The sunk-time effect: An exploration. Journal of Behavioral Decision Making, 22, 252-270.

Neill, H. R., Cummings, R. G., Ganderton, P. T., Harrison, G. W., \& McGuckin, T. (1994). Hypothetical surveys and real economic commitments. Land Economics, 70(2) 145-154.

Nielsen, L., Knutson, B., \& Carstensen, L. L. (2008). Affect dynamics, affective forecasting, and aging. Emotion, 8, 318-330.

Peters, E., Hess, T.M., Västfjäll, D., \& Auman,C. (2007). Adult age differences in dual information processes. Perspectives on Psychological Science, 2(1), 1-23. 
Preacher, K. J., \& Hayes, A. F. (2004). SPSS and SAS procedures for estimating indirect effects in simple mediation models. Behavior Research Methods, Instruments, \& Computers, 36, 717-731.

Rao, A. R., \& Monroe, K. B. (1989). The effect of price, brand name, and store name on buyers' perceptions of product quality: An integrative review. Journal of Marketing Research, 26, 351-357.

Read, D., \& Read, N. L. (2004). Time discounting over the lifespan. Organizational Behavior and Human Decision Processes, 94, 22-32.

Reimers, S., Maylor, E. A., Stewart, N., \& Chater, N. (2009). Associations between a one-shot delay discounting measure and age, income, education and real-world impulsive behavior. Personality and Individual Differences, 47(8), 973-978.

Reyna, V. F., \& Farley, F. (2006). Risk and rationality in adolescent decision making. Psychological Science in the Public Interest, 7, 1-44.

Rick, S. I., Cryder, C. E., \& Loewenstein, G. (2008). Tightwads and spendthrifts. Journal of Consumer Research, 34, 767-782.

Rozin, P., \& Rozyman, E. B. (2001). Negativity bias, negativity dominance, and contagion. Personality and Social Psychology Review, 5, 296-320.

Salthouse, T. A., \& Somberg, B. L. (1982). Isolating the age deficit in speed performance. Journal of Gerontology: Psychological Sciences, 37 (1), 59-63. 
Samanez-Larkin, G. R., Gibbs, S. B., Khanna, K., Nielsen, L., Carstensen, L. L., \& Knutson, B. (2007). Anticipation of monetary gain but not loss in healthy older adults. Natural Neuroscience, 10(6), 787-791.

Sidman, M. Tactics of scientific research. New York: Basic Books; 1960.

Shrout, P. E., \& Bolger, N. (2002). Mediation in experimental and nonexperimental studies: New procedures and recommendations. Psychological Methods, 7, 422-445.

Slovic, P. (1969). Differential effects of real versus hypothetical payoffs on choices among gambles. Journal of Experimental Psychology, 80, 434-437.

Sobel, M. E. (1982). Asymptotic confidence intervals for indirect effects in structural equation models. In S. Leinhart (Ed.), Sociological methodology 1982 (pp. 290-312. San Francisco: Jossey-Bass.

Soman, D. (2001). The mental accounting of sunk time costs: Why time is not like money. Journal of Behavioral Decision Making, 14, 169-185.

Soman, D. (2004). Framing, loss aversion, and mental accounting. In D. J. Koehler \& N. Harvey (Eds.), Blackwell handbook of judgment and decision making (pp. 379-398). Malden, MA: Blackwell.

Spencer, M. A., Swallow, S. K., \& Miller, J. M. (1998). Valuing water quality monitoring: A contingent valuation experiment involving hypothetical and real payments. Agricultural and Resource Economics Review, 28-42. 
Stanovich, K.E., \& West, R.F. (1999). Discrepancies between normative and descriptive models of decision making and the understanding/acceptance principle. Cognitive Psychology, $38,349-385$.

Stanovich, K. E., \& West, R. F. (2000). Individual differences in reasoning: Implications for the rationality debate? Behavioral and Brain Sciences, 23, 645-726.

Staw, B.M. (1976). Knee-deep in the big muddy: A study of escalating commitment to a chosen course of action. Organizational Behavior and Human Performance, 16, 27-44.

Staw, B.M. (1981). The escalation of commitment to a course of action. Academy of Management Review, 6(4), 577-587.

Staw, B.M., \& Hoang, H. (1995). Sunk costs in the NBA: Why draft order affects playing time and survival in professional basketball. Administrative Science Quarterly, 40(3), 474494.

Steinberg, L., Graham, S., O’Brien, L., Woolard, J., Cauffman, E., \& Banich, M. (2009). Age differences in future orientation and delay discounting. Child Development, 80(1), 28-44.

Steptoe, A., Pollard, T. M., \& Wardle, J. (1995). Development of a measure of the motives underlying the selection of food: The Food Choice Questionnaire. Appetite, 25, 267-284.

Strough, J., Karns, T. E., \& Schlosnagle, L. (2011a). Decision making heuristics and biases across the life span. Annals of the New York Academy of Sciences, 1235, 57-74. Strough, J., Mehta, C.M., McFall, J.P., \& Schuller, K.L. (2008). Are older adults less subject to the sunk-cost fallacy than younger adults? Psychological Science, 19(7), 650-652.

Strough, J., Schlosnagle, L., \& DiDonato, L. (2011b). Understanding decisions about sunk costs from older and younger adults' perspectives. Journal of Gerontology: Psychological Sciences. 
Tabachnick, B. G., \& Fidell, L. S. (2007). Using multivariate statistics (5th ed.). Boston: Pearson.

Tambour, M., \& Zethraeus, N. (1998). Nonparametric willingness-to-pay measures and confidence statements. Medical Decision Making, 18(3), 330-336.

Teas, K. R., \& Agarwal, S. (2000). The effects of extrinsic product cues on consumers' perceptions of quality, sacrifice, and value. Journal of the Academy of Marketing Science, 28, 278-290.

Tan, H., \& Yates, J.F. (1995). Sunk cost effects: The influences of instruction and future return estimates. Organizational Behavior and Human Decision Processes, 63(3), 311-319.

Thaler, R.H., \& Sunstein, C.R. (2008). Nudge: Improving decisions about health, wealth, and happiness. New Haven, Ct: Yale University Press.

Thaler, R.H., Tversky, A., Kahneman, D., \& Schwartz, A. (1997). The effects of myopia and loss aversion on risk taking: An experimental test. The Quarterly Journal of Economics, 112(2), 647-661.

Ting, H. (2011). The effects of goal distance and value in escalation of commitment. Current Psychology, 30, 93-104.

Tversky, A., \& Kahneman, D. (1991). Loss aversion in riskless choice: A reference-dependent model. The Quarterly Journal of Economics, 106(4), 1039-1061.

van Putten, M., Zeelenberg, M., \& van Dijk, E. (2010). Who throws good money after bad? Action vs. state orientation moderates the sunk cost fallacy. Judgment and Decision Making, 5(1), 33-36. 
Weller, J. A., Levin, I. P., \& Denburg, N. L. (2009). Trajectory of adaptive decision making for risky gains and losses from ages 5 to 85. Journal of Behavioral Decision Making, 22, 114.

Whelan, R., \& McHugh, L. A. (2009). Temporal discounting of hypothetical monetary rewards by adolescents, adults, and older adults. The Psychological Record, 59(2), 247-258.

Widaman, K. F. (2005). Missing data: What to do with or without them. In Monographs of the Society for Research in Child Development, 71 (3, Serial No. 285).

Wood, S., Busemeyer, J., Koling, A., Cox, C.R., \& Davis, H. (2005). Older adults as adaptive decision makers: Evidence from the Iowa gambling task. Psychology and Aging, 20(2), $220-225$.

Yaniv, I. (2004). Receiving other people's advice: influence and benefit. Organizational Behavior and Human Decision Processes, 93, 1-13.

Zikmund-Fisher, B. J., Smith, D. M., Ubel, P. A., \& Fagerlin, A. (2007). Validation of the Subjective Numeracy Scale: Effects of low numeracy on comprehension of risk communications and utility elicitations. Medical Decision Making, 27(5), 663-671. 
Table 1

Experimental Design of Calorie Estimation Task

\begin{tabular}{|c|c|c|c|c|c|c|c|c|}
\hline & \multicolumn{4}{|c|}{ Sequence 1} & \multicolumn{4}{|c|}{ Sequence 2} \\
\hline & \multicolumn{4}{|c|}{ Phase } & \multicolumn{4}{|c|}{ Phase } \\
\hline & 1 & 2 & 3 & 4 & 1 & 2 & 3 & 4 \\
\hline Advice Available? & no & yes & no & yes & no & yes & no & yes \\
\hline Price of Advice Cond. & & small & & large & & large & & small \\
\hline
\end{tabular}

*Note: Sequence was alternated for half of participants (i.e. the first half of participants were assigned to either Sequence 1, the second half of participants were assigned to Sequence 2). Phase was a within-subjects variable (i.e., all participants underwent all 4 Phases). 
Table 2

Demographic Characteristics Separated by Age Group

\begin{tabular}{|c|c|c|c|c|c|c|}
\hline & \multicolumn{3}{|c|}{ Younger Adults $(n=50)$} & \multicolumn{3}{|c|}{ Older Adults $(n=50)$} \\
\hline & $M$ & $S D$ & range & $M$ & $S D$ & range \\
\hline Age & 20.44 & 2.52 & $18-31$ & 69.74 & 6.85 & $60-85$ \\
\hline \multirow[t]{2}{*}{ Years of education } & 13.60 & 1.23 & $12-16$ & 15.91 & 2.77 & $12-20$ \\
\hline & \multicolumn{3}{|c|}{ Percentage } & \multicolumn{3}{|c|}{ Percentage } \\
\hline
\end{tabular}

Sex

Men

Women

Race

White/Caucasian

Black/African-American

Bi- or Multi-Racial

Native American

"prefer not to answer"

(Continued on next page)
$32 \%$

$68 \%$

$56 \%$

$44 \%$

$94 \%$

$96 \%$

$4 \%$

$0 \%$

$2 \%$

0\%

$0 \%$

$2 \%$

$0 \%$

$2 \%$ 
Table 2 (Continued from previous page)

$\frac{\text { Younger Adults }(n=50)}{\text { Percentage }}$

Older Adults $(n=50)$

Percentage

Ethnicity

$\begin{array}{lll}\text { Hispanic or Latino } & 0 \% & 0 \% \\ \text { Not Hispanic or Latino } & 100 \% & 88 \% \\ \text { "prefer not to answer" } & 0 \% & 12 \%\end{array}$

Employment Status

$\begin{array}{ccc}\text { Employed full time } & 12 \% & 16 \% \\ \text { Employed part time } & 34 \% & 6 \% \\ \text { Partially retired } & 0 \% & 10 \% \\ \text { Fully retired } & 0 \% & 58 \% \\ \text { Unemployed } & 40 \% & 4 \% \\ \text { "Other" } & 6 \% \\ & 14 \% & \\ & & \\ & & \\ & & \\ & & \end{array}$


Table 2 (Continued from previous page)

\begin{tabular}{c} 
Younger Adults $(n=50)$ \\
Percentage \\
\hline
\end{tabular}

Older Adults $(n=50)$

Percentage

Annual Income

Less than $\$ 10,000$

$\$ 10,000-19,000$

$\$ 20,000-29,000$

$\$ 30,000-39,000$

$\$ 40,000-49,000$

$\$ 50,000-59,000$

$\$ 60,000-69,000$

$\$ 70,000$ or more

"I don't know"
$64 \%$

$10 \%$

$2 \%$

$0 \%$

$0 \%$

$0 \%$

$0 \%$

$0 \%$

$24 \%$
$4 \%$

$10 \%$

$6 \%$

$22 \%$

$6 \%$

$16 \%$

$2 \%$

$28 \%$

$6 \%$ 
Table 2 (Continued from previous page)

Younger Adults $(n=50)$

Percentage

Class Rank (Only younger adults responded to this item)

$\begin{array}{rc}\text { Freshman } / 1^{\text {st }} \text { year } & 28 \% \\ \text { Sophomore } / 2^{\text {nd }} \text { year } & 16 \% \\ \text { Junior } / 3^{\text {rd }} \text { year } & 26 \% \\ \text { Senior } / 4^{\text {th }} \text { year } & 28 \% \\ \text { Graduate student } & 0 \% \\ \text { Other } & 2 \%\end{array}$

(Continued on next page) 
Table 2 (Continued from previous page)

Younger Adults $(n=50)$

Percentage

College funding (Only younger adults responded to this item)

Student pays for education and living expenses

Parents pay for education, student pays for living expenses

Parents pay for living expenses, student pays for education

Parents pay for education and living expenses

Other
$22 \%$

$22 \%$

$6 \%$

$22 \%$

$28 \%$

(Continued on next page) 
Table 2 (Continued from previous page)

Younger Adults $(n=50)$

Percentage

Parents' Income (Only younger adults responded to this item)

Less than $\$ 10,000$

$\$ 10,000-19,000$

$\$ 20,000-29,000$

$\$ 30,000-39,000$

$\$ 40,000-49,000$

$\$ 50,000-59,000$

$\$ 60,000-69,000$

$\$ 70,000$ or more

"I don't know"
$0 \%$

$6 \%$

$12 \%$

$4 \%$

$4 \%$

$4 \%$

$6 \%$

$28 \%$

$36 \%$

(Continued on next page) 
Table 2 (Continued from previous page)

Older Adults $(n=50)$

Percentage

Income prior to retirement (Only retired older adults responded)

Less than $\$ 10,000$

$\$ 10,000-19,000$

$\$ 20,000-29,000$

$\$ 30,000-39,000$

$\$ 40,000-49,000$

$\$ 50,000-59,000$

$\$ 60,000-69,000$

$\$ 70,000$ or more

"I don’t know"

No response
$2 \%$

$12 \%$

$0 \%$

$14 \%$

$16 \%$

$8 \%$

$0 \%$

$26 \%$

$4 \%$

$18 \%$ 
Table 3

Descriptive Statistics for Primary Variables

\begin{tabular}{|c|c|c|c|c|}
\hline Variable & $N$ & $M$ & $S D$ & range \\
\hline Qual. Adv. Score (small) & 50 & 4.51 & .79 & $2.50-6$ \\
\hline Qual. Adv. Score (large) & 34 & 4.45 & .81 & $3-6$ \\
\hline Est. Diff. Score (small) & 100 & 791.22 & 535.94 & $81-2716$ \\
\hline Est. Diff. Score (large) & 100 & 628.27 & 385.53 & $100-2107$ \\
\hline Weight of Adv. (small) & 50 & 8.87 & 3.61 & $1.50-15$ \\
\hline Weight of Adv. (large) & 34 & 8.17 & 3.35 & $2.40-15$ \\
\hline Cal. Snk. Cst. Fllcy. & 32 & 6.28 & 3.52 & $0-13$ \\
\hline Advice Adherence Score (sml) & 50 & 875.04 & 630.24 & $-47-2660$ \\
\hline Advice Adherence Score (lrg) & 34 & 672.13 & 500.01 & $-160-2067$ \\
\hline Tot. Adv. Adhnc. SCF Score & 32 & 5.94 & 3.27 & $1-13$ \\
\hline Pre-Adv. Confidence Int. & 100 & 383.81 & 55.78 & $237-483.33$ \\
\hline Pre-Adv. Accuracy & 100 & 6.87 & 4.15 & $0-18$ \\
\hline Final Winnings Score & 100 & 5.19 & 2.55 & $0-10$ \\
\hline Delay Disc. Loss Av. & 46 & -.12 & .21 & $-.65-.35$ \\
\hline R. AUC Gains Score & 46 & .58 & .20 & $.09-.99$ \\
\hline R. AUC Losses Score & 46 & .71 & .27 & $.27-.99$ \\
\hline Trade. Loss Av. & 100 & 383.12 & 140.74 & $0-495$ \\
\hline Snk. Cst. Fllcy. Vignette & 100 & .84 & .65 & $0-2$ \\
\hline Snk. Cst. Fllcy. Overinvest. & 100 & 1.90 & 1.64 & $0-6$ \\
\hline Norm. Correct Score & 100 & .67 & .62 & $0-2$ \\
\hline Sub. Num. Scale & 100 & 3.89 & .95 & $1-6$ \\
\hline
\end{tabular}


Table 3 (Continued from previous page)

\begin{tabular}{|c|c|c|c|c|}
\hline Variable & $N$ & $M$ & $S D$ & range \\
\hline Food Choice Qst. Score & 100 & 2.75 & .80 & $1-4$ \\
\hline Health Habits Qst. Score & 100 & 2.17 & .35 & $1.32-2.86$ \\
\hline Spndthft. Ttwd. Scale & 100 & 14.96 & 3.87 & $4-25$ \\
\hline Futr. Time Persp. Score & 100 & 4.66 & 1.31 & $1.40-7$ \\
\hline Difficulty Paying Bills Score & 100 & .85 & .97 & $0-4$ \\
\hline
\end{tabular}

Note: Qual. Adv. Scores (small, large)=Quality of Advice Scores for the small and large price of advice conditions, respectively; Est. Diff. Score (small, large)=Estimate Difference Score for the small and large price of advice conditions, respectively; Weight of Adv. (small, large)=Small, Large Price of Advice Condition Weight of Advice Scores, respectively; Cal. Snk. Cst Fllcy. = Calorie Estimate Sunk-Cost Fallacy Score; Advice Adherence Score (sml, lrg)= Small, Large Price of Advice Condition Advice Adherence Scores, respectively; Tot. Adv. Adhnc. SCF Score=Total Advice Adherence Sunk-Cost Fallacy Score; Pre-Adv. Confidence Int.=Mean PreAdvice Confidence Interval Score; Pre-Adv. Accuracy.=Pre-Advice Calorie Estimate Accuracy Score; Delay Disc. Loss Av.=Delay Discounting Loss Aversion Score; R. AUC Gains and Losses Scores=Reversed Area Under the Curve Gains, and Losses Scores, respectively; Trade. Loss Av. $=$ Tradeoff Loss Aversion Task Score; Snk Cst. Fllcy. Vignette=Sunk-Cost Fallacy Vignette Score; Snk. Cst. Fllcy. Overinvest.=Sunk-Cost Fallacy Vignette Overinvestment Score; Norm. Correct Score=Normatively Correct Score; Sub. Num. Scale=Subjective Numeracy Scale Score; Food Choice Qst. Score=Food Choice Questionnaire; Health Habits Qst. Score=Health Habits Questionnaire; Spndthft. Ttwd Scale=Spendthrift-Tightwad Scale Score; Futr. Time Persp. Score=Future Time Perspective Score. 
Table 4

Descriptive Statistics for Primary Variables, Separated by Age Group

\begin{tabular}{|c|c|c|c|c|c|c|}
\hline \multirow[b]{2}{*}{ Variable } & \multicolumn{3}{|c|}{ Younger Adults } & \multicolumn{3}{|c|}{ Older Adults } \\
\hline & $M$ & $S D$ & range & $M$ & $S D$ & range \\
\hline Qual. Adv. Score (small) & $4.17^{14}$ & .75 & $3-5$ & $4.59^{36}$ & .78 & $2.50-6$ \\
\hline Qual. Adv. Score (large) & $4.00^{8}$ & .82 & $3-5$ & $4.52^{26}$ & .80 & $3-6$ \\
\hline Est. Diff. Score (small) & 742.73 & 589.88 & $81-2716$ & 839.71 & 477.04 & $83-2040$ \\
\hline Est. Diff. Score (large) & 574.84 & 299.79 & $100-1322$ & 681.71 & 452.32 & $120-2107$ \\
\hline Weight of Adv. (small) & $9.55^{14}$ & 4.22 & $1.50-15$ & $8.60^{36}$ & 3.37 & $3.20-15$ \\
\hline Weight of Adv. (large) & $7.44^{8}$ & 3.79 & $2.40-13.05$ & $8.40^{26}$ & 3.25 & $2.85-15$ \\
\hline Cal. Snk. Cst. Fllcy. & $5.67^{6}$ & 3.67 & $1-9$ & $6.42^{26}$ & 3.54 & $0-13$ \\
\hline Adv. Adherence Score (sml) & $881.82^{14}$ & 945.19 & $-47-2660$ & $872.40^{36}$ & 473.53 & $254-2040$ \\
\hline Adv. Adherence Score (lrg) & $310.12^{8}$ & 428.53 & $-160-860$ & $783.52^{26}$ & 473.02 & $286-2067$ \\
\hline Tot. Adv. Adhnc. SCF Score & $5.00^{6}$ & 3.22 & $1-10$ & $6.15^{26}$ & 3.31 & $1-13$ \\
\hline Pre-Adv. Confidence Int. & 356.33 & 56.48 & $237-471$ & 411.29 & 39.43 & $344-483$ \\
\hline Pre-Adv. Accuracy & 6.96 & 4.35 & $0-17$ & 6.78 & 3.99 & $1-18$ \\
\hline Final Winnings Score & 5.02 & 2.54 & $.50-10$ & 5.56 & 2.38 & $1.50-10$ \\
\hline Delay Disc. Loss Av. & $-.20^{23}$ & .22 & $-.65-.35$ & $-.04^{23}$ & .16 & $-.39-.27$ \\
\hline R. AUC Gains Score & $.58^{23}$ & .21 & $.21-.99$ & $.57^{23}$ & .20 & $.09-.97$ \\
\hline R. AUC Losses Score & $.79^{23}$ & .17 & $.29-.99$ & $.63^{23}$ & .23 & $.27-.99$ \\
\hline Trade. Loss Av. & 444.89 & 50.49 & $346-492$ & 321.33 & 172.29 & $0-495$ \\
\hline Snk. Cst. Fllcy. Vignette & 1.02 & .59 & $0-2$ & .66 & .66 & $1-2$ \\
\hline Snk. Cst. Fllcy. Overinvest & 2.66 & 1.64 & $0-6$ & 1.14 & 1.24 & $0-4$ \\
\hline
\end{tabular}


Table 4 (Continued from previous page)

Descriptive Statistics for Primary Variables, Separated by Age Group

\begin{tabular}{|c|c|c|c|c|c|c|}
\hline \multirow[b]{2}{*}{ Variable } & \multicolumn{3}{|c|}{ Younger Adults } & \multicolumn{3}{|c|}{ Older Adults } \\
\hline & $M$ & $S D$ & range & $M$ & $S D$ & range \\
\hline Norm. Correct Score & .54 & .54 & $0-2$ & .80 & 67 & $0-2$ \\
\hline Sub. Num. Scale & 3.79 & .96 & $1-5.88$ & 3.98 & .94 & $1.63-6$ \\
\hline Food Choice Qst. Score & 2.63 & .85 & $1-4$ & 2.88 & .75 & $1-4$ \\
\hline Health Habits Qst. Score & 2.08 & .34 & $1.32-2.86$ & 2.26 & .34 & $1.36-2.86$ \\
\hline Spndthft. Ttwd. Scale & 15.48 & 4.47 & $4-24$ & 14.44 & 3.11 & $6-25$ \\
\hline Futr. Time Persp. Score & 5.61 & .86 & $3.30-7$ & 3.71 & .95 & $1.40-5.40$ \\
\hline Difficulty Paying Bills Score & 1.30 & .95 & $0-4$ & .40 & .75 & $0-3$ \\
\hline
\end{tabular}

Note: $n=50$ for each age group, unless indicated otherwise with numeric superscript next to mean value (in which case, the value of the superscript is $n$ ).

Qual. Adv. Scores (small, large)=Quality of Advice Scores for the small and large price of advice conditions, respectively; Est. Diff. Score (small, large)=Estimate Difference Score for the small and large price of advice conditions, respectively; Weight of Adv. (small, large)=Small, Large Price of Advice Condition Weight of Advice Scores, respectively; Cal. Snk. Cst Fllcy. = Calorie Estimate Sunk-Cost Fallacy Score; Advice Adherence Score (sml, lrg)= Small, Large Price of Advice Condition Advice Adherence Scores, respectively; Tot. Adv. Adhnc. SCF Score $=$ Total Advice Adherence Sunk-Cost Fallacy Score; Pre-Adv. Confidence Int.=Mean PreAdvice Confidence Interval Score; Pre-Adv. Accuracy.=Pre-Advice Calorie Estimate Accuracy Score; Delay Disc. Loss Av.=Delay Discounting Loss Aversion Score; R. AUC Gains and Losses Scores=Reversed Area Under the Curve Gains, and Losses Scores, respectively; Trade. Loss Av. $=$ Tradeoff Loss Aversion Task Score; Snk Cst. Fllcy. Vignette=Sunk-Cost Fallacy Vignette Score; Snk. Cst. Fllcy. Overinvest.=Sunk-Cost Fallacy Vignette Overinvestment Score; Norm. Correct Score=Normatively Correct Score; Sub. Num. Scale=Subjective Numeracy Scale Score; Food Choice Qst. Score=Food Choice Questionnaire; Health Habits Qst. Score=Health Habits Questionnaire; Spndthft. Ttwd Scale=Spendthrift-Tightwad Scale Score; Futr. Time Persp. Score=Future Time Perspective Score. 
Table 5

Pearson Correlations for Primary Variables (Entire Sample; Younger and Older Adults Combined)

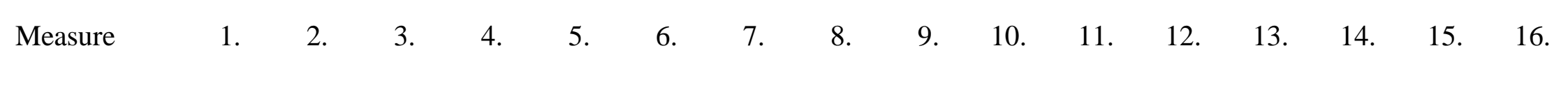

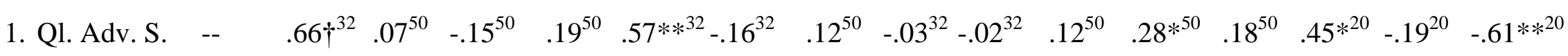

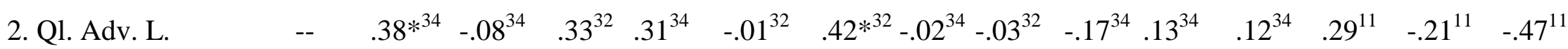

3. Est. Dff. S. $\quad--\quad .46 \dagger \quad .28 *^{50} \quad .19^{34}-.06^{32} \quad .97 \dagger^{50} \quad .23^{34}-.40 * 32-.41 \dagger-.32 * * \quad-.21 *^{34} \quad .45 * *^{46} .43 * * 46-.05^{46}$

4. Est. Dff. L.

$\begin{array}{llllllllllll}-- & .07^{50} & .20^{34} & .09^{32} & .37 * * 50 & .97 \dagger^{34} & .62 \dagger^{32}-.30 * * & -.39 \dagger & -.17 & .14^{46} & .05^{46} & -.11^{46}\end{array}$

5. WOA S.

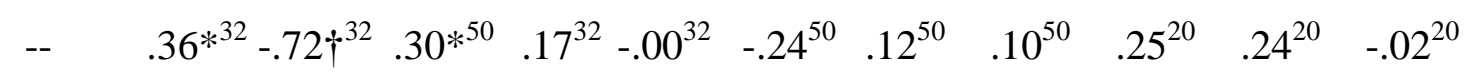

6. WOA L.

7. Cal. SCF.

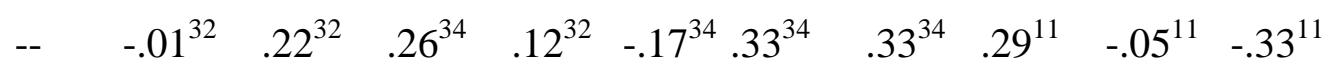

8. AS. S.

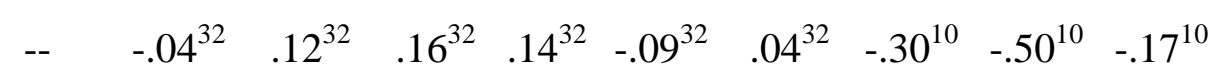

8. AAS. S.

9. AAS. L.

$--\quad .29^{32}-.38 *^{32}-.53 \uparrow^{50}-.30^{* 50}-.26^{50} .59 * *^{20} .58^{* * 20}-.05^{20}$

10. AAS SCF

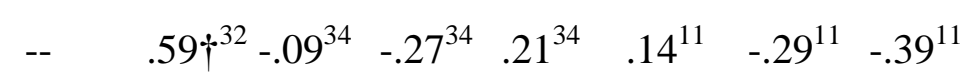

(Continued on next page) 
Table 5 (Continued from previous page)

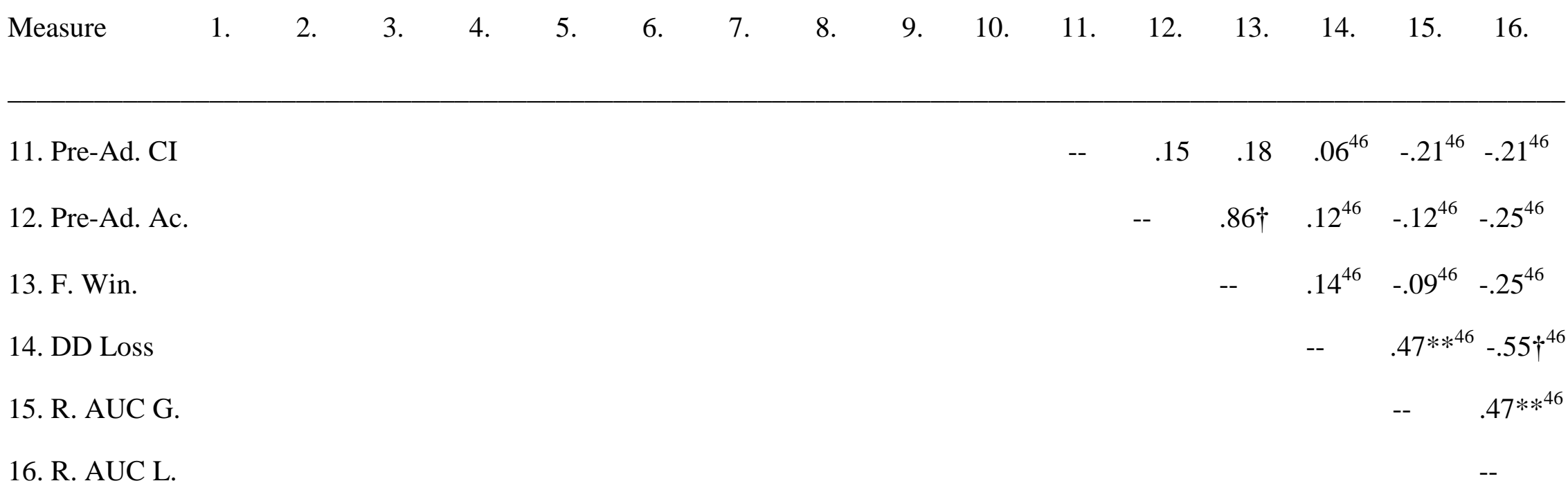


Table 5 (Continued from previous page)

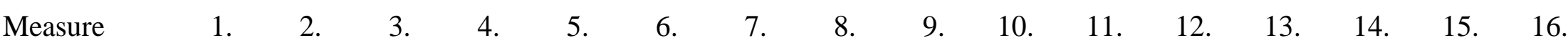

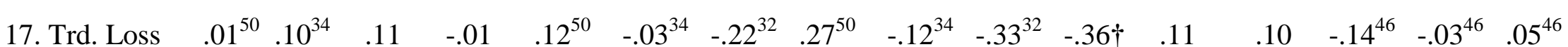

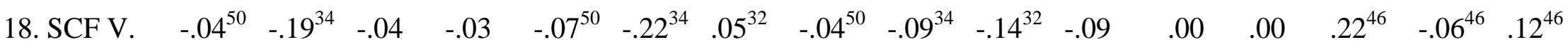

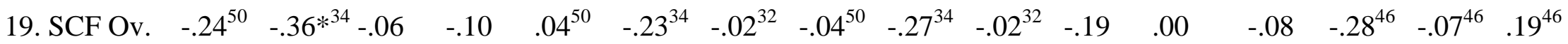

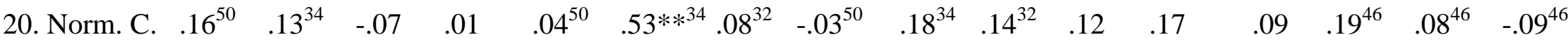

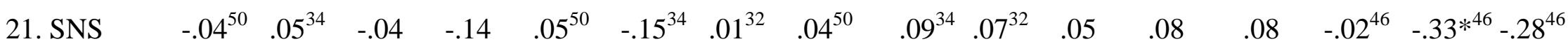

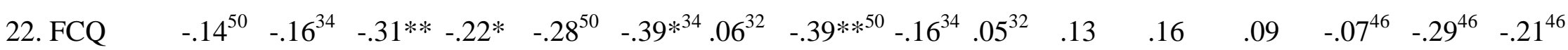

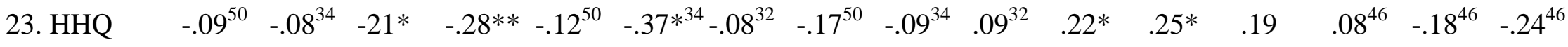

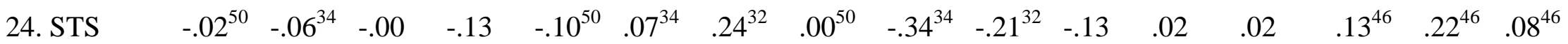

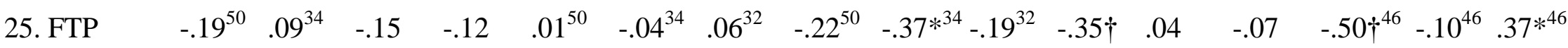

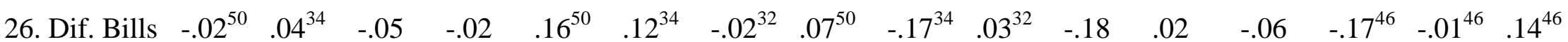


Table 5 (Continued from previous page)

$\begin{array}{llllllllll}\text { Measure } & 17 . & 18 . & 19 . & 20 . & 21 . & 22 . & 23 . & 24 . & 25 .\end{array}$

\begin{tabular}{|c|c|c|c|c|c|c|c|c|c|c|}
\hline 17. Trd. Loss & -- & .13 & .18 & -.11 & .09 & -.19 & $-.27 * *$ & -.05 & $.38 \uparrow$ & .09 \\
\hline 18. SCF V. & & -- & $.75 \dagger$ & $-.59 \dagger$ & .08 & -.02 & .04 & -.01 & $.23^{*}$ & .07 \\
\hline 19. SCF Ov. & & & -- & $-.56 \dagger$ & .14 & .01 & -.01 & -.09 & $.32 * *$ & $.26 * *$ \\
\hline 20. Norm. C. & & & & -- & -.04 & -.06 & -.05 & .03 & -.08 & -.05 \\
\hline 21. SNS & & & & & -- & $.22 *$ & .18 & $-.02^{50}$ & -.13 & -.16 \\
\hline 22. FCQ & & & & & & -- & $.63 \dagger$ & -.18 & -.12 & -.17 \\
\hline 23. HHQ & & & & & & & -- & $-.25^{*}$ & -.19 & -.16 \\
\hline 24. STS & & & & & & & & -- & .09 & .12 \\
\hline 25. FTP & & & & & & & & & -- & $.37 \dagger$ \\
\hline 26. Dif. Bills & & & & & & & & & & -- \\
\hline
\end{tabular}

(Continued on next page) 
Table 5 (Continued from previous page)

Note. $N=100$, except correlations with numerical superscripts, which indicate $N$. Q1. Adv. (S., L.)=Quality of Advice Scores for the small and large price of advice conditions, respectively; Est. Dff. (S., L.)=Estimate Difference Score for the small and large price of advice conditions, respectively; WOA (S., L.)=Small, Large Price of Advice Condition Weight of Advice Scores, respectively; Cal. SCF = Calorie Estimate Sunk-Cost Fallacy Score; AAS (S., L.)= Small, Large Price of Advice Condition Advice Adherence Scores, respectively; AAS SCF=Total Advice Adherence Sunk-Cost Fallacy Score; Pre-Ad. CI=Mean Pre-Advice Confidence Interval Score; Pre-Ad. Ac. $=$ Pre-Advice Calorie Estimate Accuracy Score; F. Win.=Final Winnings Score; DD Loss=Delay Discounting Loss Aversion Score; R. AUC (G., L.)=Reversed Area Under the Curve Gains, and Losses Scores, respectively; Trd. Loss=Tradeoff Loss Aversion Task Score; SCF V.=Sunk-Cost Fallacy Vignette Score; SCF OV.=Sunk-Cost Fallacy Vignette Overinvestment Score; Norm. C.=Normatively Correct Score; SNS=Subjective Numeracy Scale Score; FCQ=Food Choice Questionnaire; HHQ=Health Habits Questionnaire; STS=Spendthrift-Tightwad Scale Score; FTP=Future Time Perspective Score; Dif. Bills=Difficulty Paying Bills Score. $* p<.05, * * p<.01, \dagger p<.001$ (2-tailed). 
Table 6

Pearson Correlations for Primary Variables by Age Group (Correlations Above the Diagonal are for Younger Adults, Correlations Below the Diagonal are for Older Adults)

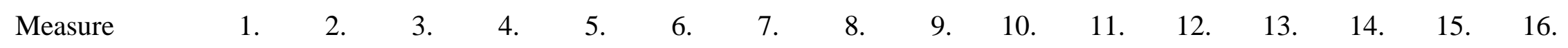

1. Q1. Adv. S. $\quad--\quad \begin{array}{lllllllllllllllll} & .62^{6} & .43^{14} & -.01^{14} & .38^{14} & .27^{6} & -.79^{6} & .38^{14} & -.15^{6} & -.46^{6} & -.48^{14} & -.23^{14} & -.37^{14} & .47^{7} & .22^{7} & -.63^{7}\end{array}$

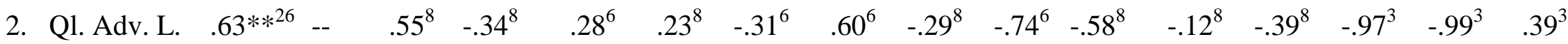

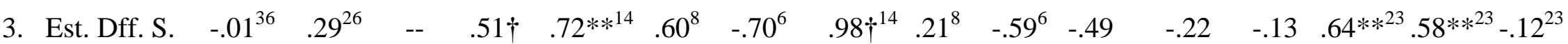

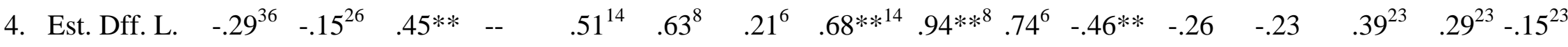

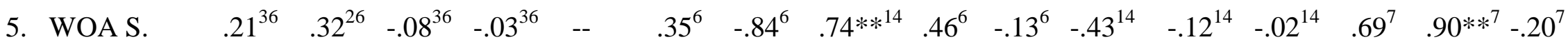

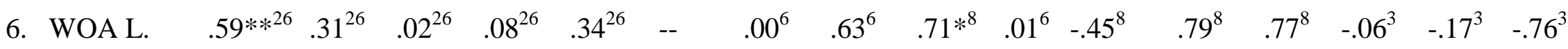

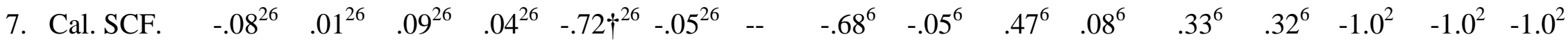

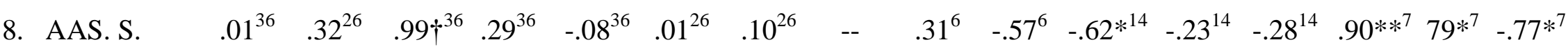

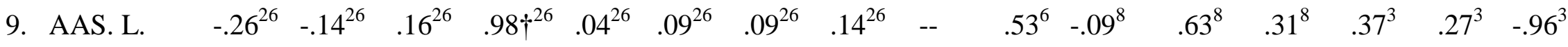

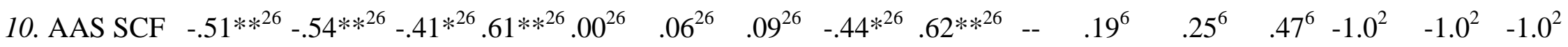

(Continued on next page) 
Table 6 (Continued from previous page)

\begin{tabular}{|c|c|c|c|c|c|c|c|c|c|c|c|c|c|c|c|c|}
\hline Measure & 1. & 2. & 3. & 4. & 5. & 6. & 7. & 8. & 9. & 10. & 11. & 12. & 13. & 14. & 15. & 16. \\
\hline 11. Pre-Ad. CI & $.06^{36}$ & $-.25^{26}$ & $-.59 \dagger$ & $-.46 * *$ & $-.05^{36}$ & $-.14^{26}$ & $.13^{26}$ & $-.66 \dagger^{36}$ & $-.39 * 26$ & $.06^{26}$ & -- & .17 & .16 & $-.14^{23}$ & $-.23^{23}$ & $-.09^{23}$ \\
\hline 12. Pre-Ad. Ac. & $.50 * * 36$ & $6.33^{26}$ & $-.47 * *$ & $-.50 \dagger$ & $.24^{36}$ & $.26^{26}$ & $-.19^{26}$ & $-.39 * 36$ & $-.41 * 26$ & $-.26^{26}$ & .22 & -- & $.89 \dagger$ & $.11^{23}$ & $-.24^{23}$ & $-.45^{* 23}$ \\
\hline 13. F. Win. & $.37 *^{36}$ & $.26 * * 26$ & $-.35^{*}$ & -.17 & $.16^{36}$ & $.15^{26}$ & $-.04^{26}$ & $-.27^{36}$ & $.04^{26}$ & $-.04^{26}$ & .13 & $.83 \dagger$ & -- & $.14^{23}$ & $-.10^{23}$ & $-.31^{23}$ \\
\hline 14. DD Loss & $.56^{* 13}$ & $.05^{8}$ & $.13^{23}$ & $-.12^{23}$ & $-.08^{13}$ & $.05^{8}$ & $-.33^{8}$ & $-.01^{13}$ & $-.51^{8}$ & $-.56^{8}$ & $-.19^{23}$ & $.19^{23}$ & $.11^{23}$ & -- & $.68 \dagger^{23}$ & $-.46^{23}$ \\
\hline 15. R. AUC G. & $-.20^{13}$ & $-.15^{8}$ & $.25^{23}$ & $-.08^{23}$ & $-.09^{13}$ & $-.05^{8}$ & $-.41^{8}$ & $.52^{13}$ & $-.44^{8}$ & $-.25^{8}$ & $-.22^{23}$ & $.00^{23}$ & $-.08^{23}$ & $.28^{23}$ & -- & $.33^{23}$ \\
\hline 16. R. AUC L. & $-.60 * 13$ & $-.21^{8}$ & $.06^{23}$ & $-.03^{23}$ & $-.01^{13}$ & $-.08^{8}$ & $-.17^{8}$ & $.49^{13}$ & $-.07^{8}$ & $.19^{8}$ & $.01^{23}$ & $-.16^{23}$ & $-.19^{23}$ & $-.54 * * 23$ & $.64 * * 2$ & 23 \\
\hline
\end{tabular}


Table 6 (Continued from previous page)

Correlations below are for younger adults only:

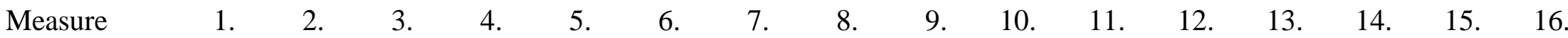

$\begin{array}{lllllllllllllllllll}\text { 17. Trd. Loss } & .44^{14} & -.03^{8} & .03 & .04 & .43^{14} & .03^{8} & -.36^{6} & .30^{14} & -.13^{8} & .33^{6} & -.09 & -.13 & -.18 & -.03^{23} & .14^{23} & .22^{23} \\ \text { 18. SCF V. } & .01^{14} & -.56^{8} & -.17 & -.08 & -.11^{14} & -.30^{8} & -.25^{6} & -.24^{14} & -.14^{8} & .60^{6} & .12 & .14 & .13 & -.06^{23} & .09^{23} & .19^{23} \\ \text { 19. SCF Ov. } & .12^{14} & .08^{8} & -.05 & -.11 & .03^{14} & -.36^{8} & .18^{6} & .04^{14} & -.23^{8} & .31^{6} & .06 & .12 & .09 & -.15^{23} & -.07^{23} & .11^{23} \\ \text { 20. Norm. C. } & .03^{14} & .00^{8} & -.06 & .12 & -.11^{14} & .18^{8} & .44^{6} & -.14^{14} & .44^{8} & .15^{6} & .02 & .03 & -.00 & -.11^{23} & -.00^{23} & -.15^{23} \\ \text { 21. SNS } & .15^{14} & .32^{8} & -.11 & -.35^{*} & .14^{14} & .59^{8} & .09^{6} & -.03^{14} & -.15^{8} & .12^{6} & .01 & .16 & .14 & -.22^{23} & -.25^{23} & -.01^{23} \\ \text { 22. FCQ } & -.28^{14} & -.16^{8} & -.48 \dagger & -.41^{* *} & -.59^{14} & .42^{8} & .57^{6} & -69^{* * 14} & -.49^{8} & -.02^{6} & .10 & .35^{*} & .22 & -.27^{23} & -.38^{23} & -.10^{23} \\ \text { 23. HHQ } & -.57^{14} & -.13^{8} & -.24 & -.33^{*} & -.24^{14} & -.53^{8} & .43^{6} & -.15^{14} & -.03^{8} & -.09^{6} & .13 & .29^{*} & .19 & -.14^{23} & -.29^{23} & -.16^{23} \\ \text { 24. STS } & -.09^{14} & -.03^{8} & .09 & .16 & .09^{14} & .69^{8} & .13^{6} & .06^{14} & .32^{8} & -.13^{6} & -.13 & -.03 & .08 & .20^{23} & .43^{23} & .25^{23} \\ \text { 25. FTP } & -.22^{14} & -.08^{8} & -.17 & -.10 & -.27^{14} & -.78^{*} & .28^{6} & -.70^{* * 14} & -.86^{* * 8} & .22^{6} & -.02 & -.08 & -.09 & -.50^{* 23} & -.38^{23} & .19^{23} \\ \text { 26. Dif. Bills } & .59^{14} & .70^{8} & .12 & .09 & .42^{14} & -.04^{8} & -.63^{6} & .43^{14} & -.28^{8} & -.86^{* 6} & .06 & -.11 & -.12 & .08^{23} & -.04^{23} & -.14^{23}\end{array}$


Table 6 (Continued from previous page)

Correlations below are for older adults only:

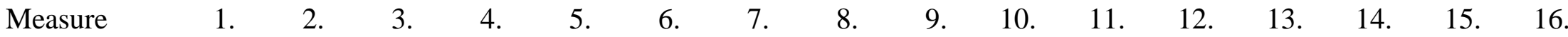

$\begin{array}{llllllllllllllllll}\text { 17. Trd. Loss } & .17^{36} & .24^{26} & .27 & .06 & .06^{36} & .00^{26} & -.20^{26} & .41^{36} & .02^{26} & -.36^{26} & -.28^{*} & .21 & .31^{*} & -.01^{23} & -.11^{23} & -.15^{23} \\ \text { 18. SCF V. } & .07^{36} & -.06^{26} & .16 & .05 & -.11^{36} & -.17^{26} & .14^{26} & .07^{36} & .02^{26} & -.21^{26} & -.05 & -.14 & -.06 & -.12^{23} & .24^{23} & -.17^{23} \\ \text { 19. SCF Ov. } & -.13^{36} & -. .36^{26} & .03 & .01 & -.07^{36} & -.35^{26} & .01^{26} & -.15^{36} & -.04^{26} & .04^{26} & .03 & -.19 & -.23 & -.05^{23} & -.12^{23} & -.10^{23} \\ \text { 20. Norm. C. } & .03^{36} & .10^{26} & -.12 & -.10 & .16^{36} & .50^{* * 26} & .00^{26} & .02^{36} & .04^{26} & .09^{26} & .02 & .30^{*} & .15 & .09^{23} & .16^{23} & .08^{23} \\ \text { 21. SNS } & -.16^{36} & -.08^{26} & .02 & -.04 & .03^{36} & -.43^{26} & -.03^{26} & .09^{36} & .08^{26} & .04^{26} & -.02 & .00 & -.01 & -.07^{23} & -.43^{23} & -.31^{23} \\ \text { 22. FCQ } & -.18^{36} & -.14^{26} & -.12 & -.13 & -.08^{36} & -.33^{26} & -.06^{26} & -.14^{36} & -.03^{26} & .08^{26} & .00 & -.05 & -.08 & -.06^{23} & -.19^{23} & -.14^{23} \\ \text { 23. HHQ } & -.08^{36} & -.10^{26} & -.26 & -.34^{*} & -.03^{36} & -.35^{26} & -.22^{26} & -.21^{36} & -.16^{26} & -12^{26} & .09 & .24 & .14 & .10^{23} & -.07^{23} & -.13^{23} \\ \text { 24. STS } & .04^{36} & -.05^{26} & -.13 & -.39 * * & -.24^{36} & -.14^{26} & .27^{26} & -.06^{36} & -.52^{*} * 26 & -.23^{26} & .05 & .09 & -.03 & .19^{23} & -.15^{23} & -.25^{23} \\ \text { 25. FTP } & .20^{36} & .4 *^{26} & -.08 & .01 & -.05^{36} & .25^{26} & .14^{26} & -.12^{36} & -.02^{26} & -.18^{26} & .04 & .15 & .12 & -.16^{23} & .01^{23} & .12^{23} \\ \text { 26. Dif. Bills } & .09^{36} & -.05^{26} & -.23 & .02 & -.11^{36} & .29^{26} & .27^{26} & -.34^{36} & .05^{26} & .47 * 26 & .08 & .19 & .13 & .15^{23} & -.02^{23} & -.13^{23}\end{array}$


Table 6 (Continued from previous page)

Correlations above diagonal are for younger adults, correlations below diagonal are for older adults

$\begin{array}{lllllllll}\text { Measure } & 17 . & 18 . & 19 . & 20 . & 21 . & 22 . & 23 . & 24 .\end{array}$

\begin{tabular}{lccccccccccc}
\hline 17. Trd. Loss & -- & .06 & $.28^{*}$ & -.08 & .12 & -.08 & -.25 & -.24 & .10 & .00 \\
18. SCF V. & -.00 & -- & $.73 \dagger$ & $-.42^{* *}$ & .21 & .04 & .09 & -.13 & .07 & .06 \\
19. SCF Ov. & -.16 & $.76 \dagger$ & -- & $-.46^{* *}$ & $.29 *$ & .06 & .08 & -.24 & -.04 & .18 \\
20. Norm. C. & .00 & $-.67 \dagger$ & $-.65 \dagger$ & -- & -.06 & -.01 & -.08 & .16 & .15 & -.12 \\
21. SNS & .19 & .01 & .10 & -.05 & -- & .27 & .17 & -.15 & -.09 & -.10 \\
22. FCQ & -.19 & -.00 & .14 & -.17 & .13 & -- & $.66 \dagger$ & -.27 & .08 & -.18 \\
23. HHQ & -.19 & .15 & .23 & -.15 & .16 & $.57 \dagger$ & -- & $-.43 * *$ & -.06 & -.04 \\
24. STS & -.13 & .03 & -.04 & -.04 & .21 & .00 & .06 & -- & .06 & .05 \\
25. FTP & .10 & .01 & -.02 & .08 & -.06 & -.10 & .06 & -.12 & -- & .03 \\
26. Dif. Bills & -.25 & -.21 & -.15 & .24 & -.17 & -.03 & -.05 & .06 & .06 & -- \\
\end{tabular}

(Continued on next page) 
Table 6 (Continued from previous page)

Note. $N=50$, except correlations with numerical superscripts, which indicate $N$. Q1. Adv. (S., L.)=Quality of Advice Scores for the small and large price of advice conditions, respectively; Est. Dff. (S., L.)=Estimate Difference Score for the small and large price of advice conditions, respectively; WOA (S., L.)=Small, Large Price of Advice Condition Weight of Advice Scores, respectively; Cal. SCF = Calorie Estimate Sunk-Cost Fallacy Score; AAS (S., L.)= Small, Large Price of Advice Condition Advice Adherence Scores, respectively; AAS SCF=Total Advice Adherence Sunk-Cost Fallacy Score; Pre-Ad. CI=Mean Pre-Advice Confidence Interval Score; Pre-Ad. Ac. $=$ Pre-Advice Calorie Estimate Accuracy Score; F. Win.=Final Winnings Score; DD Loss=Delay Discounting Loss Aversion Score; R. AUC (G., L.)=Reversed Area Under the Curve Gains, and Losses Scores, respectively; Trd. Loss=Tradeoff Loss Aversion Task Score; SCF V.=Sunk-Cost Fallacy Vignette Score; SCF OV.=Sunk-Cost Fallacy Vignette Overinvestment Score; Norm. C.=Normatively Correct Score; SNS=Subjective Numeracy Scale Score; FCQ=Food Choice Questionnaire; HHQ=Health Habits Questionnaire; STS=Spendthrift-Tightwad Scale Score; FTP=Future Time Perspective Score; Dif. Bills=Difficulty Paying Bills Score. $* p<.05, * * p<.01, \dagger p<.001$ (2-tailed). 
Table 7

Pearson Correlations: Weight Control Sub-Scale of the Food Choice Questionnaire Score, Health Habits Questionnaire Score, Mean Pre-Advice Confidence Interval Score, and PreAdvice Calorie Estimate Accuracy Score; for the Entire Sample (Younger and Older Adults Combined)

Measure

1.

2.

3.

4.

1. FCQ

$-$

$.63 \dagger$

.13

.17

2. HHQ

$--$

$.23 *$

$.25 * *$

3. Pre-Adv. CI

$\begin{array}{ll}-- & .15\end{array}$

4. Pre-Adv. Acc.

Note. $N=100$. FCQ $=$ Weight Control Sub-Scale of the Food Choice Questionnaire Score, HHQ = Health Habits Questionnaire Score, Pre-Adv. CI = Mean Pre-Advice Confidence Interval Score Pre-Adv. Acc. $=$ Pre-Advice Calorie Estimate Accuracy Score. $*_{p}<.05, * * p<.01, \uparrow p<$ .001 (2-tailed). 
Table 8

Pearson Correlations: Weight Control Sub-Scale of the Food Choice Questionnaire Score, Health Habits Questionnaire Score, Mean Pre-Advice Confidence Interval Score, and PreAdvice Calorie Estimate Accuracy Score for Younger and for Older Adults

Measure

1.

2.

3.

4.

\section{1. $\mathrm{FCQ}$}

$-$

$.66 \dagger$

.10

$.35^{*}$

2. HHQ

$.57 \dagger$

$--$

.13

$.29 *$

3. Pre-Adv. CI

.00

.09

$-$

.17

4. Pre-Adv. Acc.

$-.05$

.24

.22

$-$

Note. Correlations above the diagonal are for younger adults $(n=50)$. Correlations below the diagonal are for older adults $(n=50)$. FCQ $=$ Weight Control Sub-Scale of the Food Choice Questionnaire Score, HHQ = Health Habits Questionnaire Score, Pre-Adv. CI = Mean PreAdvice Confidence Interval Score, Pre-Adv. Acc. = Pre-Advice Calorie Estimate Accuracy Score. $* p<.05, * * p<.01, \dagger p<.001$ (2-tailed). 
Table 9

Pearson Correlations: the Subjective Numeracy Scale, Reversed AUC Gains Scores, Reversed AUC Losses Scores and FTP Scores for the Entire Sample (Younger and Older Adults Combined)

Measure

1.

2.

3.

4.

1. SNS

$--.33 *$

$-.28$

$-.13^{100}$

2. R. AUC Gains

$--\quad .47 * *$

$-.10$

3. R. AUC Losses

$\begin{array}{ll}-- & 37 *\end{array}$

4. FTP

Note. $n=46$ unless indicated otherwise in superscript. SNS = Subjective Numeracy Scale; R. AUC Gains = Reversed Area Under the Curve Gains Score, R. AUC Losses = Reversed Area Under the Curve Losses Score; FTP = Future Time Perspective Score. ${ }^{*} p<.05,{ }^{*} p<.01, \dagger p<$ .001 (two-tailed). 
Table 10

Pearson Correlations: the Subjective Numeracy Scale, Reversed AUC Gains Scores, Reversed AUC Losses Scores, and FTP Scores for Younger and for Older Adults

Measure

1.

2.

3.

4.

1. $\mathrm{SNS}$

$\begin{array}{ll}-- & -.25\end{array}$

$-.01$

$-.09^{50}$

2. R. AUC Gains

$-.44 *$

$-$

.33

$-.38$

3. R. AUC Losses

$-.32$

$.64 \dagger$

$--$

.19

4. FTP

$-.06^{50}$

.01

.12

--

Note. Correlations above the diagonal are for younger adults $(n=23$ unless indicated otherwise in superscript). Correlations below the diagonal are for older adults ( $n=23$ unless indicated otherwise in superscript). SNS = Subjective Numeracy Scale; R. AUC Gains = Reversed Area Under the Curve Gains Score, R. AUC Losses = Reversed Area Under the Curve Losses Score; FTP $=$ Future Time Perspective Score.. $* p<.05, * * p<.01, \uparrow p<.001$. 
Table 11

Delay Discounting Loss Aversion Score as a Mediator of the Relation Between Age Group and Sunk-Cost Fallacy Vignette Score

\begin{tabular}{llrrr} 
Requirement no. & Variable & $B$ & $S E B$ & $\beta$ \\
\hline 1 & Age Group-SCF V. & -.36 & .12 & $-.28^{* *}$ \\
$2 \mathrm{a} \quad$ & Age Group-DD Loss & .16 & .06 & $.38^{* *}$ \\
$2 \mathrm{~b}$ & DD Loss-SCF V. ${ }^{\mathrm{a}}$ & -.29 & .51 & -.09 \\
3 & Age Group.-SCF V. & & & \\
& & -.48 & .21 & $-.35^{*}$
\end{tabular}

Note: $N=46$, SCF V. = Sunk-Cost Fallacy Vignette Score, DD Loss = Delay Discounting Loss Aversion Score, ${ }^{\mathrm{a} C o n t r o l l i n g ~ f o r ~ a g e ~ g r o u p . ~}{ }^{\mathrm{b}}$ Controlling for DD Loss, requirements based on Baron \& Kenny (1986) criteria, ${ }^{*} p<.05, * * p<.01, \dagger p<.001$. 
Table 12

Tradeoff Loss Aversion Task Score as a Mediator of the Relation Between Age Group and SunkCost Fallacy Vignette Score

Requirement no. $\quad$ Variable $\quad B \quad S E B \quad \beta$

\begin{tabular}{|c|c|c|c|c|}
\hline 1 & Age Group — SCF V. & -.36 & .12 & $-.28 * *$ \\
\hline $2 \mathrm{a}$ & Age Group_-Trade. Loss & -.002 & .00 & $-.44 \dagger$ \\
\hline $2 b$ & Trade. Loss-SCF V. ${ }^{\mathrm{a}}$ & .00 & .00 & .01 \\
\hline 3 & Age Group.-SCF V. ${ }^{b}$ & -.35 & .21 & $-.28 *$ \\
\hline
\end{tabular}

Note: $N=100$, SCF V. = Sunk-Cost Fallacy Vignette Score, Trade. Loss = Tradeoff Loss Aversion Task Score, ${ }^{\mathrm{a} C}$ Controlling for age group. ${ }^{\mathrm{b}}$ Controlling for Trade. Loss., requirements based on Baron \& Kenny (1986) criteria, ${ }^{*} p<.05, * * p<.01, \dagger p<.001$. 


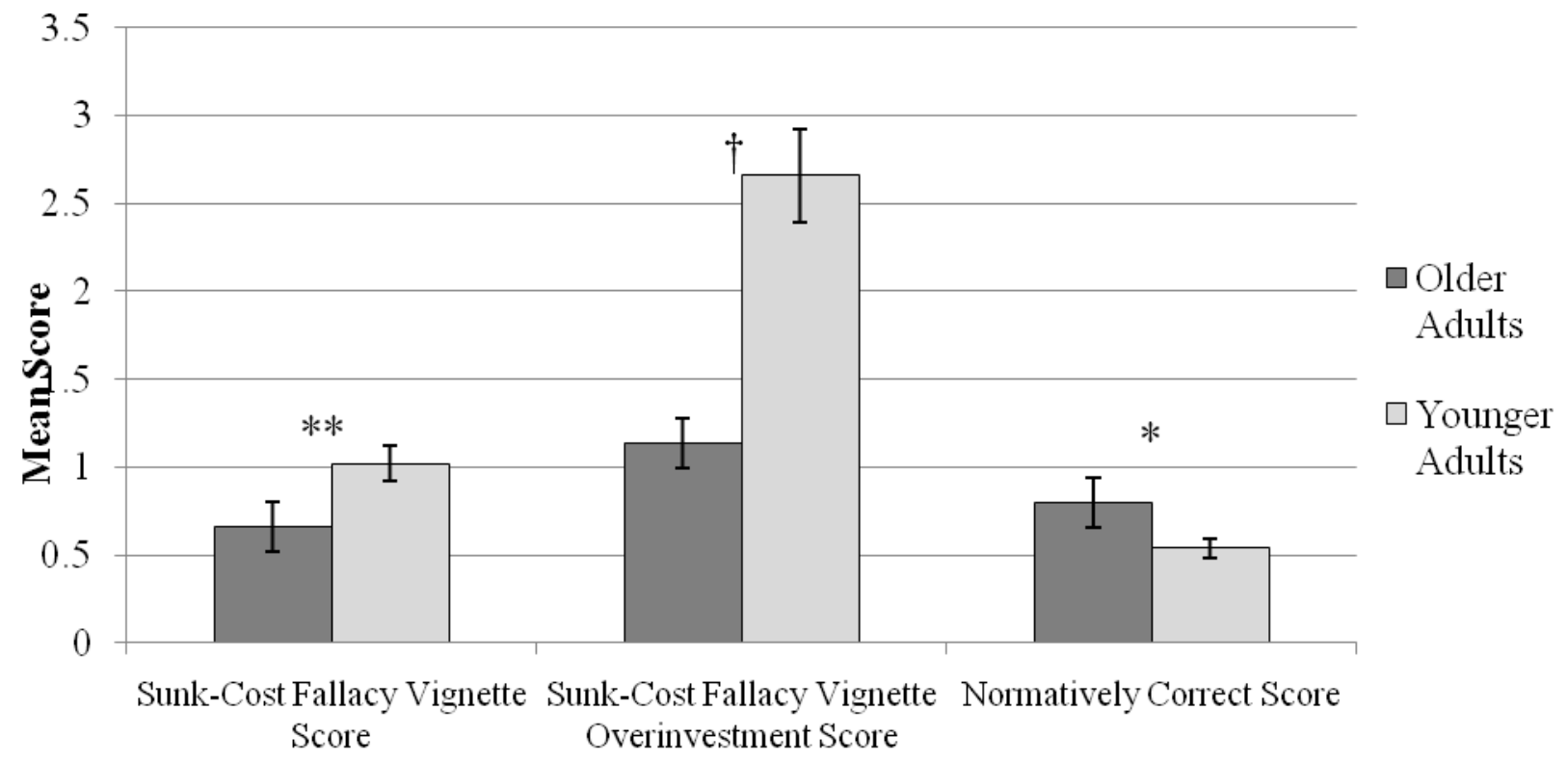

Measure

Figure 1. Age differences in Sunk-Cost Fallacy Vignette, Sunk-Cost Fallacy Vignette

Overinvestment, and Normatively Correct Scores. Note: ${ }^{*} p<.05, * * p<.01, \dagger p<.001$. 


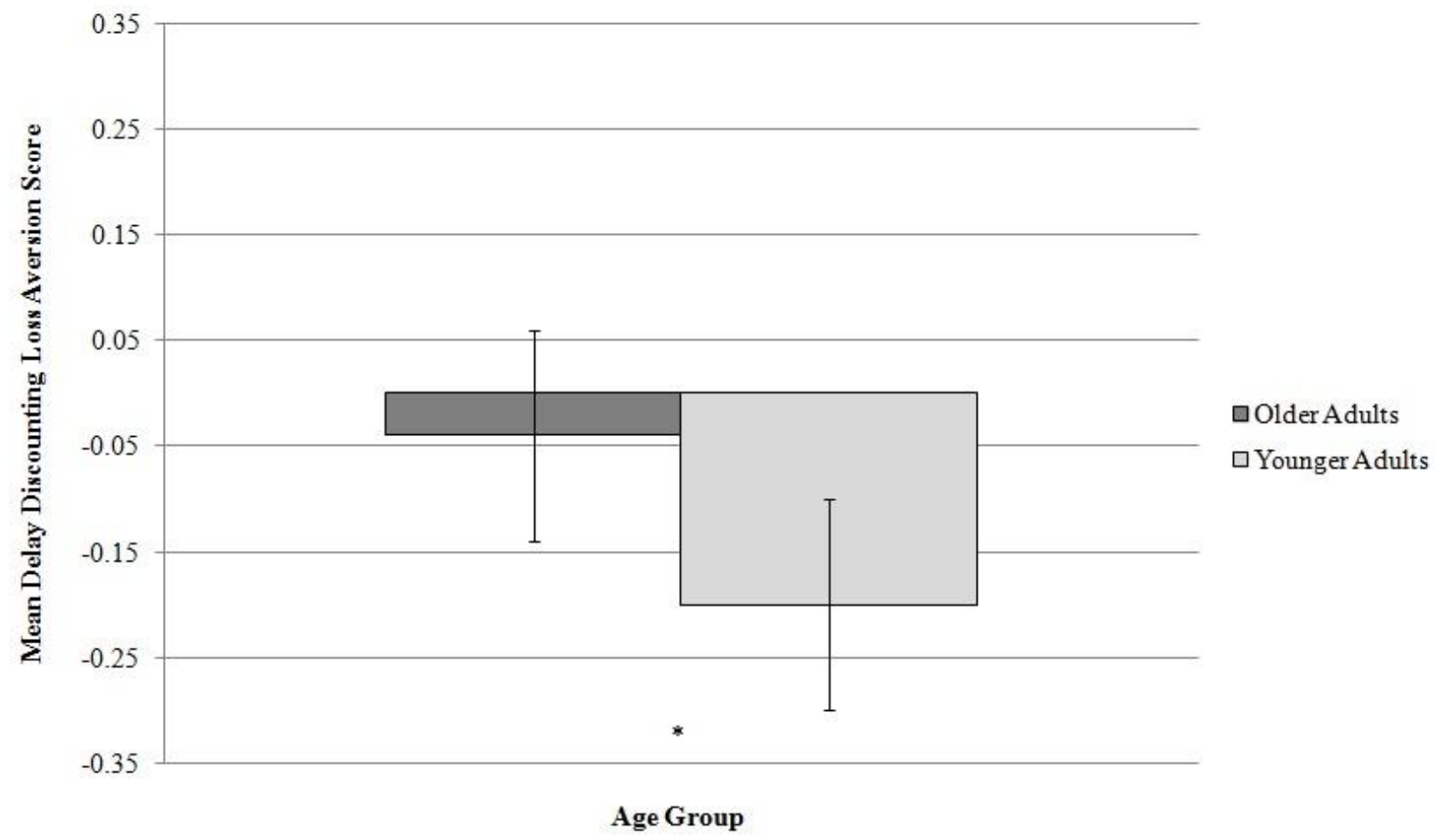

Figure 2. Age differences in Delay Discounting Loss Aversion Scores. Note: ${ }^{*} p<.05$. 


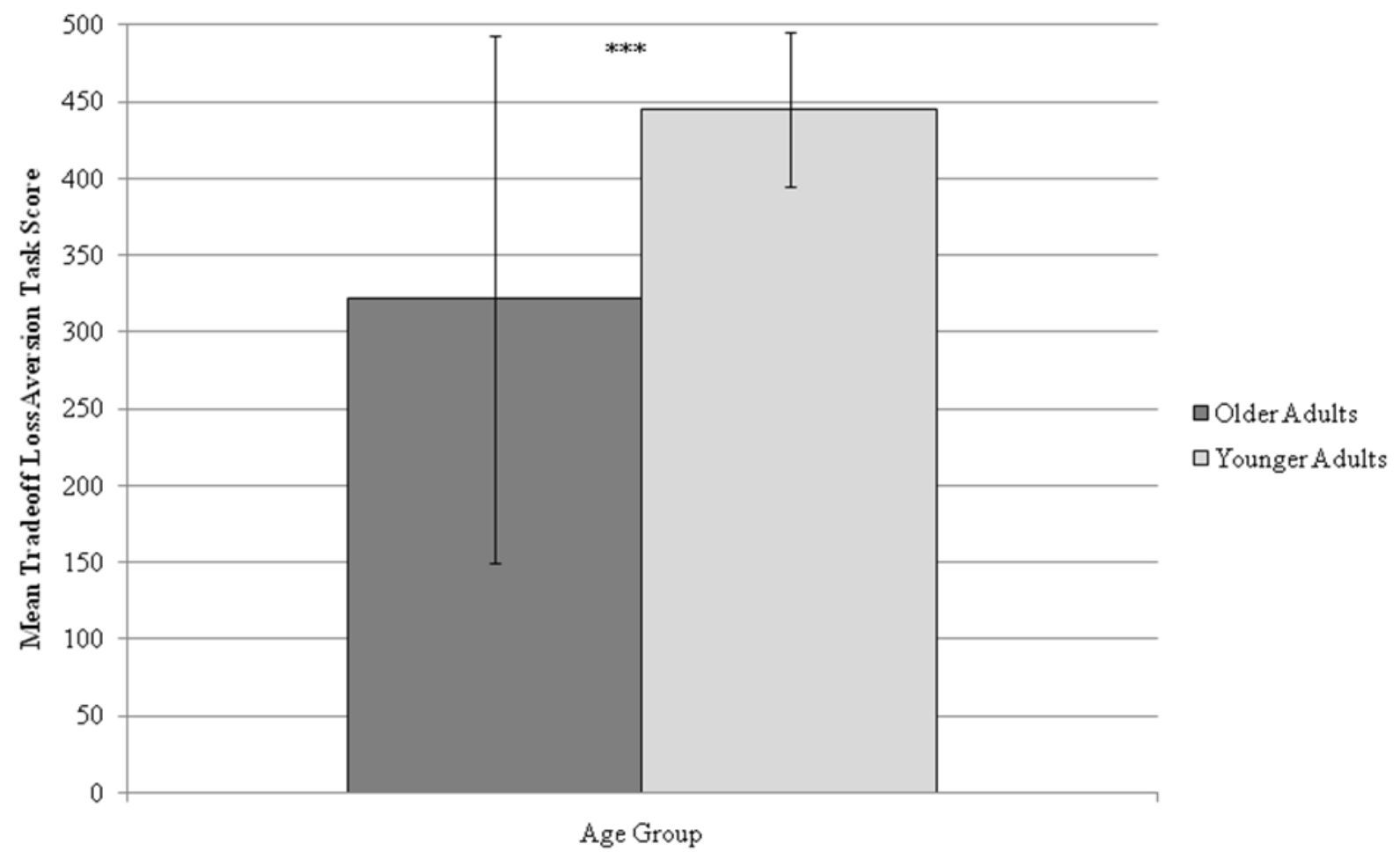

Figure 3. Age differences in Tradeoff Loss Aversion Task Scores. Note: ***p<.001. 


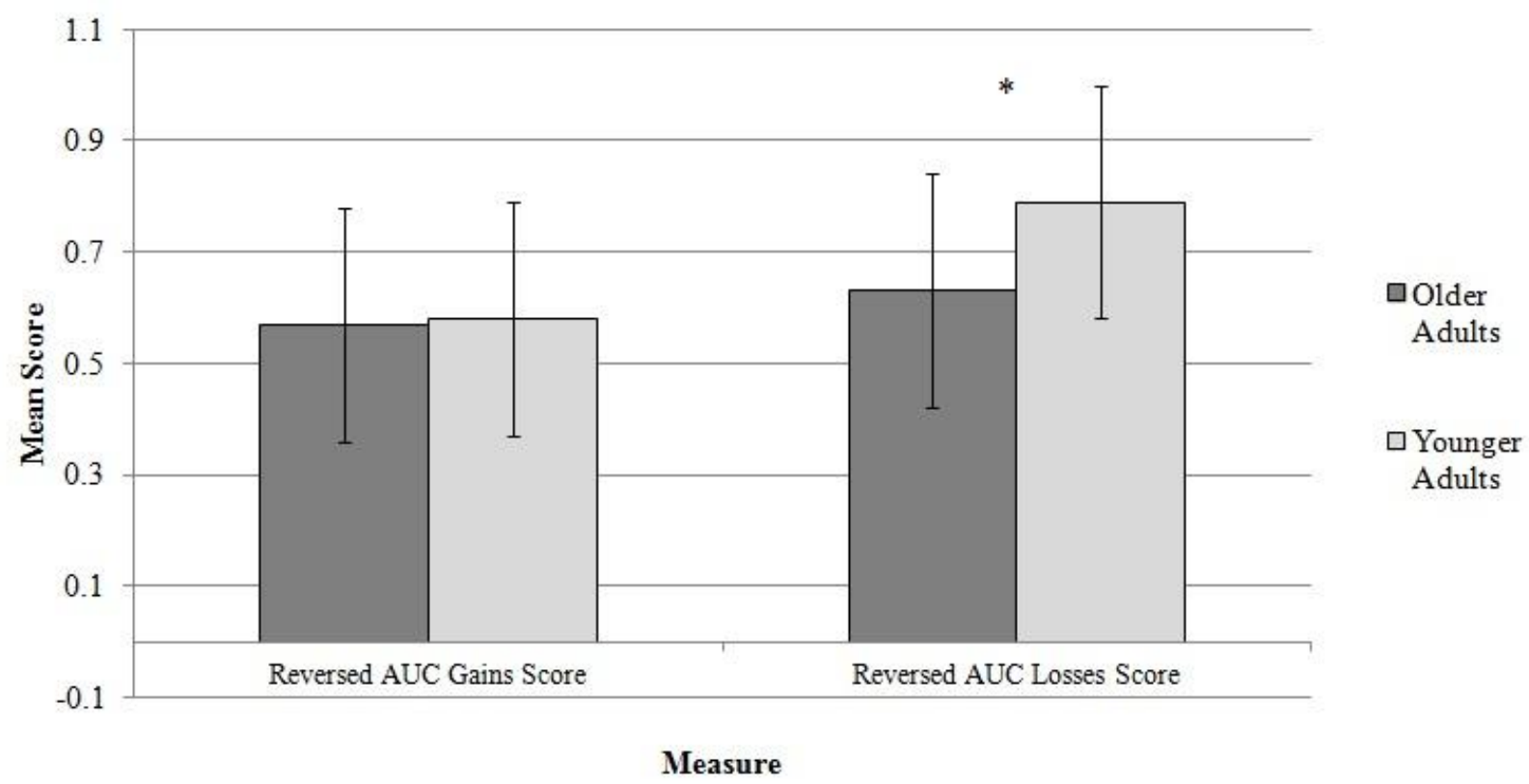

Figure 4. Age differences in Reversed AUC Gains and Losses Scores. Note: higher scores indicate greater discounting; $* p<.05$. 


\begin{tabular}{|c|c|c|c|}
\hline Delay & Delay (proportion of 25 years) & Normalized Subjective Value (Gains) & Area of Trapezoid \\
\hline 1 week & 0.000833333 & 0.83 & 0.00185 \\
\hline 1 month & 0.003333333 & 0.65 & 0.009416667 \\
\hline 6 months & 0.02 & 0.48 & 0.0078 \\
\hline 1 year & 0.04 & 0.30 & 0.018 \\
\hline 3 year & 0.12 & 0.15 & 0.0096 \\
\hline 5 year & 0.2 & 0.09 & 0.02 \\
\hline 10 year & 0.4 & 0.11 & 0.042 \\
\hline \multirow[t]{2}{*}{25 year } & 1 & 0.03 & \\
\hline & AUC Gains: & 0.108667 & \\
\hline Delay & Delay (proportion of 25 years) & Normalized Subjective Value (Losses) & Area of Trapezoid \\
\hline 1 week & 0.000833333 & 0.95 & 0.0021 \\
\hline 1 month & 0.003333333 & 0.73 & 0.01175 \\
\hline 6 months & 0.02 & 0.68 & 0.0121 \\
\hline 1 year & 0.04 & 0.53 & 0.0344 \\
\hline 3 year & 0.12 & 0.33 & 0.0244 \\
\hline 5 year & 0.2 & 0.28 & 0.038 \\
\hline 10 year & 0.4 & 0.10 & 0.045 \\
\hline \multirow[t]{2}{*}{25 year } & 1 & 0.05 & \\
\hline & AUC Losses: & 0.1677500 & \\
\hline
\end{tabular}

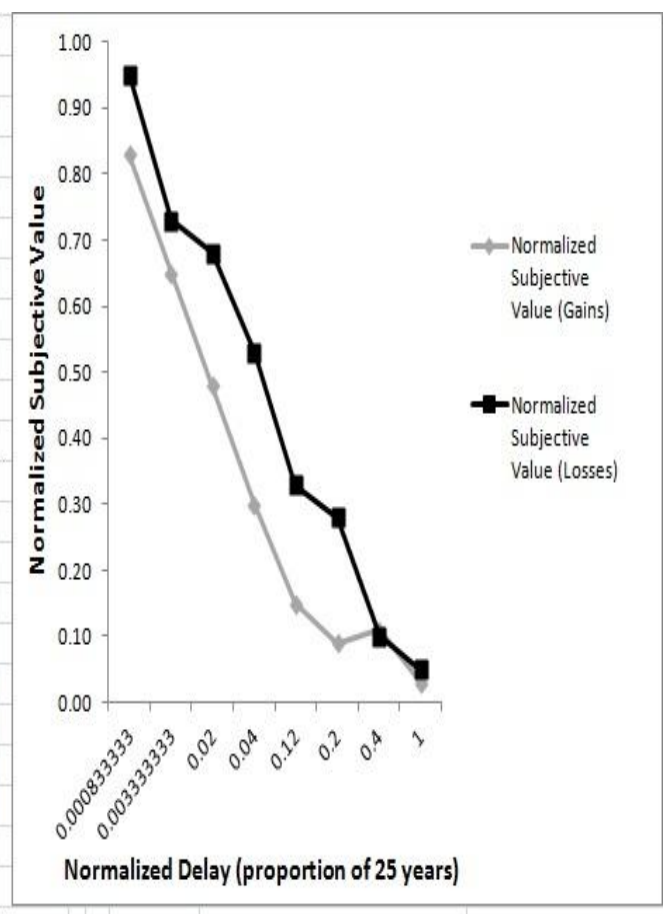

Delay Discounting Loss Aversion Score (AUC Losses - AUC Gains): $\quad 0.059083333$

Figure 5. Example illustration of graphed normalized subjective values used to calculate AUC Gains and Losses Scores and the Delay discounting Loss Aversion Score. Note: this figure is based on actual participant data. Normalized subjective values on the Y axis are the means of the switch points from the ascending and descending phases, divided by the fixed value (i.e., divided by 1000). Thus, on the Y axis, .10 represents a mean switch point of $\$ 100, .20$ equals a mean switch point of $\$ 200$, etc. Normalized delays on the $X$ axis are delay values that are a proportion of the maximum delay (i.e., 25 years), and correspond with the eight delay values depicted on the far left hand of the figure. 


\section{Appendix A}

\section{Calorie Estimation Task and Instructions}

Page 1 of Phase 1 Instructions as they appeared to participants:

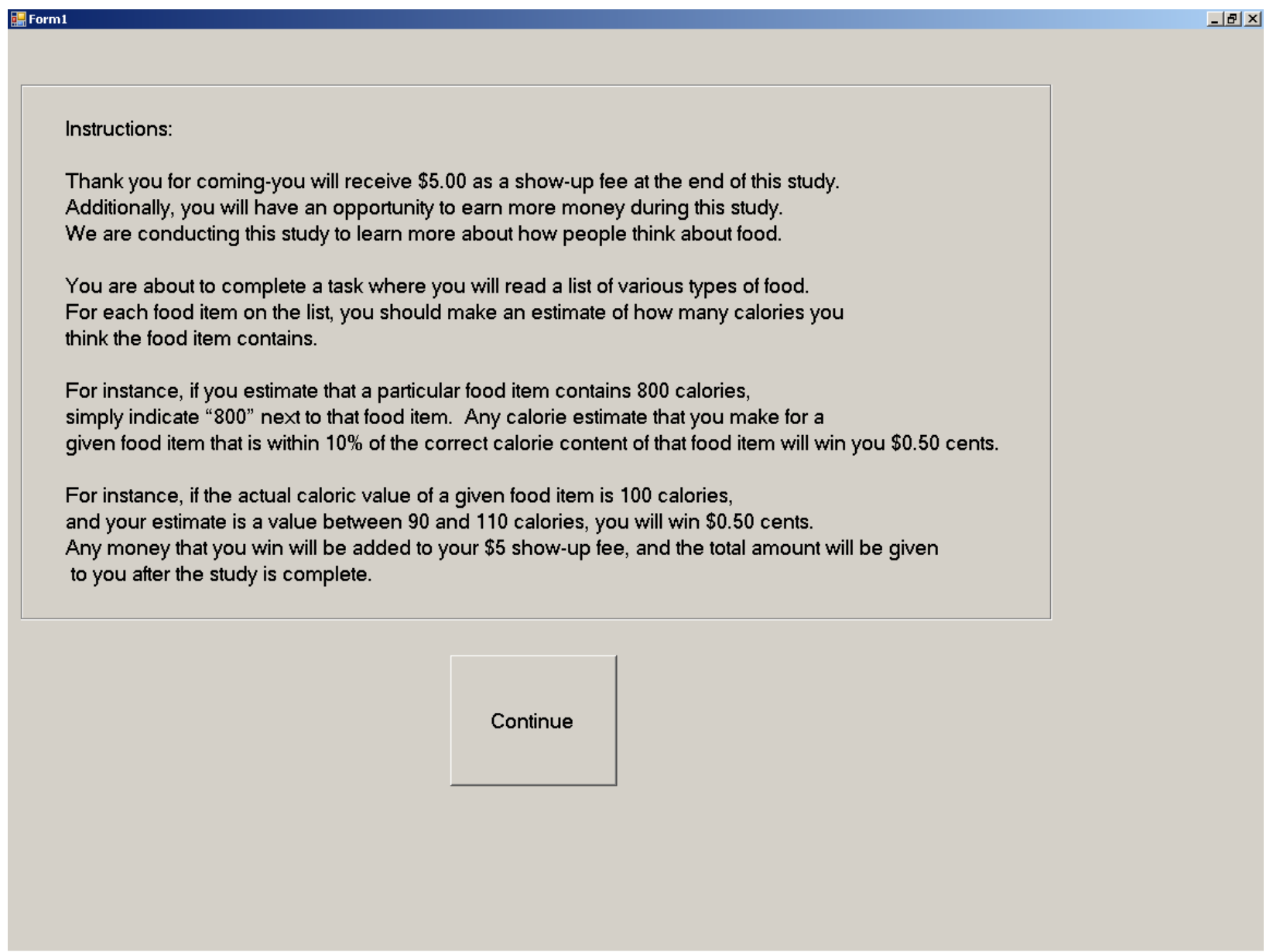


Page 2 of Phase 1 Instructions as they appeared to participants:

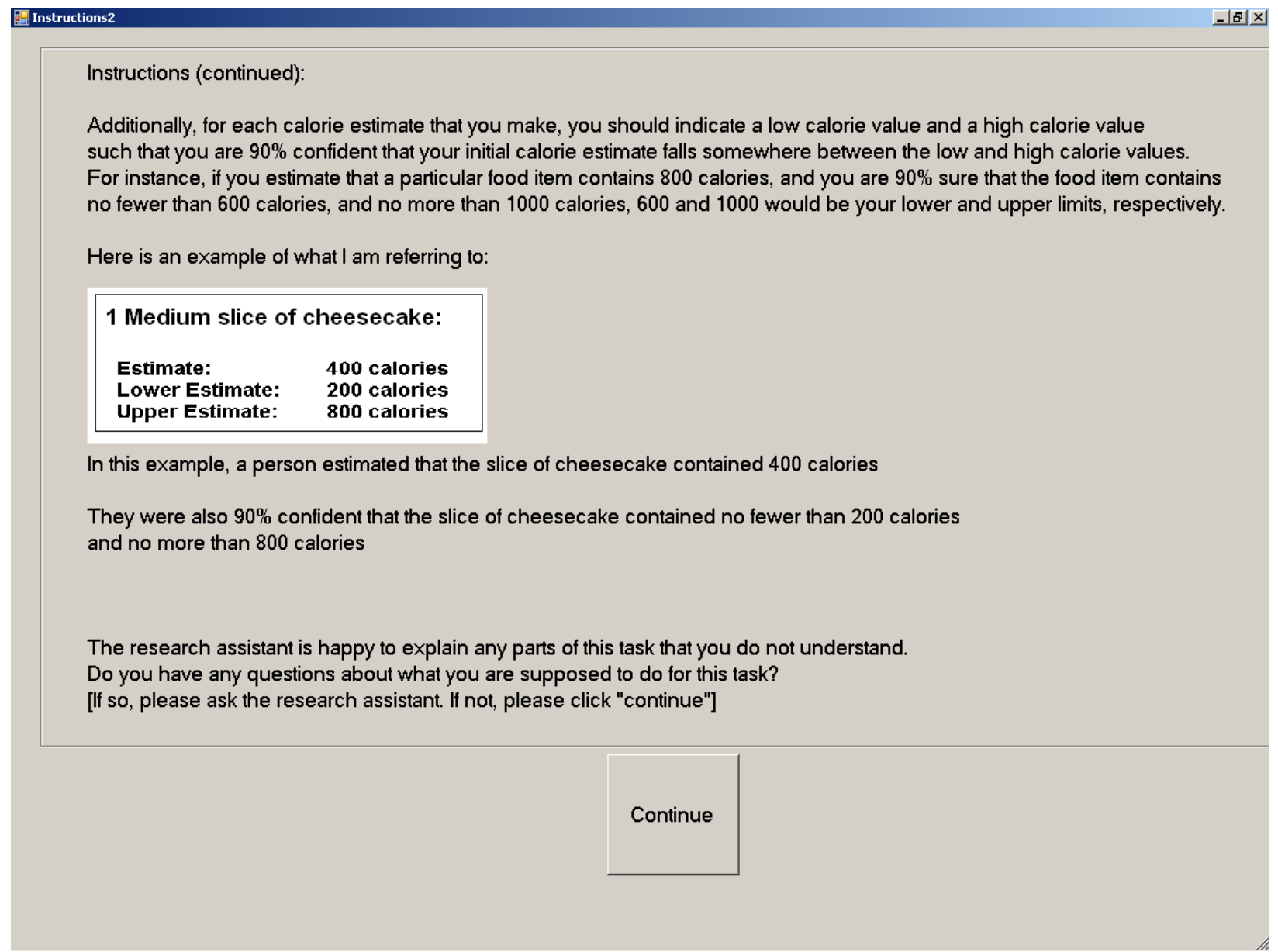


Calorie Estimation Task Phase 1 as it appeared to participants while completing Phase 1.

\section{區 Phase3}

You will win $\$ .50$ cents for each calorie estimate that is accurate (within $10 \%$ of the actual caloric value of the food item)

Food Item

1 package of Reese's Pieces® candies

1 serving (.99 oz) of Cheerios $₫$ cereal (with $1 / 2$ cup of skim milk)

1 ounce serving of Tostitos® restaurant -style tortilla chips

1 serving of Trix@ cereal (with $1 / 2$ cup of skim milk)

1 regular sized Kit-Kat ${ }^{\circledR}$ candy bar

2 cups ( 1 can) of Progresso® traditional beef_barley soup

Lean Cuisine $\AA^{\circledR}$ chicken and vegetables microwavable dinner (10.5 oz)

15 Wheat Thins $®$ crackers (tomato_basil flavored)

1 Milkyway ${ }^{\circledR}$ candy bar (dark chocolate 1.9oz)

One 12-ounce can of regular Coca-Cola®

1 regular sized $(1.37 \mathrm{oz})$ Skor $₫$ chocolate_toffee candy bar

1 serving (1/2 cup) of Breyer's® chocolate ice cream

1 serving (1/2 cup) of Campbell's $₫$ Chicken with Rice Soup

1 slice of Pepperidge Farm ${ }^{\circledR}$ seven grain bread

1 ounce serving of Cheetos $\circledast$ cheddar jalapeno-flavored snacks

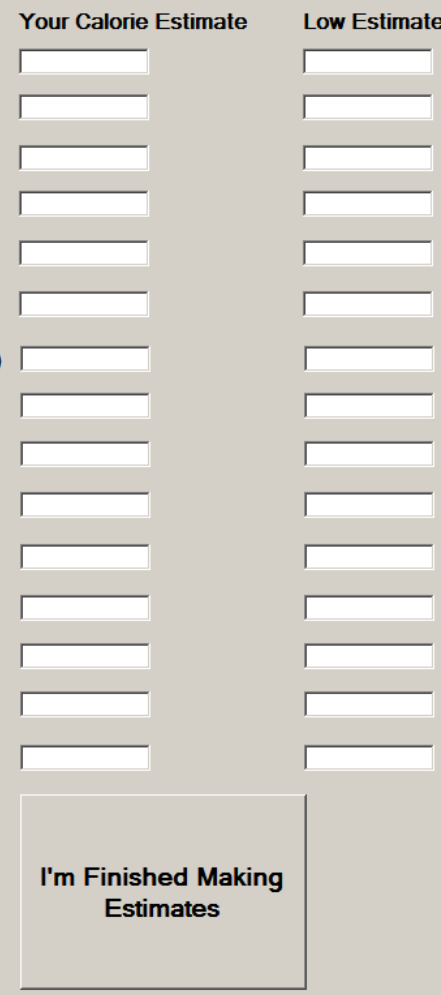

High Estimate
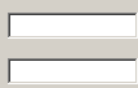

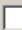
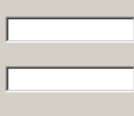

$+$

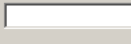

$+$

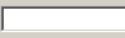

$+2$

$\square$

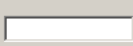

$+$

$+$

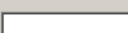


Phase 2 instructions as they appeared to participants after completing Phase 1. The amount of advice cost described in these instructions-small (\$1.00) vs. large (\$3.00)—was counterbalanced across sequences.

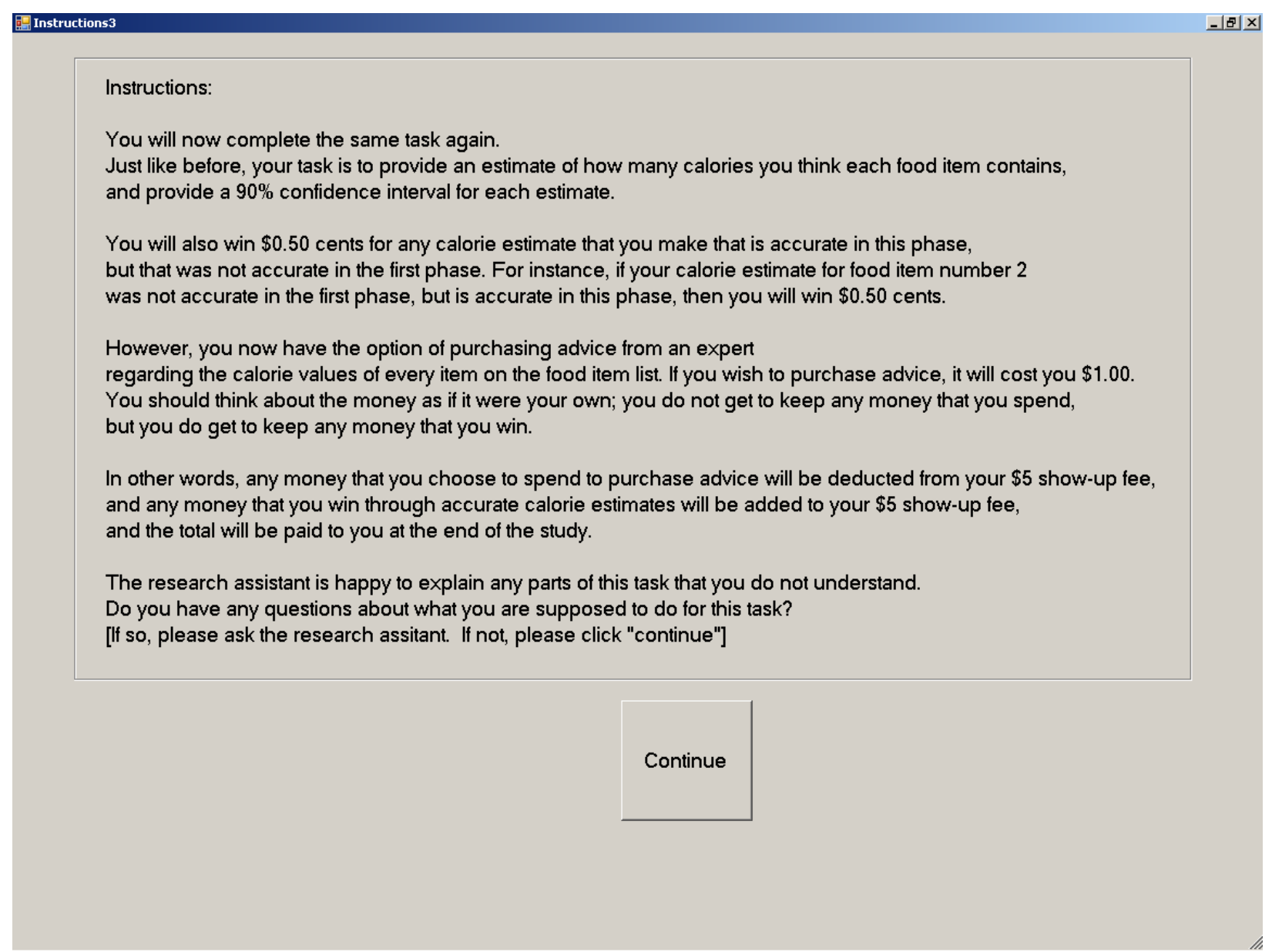


Calorie Estimation Task Phase 2 as it appeared to participants. The price of advice on the "Purchase Advice” button—small (\$1.00) vs. large (\$3.00)—was counterbalanced across sequences.

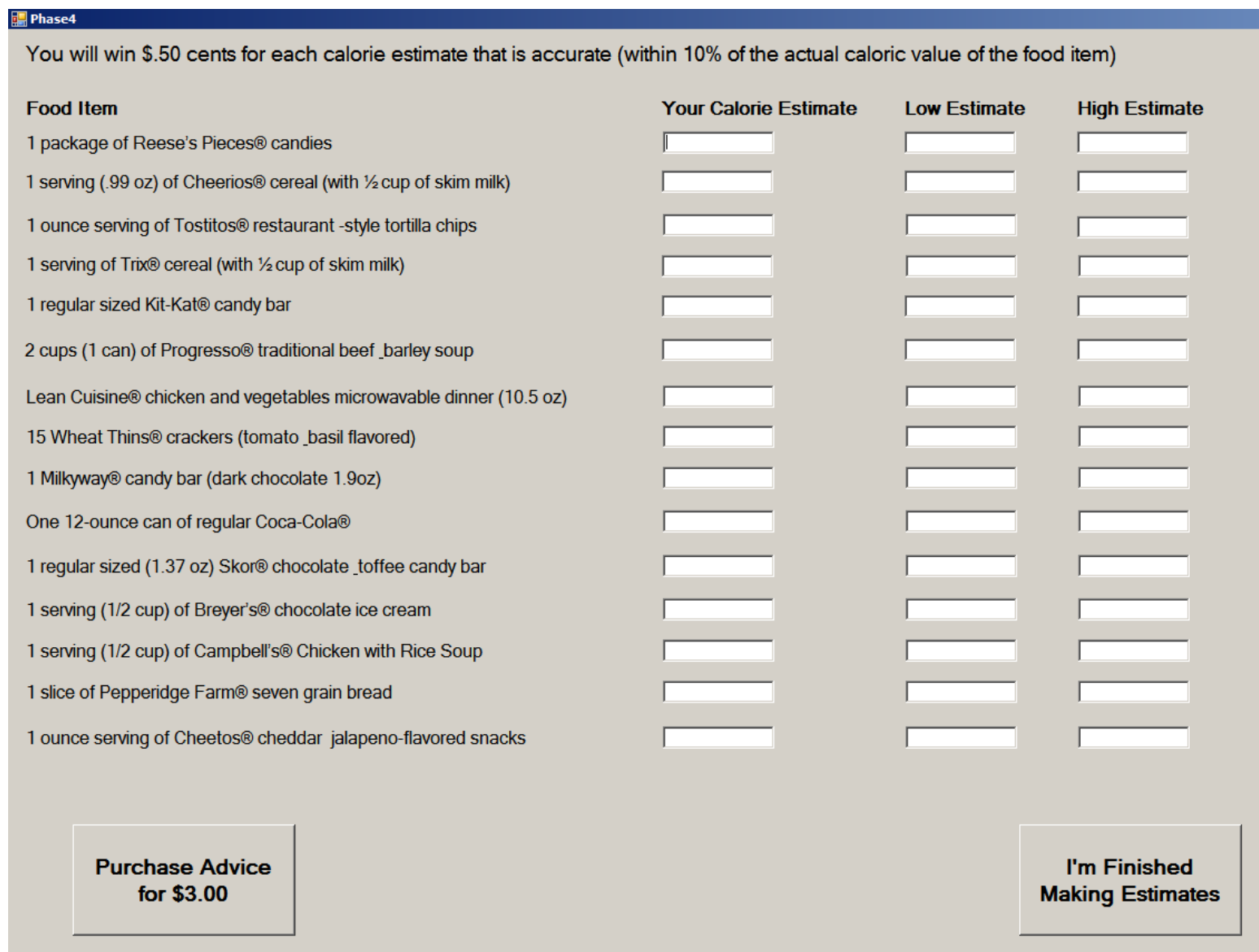


Calorie Estimation Task Phase 2 as it appeared to participants after purchasing advice. The price of advice on the "Purchase Advice" button-small (\$1.00) vs. large (\$3.00)—was counterbalanced across sequences, as was the dollar amount in the "You have Purchased Advice” message.

\begin{tabular}{|c|c|c|c|c|}
\hline \multicolumn{5}{|l|}{ Phase4 } \\
\hline \multicolumn{5}{|c|}{50 cents for each calorie estimate that is accurate (within $10 \%$ of the actual caloric value of the food item) } \\
\hline Food Item & Advice & Your Calorie Estimate & Low Estimate & High Estimate \\
\hline 1 package of Reese's Pieces ${ }^{\circledR}$ candies & 195 & & & \\
\hline 1 serving ( $.99 \mathrm{oz}$ ) of Cheerios $\circledast$ cereal (with $1 / 2$ cup of skim milk) & 90 & & & \\
\hline 1 ounce serving of Tostitos $\circledast$ restaurant -style tortilla chips & 105 & & & \\
\hline 1 serving of Trix $\circledast$ cereal (with $1 / 2$ cup of skim milk) & 130 & & & \\
\hline 1 regular sized Kit-Kat@ candy bar & 205 & & & \\
\hline 2 cups ( 1 can) of Progresso® traditional beef_barley soup & 210 & & & \\
\hline Lean Cuisine $₫$ chicken and vegetables microwavable dinner ( $10.5 \mathrm{oz}$ ) & 150 & & & \\
\hline 15 Wheat Thins $₫$ crackers (tomato_basil flavored) & 105 & & & \\
\hline 1 Milkyway® candy bar (dark chocolate $1.90 z$ ) & 180 & & & | \\
\hline One 12-ounce can of regular Coca-Cola® & 145 & & & ए \\
\hline 1 regular sized $(1.37 \mathrm{oz})$ Skor@ chocolate _toffee candy bar & 200 & & & \\
\hline 1 serving ( $1 / 2$ cup) of Breyer's $\circledast$ chocolate ice cream & 165 & & & 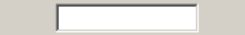 \\
\hline 1 serving ( $1 / 2$ cup) of Campbell's $₫$ Chicken with Rice Soup & 95 & & & 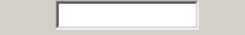 \\
\hline 1 slice of Pepperidge Farm $\circledast$ seven grain bread & 72.5 & & & 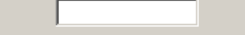 \\
\hline 1 ounce serving of Cheetos ${ }^{\circledR}$ cheddar jalapeno-flavored snacks & 125 & & & 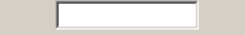 \\
\hline $\begin{array}{c}\text { Purchase Advice } \\
\text { for } \$ 3.00\end{array}$ & purchas & sed advice for $\$ 3.00$ & & $\begin{array}{c}\text { I'm Finished } \\
\text { Making Estimates }\end{array}$ \\
\hline
\end{tabular}


Quality of Advice Measure as it appeared to participants who purchased advice (This measure appeared following either Phase 3 or Phase 4 only if the participant purchased advice in that respective phase).

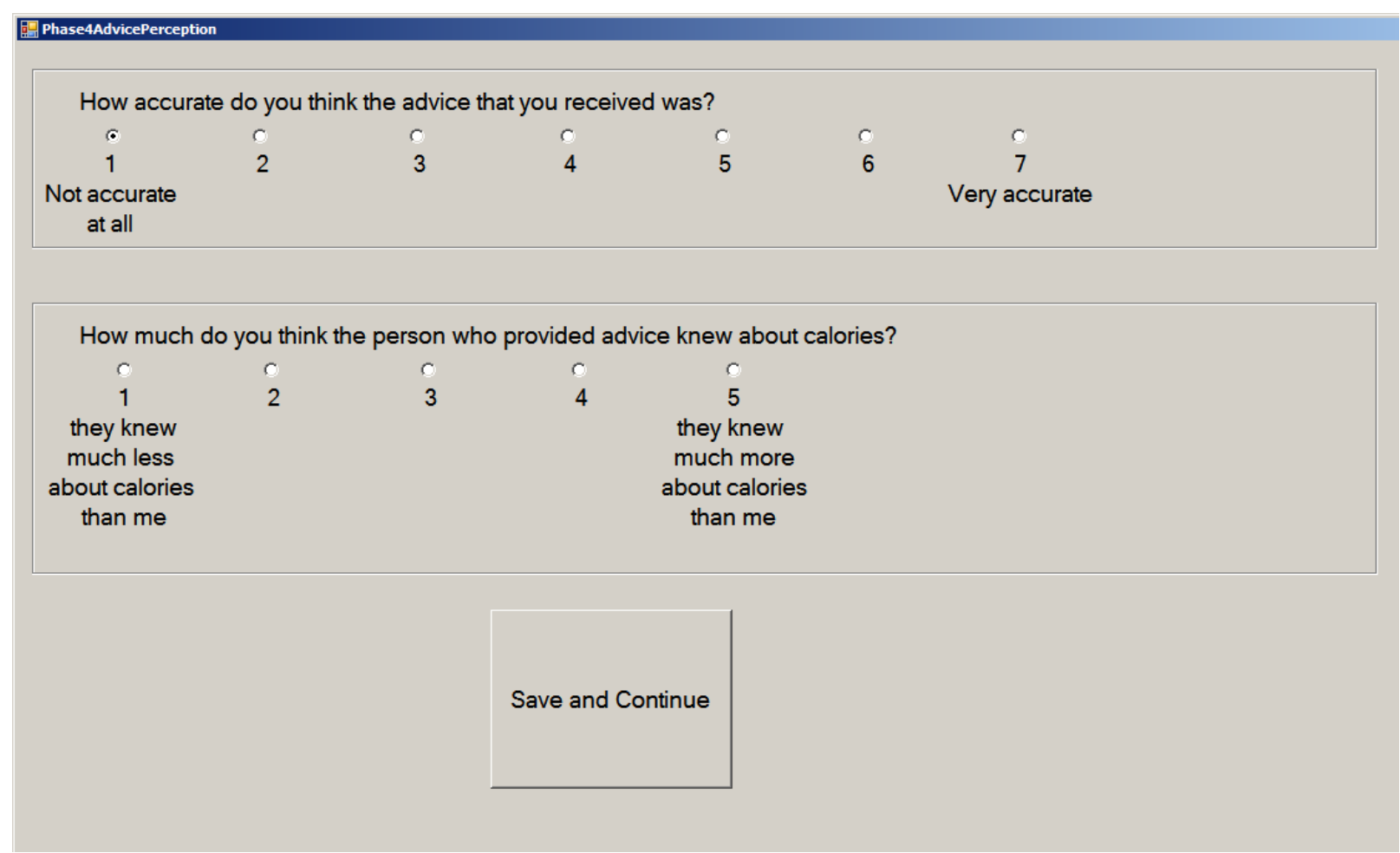


Phase 3 instructions as they appeared to participants:

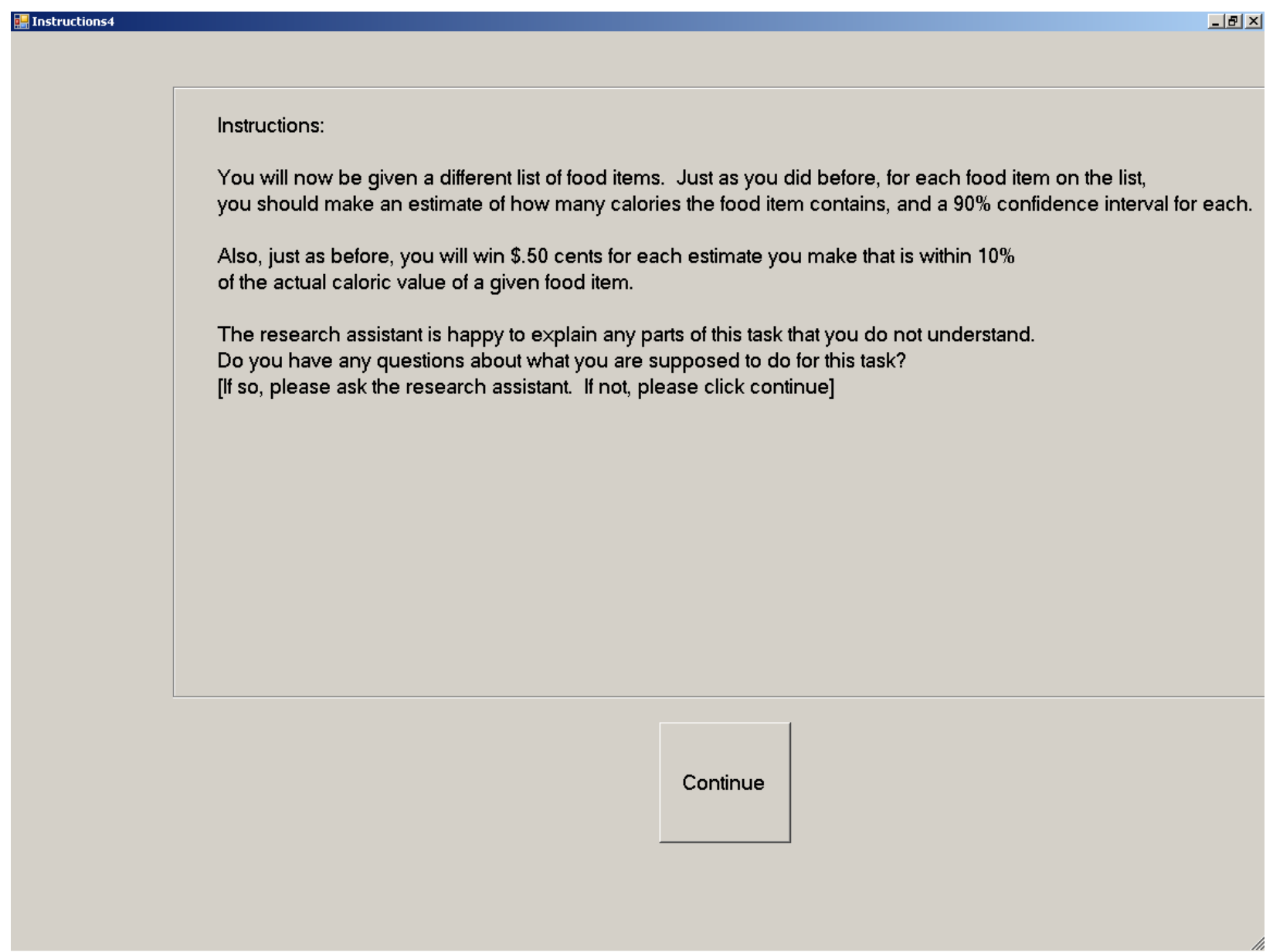

Phase 4 instructions were identical to Phase 2 instructions, except that the amount of money required to purchase advice was different (i.e., small/large price of advice). 


\section{Appendix B}

Food Item Lists 1 and 2

Food items with a number "1" appeared on Food Item List 1, whereas food items with a number "2" appeared on Food Item List 2. Each food item on List 1 had a matched food item on List 2 which was similar in terms of type of food, quantity, and caloric value. The values under "Actual" are the actual caloric values of each food item. The values under "Est." are the estimates obtained from a nutritionist through food item pilot testing. The bolded values under "Mean" are the means of the nutritionist's estimates for each of the analogous food items; these were the values that were provided to participants who purchased advice.

Food Item

1. 1 package of $M \& M ' s \circledR$ plain chocolate candies

2. 1 package of Reese's Pieces ${ }^{\circledR}$ candies
Calories

Actual Est. Mean

$240 \quad 200 \quad \mathbf{1 9 5}$

$210 \quad 190$

$\begin{array}{lll}140 \quad 80 & 90\end{array}$

$140 \quad 100$

$140 \quad 100 \quad \mathbf{1 0 5}$

$140 \quad 110$

$150 \quad 110$

130

$160 \quad 150$

210200

205

$210 \quad 210$

2. 1 regular sized Kit-Kat® candy bar 
1. 2 cups $(1$ can $)$ of Progresso $®$ traditional beef $\&$ vegetable soup

2. 2 cups ( 1 can) of Progresso $®$ traditional beef $\&$ barley soup

1. Lean Cuisine ${ }^{\circledR}$ chicken with almonds microwavable dinner $(10.5 \mathrm{oz})$

2. Lean Cuisine ${ }^{\circledR}$ chicken and vegetables microwavable dinner $(10.5 \mathrm{oz})$

1. 15 Wheat Thins ${ }^{\circledR}$ crackers (ranch flavored)

2. 15 Wheat Thins $®$ crackers (tomato \& basil flavored)

1. 1 Milkyway ${ }^{\circledR}$ candy bar (milk chocolate $1.9 \mathrm{oz}$ )

2. 1 Milkyway® candy bar (dark chocolate $1.9 \mathrm{oz}$ )

1. One 12-ounce can of regular Pepsi®

2. One 12-ounce can of regular Coca-Cola ${ }^{\circledR}$

1. 1 regular sized $(1.37 \mathrm{oz})$ Heath ${ }^{\circledR}$ chocolate $\&$ toffee candy bar

2. 1 regular sized $(1.37 \mathrm{oz})$ Skor $®$ chocolate \& toffee candy bar

1. 1 serving ( $1 / 2$ cup) of Breyer's ${ }^{\circledR}$ vanilla ice cream

2. 1 serving ( $1 / 2$ cup) of Breyer's ${ }^{\circledR}$ chocolate ice cream

1. 1 serving ( $1 / 2$ cup) of Campbell's ${ }^{\circledR}$ Chicken Vegetable Soup

2. 1 serving ( $1 / 2$ cup) of Campbell's ${ }^{\circledR}$ Chicken with Rice Soup
$240 \quad 240$

$240 \quad 180$

250

150

150

240

150

140

90

105

140

120

228

190

180

$224 \quad 170$

150

110

145

140

180

210

180

200

200

220

130

180

165

140

150

80

110

95

70 
1. 1 slice of Pepperidge Farm ${ }^{\circledR} 100 \%$ whole wheat bread

2. 1 slice of Pepperidge Farm ${ }^{\circledR}$ seven grain bread

1. 1 ounce serving of Cheetos $₫$ cheese-flavored snacks

2. 1 ounce serving of Cheetos® cheddar jalapeno-flavored snacks $\begin{array}{lll}110 & 75 & \mathbf{7 2 . 5}\end{array}$

$110 \quad 70$

$160 \quad 150 \quad \mathbf{1 2 5}$

$170 \quad 100$ 


\section{Appendix C}

\section{Delay Discounting of Gains and Losses Task}

First page of instructions, as they appeared to participants:

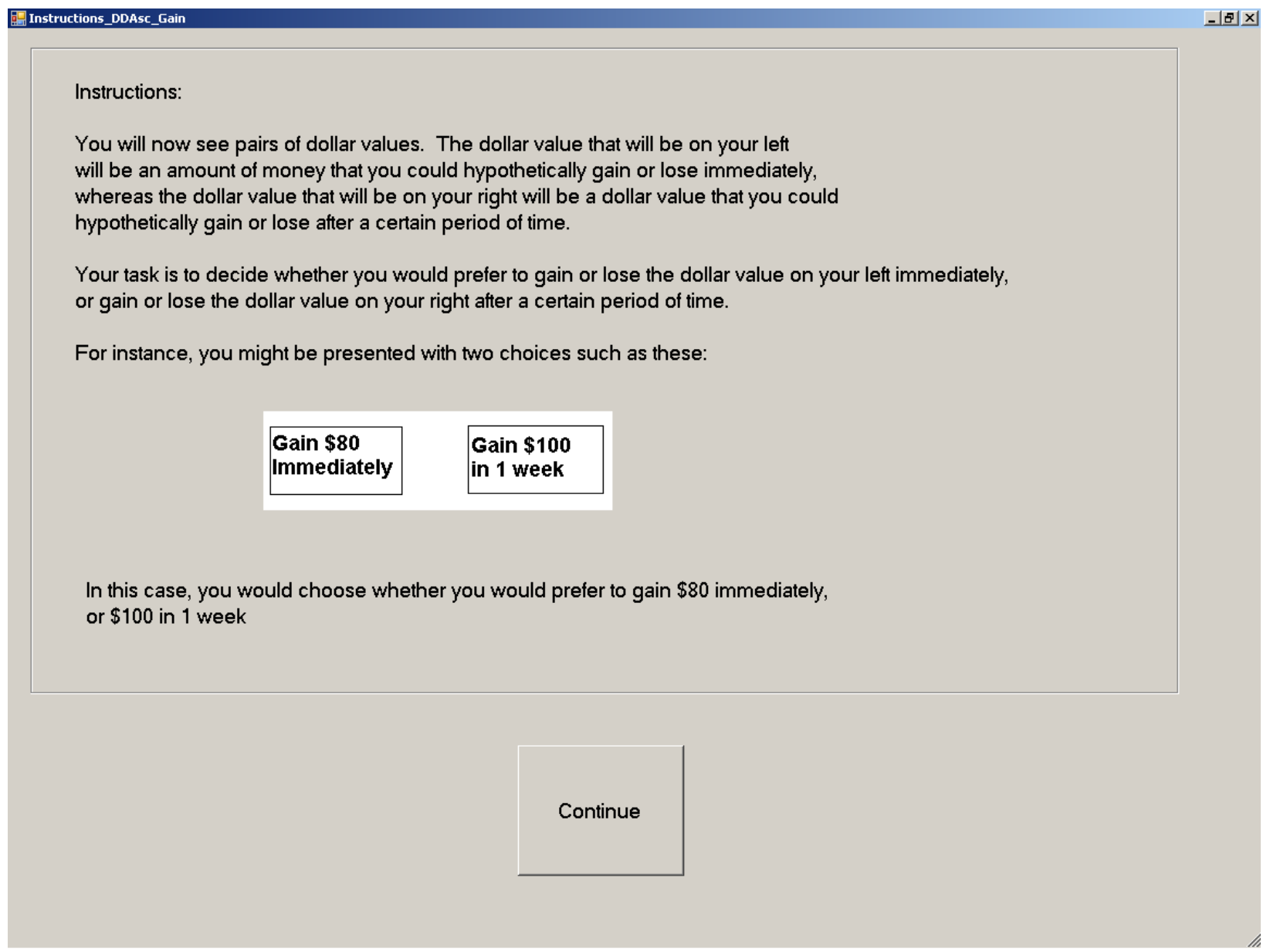


Second page of instructions, as they appeared to participants:

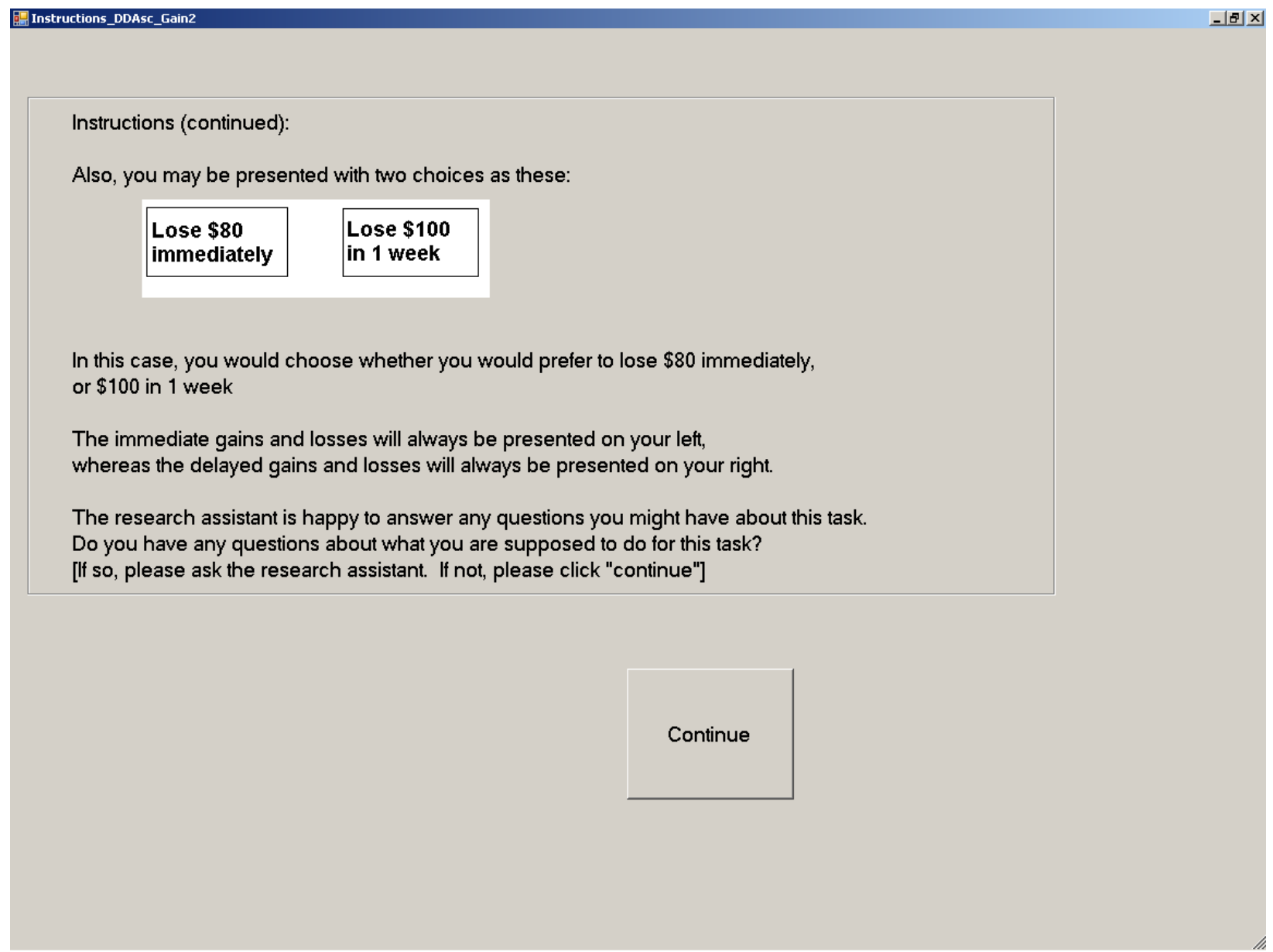


The values on the left and right were presented simultaneously to each participant. After making their choice, the value on the left was changed by computer program to the next value on this list.

The immediate gain/loss values were presented in ascending order, and also in descending order. The order of presentation was counterbalanced across participants.

In the gains condition, all dollar values were presented as monetary gains, whereas they were presented as monetary losses in the losses condition.

The time of delay was changed across each of the 16 trials within the gains condition and also within the losses condition. Each trial used a time delay of either 1 week, 1 month, 6 months, 1 year, 3 years, 5 years, 10 years, and 25 years.

Gain (lose) this value immediately:

$\$ 1$

$\$ 5$

$\$ 10$

$\$ 20$

$\$ 40$

$\$ 60$

$\$ 80$

$\$ 100$

$\$ 150$

$\$ 200$

$\$ 250$

$\$ 300$
Gain (lose) \$1000 in...

1 week

1 week

1 week

1 week

1 week

1 week

1 week

1 week

1 week

1 week

1 week

1 week 
$\$ 350$

$\$ 400$

$\$ 450$

$\$ 500$

$\$ 550$

$\$ 600$

$\$ 650$

$\$ 700$

$\$ 750$

$\$ 800$

$\$ 850$

$\$ 900$

$\$ 920$

$\$ 940$

$\$ 960$

$\$ 980$

$\$ 990$

$\$ 1000$
1 week

1 week

1 week

1 week

1 week

1 week

1 week

1 week

1 week

1 week

1 week

1 week

1 week

1 week

1 week

1 week

1 week

1 week 
Example screen shot of first trial of gains condition, ascending order:

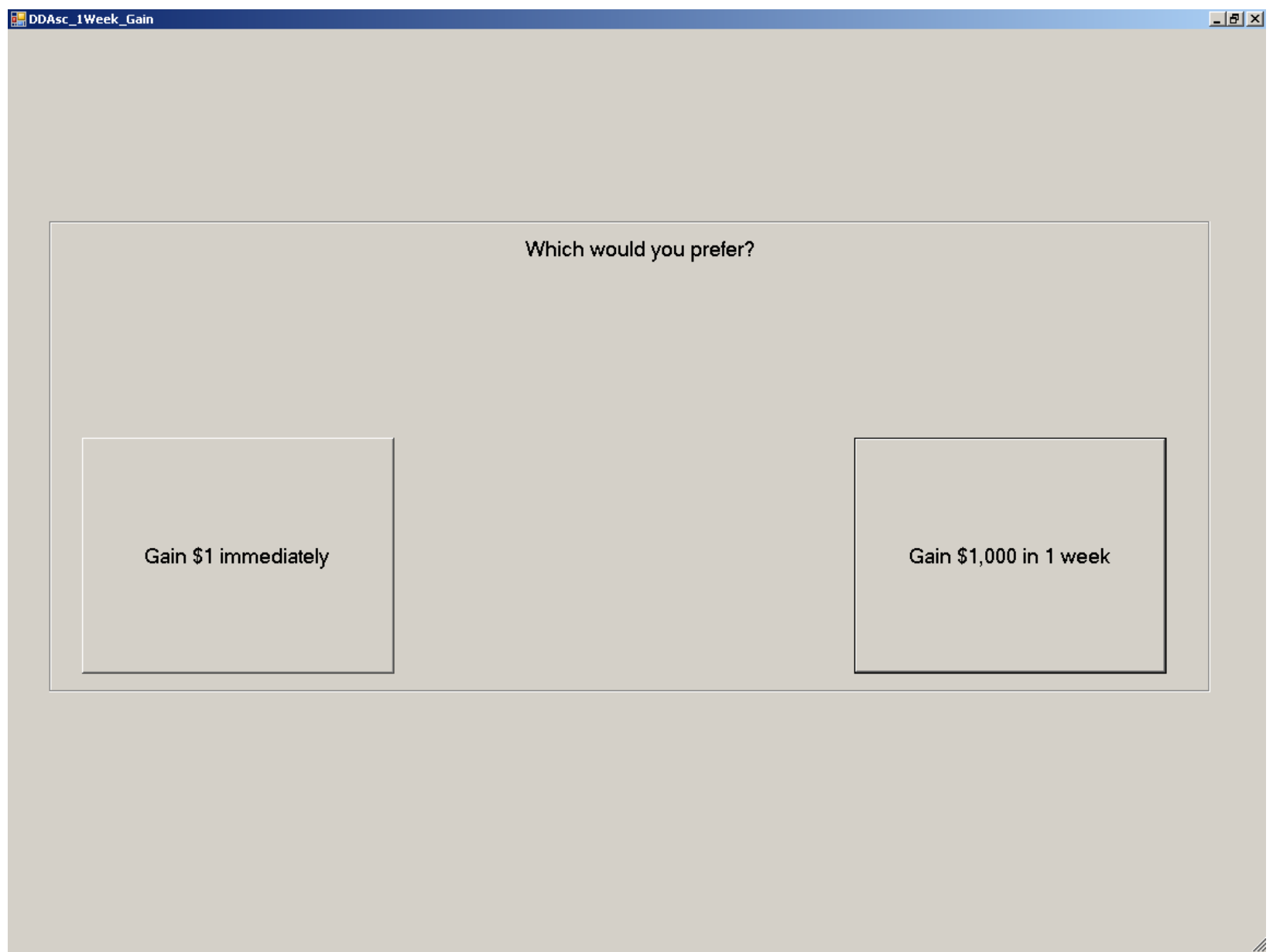


Example screenshot of first trial of gains condition, descending order:

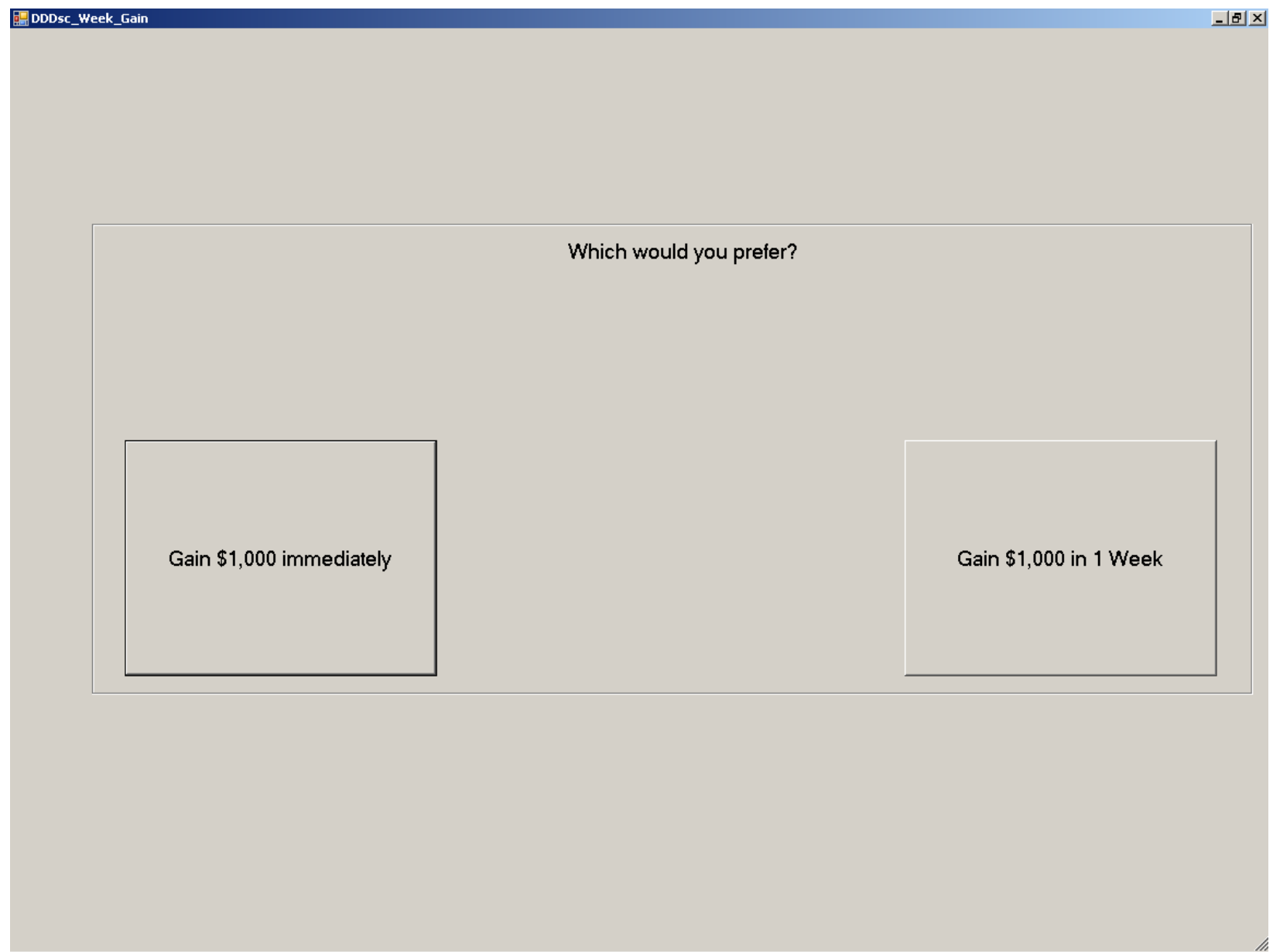


Example screenshot of first trial of losses condition, ascending order:

Which would you prefer?
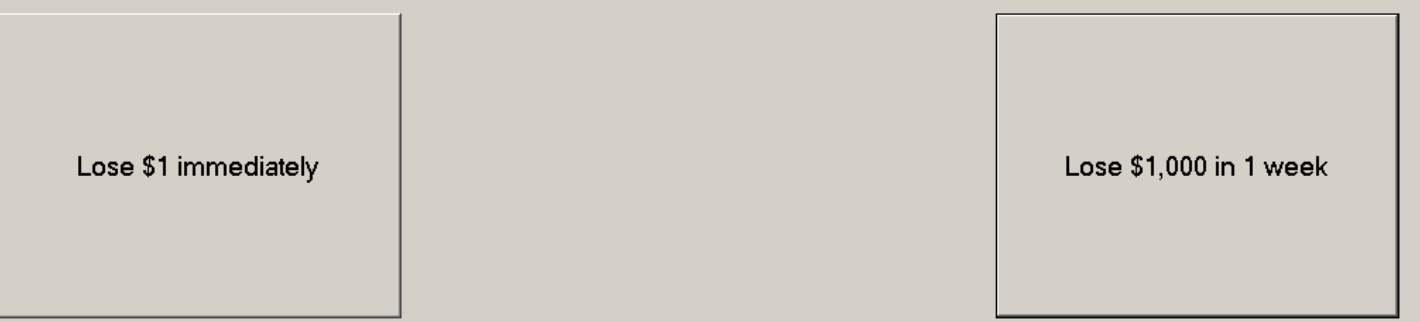
Example screenshot of first trial of losses condition, ascending order:

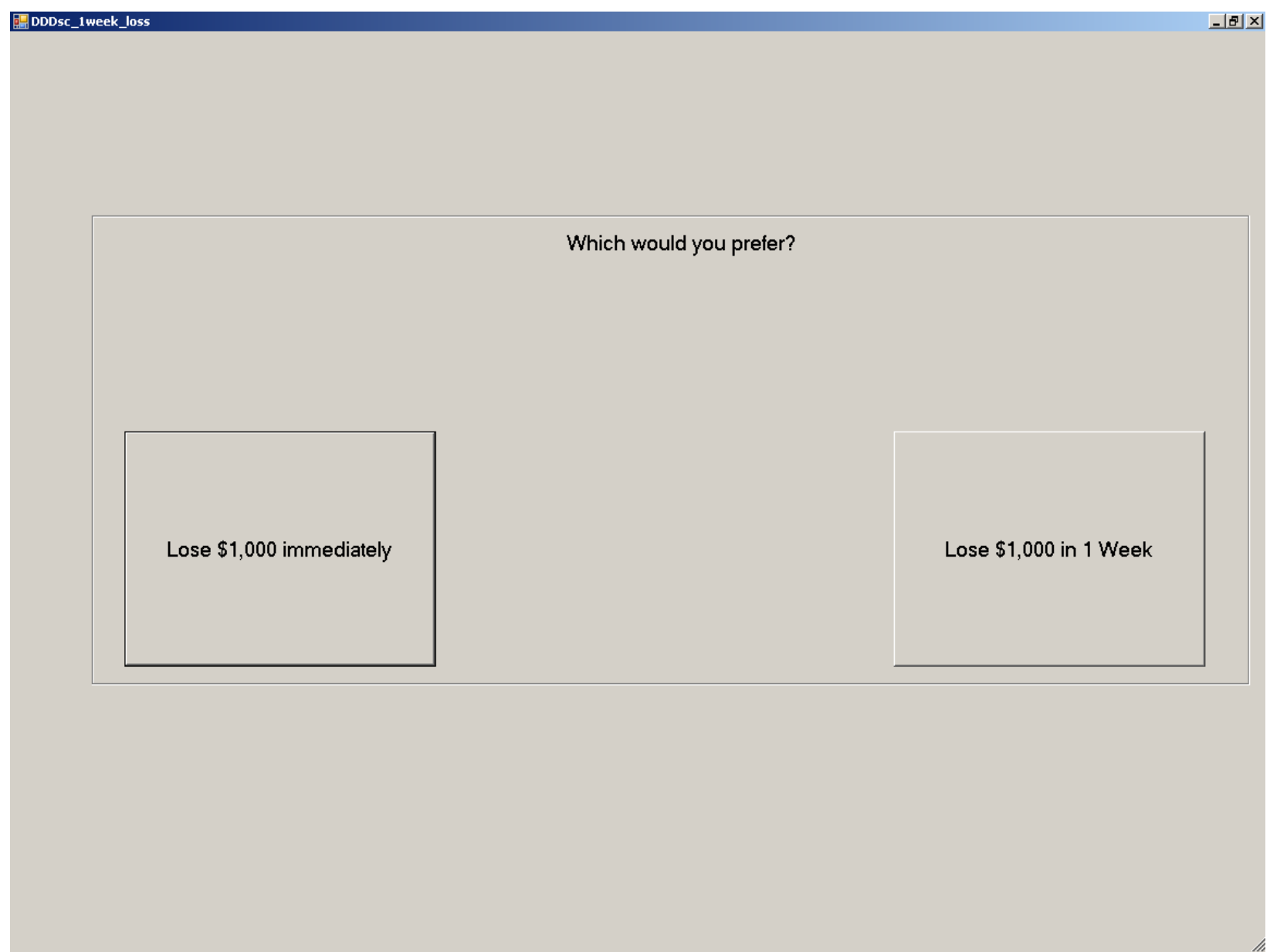




\section{Appendix D}

Tradeoff Task

Page 1 of Instructions as they appeared to participants:

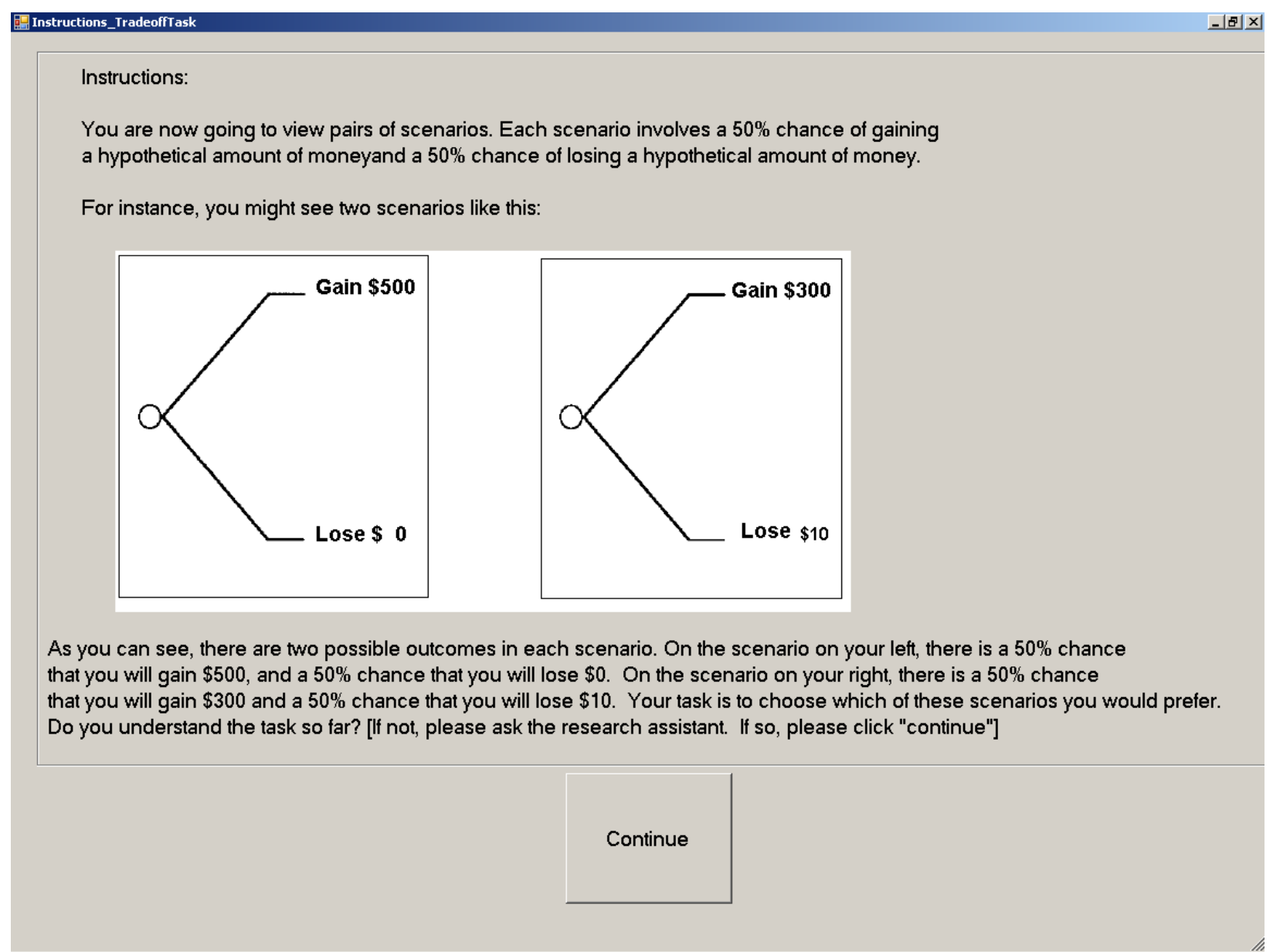


Page 2 of Instructions, as they appeared to participants:

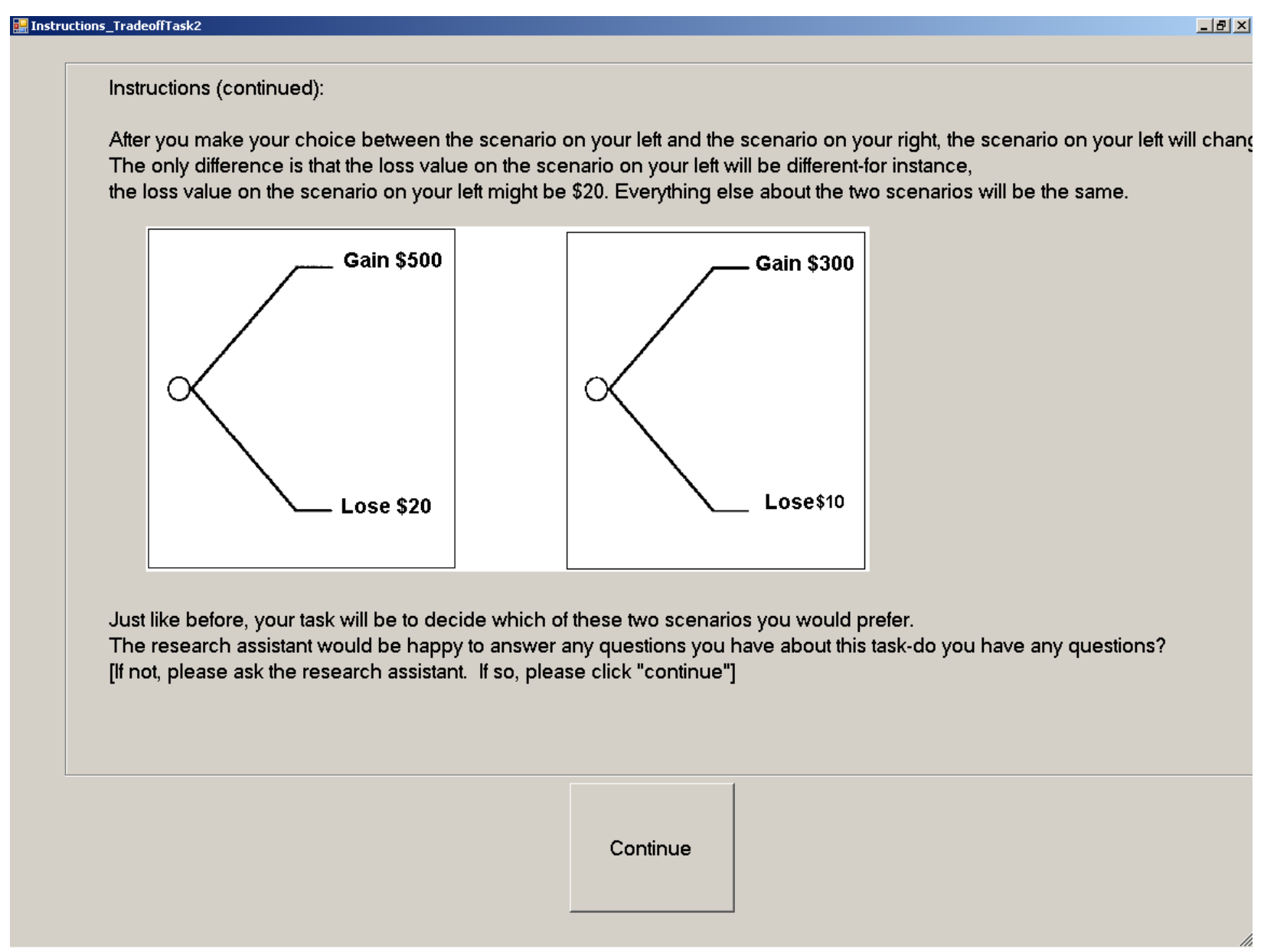


Example screenshot of first session of the Tradeoff Task, as it appeared to participants. Loss values on the button on the left increased by $\$ 5$ increments from trial to trial (i.e., as the participant clicked the button on the left) in all sessions until the participant reached their "indifference point" (i.e., until they clicked the button on the right), at which time the session ended and a new session began. Gain values remained the same across all sessions. Initial loss values were as follows: Session 1: \$25, Session 2: \$50, Session 3: \$75, Session 4: $\$ 100$.

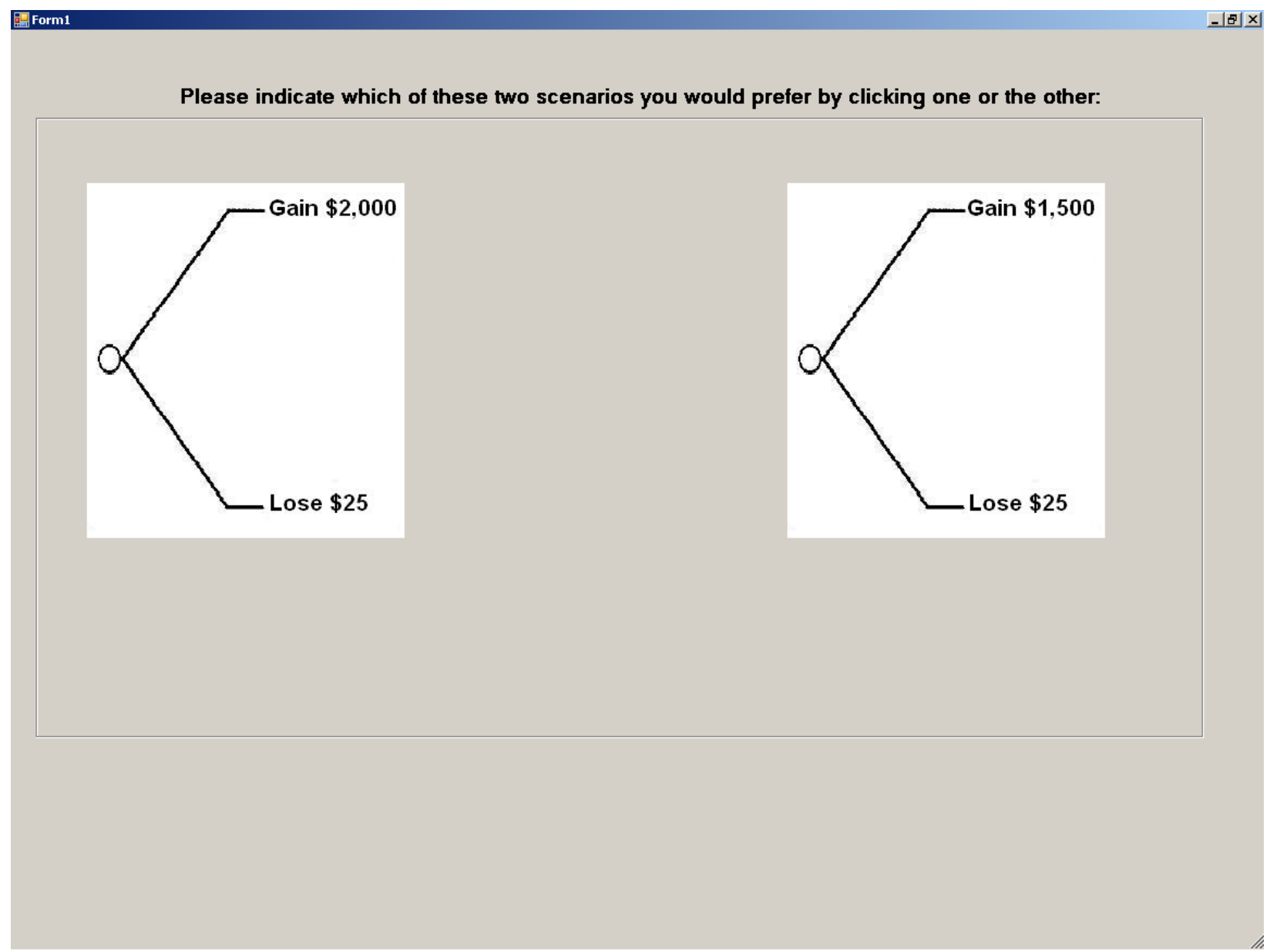




\section{Appendix E}

Subjective Numeracy Scale (SNS)

Page 1 of the SNS, as it appeared to participants:

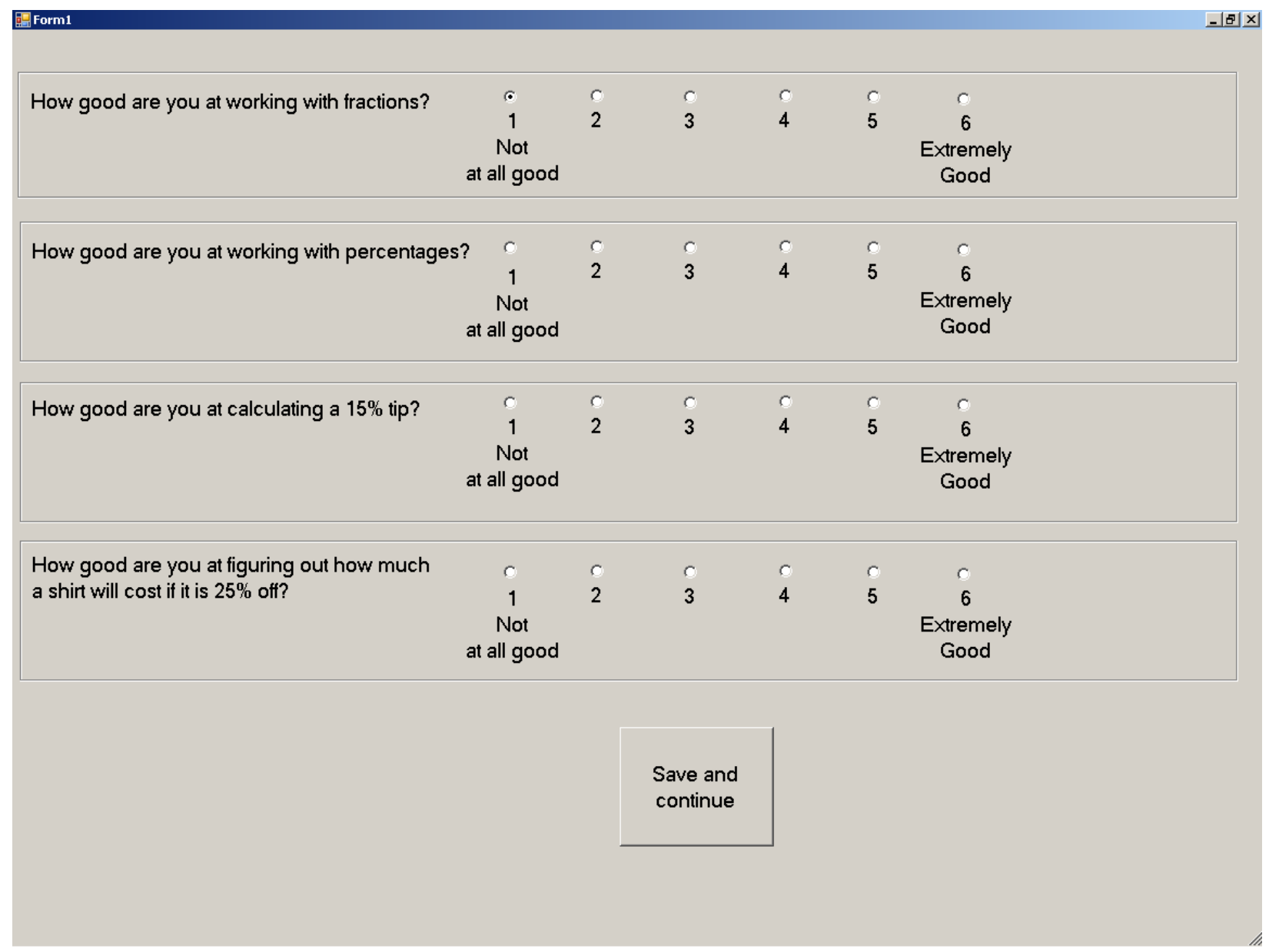


Page 2 of the SNS, as it appeared to participants:

\begin{tabular}{|c|c|c|c|c|c|c|}
\hline SN52 & & & & & & \\
\hline $\begin{array}{l}\text { When reading a newspaper, how helpful } \\
\text { do you find tables and graphs that are parts of a story? }\end{array}$ & $\begin{array}{l}\text { 1 } \\
\text { Not at } \\
\text { all }\end{array}$ & 2 & 3 & 4 & 5 & $\begin{array}{l}c \\
6 \\
\text { Extremely }\end{array}$ \\
\hline $\begin{array}{l}\text { When people tell you the chance of something } \\
\text { happening, do you prefer that they use words } \\
\text { ("it rarely happens") or numbers ("there's a } 1 \% \text { chance")? }\end{array}$ & $\begin{array}{c}{ }_{1} \\
\text { Always } \\
\text { prefer } \\
\text { words }\end{array}$ & 2 & $\frac{c}{3}$ & 4 & 5 & $\begin{array}{c}6 \\
6 \\
\text { Always } \\
\text { prefer } \\
\text { numbers }\end{array}$ \\
\hline $\begin{array}{l}\text { When you hear a weather forecast, do you prefer } \\
\text { predictions using percentages (e.g., "there will be } \\
\text { a } 20 \% \text { chance of rain today") or predictions using } \\
\text { only words (e.g., "there is a small chance of rain today")? }\end{array}$ & $\begin{array}{c}c \\
1 \\
\text { Always } \\
\text { prefer } \\
\text { percentages }\end{array}$ & 2 & 3 & c & 5 & $\begin{array}{c}6 \\
6 \\
\text { Always } \\
\text { prefer } \\
\text { words }\end{array}$ \\
\hline \multirow[t]{2}{*}{ How often do you find numerical information to be useful? } & $\begin{array}{c}c \\
1 \\
\text { Never }\end{array}$ & 2 & 3 & 4 & 5 & $\begin{array}{c}6 \\
6 \\
\text { Very } \\
\text { often }\end{array}$ \\
\hline & Save and Continue & & & & & \\
\hline
\end{tabular}




\section{Appendix F}

Weight Control Subscale of Food Choice Questionnaire (FCQ) and the Health Habits Questionnaire (HHQ)

The Weight Control Subscale of the Food Choice Questionnaire, as it appeared to participants:

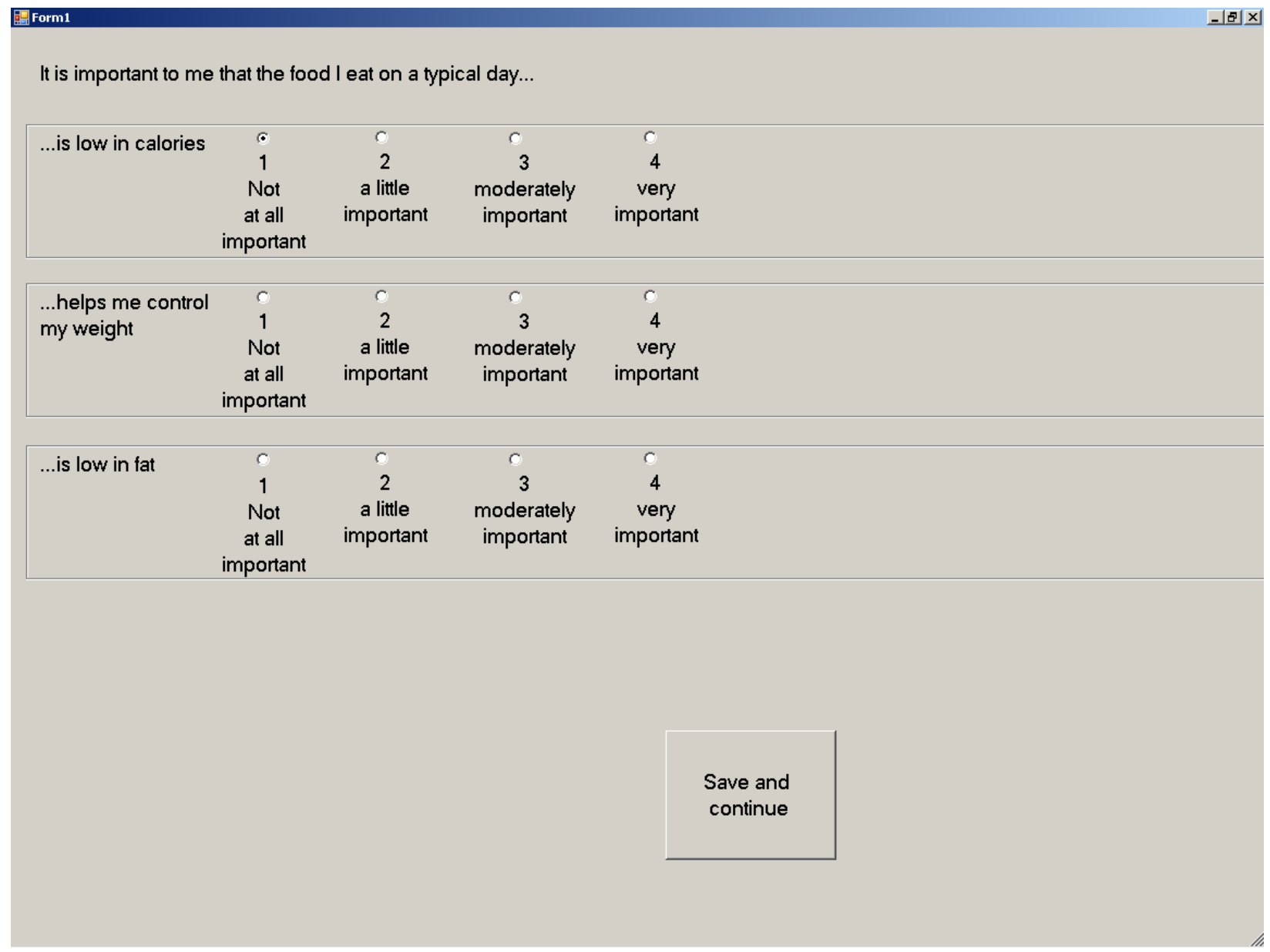


Page 1 of the Health Habits Questionnaire, as it appeared to participants:

\begin{tabular}{|c|c|c|c|c|c|}
\hline \multicolumn{5}{|l|}{ 區WCHH2 } & $-\mid$ 미 $x \mid$ \\
\hline \multicolumn{6}{|l|}{ How frequently do you... } \\
\hline $\begin{array}{l}\text {...check dietary information } \\
\text { on food labels? }\end{array}$ & $\begin{array}{c}\bullet \\
1 \\
\text { Never }\end{array}$ & $\begin{array}{c}\mathrm{C} \\
2 \\
\text { Occasionally }\end{array}$ & $\begin{array}{c}c \\
3 \\
\text { Often }\end{array}$ & $\begin{array}{c}6 \\
4 \\
\text { Almost } \\
\text { always }\end{array}$ & \\
\hline $\begin{array}{l}\text {...count the number of calories } \\
\text { in the food you eat? }\end{array}$ & $\begin{array}{c}c \\
1 \\
\text { Never }\end{array}$ & $\begin{array}{c}\mathrm{c} \\
2 \\
\text { Occasionally }\end{array}$ & $\begin{array}{c}c \\
3 \\
\text { Often }\end{array}$ & $\begin{array}{c}0 \\
4 \\
\text { Almost } \\
\text { always }\end{array}$ & \\
\hline ...put yourself on a diet? & $\begin{array}{c}c \\
1 \\
\text { Never }\end{array}$ & $\frac{\mathrm{c}}{2}$ Occasionally & $\begin{array}{c}c \\
3 \\
\text { Often }\end{array}$ & $\begin{array}{c}6 \\
4 \\
\text { Almost } \\
\text { always }\end{array}$ & \\
\hline ...weigh yourself? & $\begin{array}{c}c \\
1 \\
\text { Never }\end{array}$ & $\begin{array}{c}\mathrm{C} \\
2 \\
\text { Occasionally }\end{array}$ & $\begin{array}{c}c \\
3 \\
\text { Often }\end{array}$ & $\begin{array}{c}c \\
4 \\
\text { Almost } \\
\text { everyday }\end{array}$ & \\
\hline & & & $\begin{array}{l}\text { Save and } \\
\text { continue }\end{array}$ & & \\
\hline
\end{tabular}


Page 2 of the Health Habits Questionnaire as it appeared to participants:

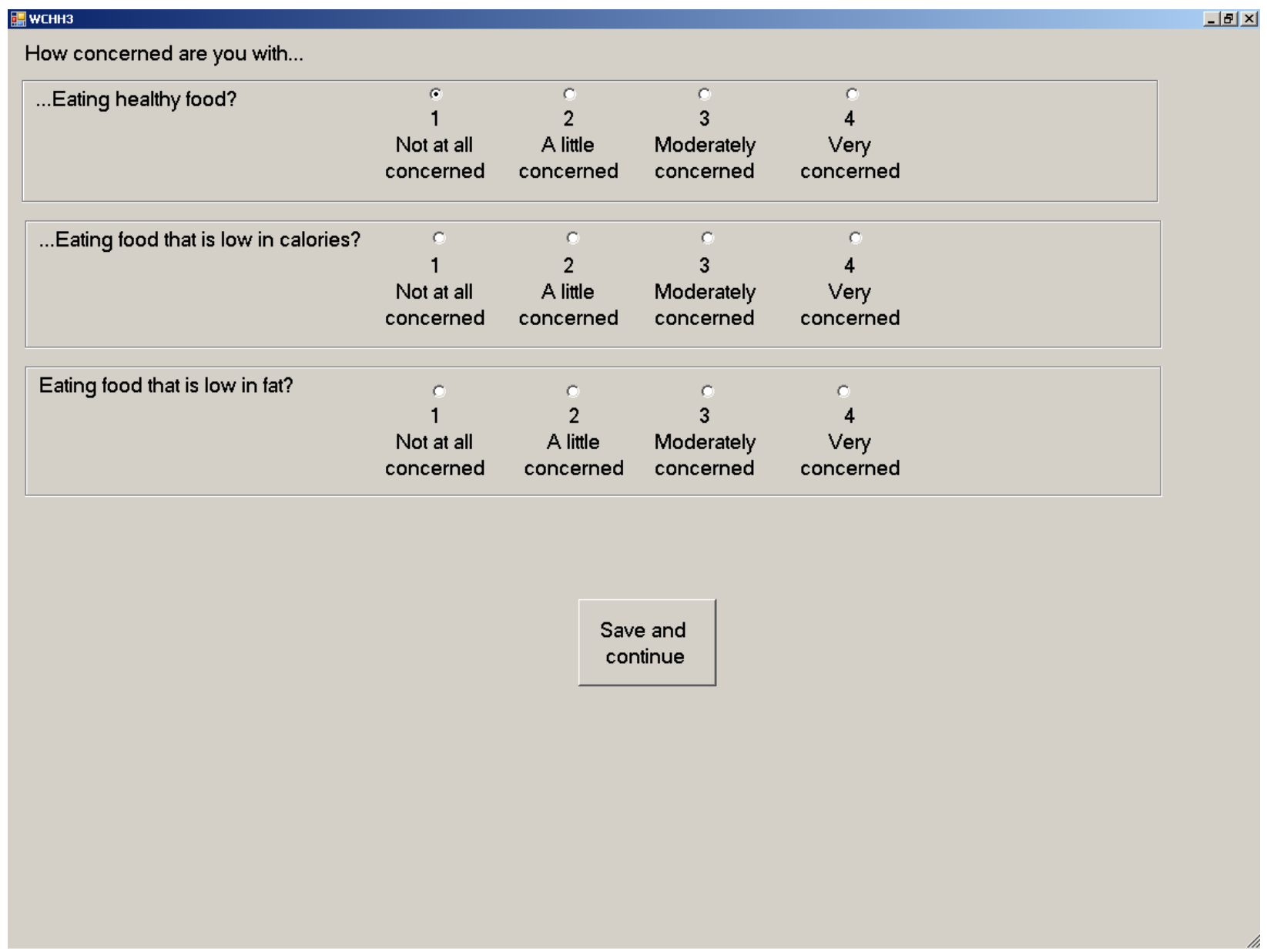


Page 3 of the Health Habits Questionnaire as it appeared to participants:

How concerned are you with..

\begin{tabular}{|c|c|c|c|c|}
\hline $\begin{array}{l}\text {...Checking nutrition } \\
\text { information on food labels? }\end{array}$ & $\begin{array}{c}c \\
1 \\
\text { Not at all } \\
\text { concerned }\end{array}$ & $\begin{array}{c}c \\
2 \\
\text { A little } \\
\text { concerned }\end{array}$ & $\begin{array}{c}\mathrm{C} \\
3 \\
\text { Moderately } \\
\text { concerned }\end{array}$ & $\begin{array}{c}C \\
4 \\
\text { Very } \\
\text { concerned }\end{array}$ \\
\hline $\begin{array}{l}\text {... Eating food that is } \\
\text { low in carbohydrates? }\end{array}$ & $\begin{array}{c}\text { C } \\
1 \\
\text { Not at all } \\
\text { concerned }\end{array}$ & $\begin{array}{c}C \\
2 \\
\text { A little } \\
\text { concerned }\end{array}$ & $\begin{array}{c}\text { C } \\
3 \\
\text { Moderately } \\
\text { concerned }\end{array}$ & $\begin{array}{c}\text { C } \\
4 \\
\text { Very } \\
\text { concerned }\end{array}$ \\
\hline $\begin{array}{l}\text {...Keeping track of the number } \\
\text { of calories in the food you eat? }\end{array}$ & $\begin{array}{c}C \\
1 \\
\text { Not at all } \\
\text { concerned }\end{array}$ & $\begin{array}{c}c \\
2 \\
\text { A little } \\
\text { concerned }\end{array}$ & $\begin{array}{c}\mathrm{C} \\
3 \\
\text { Moderately } \\
\text { concerned }\end{array}$ & $\begin{array}{c}C \\
4 \\
\text { Very } \\
\text { concerned }\end{array}$ \\
\hline
\end{tabular}


Page 4 of the Health Habits Questionnaire as it appeared to participants:

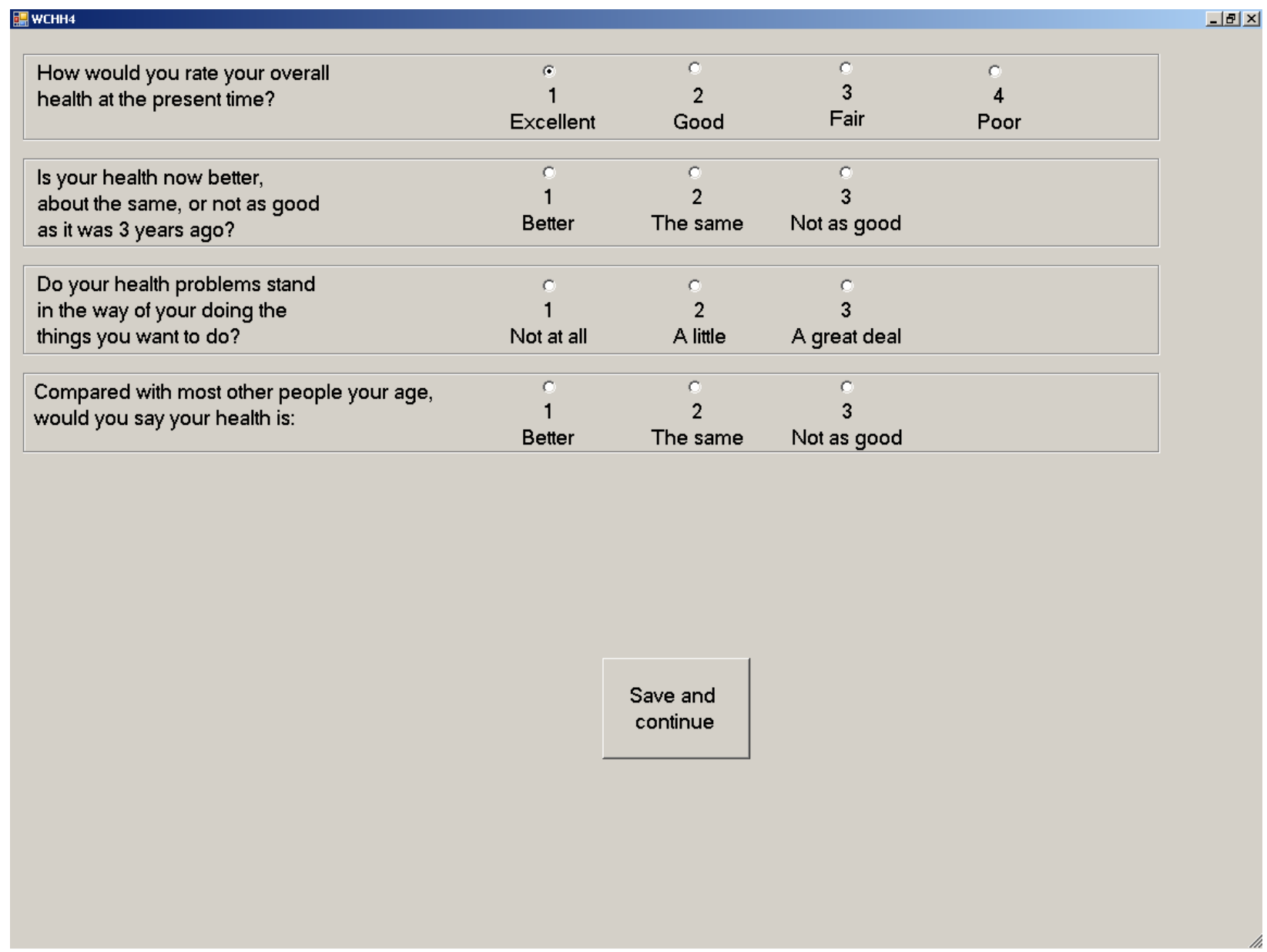


Page 5 of the Health Habits Questionnaire as it appeared to participants:

\begin{tabular}{|c|c|c|c|}
\hline $\begin{array}{l}\text { Compared with most other people your age, } \\
\text { how much do you think you know } \\
\text { about staying healthy? }\end{array}$ & $\begin{array}{c}c \\
1 \\
\text { Not as much }\end{array}$ & $\begin{array}{c}\mathrm{C} \\
2 \\
\text { The same }\end{array}$ & $\begin{array}{c}c \\
3 \\
\text { More }\end{array}$ \\
\hline $\begin{array}{l}\text { Compared with most other people your age, } \\
\text { how much do you think you know } \\
\text { about nutrition? }\end{array}$ & $\begin{array}{c}c \\
1 \\
\text { Not as much }\end{array}$ & $\begin{array}{c}\mathrm{C} \\
2 \\
\text { The same }\end{array}$ & $\begin{array}{c}c \\
3 \\
\text { More }\end{array}$ \\
\hline
\end{tabular}


Page 6 of the Health Habits Questionnaire as it appeared to participants:

Have you ever...

\begin{tabular}{|lcc|}
\hline ...Paid money to & $\odot$ & C \\
join a gym or fitness club? & Yes & No
\end{tabular}

...Paid money for a personl trainer?

Yes No

...Paid money to join a diet program? (e.g. Weight Watchers

or Jenny Craig, or something similar)

Yes $c$

...Paid money for a personal nutritionist?

Yes No

c

$C$

Yes No

..Paid money to purchase home gym equipment?

$\begin{array}{cc}c & c \\ \text { Yes } & \text { No }\end{array}$

Save and continue 


\section{Appendix G}

\section{The Sunk Cost Vignette Questionnaire}

Note: Vignette pairs are grouped together in this appendix for comparison. However, presentation of each vignette within a pair was separated by vignettes that were not a part of that pair.

Vignette Pair 1 (no sunk-cost analogue), as it appeared to participants:

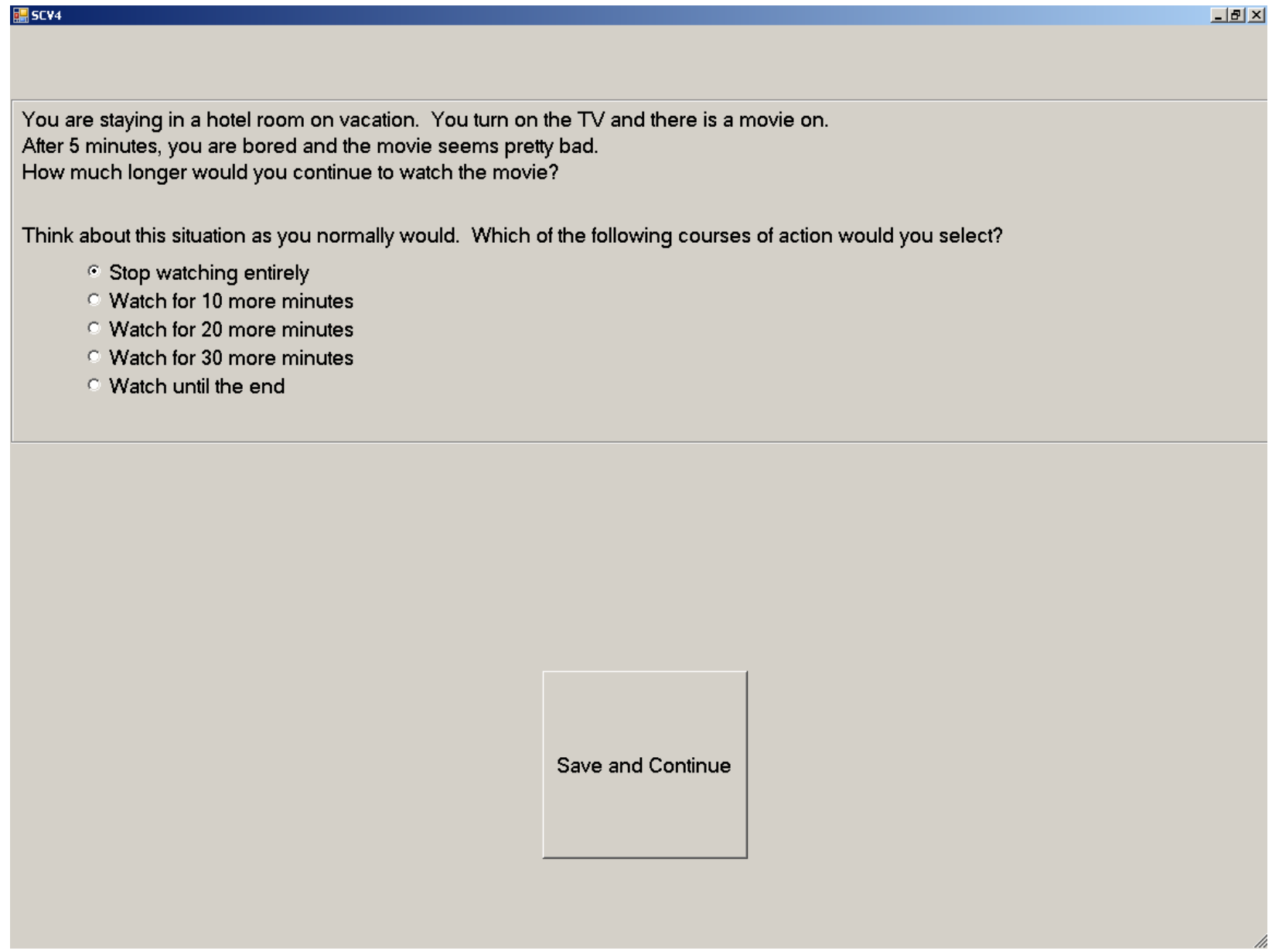


Vignette Pair 1 (sunk-cost analogue), as it appeared to participants:

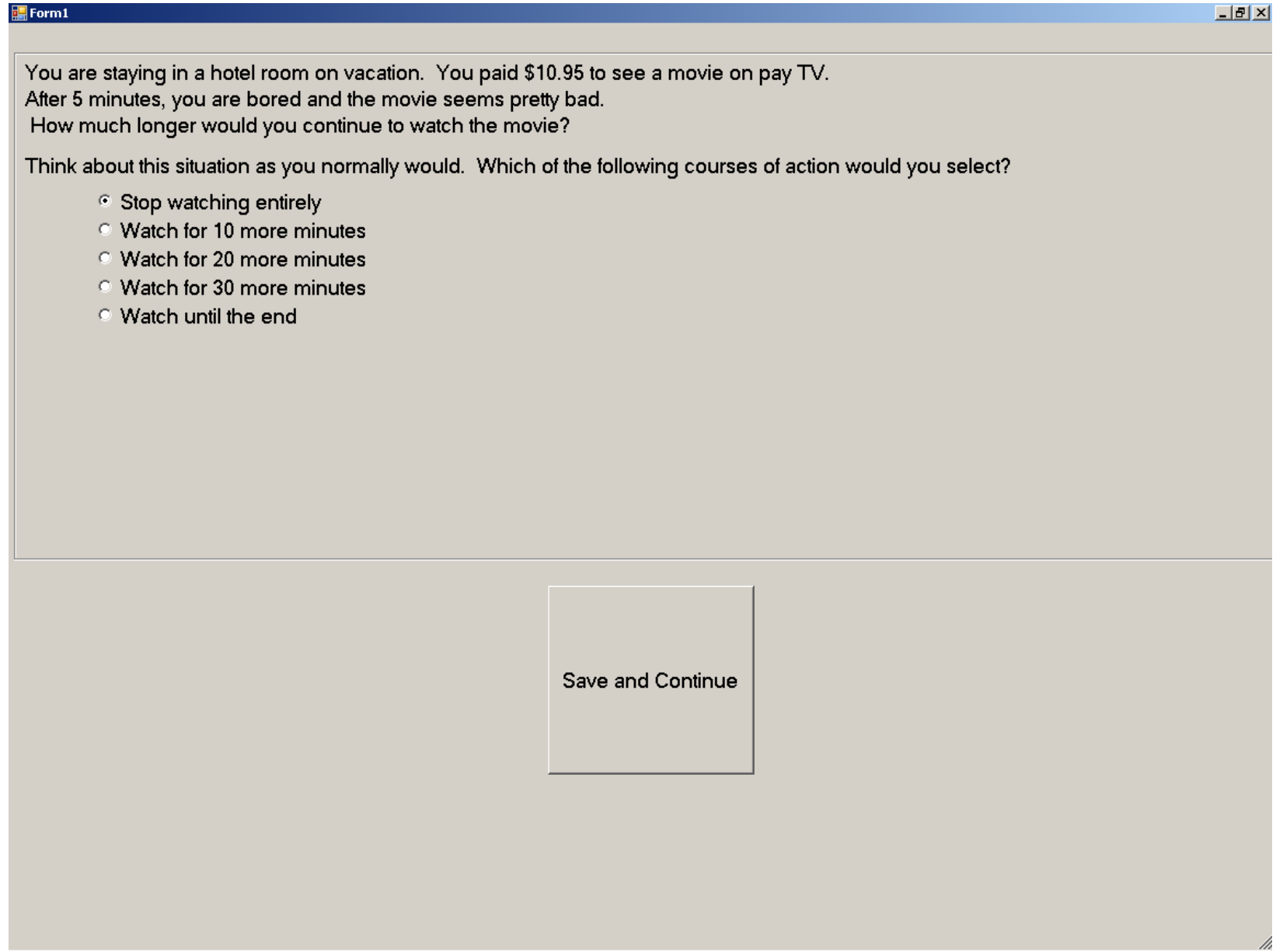


Vignette Pair 2 (no sunk-cost analogue), as it appeared to participants:

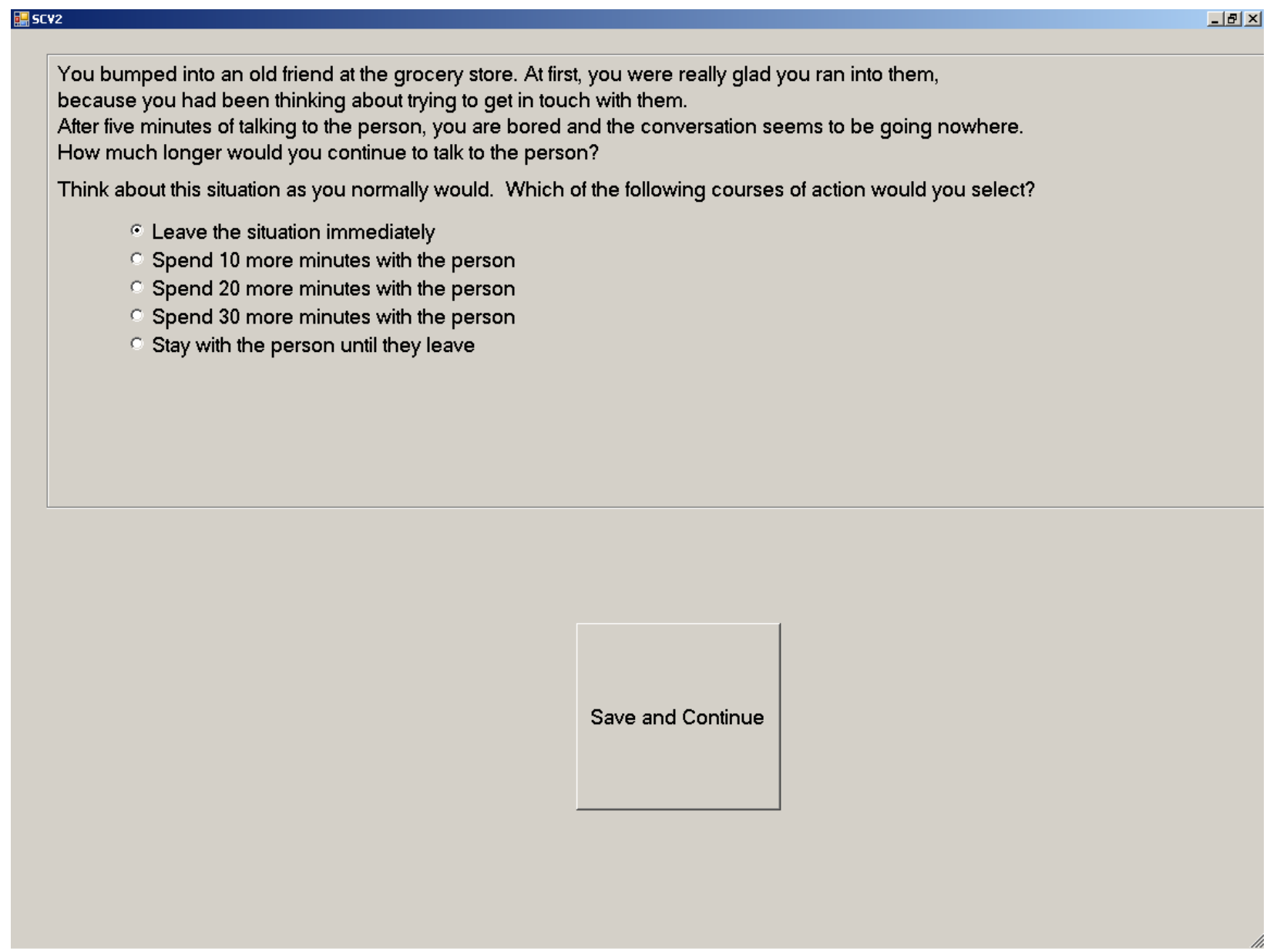


Vignette Pair 2 (sunk-cost analogue), as it appeared to participants:

You paid a tracking service $\$ 10.95$ to find an old friend that you had lost contact with. You arrange to meet the person. At first, it is really great to see them.

After five minutes of talking to the person, you are bored and the conversation seems to be going nowhere.

How much longer would you continue to talk to the person?

Think about this situation as you normally would. Which of the following courses of action would you select?

- Leave the situation immediately

C Spend 10 more minutes with the person

c Spend 20 more minutes with the person

c Spend 30 more minutes with the person

c Stay with the person until they leave 
Vignette Pair 3 (small sunk-cost analogue), as it appeared to participants:

You became friends with a person you met a month ago. Lately, you feel like you don't have much in common with the person, and whenever you are with the person, you are bored and wish that you were doing something else.

Think about this situation as you normally would. Which of the following courses of action would you select?

- Break off the friendship immediately

c Wait for a couple of weeks to see if the friendship improves

C Wait for a month or two to see if the friendship improves

c Wait for 6 months to see if the friendship improves

C Remain committed to the friendship 
Vignette Pair 3 (large sunk-cost analogue), as it appeared to participants:

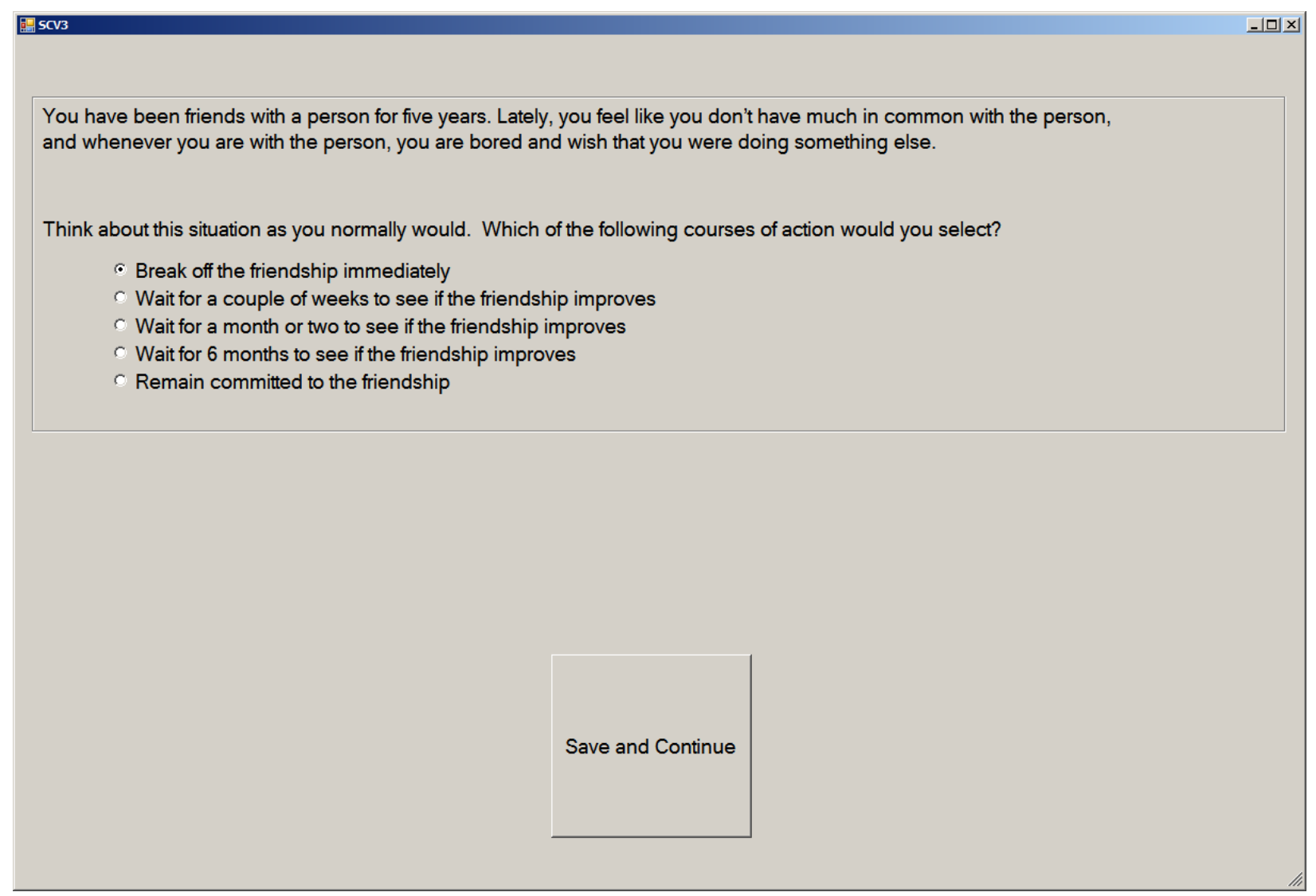


Vignette Pair 4 (small sunk-cost analogue), as it appeared to participants:

You have been working on a project related to one of your hobbies for the past month. Lately, you have lost interest in the project. Whenever you work on the project, you are bored and wish that you were doing something else.

Think about this situation as you normally would. Which of the following courses of action would you select?

- Stop working on the project immediately

c Wait for a couple of weeks to see if interest in the project increases

c Wait for a month or two to see if interest in the project increases

c Wait for six months to see if interest in the project increases

C Remain committed to the project 
Vignette Pair 4 (large sunk-cost analogue), as it appeared to participants:

You have been working on a project related to one of your hobbies for five years. Lately, you have lost interest in the project. Whenever you work on the project, you are bored and wish that you were doing something else.

Think about this situation as you normally would. Which of the following courses of action would you select?

- Stop working on the project immediately

c Wait for a couple of weeks to see if interest in the project increases

c Wait for a month or two to see if interest in the project increases

c Wait for six months to see if interest in the project increases

$\checkmark$ Remain committed to the project

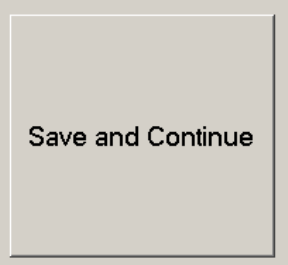




\section{Appendix $\mathrm{H}$}

\section{Spendthrift-Tightwad Scale (STS)}

STS page 1, as it appeared to participants

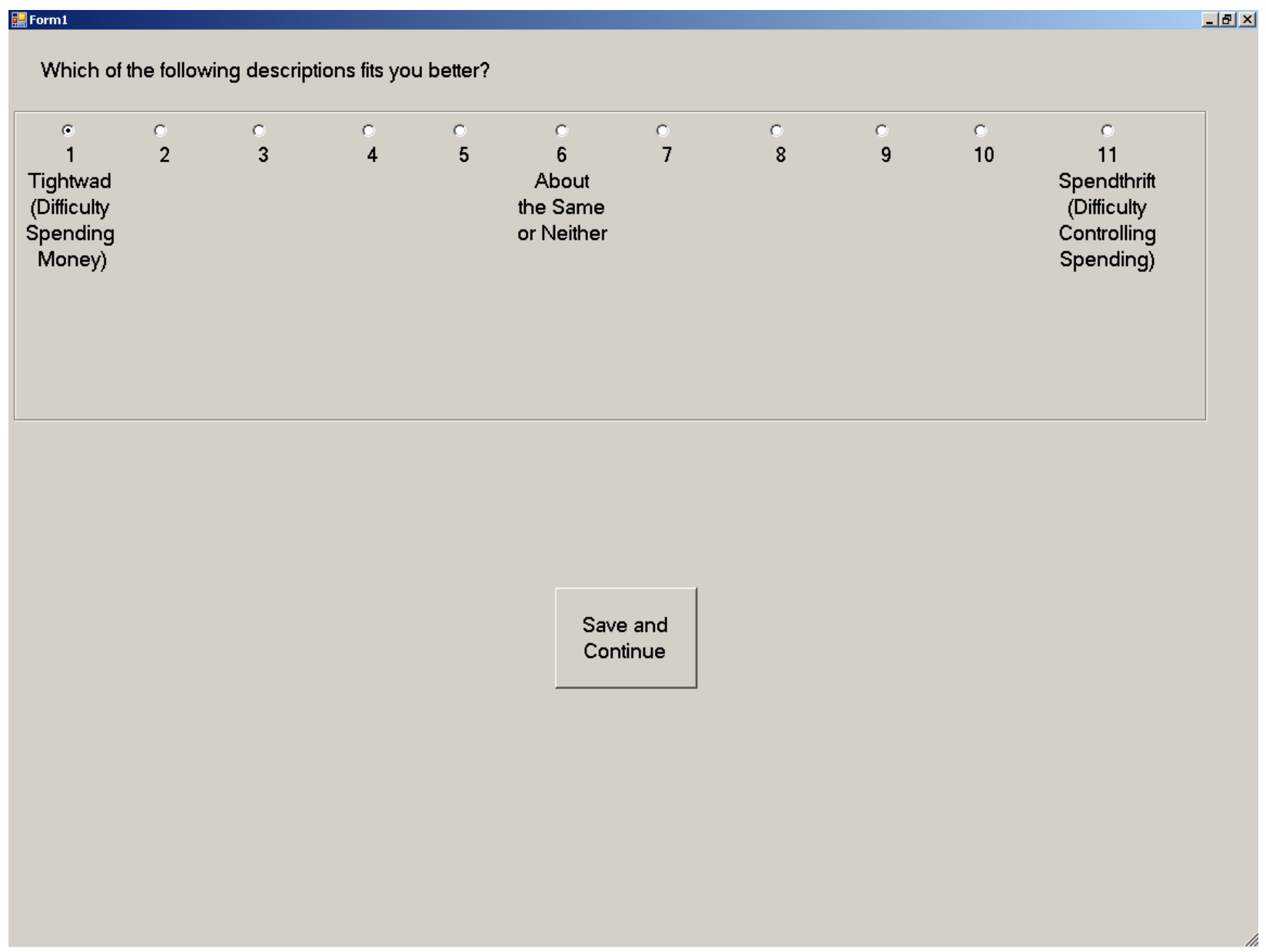


STS page 2, as it appeared to participants

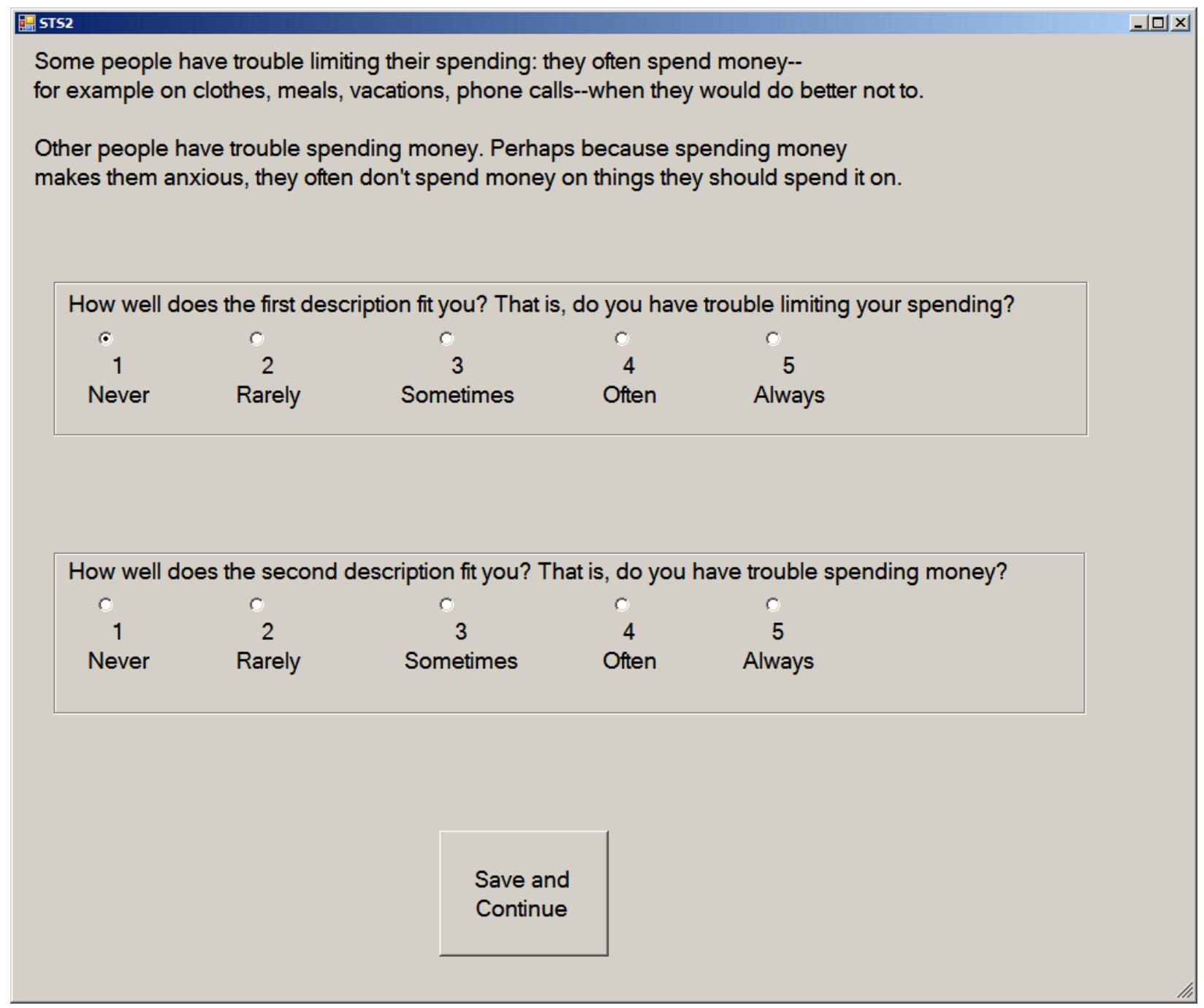


STS page 3, as it appeared to participants

阳 STS3

Following is a scenario describing the behavior of two shoppers. After reading about each shopper, please answer the question that follows.

Mr. A is accompanying a good friend who is on a shopping spree at a local mall. When they enter a large department store, Mr. A sees that the store has a "One-day-only-sale" where everything is priced $10-60 \%$ off. He realizes he doesn't need anything, yet can't resist and ends up spending almost $\$ 100$ on stuff.

Mr. B is accompanying a good friend who is on a shopping spree at a local mall. When they enter a large department store, Mr. B sees that the store has a "One-day-only-sale" where everything is priced $10-60 \%$ off. He figures he can get great deals on many items that he needs, yet the thought of spending the money keeps him from buying the stuff.

In terms of your own behavior, who are you more similar to, Mr. A or Mr. B?

1

Mr. A
2

About the same

or neither
4

Mr. B 


\section{Appendix I}

Future Time Perspective (FTP) Scale

\section{FTP Scale page 1, as it appeared to participants}

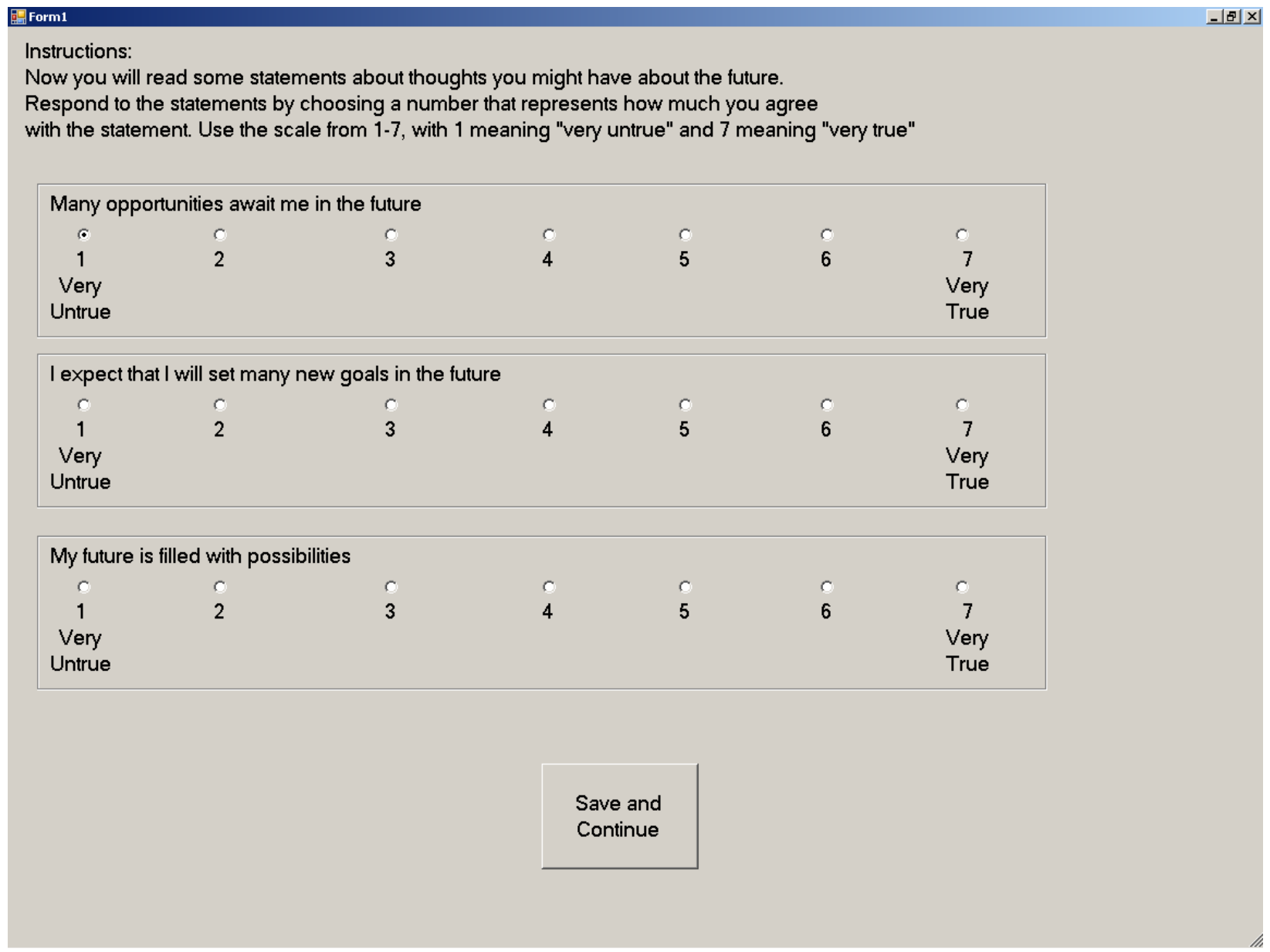


FTP Scale page 2, as it appeared to participants

\begin{tabular}{|c|c|c|c|c|c|c|c|}
\hline 匵FTP2 & & & & & & & $-\mid[|a| x \mid$ \\
\hline \multicolumn{8}{|c|}{ Most of my life lies ahead of me } \\
\hline • & c & c & $c$ & $c$ & c & $c$ & \\
\hline 1 & 2 & 3 & 4 & 5 & 6 & 7 & \\
\hline Very & & & & & & Very & \\
\hline Untrue & & & & & & True & \\
\hline \multicolumn{8}{|c|}{ My future seems infinite to me } \\
\hline$c$ & c & c & c & $c$ & c & $c$ & \\
\hline 1 & 2 & 3 & 4 & 5 & 6 & 7 & \\
\hline Very & & & & & & Very & \\
\hline Untrue & & & & & & True & \\
\hline \multicolumn{8}{|c|}{ I could do anything I want in the future } \\
\hline$c$ & $c$ & $c$ & c & $c$ & c & c & \\
\hline \multirow{4}{*}{$\begin{array}{c}1 \\
\text { Very } \\
\text { Untrue }\end{array}$} & 2 & 3 & 4 & 5 & 6 & 7 & \\
\hline & & & & & & Very & \\
\hline & & & & & & True & \\
\hline & & & & $\begin{array}{l}\text { Save and } \\
\text { Continue }\end{array}$ & & & \\
\hline & & & & & & & \\
\hline & & & & & & & \\
\hline
\end{tabular}


FTP Scale page 3, as it appeared to participants

\begin{tabular}{|c|c|c|c|c|c|c|c|}
\hline 嘼FP3 & & & & & & & $-|[\square] x|$ \\
\hline There $\mathrm{i}$ & f tin & nak & & & & & \\
\hline • & $c$ & $c$ & c & $c$ & $c$ & c & \\
\hline 1 & 2 & 3 & 4 & 5 & 6 & 7 & \\
\hline Very & & & & & & Very & \\
\hline Untrue & & & & & & True & \\
\hline I have & lat $\mathrm{t}$ & & & & & & \\
\hline$c$ & $c$ & $c$ & $c$ & $c$ & $c$ & $c$ & \\
\hline 1 & 2 & 3 & 4 & 5 & 6 & 7 & \\
\hline Very & & & & & & Very & \\
\hline Untrue & & & & & & True & \\
\hline There & mite & $\mathrm{m}$ & & & & & \\
\hline$c$ & $c$ & $c$ & $c$ & $c$ & $c$ & $c$ & \\
\hline 1 & 2 & 3 & 4 & 5 & 6 & 7 & \\
\hline Very & & & & & & Very & \\
\hline Untrue & & & & & & True & \\
\hline As I ge & egi & tim & & & & & \\
\hline$c$ & c & $c$ & c & $c$ & $c$ & $c$ & \\
\hline 1 & 2 & 3 & 4 & 5 & 6 & 7 & \\
\hline Very & & & & & & Very & \\
\hline Untrue & & & & & & True & \\
\hline & & & & $\begin{array}{l}\text { Save and } \\
\text { Continue }\end{array}$ & & & \\
\hline
\end{tabular}




\section{Appendix $\mathbf{J}$}

\section{Demographics Form}

Page 1 of the demographics form as it appeared to participants

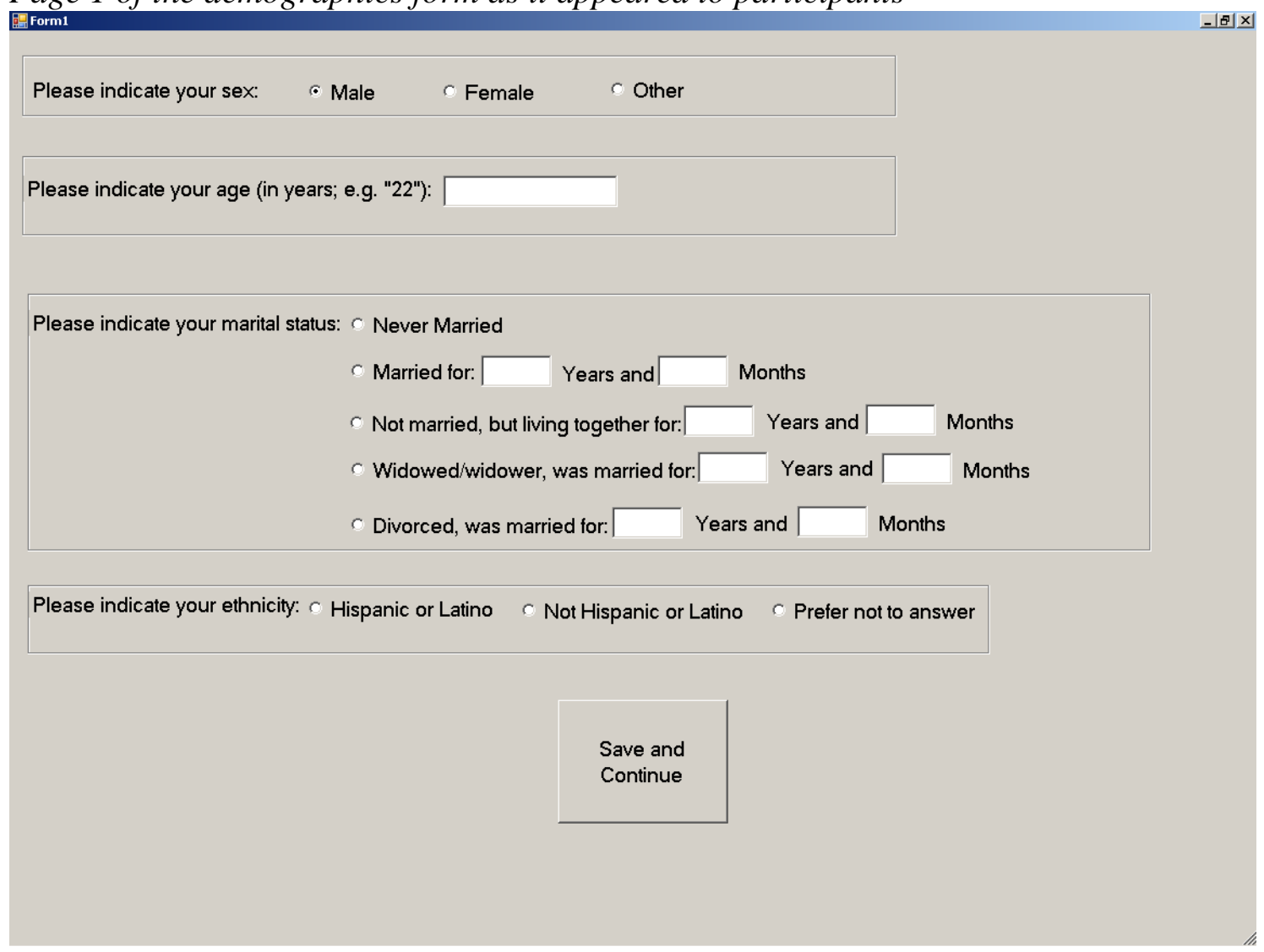


Page 2 of the demographics form as it appeared to participants

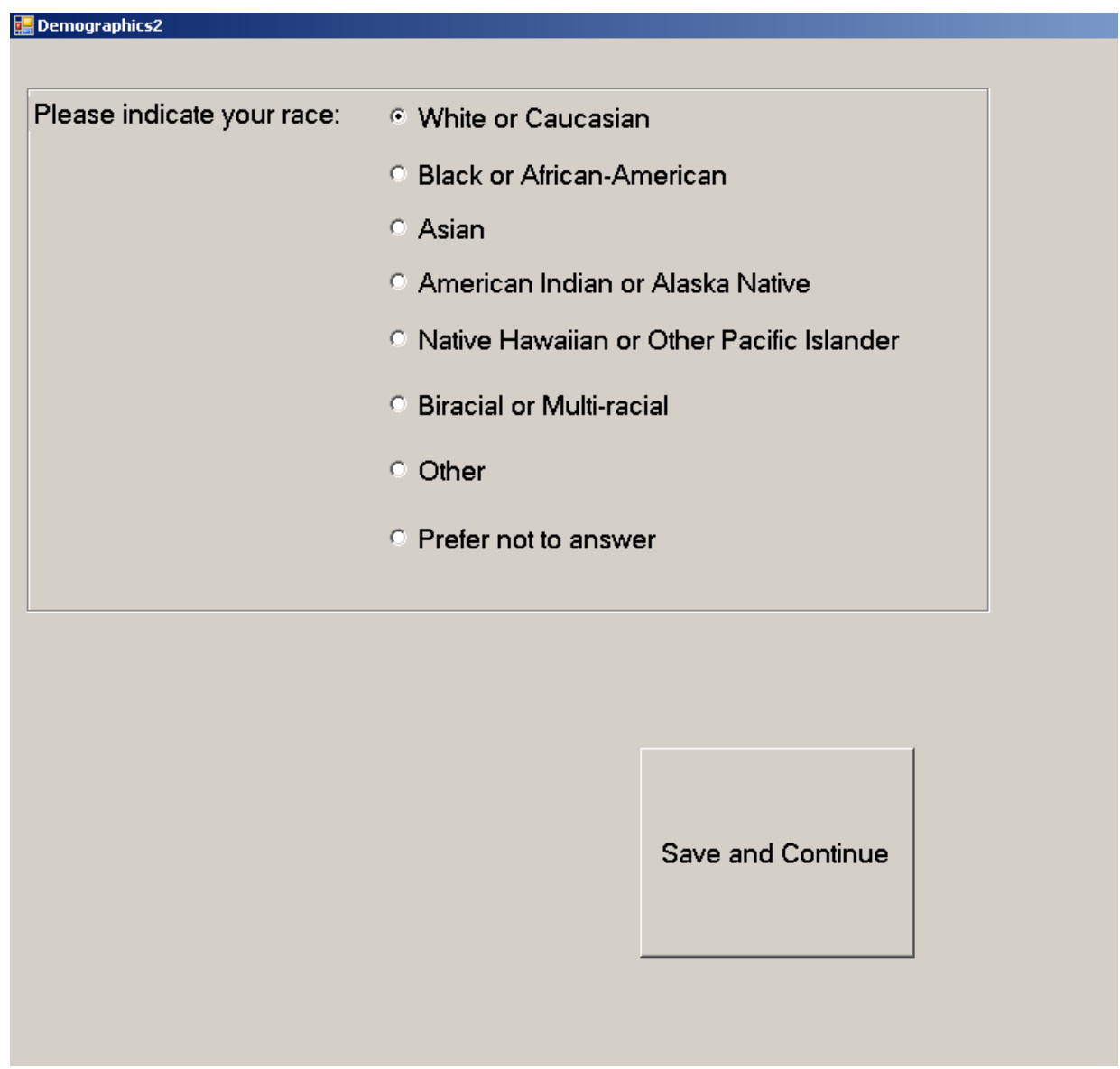


Page 3 of the demographics form as it appeared to participants

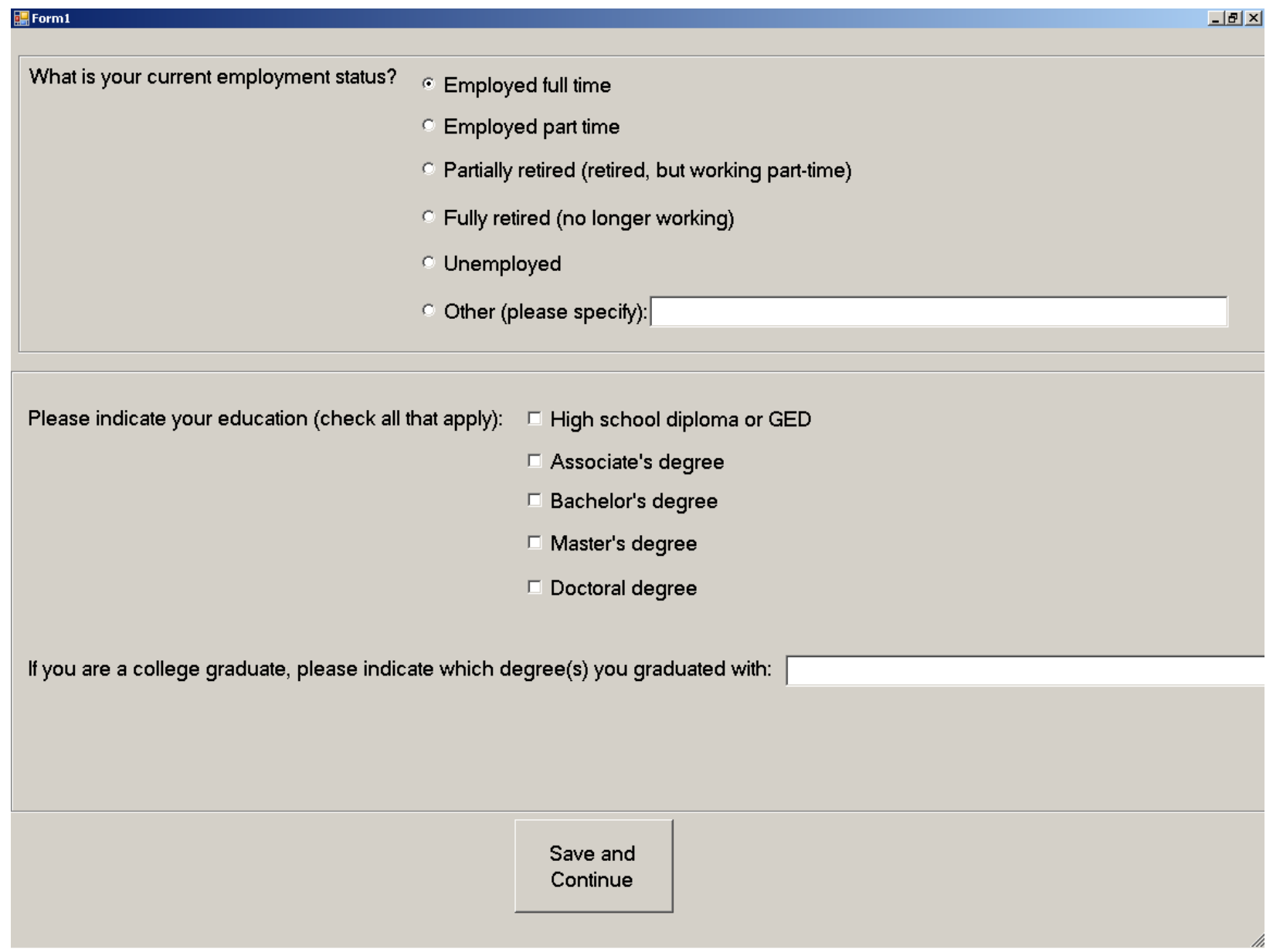


Page 4 of the demographics form as it appeared to participants

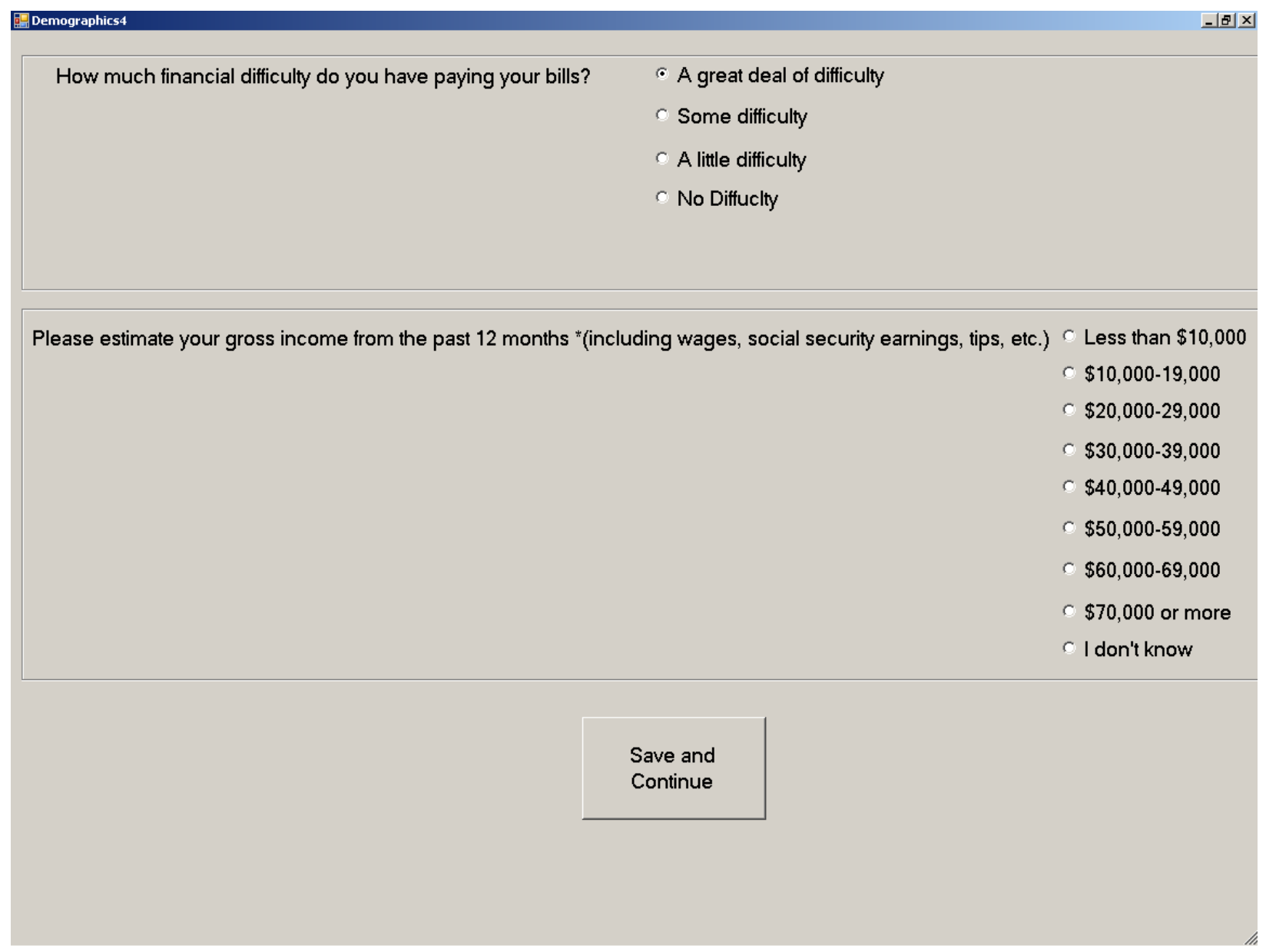


Page 5, of the demographics form, which appeared only for younger adults

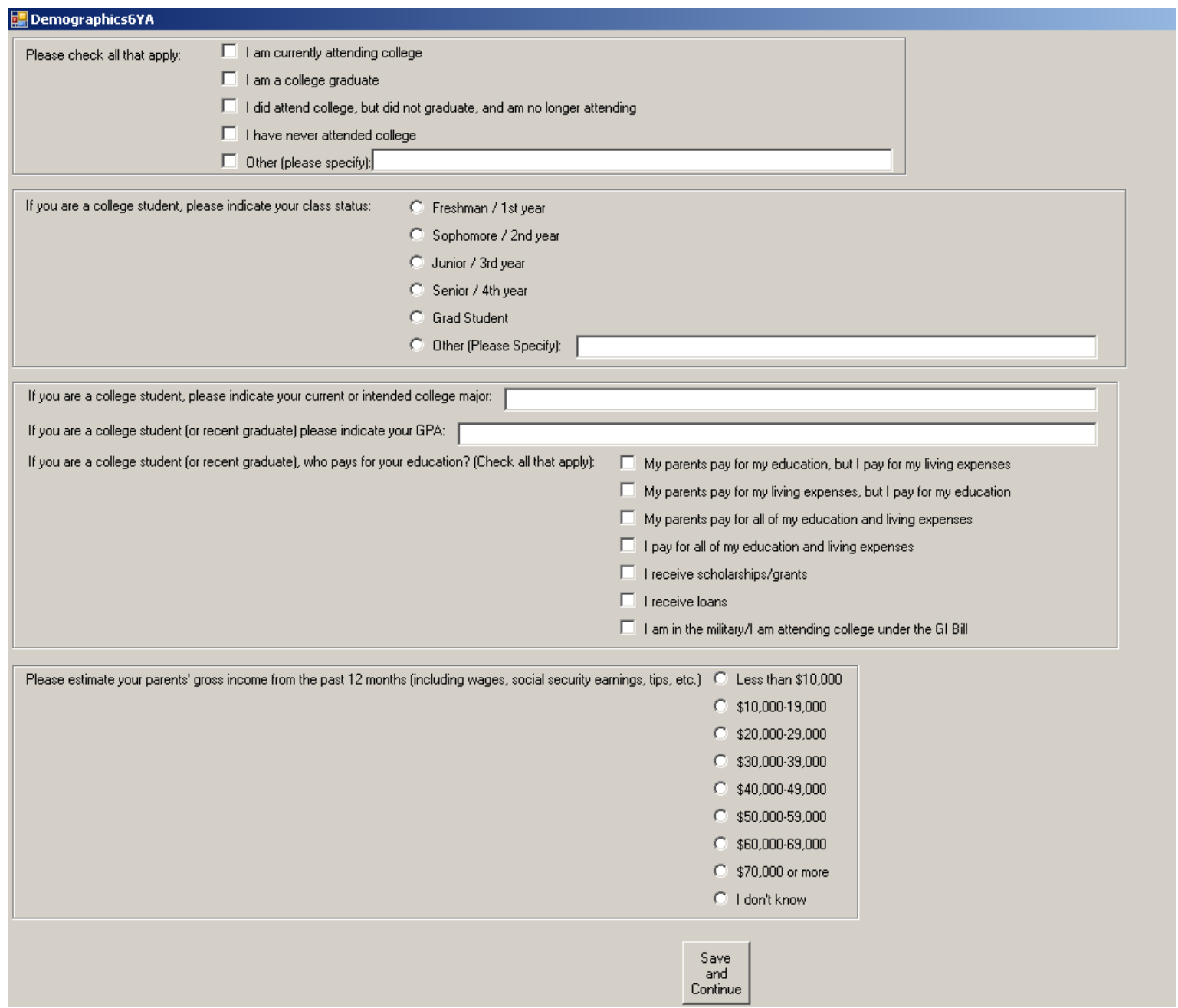


Page 5 of the demographics form, which appeared only for older adults

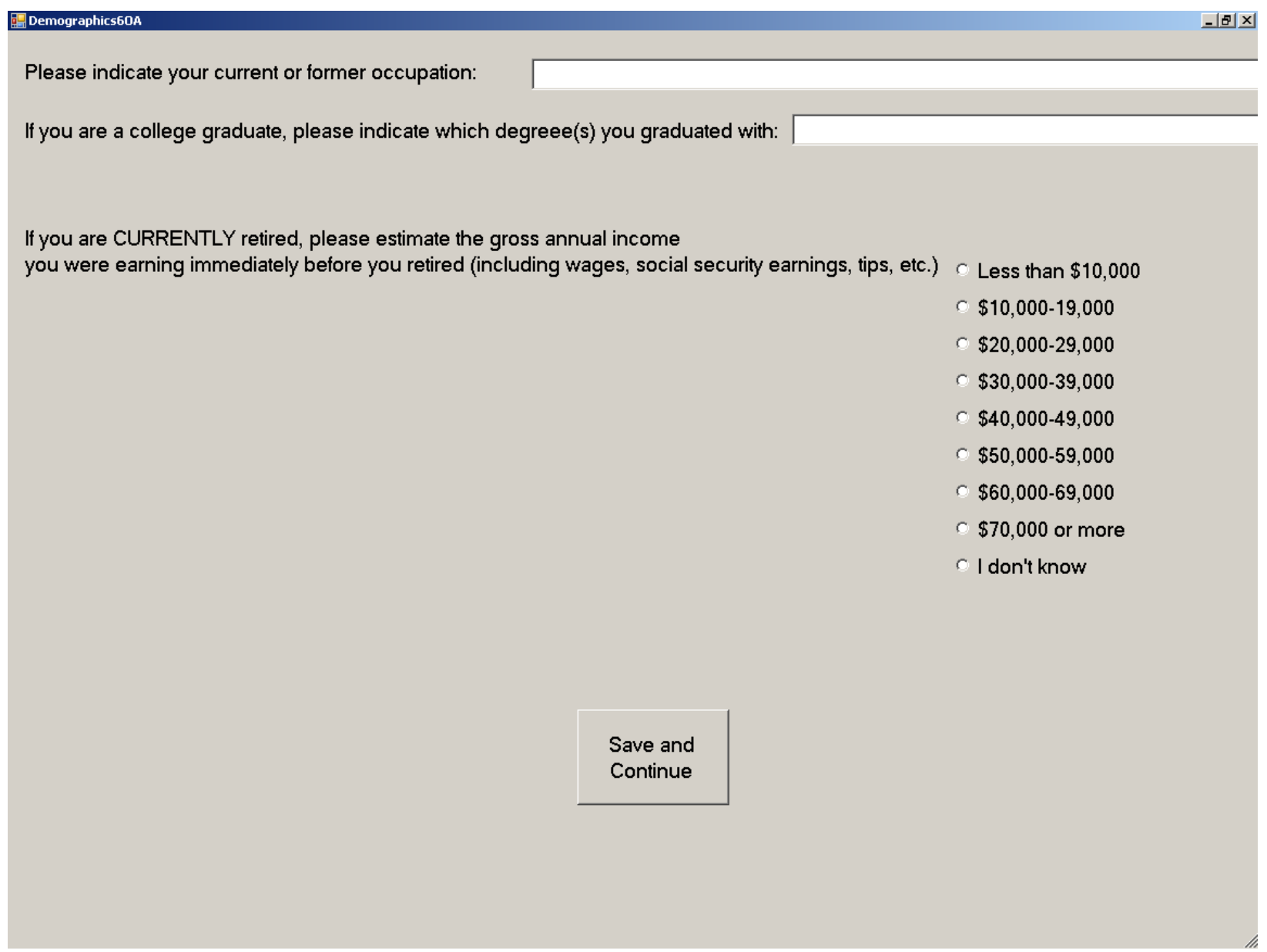




\section{Appendix K}

Consent Form

This study is being conducted by Leo Schlosnagle, M.S. under the supervision of JoNell Strough, Ph.D. For more information about this research you can contact Leo Schlosnagle at (240) 925-7079 or Dr. JoNell Strough at (304) 293-2001 x31648.

If you choose to participate in this study, you will be asked a series of questions about yourself and how you think about food. Any information about you that is obtained as a result of your participation in this research will be kept as confidential as legally possible. Your research records, just like hospital records, may be subpoenaed by court order or may be inspected by federal regulatory authorities without your additional consent. In any publications that result from this research, neither your name nor any information from which you might be identified will be published without your consent.

There are no known or expected risks from participating in this study, except for the mild frustration associated with answering the questions. Your participation in this study is voluntary. You may choose to not participate in this study, or you may choose to withdraw your consent and stop participating in this study at any point after the study begins.

If you are a student, refusal to participate or withdrawal from this study will not affect your class standing or grades and will involve no penalty to you. You have been given the opportunity to ask questions about the research, and you have received answers concerning areas you did not understand.

Printed Name

Signature Date 